\title{
Image-guided and adaptive radiation therapy with 3D ultrasound imaging
}

Citation for published version (APA):

van der Meer, S. (2015). Image-guided and adaptive radiation therapy with 3D ultrasound imaging. [Doctoral Thesis, Maastricht University]. Datawyse / Universitaire Pers Maastricht.

https://doi.org/10.26481/dis.20151202sm

Document status and date:

Published: 01/01/2015

DOI:

10.26481/dis.20151202sm

Document Version:

Publisher's PDF, also known as Version of record

\section{Please check the document version of this publication:}

- A submitted manuscript is the version of the article upon submission and before peer-review. There can be important differences between the submitted version and the official published version of record.

People interested in the research are advised to contact the author for the final version of the publication, or visit the DOI to the publisher's website.

- The final author version and the galley proof are versions of the publication after peer review.

- The final published version features the final layout of the paper including the volume, issue and page numbers.

Link to publication

\footnotetext{
General rights rights.

- You may freely distribute the URL identifying the publication in the public portal. please follow below link for the End User Agreement:

www.umlib.nl/taverne-license

Take down policy

If you believe that this document breaches copyright please contact us at:

repository@maastrichtuniversity.nl

providing details and we will investigate your claim.
}

Copyright and moral rights for the publications made accessible in the public portal are retained by the authors and/or other copyright owners and it is a condition of accessing publications that users recognise and abide by the legal requirements associated with these

- Users may download and print one copy of any publication from the public portal for the purpose of private study or research.

- You may not further distribute the material or use it for any profit-making activity or commercial gain

If the publication is distributed under the terms of Article $25 \mathrm{fa}$ of the Dutch Copyright Act, indicated by the "Taverne" license above, 


\section{Production}

Cover: $\quad$ Skadi van der Meer i.s.m. Datawyse

Printing: Datawyse | Universitaire Pers Maastricht

ISBN: $\quad 9789461595027$

\section{Copyrights}

(C) Copyright S. van der Meer, Maastricht 2015

Chapter 2:Physics in Medicine \& Biology, Institute of Physics and IOP Publishing 2015 Chapter 3:Technology in Cancer Research \& Treatment, SAGE Publications 2015 Chapter 4:Medical Physics, American Association of Physicists in Medicine 2013 Chapter 5:Int. Journal of Radiation Oncology* Biology* Physics, Elsevier Inc, 2013

No parts of this publication may be reproduced in any form without prior written permission of the holder of the copyrights.

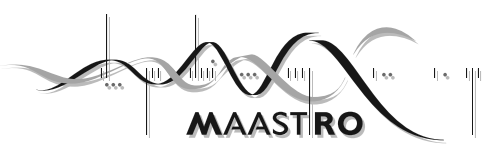

CLINIC 


\title{
Image-Guided and Adaptive Radiation Therapy with 3D Ultrasound Imaging
}

\author{
Proefschrift \\ ter verkrijging van de graad van doctor aan de Universiteit Maastricht, \\ op gezag van de Rector Magnificus prof. dr. L.L.G. Soete, \\ volgens het besluit van het College van Decanen, \\ in het openbaar te verdedigen \\ op woensdag 2 december 2015 om 12.00 uur \\ door \\ Skadi van der Meer
}

$\underbrace{\text { UNIVERSITAIRE }}$ 


\section{Promotor}

Prof. dr. ir. F. Verhaegen

\section{Copromotor}

Dr. L.C.H.W. Lutgens

\section{Beoordelingscommissie}

Prof. dr. ir. W.H. Backes (Voorzitter)

Dr. M.E. Kooi

Prof. dr. ir. J.J.W. Lagendijk, Universitair Medisch Centrum Utrecht

Prof. dr. med. F. Lohr, Universitätsmedizin Mannheim 


\section{Contents}

\section{Introduction}

Chapter 1 Introduction and outline of the thesis 9

Chapter 2 Review of ultrasound image guidance in external beam radiotherapy: 15 I. Treatment planning and inter-fraction motion management

\section{System Robustness}

Chapter 3 Consequences of intermodality registration errors for intramodality 55 3D ultrasound IGRT

\section{Clinical Studies}

Chapter 4 Critical assessment of intramodality 3D ultrasound imaging for prostate IGRT compared to fiducial markers

Chapter 5 Active Breathing Control in Combination With Ultrasound Imaging: A Feasibility Study of Image Guidance in Stereotactic Body Radiation Therapy of Liver Lesions

\section{Towards Adaptive Radiotherapy}

Chapter 6 Simulation of pseudo-CT images based on deformable image registration of ultrasound images: a proof of concept for transabdominal ultrasound imaging of the prostate during radiotherapy

\section{Discussion}

Chapter 7 Summary, general discussion and future perspectives

List of publications

Dankwoord

Curriculum vitae 

INTRODUCTION 



\section{CHAPTER}

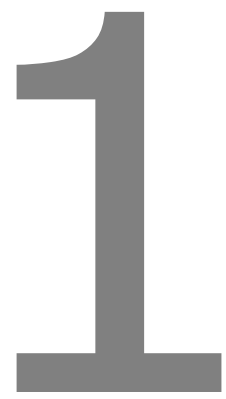

Introduction and outline of the thesis 



\section{INTRODUCTION}

\section{Radiotherapy treatment}

Together with surgery and chemotherapy radiotherapy is one of the three main modalities for treating cancer. Radiotherapy can be administered from outside the body (external) as well as from the inside by positioning radioactive sources inside the patient (brachytherapy), but in this thesis we are only reporting on external beam radiotherapy.

The main principle in radiotherapy is to destroy the tumor using ionizing radiation beams, while causing a minimum amount of damage to the healthy tissue. In particular sparing possible nearby organs at risk (OAR) with a low dose tolerance is very important. Therefore the tumor region to be irradiated has to be localized relative to the patient anatomy; a treatment plan is designed applying anatomical information based on a snapshot in time, i.e. the planning computed tomography (CT)-scan, typically made one week before the start of the actual treatment. A radiotherapy treatment can consist of a single irradiation (fraction) but more often, the patient is receiving the prescribed dose in multiple fractions. The number of fractions depends on the type of cancer that is being treated and its surrounding tissue. A typical radiotherapy treatment involves daily irradiation for several weeks. This is done to allow the healthy tissue to recover between the various fractions of treatment. Because of this fractionated schedule, it is important to maintain the same geometric and dosimetric accuracy across all treatment sessions. Geometric uncertainties like organ motion and patient set-up during treatment are important sources of error in radiotherapy. In order to avoid inadequate coverage of the tumor, margins of a few millimeters are added to the irradiated volume to account for these geometric uncertainties ${ }^{1-4}$. In particular accurate margins are required for treatment plans with tight dose distributions around the target volume like in Intensity-Modulated Radiation Therapy (IMRT) ${ }^{5,6}$, but traditionally, larger set-up margins are used to correct for organ motion. Theoretically, the more accurate the patient positioning system, the more the margins can be reduced. During treatment it is therefore crucial to accurately reproduce the position of the patient when the plan was prepared.

\section{Image Guided Radiotherapy}

It is important to determine the position of the treatment target prior to each radiotherapy treatment. Different imaging systems have been used to determine the position of the region of interest (ROI), some can also be used to estimate the volume and shape of the $\mathrm{ROI}$, in an approach referred to as image guided radiation therapy (IGRT). Among these systems, $x$-ray imaging, currently with electronic portal imaging (EPI) ${ }^{7}$ devices and in the past with radiographic film ${ }^{8,9}$, are well established techniques. At the treatment stage, bony structures are localized and, if their position is different from their simulated planning position, a correction is applied to the patient's position. Nowadays most linear accelerators are equipped with cone beam CT (CBCT) enabling online three-dimensional (3D) volumetric CT-like images of the patient at treatment stage ${ }^{10,11}$. But both EPI and $\mathrm{CBCT}$ rely on $\mathrm{X}$-ray attenuation which inherently limits soft-tissue contrast. Therefore, the visibility of the tumor is still limited and positioning is mostly done on the basis of the 
bony anatomy. Soft tissue targets such as the prostate may move with respect to the bones, and standard x-ray imaging may not offer sufficient contrast to image such targets directly. Therefore other techniques have been introduced to deal with this problem, e.g. fiducial markers (FMs) implanted in tissue ${ }^{12}$, electromagnetic beacons ${ }^{13}, \mathrm{MRI}^{14,15}$ and US imaging $^{16-19}$.

Besides imaging of the target, imaging of the tumor surroundings is desirable for a variety of reasons. One of them is that daily images of the tumor and surroundings allow the reconstruction of the accumulated dose to the tumor and OARs. This capability will provide more insight in the dose effect relationship for various tissues.

\section{US Guided Radiotherapy}

Among the available IGRT techniques there is a growing interest in US imaging. It offers an attractive alternative for imaging radiotherapy targets outside the skull and lung. US imaging systems offer nonionizing, real-time volumetric imaging with excellent soft-tissue contrast and is commonly used for image guidance in brachytherapy (robotic ${ }^{20}$ and nonrobotic ${ }^{21}$ ), biopsy ${ }^{22}$, and other medical procedures. Rapidly developing two- , three- and four-dimensional (2D, 3D and 4D) anatomical and functional imaging capabilities for imaging in-between (inter-fraction) and during (intra-fraction) enable a range of possibilities in the radiotherapy field. In Chapter 2 an extensive review is given about US IGRT.

\section{OBJECTIVE OF THE STUDY}

The general objective of this study is to determine the accuracy of a 3D US imaging technique for image guidance, to determine the daily position and volume of prostate and liver lesions and analyze whether the accuracy of 3D US is compatible with the accuracy of EPI for image guidance. The final goal is to have a non-invasive and accurate technique for image guidance with the feasibility to correct for inter-fraction organ motion.

The first step in this study is to determine the accuracy and the reproducibility of US imaging for image guidance. The effect of probe pressure on target position will be determined. The inter- and intra-operator variation will be investigated for two groups of patients (prostate and liver). We will, however, aim to go beyond geometrical treatment correction. We will also propose a concept to determine the true delivered dose as compared to the planned dose with the use of US imaging. Knowledge about the true delivered dose is crucial to aid decisions to modify the treatment during its course ${ }^{23,24}$.

\section{OUTLINE OF THE THESIS}

In this Chapter 1, a general introduction is given on the background and importance of image guided radiotherapy, with a focus on the use of ultrasound. A more elaborate overview on the use of ultrasound guided radiotherapy is presented in Chapter 2, where a review of published methods is given. The role of US imaging for treatment planning is 
described, followed by a section on its use for monitoring inter-fraction motion. The current applications of US to IGRT for various cancer sites are discussed, and the review concludes with a brief outlook towards the future of US applications in RT.

In Chapter 3, the error propagation in daily patient positioning due to potential registration errors at the planning stage was assessed. With simple examples, we tried to give a better understanding of the system and prevent errors due to simple mistakes or misinterpretations.

The accuracy of clinical US IGRT implementation for prostate and liver are discussed in the following two chapters. In Chapter $\mathbf{4}$ we determined the influence of various factors involved in the workflow of US scanning for prostate cancer patients. We studied the inter- and intra-operator variability of 3DUS intramodality matching, and the influence of inter-operator probe pressure on the prostate position while using a strict bladder filling protocol. The 3DUS prostate localization was compared to MV EPI localization based on FMs in a patient study. In Chapter 5 we investigated the use of US IGRT application in liver treatments. The goal is to establish the accuracy, with in particular the user variability of 3DUS imaging with and without active breathing control (ABC) for IGRT of liver lesions.

Chapter 6 focuses on the possible extra opportunities of the daily US imaging besides IGRT. Besides for position verification, imaging of patient anatomy during treatment is a necessity for adaptive radiotherapy and can be used to perform daily dose recalculation. Normally one would need (CB)CT for this but in this chapter a method is presented in which a pseudo-CT image is created based on the planning CT and a deformation field calculated between two US images.

Finally Chapter $\mathbf{7}$ includes a discussion of the presented studies and concludes with a future perspective of US IGRT; the role of US guidance in radiotherapy and the extra capabilities of the US modality which are currently not used in the radiotherapy field.

\section{REFERENCES}

1 Geometric Uncertainties in Radiotherapy: Defining the Planning Target Volume, BIR Working Party. (British Institute of Radiology 2003).

2 J.M. Wilkinson, "Geometric uncertainties in radiotherapy", 2004 Br J Radiol (77:86-87)

3 M. van Herk, P. Remeijer, J.V. Lebesque, "Inclusion of geometric uncertainties in treatment plan evaluation", 2002 Int J Radiat Oncol Biol Phys (52:1407-1422)

4 M. van Herk, "Errors and margins in radiotherapy", 2004 Semin Radiat Oncol (14:52-64)

5 Intensity-Modulated Radiation Therapy - The State of the Art, Edited by Jatinder R. Palta and T. Rockwell Mackie. (Medical Physics Publishing, Madison, WI, 2003).

6 B.G. Fallone, "Intensity-Modulated Radiation Therapy: The State of the Art", 2004 Phys Med Biol (49:357)

7 L.M. Girouard, J. Pouliot, X. Maldague, A. Zaccarin, "Automatic setup deviation measurements with electronic portal images for pelvic fields", 1998 Med Phys (25:1180-1185)

8 A. Bel, P.H. Vos, P.T. Rodrigus, et al., "High-precision prostate cancer irradiation by clinical application of an offline patient setup verification procedure, using portal imaging", 1996 Int J Radiat Oncol Biol Phys (35:321-332)

9 J.E. Schewe, J.M. Balter, K.L. Lam, R.K. ten Haken, "Measurement of patient setup errors using port films and a computer-aided graphical alignment tool", 1996 Med Dosim (21:97-104)

10 D.A. Jaffray, J.H. Siewerdsen, J.W. Wong, A.A. Martinez, "Flat-panel cone-beam computed tomography for image-guided radiation therapy", 2002 Int J Radiat Oncol Biol Phys (53:1337-1349)

11 M. Oldham, D. Letourneau, L. Watt, et al., "Cone-beam-CT guided radiation therapy: A model for on-line application", 2005 Radiother Oncol (75:271-278) 


\section{Chapter 1}

12 U.A. van der Heide, A.N. Kotte, H. Dehnad, et al., "Analysis of fiducial marker-based position verification in the external beam radiotherapy of patients with prostate cancer", 2007 Radiother Oncol (82:38-45)

13 R.D. Foster, T.D. Solberg, H.S. Li, et al., "Comparison of transabdominal ultrasound and electromagnetic transponders for prostate localization", 2010 J Appl Clin Med Phys (11:2924)

14 J.J. Lagendijk, B.W. Raaymakers, A.J. Raaijmakers, et al., "MRI/linac integration", 2008 Radiother Oncol (86:25-29)

15 B.W. Raaymakers, J.J. Lagendijk, J. Overweg, et al., "Integrating a 1.5 T MRI scanner with a 6 MV accelerator: proof of concept", 2009 Phys Med Biol (54:N229-237)

16 A.Y. Fung, K.M. Ayyangar, D. Djajaputra, et al., "Ultrasound-based guidance of intensity-modulated radiation therapy", 2006 Med Dosim (31:20-29)

17 J. Boda-Heggemann, C. Walter, S. Mai, et al., "Frameless stereotactic radiosurgery of a solitary liver metastasis using active breathing control and stereotactic ultrasound", 2006 Strahlenther Onkol (182:216221)

18 J. Boda-Heggemann, F.M. Kohler, B. Kupper, et al., "Accuracy of ultrasound-based (BAT) prostaterepositioning: a three-dimensional on-line fiducial-based assessment with cone-beam computed tomography", 2008 Int J Radiat Oncol Biol Phys (70:1247-1255)

19 J. Boda-Heggemann, P. Mennemeyer, H. Wertz, et al., "Accuracy of ultrasound-based image guidance for daily positioning of the upper abdomen: an online comparison with cone beam CT", 2009 Int J Radiat Oncol Biol Phys (74:892-897)

20 Z. Wei, G. Wan, L. Gardi, et al., "Robot-assisted 3D-TRUS guided prostate brachytherapy: system integration and validation", 2004 Med Phys (31:539-548)

21 J. Stromberg, A. Martinez, J. Gonzalez, et al., "Ultrasound-guided high dose rate conformal brachytherapy boost in prostate cancer: Treatment description and preliminary results of a phase I", 1995 Int J Radiat Oncol Biol Phys (33:161-171)

22 J.H. Youk, E.-K. Kim, M.J. Kim, K.K. Oh, "Sonographically Guided 14-Gauge Core Needle Biopsy of Breast Masses: A Review of 2,420 Cases with Long-Term Follow-Up", 2008 AJR Am J Roentgenol (190:202-207)

23 D. Yan, F. Vicini, J. Wong, A. Martinez, "Adaptive radiation therapy", 1997 Phys Med Biol (42:123-132)

24 Q.J. Wu, T. Li, Q. Wu, F.F. Yin, "Adaptive radiation therapy: technical components and clinical applications", 2011 Cancer journal (17:182-189) 


\section{CHAPTER}

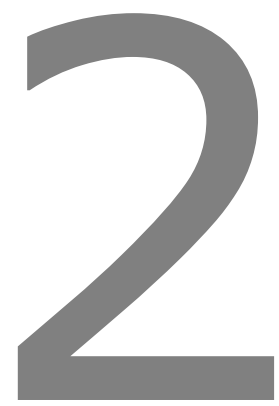

Review of ultrasound image guidance in external beam radiotherapy: I. Treatment planning and inter-fraction motion management

Davide Fontanarosa*, Skadi van der Meer*, Jeffrey Bamber, Emma Harris, Tuathan O'Shea and Frank Verhaegen

*These authors contributed equally to this work.

Published in: Physics in Medicine \& Biology 60: R77-R114, 2015.

DOI: 10.1088/0031-9155/60/3/R77 


\begin{abstract}
In modern radiotherapy, verification of the treatment to ensure the target receives the prescribed dose and normal tissues are optimally spared has become essential. Several forms of image guidance are available for this purpose. The most commonly used forms of image guidance are based on kilovolt or megavolt x-ray imaging. Image guidance can also be performed with non-harmful ultrasound (US) waves. This increasingly used technique has the potential to offer both anatomical and functional information.

This review presents an overview of the historical and current use of two-dimensional and three-dimensional US imaging for treatment verification in radiotherapy. The US technology and the implementation in the radiotherapy workflow are described. The use of US guidance in the treatment planning process is discussed. The role of US technology in inter-fraction motion monitoring and management is explained, and clinical studies of applications in areas such as the pelvis, abdomen and breast are reviewed. A companion review paper $^{1}$ will extensively discuss the use of US imaging for intra-fraction motion quantification and novel applications of US technology to RT.
\end{abstract}




\section{INTRODUCTION}

Radiotherapy (RT) aims to destroy tumor tissue while sparing normal tissue as much as possible. To this end, a therapy plan must be prepared, based on patient anatomy and physical density provided by a simulation (SIM) image. Typically this is a kilovoltage (kV) computed tomography (CT) image, which provides tissue density information for dose calculation $^{2}$. At the planning stage regions of interest (ROI) of the patient are contoured, identifying the target(s) and the organs at risk (OAR). Other imaging modalities, such as positron emission tomography (PET), magnetic resonance imaging (MRI), magnetic resonance spectroscopy (MRS) and ultrasound (US), have been used for planning ${ }^{3-8}$ integrating complementary anatomical, biological and functional information. After identifying target contours they are usually expanded by a margin which accounts for patient-related uncertainties in the RT process 9 , e.g., setup errors, organ motion, and organ volume/shape changes. Margins are selected to minimize the radiation toxicity to the OARs, without introducing a risk of missing the target ${ }^{10}$. The dose distribution is then calculated using a treatment planning system (TPS). The dose is delivered to the patient at the treatment stage (TX) in a certain number of fractions.

At TX, it is paramount to accurately reproduce the position of the patient which existed at SIM, when the plan was prepared. Theoretically, the more accurate the patient positioning system, the more the margins can be reduced, which may lead to less radiation toxicity. By using daily imaging at TX the relatively slow organ movements between daily RT fractions, due to factors such as tumor shrinkage or growth, patient weight loss, and hollow organ filling (referred to as inter-fraction variations), can be corrected.

Different imaging systems have been used to determine the position of the ROI, some can also be used to estimate the volume and shape of the ROI, in an approach referred to as image guided radiation therapy (IGRT). Among these systems, x-ray imaging, currently with electronic portal imaging (EPI) devices and in the past with radiographic film ${ }^{11,12}$, are well established techniques. At TX, bony structures are localized and, if their position is different from their SIM position, a correction is applied to the patient's position. However, soft tissue targets such as the prostate may move with respect to the bones, and standard x-ray imaging may not offer sufficient contrast to image such targets directly. Other techniques have therefore been introduced to deal with this problem, e.g. fiducial markers (FMs) implanted in tissue ${ }^{13}$, cone beam computed tomography (CBCT) $)^{14}$, electromagnetic beacons ${ }^{15}, \mathrm{MRI}^{16}$ and US imaging ${ }^{17}$.

Among the IGRT techniques there is a growing interest in US imaging, which offers rapidly developing two-dimensional (2D), three-dimensional (3D) and four-dimensional (4D) anatomical and functional imaging capabilities for inter-fraction and intra-fraction imaging. Some earlier review papers have dealt with US-based anatomical localization ${ }^{18}$ or 3DUS technology ${ }^{19}$. The current review and its companion review paper ${ }^{1}$ are the first to cover extensively the recent progress and growing applications of modern US imaging in RT. This review will focus on past and new developments of quantitative US imaging at SIM and TX stages, in particular for inter-fraction motion monitoring. After a brief comparison to other IGRT imaging methods, the role of US imaging in the RT workflow will be outlined. This is followed by a brief description of US technology, as employed in RT. The 
role of US imaging in TX planning is described next, followed by a section on its use for monitoring inter-fraction motion. The current applications of US to IGRT for various cancer sites are discussed, and the review concludes with a brief outlook towards the future of US applications in RT. A companion review paper ${ }^{1}$ will focus on the use of US to quantify intra-fraction motion and novel applications of US technology to RT, such as improving target identification with e.g. tissue typing, elastography and contrast imaging, Doppler US imaging, photoacoustography, treatment monitoring, toxicity assessment and dose verification.

\section{BRIEF COMPARISON OF US IMAGING TO OTHER IMAGING MODALITIES}

US imaging is commonly used in radiology mostly as a qualitative diagnostic tool. It has been widely used for cancer diagnosis ${ }^{20}$, it is relatively inexpensive and easy to use, and it can have a diagnostic value comparable to MRI or CT imaging (see e.g. Fuchsjager et al. ${ }^{21}$ ). Table 1 provides a comparison between various imaging systems for IGRT. Since this topic was also extensively covered in a recent review on anatomical imaging methods ${ }^{2}$ we will focus on US-related aspects here.

US imaging allows contouring of soft tissue structures ${ }^{22,23}$ and it can provide complementary information to other imaging systems ${ }^{24}$. US imaging has relatively short acquisition times, comparable to e.g. CT imaging, but it requires currently manual operation of an US transducer in most applications. It is a modality mostly suited for soft tissue imaging; US waves do not propagate easily through air or bones, so applications in lung and brain are uncommon. It is a well-tolerated non-invasive technique, but more invasive applications also exist in RT e.g. in transrectal US imaging for prostate brachytherapy implants ${ }^{25}$. It adds no radiation dose to the patient. It is therefore a good candidate for inter-fraction organ motion monitoring, which is a prerequisite for adaptive applications ${ }^{26,27}$. US imaging suffers to some extent from imaging artefacts. It may provide functional information (e.g. Mace et $a l .{ }^{28}$ ). US is inherently a real-time imaging technology as images are reconstructed and visualized during the scan. Recently, real-time US imaging in 3D became possible ${ }^{29,30}$. With continuous scanning over time, 4D monitoring of tissues during TX is possible ${ }^{31}$. This enables real-time automated structure segmentation and motion compensation during treatment ${ }^{1}$ facilitating adaptive treatment correction. Due to the limited field of view, US images cannot be used directly for treatment planning, but may provide structure contours which may be fused with images from another modality for treatment planning. They lack electron density information, which is required for heterogeneous dose calculation.

US imaging was (and still partially is) considered a user dependent imaging modality with demonstrated inter-user variability ${ }^{32,33}$. The act of US scanning has been reported to cause clinically significant tissue displacement ${ }^{35}$. Moreover, it must be noted that US scanning requires specific skills and training, which are typically not part of the education of the radiation oncology staff. To help resolve these issues, specific scan protocols aimed at minimizing user dependence and excessive transducer pressure on the skin can be 
introduced. To improve quantitative reliability of US systems, advanced transducers, viscous coupling gels, scan directions calculated by computers, speed of sound (SOS) error correction $^{36}$, robotic probe holders, immobile phased arrays, etc. have been introduced. These improvements have made the accuracy and precision of US-based positioning systems more comparable to those of other RT positioning systems.

Using external and intra-cavitary US probes, a wide range of body regions can be imaged: pelvis, abdomen, breast, neck, etc ${ }^{37}$. In clinical practice, only a limited amount of the information provided by US is used, typically the amplitude of the signals and their times of flight (TOF), for brightness and distance calculation. More data could be extracted from e.g. spectral analysis of the US signal ${ }^{38}$. There is therefore much room for future improvements ${ }^{1}$.

\section{US IMAGING IN THE RT WORKFLOW}

From the late 1970s US imaging made a slow entry in the RT environment for planning purposes $^{39}$. About 15 years ago, 2DUS technology was introduced for imaging at the TX stage, primarily with the B-mode acquisition and targeting system (BAT; Nomos, Cranberry Township, PA, USA). At TX, patient setup could be performed based not only on bony structures (as e.g. with megavoltage (MV) CBCT or implanted FM), but on the shape and absolute position in space of the soft tissue targets. This soft tissue based approach had also been reported using kilovoltage CT (KVCT) in the treatment room (CT-on-rail ${ }^{40}$ ). Initially the BAT system was only applied to prostate cancer treatment ${ }^{41}$. Two 2DUS images of the prostate in perpendicular planes were compared to the corresponding SIM CT images. Limitations of this approach have been reported ${ }^{33,41,42}$.

Subsequently, 3DUS systems were introduced allowing acquisition of a 3D volume of the target in absolute coordinates. Other sites were investigated such as breast, liver, neck and cervix/uterus. The current state of the art comprises $4 \mathrm{D}$ real-time tracking of targets. At the time of writing, 4DUS is only available for prostate cancer treatment (Clarity system with Autoscan, Elekta). A mechanically swept transducer continuously scans the target transperineally during irradiation and the position of the prostate is compared to the position of the prostate in the transperineal US image at SIM ${ }^{43-46}$.

In the RT workflow, US imaging in its current implementations is used at two stages. A summary of the steps in the RT workflow, which can benefit from US imaging is displayed in figure 1. In the initial steps, US can help with contouring targets and designing treatment plans, especially if functional information is available ${ }^{1}$. At TX, US can provide information about target positions before treatment or during treatment delivery ${ }^{1}$. This information can be used for setup corrections in IGRT or to update the dose distribution and, based on the comparison between the present and the expected dose distributions, dose guided radiotherapy (DGRT) can be performed ${ }^{47}$. Finally, functional information can be used for treatment response assessment and follow-up ${ }^{1}$. 
Table 1: Comparison between US and the most frequently used IGRT techniques in RT. CT refers to devices specific for RT (such as CBCT or CT on rails). Two cases are distinguished: when the beam is in the $k V$ range (standard $C T$ and $k V$ cone beam $C T$ ( $k V-C B C T)$ ) and when it is in the MV range (MVCBCT and Tomotherapy). ART refers to adaptive RT ${ }^{34}$. MRI refers to the MR-LINAC combination, which was not available for clinical use at the time this review was written.

\begin{tabular}{|c|c|c|c|c|}
\hline & \multicolumn{4}{|c|}{ Imaging modality } \\
\hline & CT & US & MRI & Portal Imagers \\
\hline $\begin{array}{l}\text { Acquisition time } \\
\text { (including setup/ } \\
\text { preparation) }\end{array}$ & $\sim 2 \min$ & $\sim 2 \min$ & $5 \mathrm{~min}$ & $<1 \min$ \\
\hline Spatial resolution & $1-3 \mathrm{~mm}$ & Sub-mm & $0.5-5 \mathrm{~mm}$ & $\sim 1 \mathrm{~mm}$ \\
\hline $\begin{array}{l}\text { Visualization } \\
\text { capabilities }\end{array}$ & $\begin{array}{l}\text { KVCT: soft tissue } \\
\text { in the whole body. } \\
\text { Whole body imaging } \\
\text { MVCT: bone } \\
\text { contrast only }\end{array}$ & $\begin{array}{l}\text { Soft tissues: high } \\
\text { contrast } \\
\text { Difficult to image } \\
\text { through air or bones } \\
\text { (no lungs/brain) }\end{array}$ & $\begin{array}{l}\text { Soft tissue: high } \\
\text { contrast }\end{array}$ & $\begin{array}{l}\text { 2D, Poor } \\
\text { contrast (only } \\
\text { Bones and FM) }\end{array}$ \\
\hline Invasiveness & $\begin{array}{l}\text { YES with FM or } \\
\text { contrast }\end{array}$ & $\begin{array}{l}\text { YES for some } \\
\text { applications (in- } \\
\text { tracavitary) or using } \\
\text { contrast }\end{array}$ & $\begin{array}{l}\text { YES for special } \\
\text { application with } \\
\text { contrast }\end{array}$ & $\begin{array}{l}\text { YES with FM or } \\
\text { contrast }\end{array}$ \\
\hline Dose delivered & $\begin{array}{l}1-3 \text { cGy (kV); } \\
1-15 \text { cGy (MV) }\end{array}$ & None & None & $\begin{array}{l}<1 \text { cGy (kV); } \\
6 c G y(M V)\end{array}$ \\
\hline $\begin{array}{l}\text { Operator de- } \\
\text { pendence }\end{array}$ & NO & YES & NO & NO \\
\hline $\begin{array}{l}\text { Intra-fraction } \\
\text { monitoring }\end{array}$ & NO & YES & $\begin{array}{l}\text { YES } \\
\text { (Semi real-time) }\end{array}$ & $\begin{array}{l}\text { YES } \\
\text { (fluoroscopy) }\end{array}$ \\
\hline Image distortion & $\begin{array}{l}\mathrm{CT}: \text { No; } \mathrm{CBCT} \text { : } \\
\text { scatter distortion }\end{array}$ & $\begin{array}{l}\text { YES } \\
\text { (probe pressure) }\end{array}$ & $\begin{array}{l}\text { YES for metal } \\
\text { parts }\end{array}$ & NO \\
\hline Imaging artefacts & $\begin{array}{l}\text { Streaking, beam } \\
\text { hardening }\end{array}$ & Aberrations & $\begin{array}{l}\text { Geometrical } \\
\text { uncertainties } \\
\text { depending on } \\
\text { acquisition type }\end{array}$ & $\begin{array}{l}\text { Streaking } \\
\text { (metal); } \\
\text { low SNR (MV) }\end{array}$ \\
\hline $\begin{array}{l}\text { Functional/ } \\
\text { biological infor- } \\
\text { mation }\end{array}$ & YES & YES & YES & NO \\
\hline Motion tracking & $\begin{array}{l}\text { YES (4D-(CB)CT); } \\
\text { with poor soft tissue } \\
\text { contrast; needs FM }\end{array}$ & YES & YES (2D) & $\begin{array}{l}\text { YES } \\
\text { (fluoroscopy) }\end{array}$ \\
\hline $\begin{array}{l}\text { Scan setup/ } \\
\text { preparation }\end{array}$ & NO, unless contrast $\mathrm{CT}$ & YES & $\begin{array}{l}\text { NO, unless } \\
\text { Contrast MRI }\end{array}$ & NO \\
\hline ART & YES & YES & YES & YES \\
\hline Dose calculation & YES & NO & NO & YES \\
\hline $\begin{array}{l}\text { Attenuation } \\
\text { information for } \\
\text { dose calculation }\end{array}$ & YES & NO & NO & YES \\
\hline
\end{tabular}




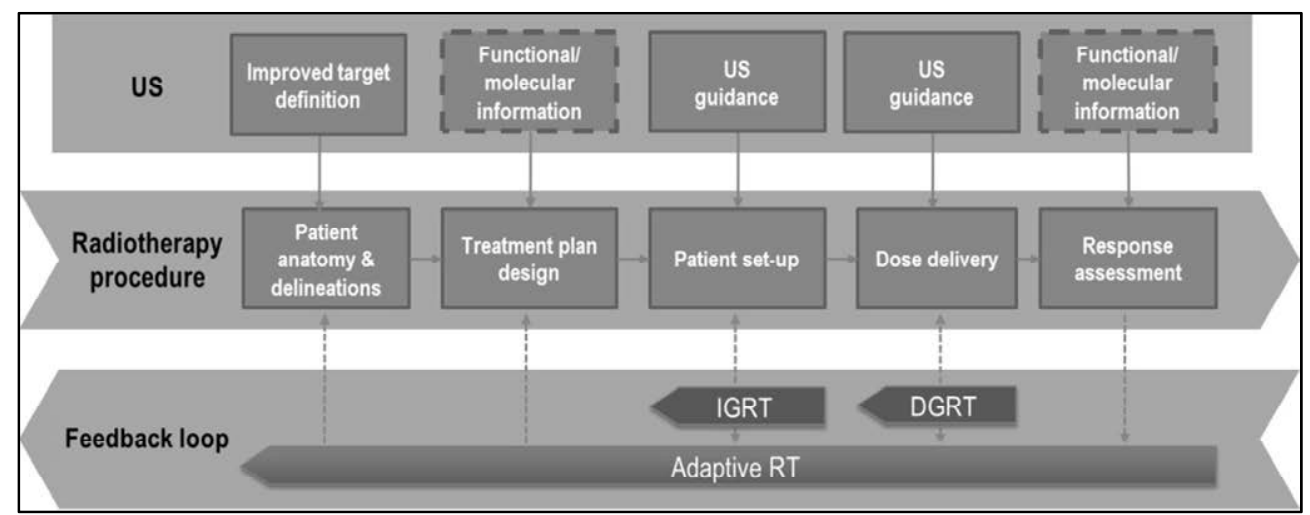

Figure 1: US imaging in the RT workflow. The middle row shows the steps involved in RT. The large arrow indicates the temporal direction. The top row identifies the type of input which can be provided by US imaging. Boxes with dashed outlines are the applications not yet clinically commonly available. The bottom row shows the feedback loops which can be assisted by US: patient positioning or treatment plans can be adapted based on the new position of targets provided by US (IGRT) or based on the comparison between present recalculated dose distribution and the expected dose distribution, calculated at SIM (DGRT).

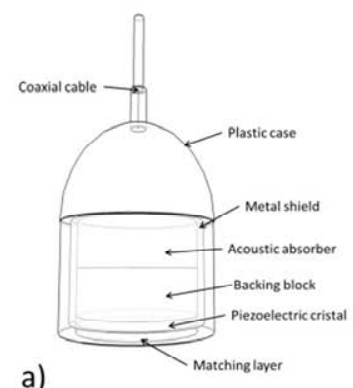

a)

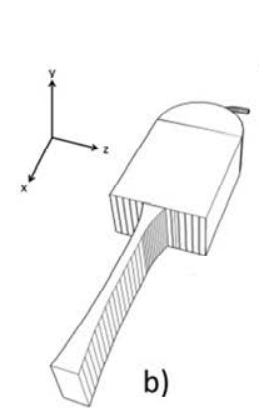

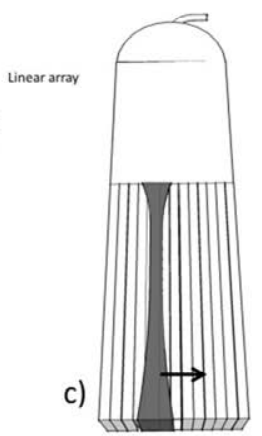

Figure 2: (a) A single element US probe with its components. (b) A linear array transducer with indication of a line of view and the three directions corresponding to axial $(X)$, elevational $(Y)$ and lateral (Z) US beam dimensions from a single acoustic aperture. (c) The field of view of a linear probe; a single line of view (A-mode) is highlighted and the scanning direction is indicated by an arrow. (d) A curvilinear probe with the field of view divided in its lines of view. (Images adapted with permission from figures 16.8 and 16.35 in Bushberg ${ }^{48}$.)

\section{TECHNOLOGIES}

Many references are available in the literature about US physics theory and technology. Among these, Carson ${ }^{49}$, Wild ${ }^{50}$, Beranek and America ${ }^{51}$, Bushberg ${ }^{48}$, Duck et al. ${ }^{52}$, Webb $^{53}$, 
Bamber et al. ${ }^{54}$, Hill and Dunn ${ }^{55}$ and Hill et al. ${ }^{56}$ offer an overview at different levels of complexity.

\section{Transducers}

An US transducer converts electrical signals into acoustic waves and vice versa. Figure 2(a) shows some commonly employed types in RT. The piezoelectric elements of the transducer can be activated in different ways. Subsets of the array can be fired simultaneously to create an acoustic aperture (line of view); then multiple sequential apertures create the field of view. Alternatively, the elements may be activated at different times to steer the beam (phased mode). We refer to the aforementioned literature for more details.

\section{Image formation}

In US imaging for RT the frequencies used are typically in the 2-8 $\mathrm{MHz}$ range. Modern US transducers for medical applications work in pulse-echo mode: the transducer both emits and receives the US waves. If in the scanned volume there is a variation in the physical properties of the tissues (for example density or elasticity), then the acoustic impedance changes. Acoustic impedance is defined as:

$$
Z=\rho c
$$

where $\rho$ is the physical density and $c$ is the SOS. Whenever a pulse of US waves encounters an interface between materials or tissues with different $Z$, a proportion of the energy of the pulse is reflected. The reflections are recorded as echoes and used to create the US image. Echo times (or TOF) and amplitudes are recorded. Then the TOFs are converted to depths assuming a fixed value for the SOS (typically $1540 \mathrm{~m} \mathrm{~s}^{-157}$ ). Finally the echo amplitudes are plotted on a screen as function of depth. Brightness mode (B-mode) scanning is the most commonly used in RT. The reader is again referred to the literature for more details.

\section{DUS imaging}

Conventional 2DUS images are scanned at arbitrary angles through the body. Multiple images are then combined by the operator to form a mental image of the 3D anatomy. This process depends heavily on the operator's skills and is inherently qualitative. For quantitative applications, such as measurement of organ shapes or volumes, this is not optimal $^{19,58}$. For these applications the limitations of 2DUS imaging can be overcome with 3DUS imaging ${ }^{9,59}$. From the first attempts in diagnostic imaging ${ }^{60-62}$ technological advances led to the development of the modern commercial 3DUS systems. Currently, three major approaches exist: mechanical scanning, free-hand scanning with position sensing, and electronic scanning which requires $2 \mathrm{D}$ matrix arrays.

In the first category are those devices where a motorized mechanism inside the probe case translates, tilts or rotates a conventional 2D imaging transducer, while an acquisition system records the images. Then these images are used to reconstruct 3D volumes using the information on their relative positions provided by a mechanical encoder. The sampling angle can usually be adjusted to balance image quality with temporal resolution ${ }^{63}$. In 
the second approach, tracking sensors are mounted on conventional 2DUS scanners which allow the measurement of the transducer's position and orientation while it is scanning the volume. This information is then coupled with the images to reconstruct a 3D volume $^{64}$. The most commonly used tracking systems are robotic arms ${ }^{65}$, acoustic sensors $^{66}$, optical sensors ${ }^{67}$, magnetic sensors and speckle decorrelation ${ }^{68}$. In the third approach a static 2D matrix of transducer elements is used ${ }^{69}$ to perform direct volume scanning ${ }^{70}$, which is a 3D scan of a volume of interest in its totality, within a time in which movement is negligible and with sufficient spatial resolution. 2D matrix arrays are not yet in common use in RT.

Visualizing the acquired volumes is a key point in 3DUS. The most frequently used visualization techniques are multi-planar reformatting (MPR) and surface rendering. In MPR, it is possible to browse through the volumes selecting e.g. a couple of intersecting cross planes $^{71}$ (figure $3(a)$ ). Surface rendering is based on ray-casting techniques. It is used when a high contrast is present between different tissues, allowing surface reconstruction of e.g. fetal images ${ }^{72}$ (figure $3(b)$ ) or cardiac images ${ }^{73}$. Also volumetric rendering (VR) was introduced using techniques such as maximum intensity projection ${ }^{74}$.

In RT, typically a 3DUS scan is performed before the treatment and compared to either the SIM CT scan or a SIM 3DUS scan. This allows a direct comparison of the anatomy, as described in the introduction.

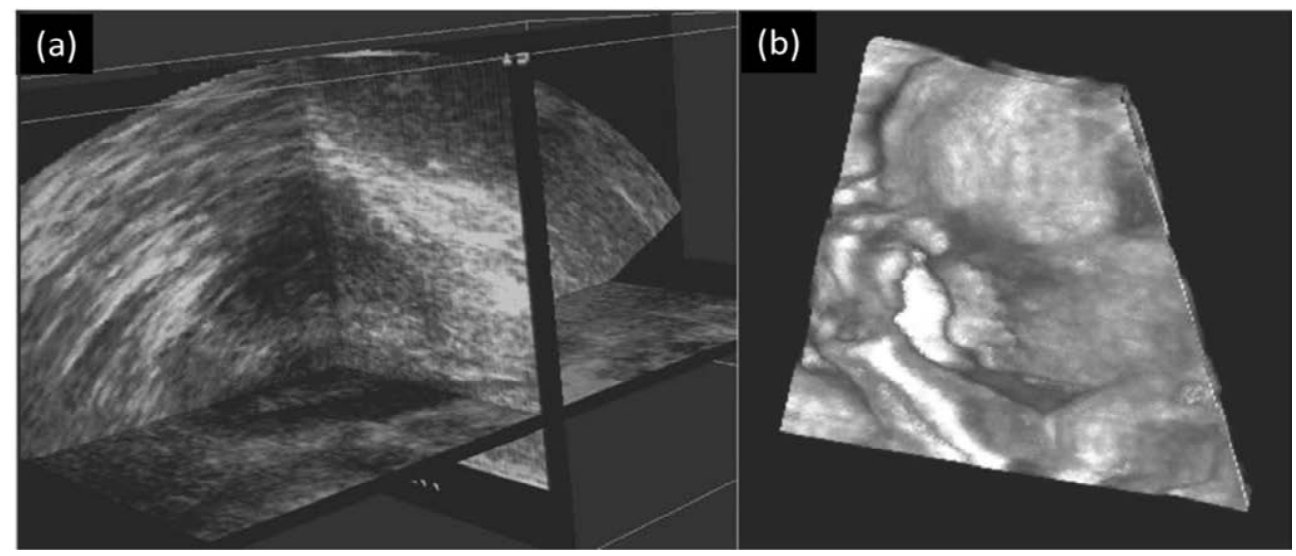

Figure 3: (a) Example of MPR view of a prostate, showing intersecting extracted planes. (b) Example of 3DUS surface render of the face of a fetus. (Images reproduced with kind permission of Fenster et $\left.a l .{ }^{75}\right)$.

\section{Quantitative measurements in RT using US: errors due to image aberrations}

In RT we require quantitative measurements of position, volume and shape of anatomical structures. Minimizing or eliminating image aberrations is therefore of importance. Different causes can generate aberrations. As detailed in the following paragraphs, they can be due to the US device used, the operator scan technique or to US physics. Frequently aberrations appear as obvious distortions or unexpected periodic symmetries. In these cases, correction strategies can be applied. But sometimes aberrations, such as global or 
local image scale error, can be very difficult to detect, because the effect produced has no obvious characteristics.

Distortion of the propagation direction due to acoustic refraction can cause image aberrations. Multiple reflections can cause reverberation. Mirror images may be induced by strong reflectors in the beam path. Objects with very high or very low echogenicity can cause enhancement or shadowing behind the structure. If excessive pressure is applied by the probe, this can cause displacement and deformation of the scanned structures. US wave propagation along directions other than the assumed beam axis (axial) is caused by side lobes or grating lobes. Finally, an aberration which is particularly difficult to detect is due to the assignment of incorrect SOS values, either globally or locally. Most US devices assume that the SOS value is constant in human soft tissues at a value of $1540 \mathrm{~m} \mathrm{~s}^{-1}$. This value ranges from $1450 \mathrm{~m} \mathrm{~s}^{-1}$ (fat) to $1700 \mathrm{~m} \mathrm{~s}^{-1}$ (tendons) ${ }^{76,77}$. Since the positions of structures are calculated using SOS, the discrepancy between the assumed and the real local SOS values can produce errors in localization of structures. These distance conversion errors accumulate with depth away from the skin.

\section{Resolution and contrast}

Linear scans produce rectangular images where the lines of view (a-lines) have good correspondence with the vertical columns of pixels in each 2D image. For sector scans produced with curvilinear probes or phased arrays the image has a fan shape. Typically the image resolution improves with depth up to the depth of the focal zone. The spatial resolution can be further divided into three types:

The axial (or longitudinal) resolution is the ability to distinguish objects spatially close along the direction of propagation of the beam ( $X$ direction in figure $2(b)$ ). Axial resolution depends on the spatial pulse length (SPL). If the separation in the longitudinal direction between two reflectors is smaller than $1 / 2$ SPL then the reflected pulses overlap and the reflectors are unresolved. For example, at $5 \mathrm{MHz}$ frequency a typical pulse composed of three cycles has a SPL of $0.93 \mathrm{~mm}$. This means that the best resolution achievable is $1 / 2(0.93 \mathrm{~mm})=0.47 \mathrm{~mm}$.

The lateral resolution is in the direction perpendicular to the axial direction but within the scan plane ( $Z$ direction in figure $2(b))$. The lateral resolution depends on the beam diameter; it varies with depth, potentially resulting in a depth-dependent image quality. It is the best in the focal spot, where the beam reaches maximum convergence. The typical lateral resolution for an unfocused transducer is approximately 2 to $5 \mathrm{~mm}$.

The elevational resolution is in the third orthogonal direction ( $Y$ direction in figure $2(b)$ ). Elevational resolution is dependent on the transducer element height in much the same way that the lateral resolution is dependent on the transducer element width.

The side/grating lobes are also different for different transducer types and must be carefully investigated before making quantitative evaluations.

\section{Spatial calibration}

For RT applications it is necessary that the relationship between the US image coordinates and the treatment room coordinates is known. This requires accurate knowledge of the US 
probe position and orientation. To this end, clinical US devices have used mechanical arms or optical tracking. For the former, before each use the gantry is put at a known position and the transducer is placed in a holder attached to the gantry. The mechanical arm tracks the movement of the US transducer and software is used to determine its position (e.g. BAT system). For optical tracking, infrared (IR) light emitting diodes (LEDs) or reflectors are attached to the probe to localize the probe position via room-mounted optical cameras and to retrieve the corresponding room position of the US images (e.g. BAT and Clarity systems). Both systems require spatial calibration to relate the probe position to treatment room coordinates. Bouchet et al. ${ }^{78}$ developed a method of spatial calibration for both $2 \mathrm{D}$ and $3 \mathrm{D}$ systems. A specially designed phantom containing nylon wires used to generate references for spatial localization was used to calibrate a 3DUS system with a reported mean accuracy of $0.2,0.9$ and $0.6 \mathrm{~mm} \mathrm{AP}$, lateral and axial. Poon and Rohling ${ }^{79}$ compared three calibration methods for 3DUS transducers. A phantom, with nylon wire shaped as the three letters 'IXI', was found to produce the best results with accuracy of $2.15 \mathrm{~mm}$ and reproducibility of $1.50 \mathrm{~mm}$.

Schlosser et al. $^{80}$ have developed a fully automated calibration method that does not rely on customized calibration phantoms. It can be used to spatially localize (inter-fraction) and track (intra-fraction) the probe during RT treatments with a reported accuracy of 1.5 $\mathrm{mm}$ (RMSE).

\section{TREATMENT PLANNING}

\section{Target volume delineation}

Presently, US imaging is used for target delineation in RT mainly for prostate gynecological and breast cancers, although other sites are sometimes imaged such as liver, kidney, pancreas $^{81}$ and lymph nodes in head and neck patients ${ }^{82}$. Prostate delineation can benefit particularly from US imaging because the organ is located beneath the bladder which, when full, creates an easily recognizable bladder-prostate interface due its low echogenicity. Also, seminal vesicles can be properly identified, especially when transrectal (TRUS) or transperineal US systems are used. Extensive literature is available about (semi-) automated segmentation methodologies for prostate (see Ghose et al. ${ }^{83}$ for a review). These techniques can be classified into different categories: contour- and shape-based methods; region-based methods; supervised and unsupervised classification methods; and hybrid methods. Even if 3D volumes are available, most of the algorithms are based on 2D projections $^{84-86}$, possibly using information from nearby slices ${ }^{87}$. But since the introduction of 3D systems, more sophisticated algorithms are being developed. Also US-based functional imaging is gaining popularity as a source of information for target identification. For example, contrast enhanced US (CEUS) can be used to detect pathological angiogenesis inside the prostate ${ }^{88}$. Also, other biomarkers of cancer can be imaged with US techniques ${ }^{89}$. The information can then be used to create a biological target volume (BTV).

For gynecological applications (endometrial or cervical cancer) target definition is generally complex due to the presence of multiple structures, which move and deform 
significantly during treatment. US images typically cannot show the entire target but, if the bladder is full, uterus, cervix and vagina can be correctly visualized ${ }^{90}$.

\section{Approaches to multi-modality image registration}

Registration of US images to complementary images from other modalities can be of use for treatment plan contouring. The methods available for image registration in the literature can be classified into two categories: rigid and non-rigid. Until now, prostate cancer has been the most important application of US guidance in RT and image registration techniques were mainly focused on this organ. Some work was also published on the liver $^{91}$, where a rigid registration between US and MR liver images was based on major vessels as template matching points. Deformable registration of the liver using a model of respiratory motion has been reported ${ }^{92}$. In this study, US was fused to both MR or CT scans.

Under some circumstances non-rigid registration methods need to be applied to compensate for prostate deformation ${ }^{93,94}$. This can occur because of multiple factors: e.g. the displacement of tissue by the endorectal coil during MR scanning, and rectum or bowel filling. Typically, before a deformable registration is applied, an initial manual or automatic affine alignment is required. The most used transformations are spline-based ${ }^{94}$, in particular octree splines ${ }^{95}$ or B-splines. These techniques are not yet reliably established in RT, so most of the deformable registration examples are found outside of the external beam RT field $^{96-98}$. The transformation can also be based on deformable models, such as the adaptive focus deformable model ${ }^{99}$ and elastic warping ${ }^{100}$, which were used for localization of prostate biopsy targets ${ }^{101}$. A registration based on biomechanical finite element modelling was proposed in Hu et al. ${ }^{102,103}$. In Ding et $a .^{87}$, magnetic resonance spectroscopic imaging (MRS) image volumes were registered to US/CT images with the assumption that any position inside the prostate did not change with respect to the prostate contour and the center of mass. In general, one of the major issues connected with deformable registrations based on US is the limited FOV which does not allow a complete match with the other modalities (such as CT or MRI), and this results in the need for assumptions on how the deformation propagates outside the US volume.

\section{Assessment of organ motion}

At this moment, $4 \mathrm{DCT}^{104}$ is commonly used to determine the internal target volume, accounting for e.g. the breathing motion in the PTV. US could also be a useful image modality to quantify this motion. US inter-fraction motion monitoring will be discussed in a later section. Monitoring and tracking of the intra-fraction motion ${ }^{105}$ will be discussed in the second part of this review (O'Shea et al. $\left.{ }^{1}\right)$. 


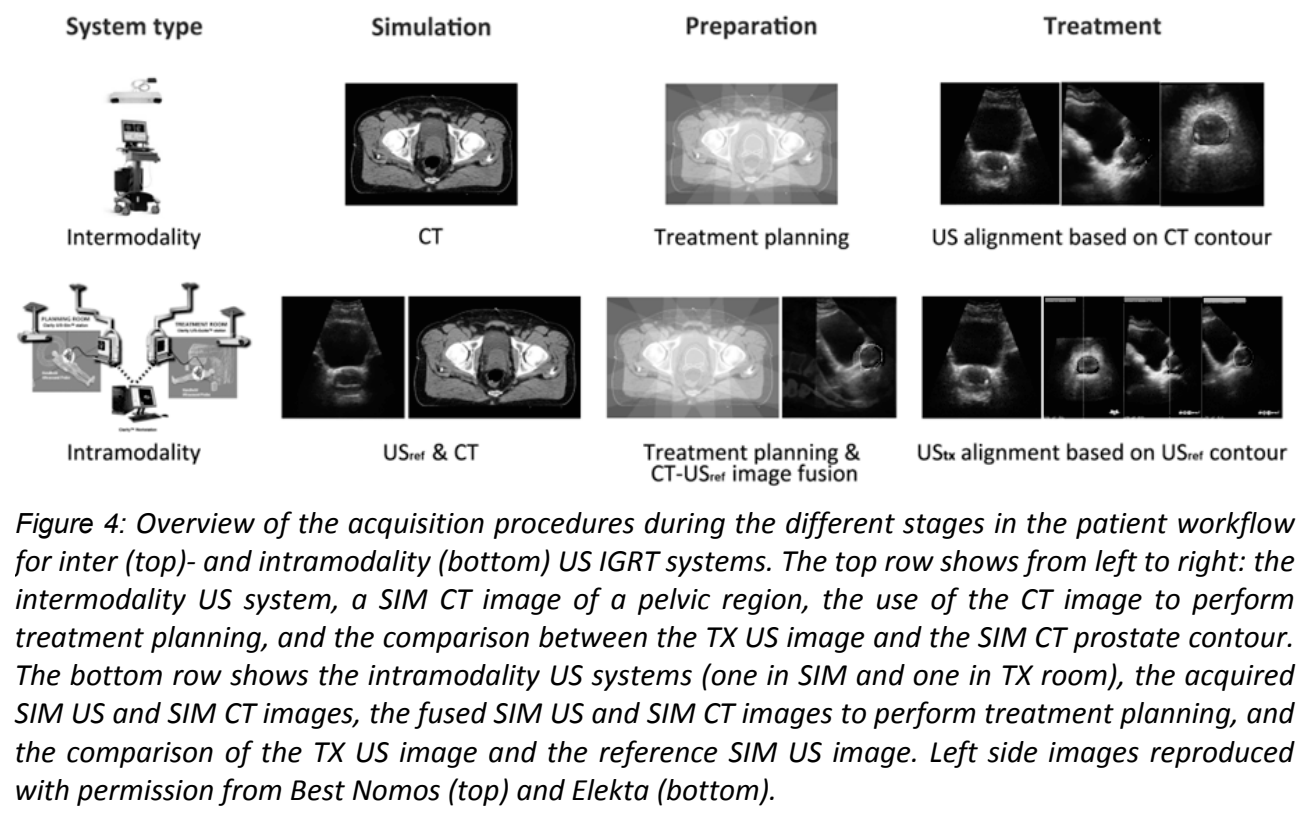

\section{INTER-FRACTION MOTION MONITORING}

\section{Intermodality systems and intramodality systems}

US IGRT systems can be divided in two categories: intermodality and intramodality systems (figure 4). Intermodality systems compare the contours of the reference CT image, acquired during SIM stage, to US images acquired at the TX. The intramodality approach compares like with like, which means it compares the treatment US image contour with an US contour derived from a reference US image acquired at the time of CT SIM.

The first generation US IGRT systems were 2D intermodality systems. An example is the B-mode Acquisition and Targeting (BAT) system (Best Nomos, Pittsburgh, PA, USA) of the late 90s. Later 3DUS imaging systems, like the SonArray system (Varian Medical Systems, Palo Alto, CA) from the early 2000s frequently used the intermodality approach. The Clarity system (Elekta, Stockholm, Sweden), which is a quantitative intramodality 3DUS IGRT system, appeared around 2004. It is interesting to note the concurrent research outside of the IGRT field on intermodality 3DUS image registration, and commercial development of systems for image guided surgery and biopsy, where similar technologies to those described above are used to monitor the position and orientation of the US probe $^{106-109}$.

Intermodality systems are implemented at the TX stage of the clinical workflow. The US images are acquired prior to each treatment fraction, and at the matching stage their contours need to be aligned with the delineated target contours of the planning CT. Because the US image guidance workflow is restricted to the TX stage, only one US system has to be calibrated, and any uncertainties arising from image fusion are limited to one 
stage. Tissue boundaries may be displayed differently in CT and US, resulting in different CT and US contours ${ }^{18,22,110,111}$. Therefore, even with proper training, aligning US images to CT contours can be challenging and error prone. SOS image aberrations due to use of incorrect $\mathrm{SOS}^{36,112-115}$ can lead to geometrical differences between the CT and US. To reduce the inter-imaging modality discrepancies Task Group 154 of the American Association of Physics in Medicine ${ }^{116}$ recommends integration of US reference images during SIM, i.e. they advocate the use of an intramodality system.

With an intramodality system the US image guidance workflow begins at the SIM stage. Either immediately before or after the CT acquisition a reference US is acquired. During subsequent TX fractions, at the matching stage the contours from this reference US are aligned with the daily US images. Although the TX step of the intramodality system is less sensitive to alignment errors, the complete workflow contains extra registration procedures, which may increase the susceptibility of the patient positioning process to error. Proper training, accurate calibrations, strict quality control, and good understanding of the complete process can contribute to a decrease of this error susceptibility.

In addition to the commercially available systems, some researchers are developing their own solutions ${ }^{80,117-122}$. Table 2 gives an overview of the capabilities of these systems (intra-fraction motion tracking is discussed in part II of this review ${ }^{1}$.

Table 2. Commercial (first 4 rows) and non-commercial (last row) US systems for inter-fraction motion management in RT.

\begin{tabular}{|c|c|c|c|}
\hline System or authors name & Image type & Modality type & Remarks \\
\hline $\begin{array}{l}\text { SonArray system } \\
\text { (Varian Medical Systems, } \\
\text { Palo Alto, CA, USA) }\end{array}$ & $3 \mathrm{D}$ & $\begin{array}{l}\text { Intermodality, } \\
\text { inter-fraction } \\
\text { motion }\end{array}$ & $\begin{array}{l}\text { Tome et al. }{ }^{123} \\
\text { No longer available }\end{array}$ \\
\hline $\begin{array}{l}\text { BAT/BATCAM system } \\
\text { (Best Nomos, Pittsburgh, } \\
\text { PA, USA) }\end{array}$ & $2 \mathrm{D} / 3 \mathrm{D}$ & $\begin{array}{l}\text { Intermodality, } \\
\text { inter-fraction } \\
\text { motion }\end{array}$ & www.nomos.com/products_Bat.html \\
\hline $\begin{array}{l}\text { Clarity system } \\
\text { (Elekta, Stockholm, Sweden) }\end{array}$ & $3 D$ & $\begin{array}{l}\text { Intramodality, } \\
\text { inter-fraction } \\
\text { motion }\end{array}$ & www.elekta.com/clarity \\
\hline $\begin{array}{l}\text { Clarity Autoscan with } \\
\text { Monitoring (Elekta, Stock- } \\
\text { holm, Sweden) }\end{array}$ & $4 \mathrm{D}$ & $\begin{array}{l}\text { Intramodality, } \\
\text { inter-fraction } \\
\text { motion, trans- } \\
\text { perineal }\end{array}$ & $\begin{array}{l}\text { Lachaine and Falco }{ }^{31}, \\
\text { www.elekta.com/clarity }\end{array}$ \\
\hline Kaar et al. ${ }^{122}$ & $3 D$ & $\begin{array}{l}\text { Intramodality, } \\
\text { inter-fraction } \\
\text { motion }\end{array}$ & $\begin{array}{l}\text { Gantry head mounted clamp for the } \\
\text { US-probe, automatic registration }\end{array}$ \\
\hline
\end{tabular}

\section{Practical implementation}

To implement US IGRT, different aspects have to be investigated. Primarily, the US system itself has to be accurate ${ }^{22,118,123-125}$. One of the major concerns for US IGRT is the user variability. Not only is the acquisition of the image for most US systems still performed manually, the images may also be more difficult to interpret than e.g. a CT or MR image. 
The complete body contour is not visible on an US image, therefore structures may be more difficult to identify on an US image. User experience and training have been shown to improve the consistency and reproducibility of US image interpretation among users ${ }^{126,127}$.

In most US systems there are several time points at which the user variability can have an influence; during image acquisition, structure contouring and at both the inter- and intramodality matching stages. For prostate ${ }^{126,128-131}$, breast ${ }^{132}$ and liver ${ }^{133}$ some of the variabilities have been investigated (table 3 ) and can therefore be taken into account in the uncertainty of the whole procedure.

One source of large user variability during image acquisition may be probe pressure. Several studies have shown a potential influence of probe pressure on the prostate position. In two studies ${ }^{134,135}$ radiographs of brachytherapy seed implants were used to measure the influence of probe pressure. Radiographs were acquired before/after and during US image acquisition/SIM. Another study ${ }^{128}$ used sequential CT scans and a dummy US probe. There were also studies which only used the US modality to measure the prostate displacement $^{35,131}$. The maximum reported prostate displacements due to probe pressure ranged from no displacement to $5 \mathrm{~mm}$ under normal conditions and up to $10 \mathrm{~mm}$ if (inappropriately) high pressure was applied. The average prostate displacement for the clinically applied pressure is around $3 \mathrm{~mm}^{35,128,131}$. For intramodality systems, in theory the probe pressure distortion is minimized when similar scanning procedures are used to acquire the images during SIM and TX stages; the corrective shifts are derived as differences between two images, both affected by (ideally) the same probe pressure. One group $^{136}$ reported that it might be difficult to reproduce the applied pressure from one session to another due to the manual sweeping acquisition technique. In general, scanning with similar probe placement, minimal pressure and high viscosity coupling gel may help to minimize the problem. In 2002 a solution was suggested to correct the images mathematically for the probe pressure ${ }^{119,137,138}$. Probe pressure can be avoided completely by scanning the prostate transperineally, e.g. by the Clarity Autoscan system ${ }^{31}$. Another

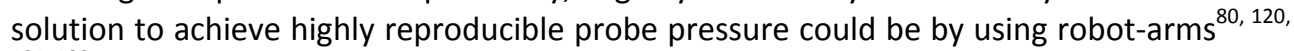
121,139 .

In addition, the position measured by the US system has to be compared to the 'true' position of the target. This was investigated in many studies (table 6). Unfortunately, the 'true' position of an organ is difficult to determine, and none of the available localization techniques are true gold standards. Most studies compare the localization from US to another IGRT technique, where usually the reference technique is already clinically implemented. Some studies reported clinically significant differences. This should not immediately lead to the conclusion that the US IGRT system is incorrect. Nonetheless, with clinically significant differences between systems, changing from one system to another can result in a better or worse patient treatment.

For breast and prostate treatments, the effects of acquiring US images on the daily treatment workflow are minimal. All US images can be acquired by the technologists and no physician involvement is necessary. Extra time for US scans may be offset by time saved by omitting the FM implantation. The time necessary to accomplish the complete US workflow in the treatment room (acquire the TX US, matching to SIM US or CT and shifting the patient) can, after proper training, be less than $5 \min ^{140-142}$. 


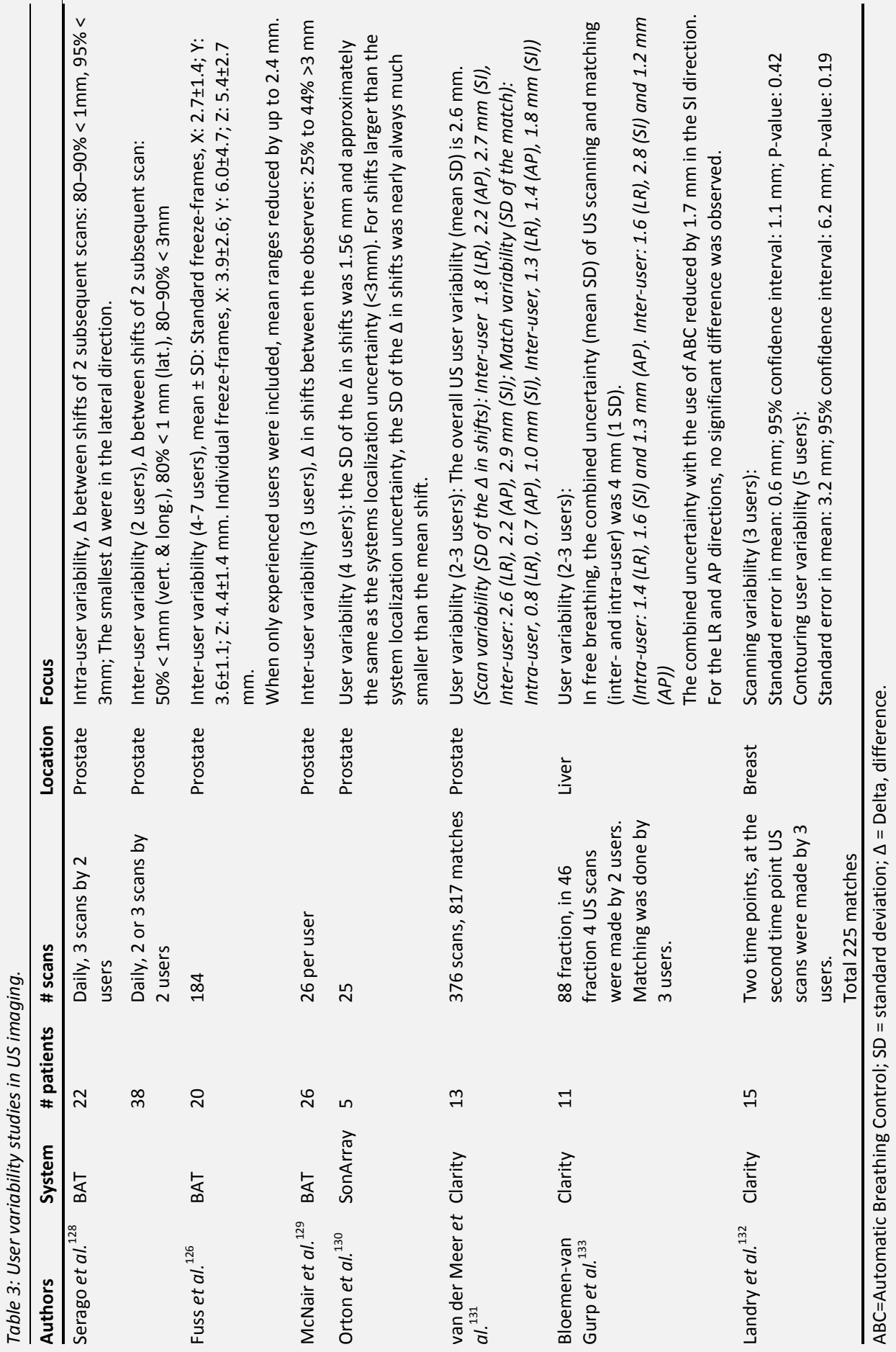




\section{Comparisons with other IGRT modalities}

Various other imaging methods have been investigated to localize the target during RT treatment, as discussed in a previous section. In general, one could differentiate systems by their use of ionizing radiation or not, or by their soft-tissue contrast. The non-ionizing radiation techniques, US and MRI, have in most cases high soft tissue contrast. In CT and CBCT the soft tissue contrast is moderate, and in portal imaging only objects with high densities such as bones are easily visible. To compensate for lack of contrast, implanted FMs with a high density can be used as a surrogate to localize the position of the soft tissue organs.

From a practical point of view, $\mathrm{CBCT}$ and portal imagers have the benefit of being fully integrated in most linacs. The integration of MRI and a linac is under development ${ }^{143}$, but in-room solutions for both $\mathrm{MRI}^{144,145}$ and $\mathrm{CT}^{146}$ are already clinically available. This, however, requires large treatment rooms. US imaging systems are less space consuming and less costly, but require patient contact and a user or robot-arm in the treatment room. In the following sections the requirements for imaging of several target sites will be discussed in more detail.

For prostate, the one-to-one comparison of US with other IGRT techniques are shown in table 6.

\section{CLINICAL STUDIES}

\section{Prostate}

Current prostate RT uses fractionated, dose escalating, Intensity-Modulated RT techniques (IMRT), i.e. steep dose fall-offs and tight target margins. The prostate, however, is known to be a structure which can move from day to day by up to several $\mathrm{mm}$ or even $\mathrm{cm}$ in an unpredictable way, mainly due to variations in rectal and bladder filling ${ }^{147,148}$. This necessitates the accurate determination on a daily basis of the position of the prostate and the OARs that caused it to move prior to RT treatment. An US study of the inter-fraction prostate displacement from the planning position found an average 3D displacement of about $7.8 \mathrm{~mm}^{17,}{ }^{149}$. When analyzed in each dimension, 31-38\% of displacements were greater than $5 \mathrm{~mm}^{150}$. In another study ${ }^{151}$, systematic uncertainties showed that nonuniform PTV margins of $2.7 \mathrm{~mm}$ anterior, $14.9 \mathrm{~mm}$ posterior, $7.7 \mathrm{~mm}$ right, $6.7 \mathrm{~mm}$ left, 11 $\mathrm{mm}$ superior, and $7 \mathrm{~mm}$ inferior are necessary to encompass the prostate for $95 \%$ (2 SD) of all displacements if the US localization system were not used. Fung et al. ${ }^{17,149}$ observed a slight trend toward superior-posterior directions in the scatter plots of the shifts. In the absence of systematic uncertainties, a uniform PTV margin of $9 \mathrm{~mm}$ would suffice ${ }^{151}$. According to another US study ${ }^{15}$, a 4 mm PTV margin would be sufficient.

\section{Prostate imaging modalities in RT}

For prostate RT, a commonly used IGRT method is MV EPI in combination with implanted FMs as a surrogate for the prostate location ${ }^{13}$. FM implantation is not always feasible or 
without risks; infections and bleeding ${ }^{152,153}$ are well known complications in this procedure ${ }^{154,155}$. Superior to imaging surrogate FMs are techniques in which the prostate itself is visualized. Both $\mathrm{CT}$ and $\mathrm{CBCT}$ have moderate soft tissue contrast, reducing the capability to distinguish the prostate from surrounding normal tissue. MRI and US have a higher soft tissue contrast, facilitating prostate recognition. Table 4 compares different IGRT imaging modalities.

Table 4: Comparison of prostate imaging modalities.

\begin{tabular}{|c|c|c|}
\hline System & Advantages & Disadvantages \\
\hline $\begin{array}{l}\text { Implanted electro- } \\
\text { magnetic tran- } \\
\text { sponders }\end{array}$ & Real-time tracking & $\begin{array}{l}\text { Invasive, risk of infection/bleeding, transponder } \\
\text { migration. Causes artefacts on post-treatment MRI. }\end{array}$ \\
\hline kV or MV EPI $\pm F M$ & $\begin{array}{l}\text { Quick and easy to } \\
\text { acquire. Bones and } \\
\text { FM easily visualized. }\end{array}$ & $\begin{array}{l}\text { Extra radiation dose, } 2 \mathrm{D} \text { images, bones are used as a } \\
\text { surrogate for the prostate position. } \\
\text { With FM: invasive, risk of infection/bleeding, migra- } \\
\text { tion, presence of FM can perturb dose }{ }^{158-161} \text {. }\end{array}$ \\
\hline $\mathrm{CT} \pm \mathrm{FM}$ & $\begin{array}{l}3 D \text { images, easy to } \\
\text { acquire, FM easily } \\
\text { visualized, operator } \\
\text { independent }\end{array}$ & $\begin{array}{l}\text { Extra radiation dose, not commonly available in } \\
\text { treatment room, moderate contrast without FM. } \\
\text { With FM: invasive, risk of infection/bleeding, migra- } \\
\text { tion, FMs cannot define prostate fully in } 3 D \text {, presence } \\
\text { of FM can perturb dose }{ }^{158-161} \text {. }\end{array}$ \\
\hline $\mathrm{CBCT} \pm \mathrm{FM}$ & $\begin{array}{l}3 \mathrm{D} \text { images, easy to } \\
\text { acquire, FM easily } \\
\text { visualized, operator } \\
\text { independent }\end{array}$ & $\begin{array}{l}\text { Extra radiation dose, moderate contrast without FM. } \\
\text { With FM: see previous line ( } C T \pm F M) \text {. }\end{array}$ \\
\hline US & $\begin{array}{l}\text { No extra radiation } \\
\text { dose, high imaging } \\
\text { contrast, real-time } \\
\text { tracking }\end{array}$ & $\begin{array}{l}\text { Image acquisition uses patient contact and is user } \\
\text { dependent, requires good training, not all systems } \\
\text { are } 3 D \text {, small FOV. }\end{array}$ \\
\hline MRI & $\begin{array}{l}\text { No extra radiation } \\
\text { dose, high tissue } \\
\text { contrast }\end{array}$ & Complex, not available in treatment room yet. \\
\hline
\end{tabular}

The prostate is relatively easy to image with US in the presence of a full bladder, which acts as an acoustic window. Several groups have reported on their findings on the implementation of US IGRT systems (table 5).

\section{Issues for US imaging for prostate RT}

Several publications comparing 2DUS and FM-based $x$-ray imaging prostate localization methods have raised concerns regarding the accuracy of US-based techniques ${ }^{33,42,156}$. The main concerns are US user variability and abdominal probe pressure causing organ motion. However, in some cases the prostate displacement attributed to probe pressure, might actually be the SOS artefact described earlier. Excessive pressure on the patient's skin is, in particular in radiology, believed to be necessary to acquire high quality images. Some studies, however, have reported that only a small amount of pressure is sufficient 
for good image quality for RT applications ${ }^{131,157}$. By matching the TX and SIM US images, which are affected similarly by the probe pressure, the target displacement will be reduced (the same holds for the SOS error). It remains a problem if treatment planning based on US SIM images is attempted. For intermodality systems where a TX US image is compared to a SIM CT image a target shift may be incorrectly affected by probe pressure. Measures to reduce the probe pressure artefact were discussed in the previous section.

Table 5: Studies on the clinical implementation and utility of US IGRT systems.

\begin{tabular}{|c|c|c|}
\hline Focus & Study Facts & Findings \\
\hline $\begin{array}{l}\text { First clinical } \\
\text { experience }\end{array}$ & $\begin{array}{l}\text { \# patients: 4-54; } \\
\text { 185-4698 daily scans }\end{array}$ & $\begin{array}{l}\text { Daily prostate movement can be }>1 \mathrm{~cm} \text {. Transabdominal } \\
\text { US-based stereotactic guidance is a safe method for } \\
\text { correcting patient positioning (BAT system: }{ }^{165,166} \text {, SonArray } \\
\text { system: }{ }^{167} \text { ). Dosimetric evaluation studies show a better } \\
\text { dose coverage when US IGRT shift implemented (BAT } \\
\text { system: }{ }^{168,169} \text {, Clarity system: }{ }^{170} \text { ). In one study }{ }^{171} \text {, the } \\
\text { degradation of the treatment plan using US IGRT was } \\
\text { higher compared to MVCT. } \\
\text { Practical issues: modest increase in treatment setup time } \\
166 \text {. Adequate bladder filling and modest probe pressure } \\
\text { were crucial to achieve adequate image quality without } \\
\text { systematic prostate displacements }{ }^{157} \text {. }\end{array}$ \\
\hline $\begin{array}{l}\text { Analysis of } \\
\text { acute toxicity }\end{array}$ & $\begin{array}{l}\text { \# patients: } 22-50 ; \\
\text { BAT system }\end{array}$ & $\begin{array}{l}\text { Rate of acute gastrointestinal toxicity reduced with the use } \\
\text { of US IGRT. No change in acute genitourinary toxicity rate } \\
\text { was found }{ }^{172} \text {. Without US IGRT significant more acute and } \\
\text { a trend towards more late rectal toxicity was found } \\
\text { Daily adaptive US IGRT reduces rectal toxicity in the case of } \\
\text { empty rectum and to minimize geometric miss of prostate } \\
174\end{array}$ \\
\hline $\begin{array}{l}\text { Reports of } \\
\text { experience, } \\
\text { based on large } \\
\text { patient groups }\end{array}$ & $\begin{array}{l}\text { \# patients: } 130-387 \\
\text { \# scans: } 3509-10327\end{array}$ & $\begin{array}{l}\text { Image quality of } 95 \% \text { of daily US images with BAT was } \\
\text { acceptable for patient positioning. In } 3 \% \text { of the cases, } \\
\text { major alignment errors remained }{ }^{141} \text {. Particularly large } \\
\text { patients or those who cannot maintain bladder filling, } \\
\text { transabdominal US images may not show enough anatomi- } \\
\text { cal detail to allow accurate localization. User training in US } \\
\text { scanning and interpretation is needed }{ }^{130} \text {. }\end{array}$ \\
\hline
\end{tabular}

One-to-one comparisons of US with other IGRT techniques are shown in table 6 . The first few studies in the table $e^{128,134,162,163}$ compare US IGRT to skin marks or bone alignment on portal images, which do not represent the prostate. The differences found were reported as prostate motion, and US IGRT was considered to be an improvement for patient positioning. However, Serago et al. ${ }^{128}$ expressed reservations about the technique. They stress the prerequisite of good US image quality and address that US IGRT is not suitable for a subset of patients with physical characteristics that make good quality images difficult to obtain, e.g. patients with high body mass index or those who cannot maintain a full bladder. 


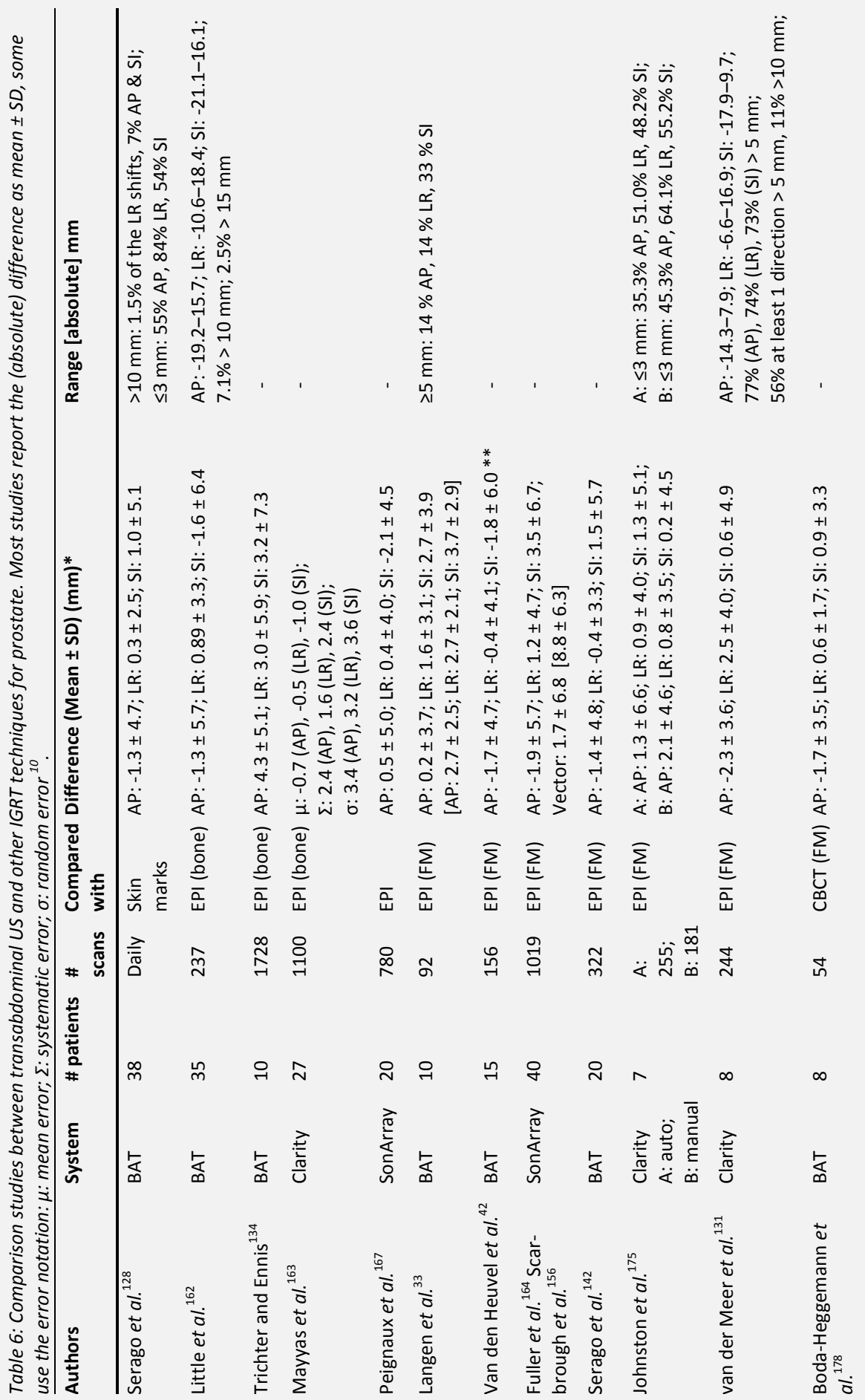




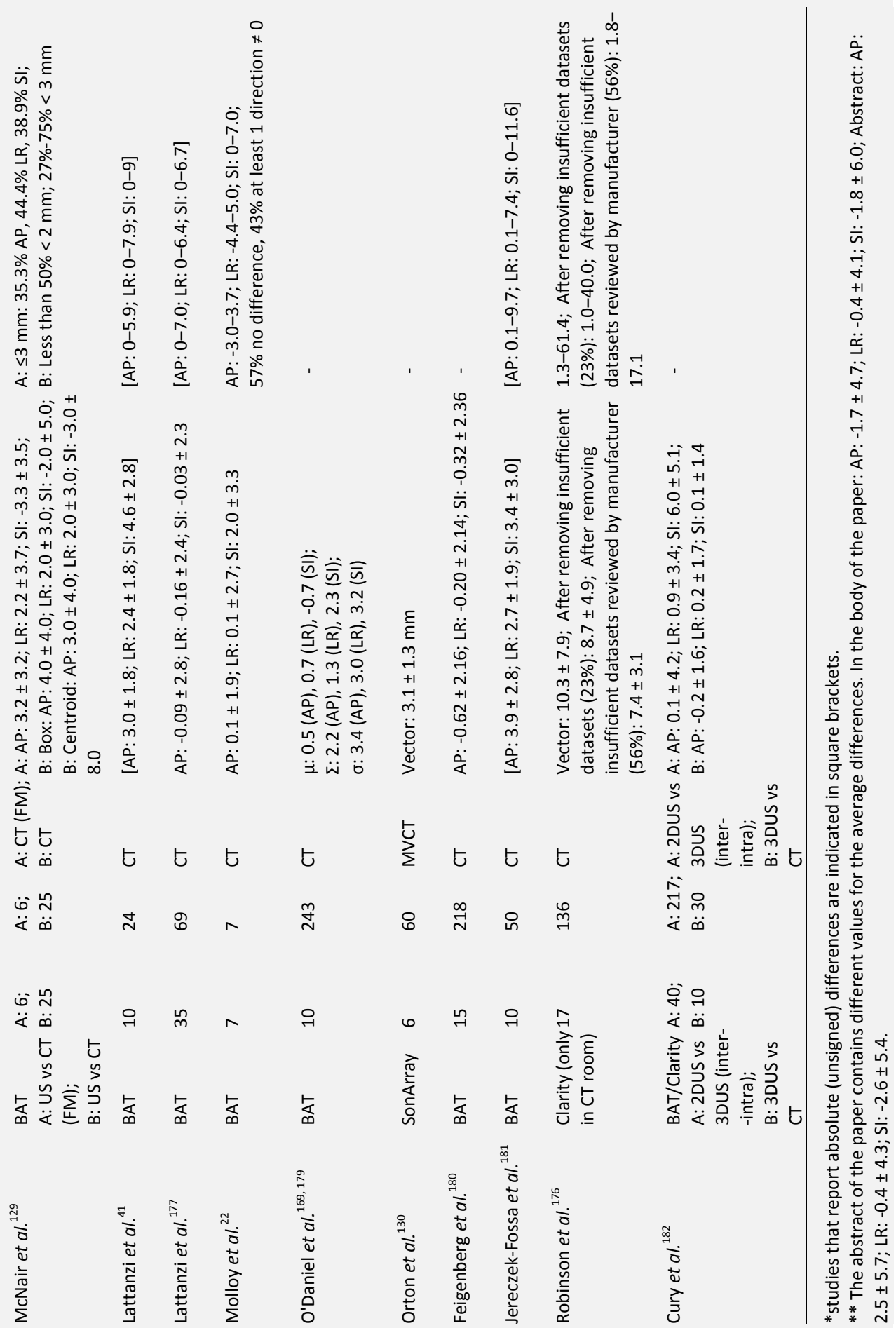


Comparisons of US with CT+FM gave conflicting conclusions, even though the reported differences were small. In the absence of a 'real' gold standard and without taking into account the estimation of the uncertainties of both systems, the decision of which system performs better is subjective. One can only say whether IGRT systems are interchangeable or not, and clinical judgment must dictate whether the difference is of importance ${ }^{131,164}$.

Discrepancies between a reference modality and US led to unfavorable conclusions towards US in some studies ${ }^{42,142,175,176}$ even though these discrepancies may be due to uncertainties in the reference modality. For instance in some studies ${ }^{42,131,156,164}$ the CT images were acquired less than a week after FM implantation, which makes it probable that the FM were not yet fully anchored. Also larger US displacements sometimes resulted in negative conclusions since this resulted in undesirable larger PTV margins. In these studies, overestimation of the prostate motion with US was assumed to be more probable than underestimation with FM. Studies comparing US to EPI and/or CBCT with a linear regression analysis ${ }^{41,131,162,163,165,177}$ found these techniques to be comparable (table $6, R$ $=0.44-0.88(\mathrm{AP}), 0.24-0.91(\mathrm{LR}), 0.12-0.87(\mathrm{SI}))$. Clinical workflow and the patient's preference towards non-invasive procedures may also guide the choice between the different methods.

\section{Breast}

Several clinical studies have shown that adjuvant RT post lumpectomy of localized breast cancer leads to improved survival rates ${ }^{183}$. The use of a boost field given to tissue surrounding the surgical cavity following whole breast photon irradiation has been shown to improve treatment outcome significantly ${ }^{184,185}$. This tissue, close to the edge of the surgical cavity may contain viable cancer cells, which may remain after surgical excision, and is therefore deemed to be the radiation target. Boost radiation is often delivered by external electron beams, or in more recent clinical practice, by photon beams. Over time, the target may change in size, shape and position due to patient setup, breast edema, tissue remodeling in the cavity, changes in post-operative breast anatomy, and respiratory motion. To ensure accurate delivery of the boost radiation some form of breast IGRT is needed. To reduce the incidence and severity of toxicity, to reduce the probability of secondary radiation-induced tumors and also for cosmetic reasons the amount of normal tissue irradiated to the boost dose should be kept as low as possible.

\section{Breast imaging modalities in $R T$}

Imaging modalities that have been used to define the surgical cavity for RT are planar xray imaging with $\mathrm{kV}$ or MV photons, CT or CBCT imaging, MRI and US imaging. Several studies used metallic FM, often surgical clips, or in the case of MV imaging, gold FM, implanted during lumpectomy to aid the indirect visualization of the target area. Using CT and $\mathrm{CBCT}$ imaging it is possible to visualize seroma, the surrounding tissue and FM. Using kV planar imaging, only metallic markers are visible. In US imaging only the seroma cavity (i.e. liquid filled) and the surrounding tissue are visible. All the imaging modalities mentioned above make use of surrogates to identify the target region. Imaging modalities which rely on surrogates such as the chest wall or the skin to obtain the position of the 
cavity are considered inappropriate due to the geometric uncertainties in the position of the target relative to bony anatomy and breast surfaces ${ }^{192}$. Table 7 gives an overview of the advantages and disadvantages of the various imaging modalities used for breast boost RT. In older studies, planar x-ray imaging with implanted FM was mostly used, which evolved into CT or CBCT imaging, with or without FM. The use of FM as an IGRT 'gold standard' has been questioned by many due to imaging artefacts, FM migration and inadequate 3D coverage (only a limited number of points within the cavity can be visualized, typically less than 10$)^{8,186,187,193}$.

Table 7. Comparison of breast imaging modalities (all non-invasive, if FM are implanted during lumpectomy).

\begin{tabular}{|c|c|c|}
\hline Modality & Advantages & Disadvantages \\
\hline $\begin{array}{l}\text { kV or MV } \\
\text { x-ray + FM }\end{array}$ & $\begin{array}{l}\text { Easy to acquire, good } \\
\text { visibility of FM }\end{array}$ & $\begin{array}{l}\text { 2D images, extra radiation dose, FM migration, metal } \\
\text { imaging artefacts, FM reliability to determine cavity } \\
\text { depends on surgical implant practice and delineation } \\
\text { practice. }\end{array}$ \\
\hline $\mathrm{CT} \pm \mathrm{FM}$ & $\begin{array}{l}\text { 3D images, with FM some- } \\
\text { times perceived as gold } \\
\text { standard }\end{array}$ & $\begin{array}{l}\text { Extra radiation dose, moderate contrast of seroma } \\
\text { cavity, not commonly available in treatment room, use } \\
\text { of original planning CT for boost planning may be } \\
\text { inadequate due to cavity changes }{ }^{186} \text {. } \\
\text { With FM: FM migration, FM cannot define cavity fully } \\
\text { in 3D, FM reliability to determine cavity depends on } \\
\text { surgical implant practice and delineation practice. }\end{array}$ \\
\hline US & $\begin{array}{l}\text { No extra radiation dose, } \\
\text { high imaging contrast of } \\
\text { seroma cavity, can easily } \\
\text { be used in treatment room }\end{array}$ & $\begin{array}{l}\text { Cavity more difficult to see with time post-surgery, } \\
\text { cavity visibility lower for smaller breast and young } \\
\text { patients ( }<40 \text { yrs) } 187 \text {, scanning may distort breast } \\
\text { geometry. }\end{array}$ \\
\hline $\mathrm{CBCT} \pm \mathrm{FM}$ & $\begin{array}{l}\text { Can easily be used in } \\
\text { treatment room, markers } \\
\text { can be seen easily }\end{array}$ & $\begin{array}{l}\text { Possibly poor cavity visualization depending on } \\
\text { imaging artefacts }{ }^{188} \text {, extra radiation dose, problematic } \\
\text { conversion of Hounsfield units to electron densities for } \\
\text { dose calculations }{ }^{189} \text {. } \\
\text { Collision risk of CBCT unit with patient due to off-axis } \\
\text { setup for breast irradiation }{ }^{190} \text {. }\end{array}$ \\
\hline MRI & High tissue contrast & $\begin{array}{l}\text { Not available in treatment room, doesn't seem to add } \\
\text { much to } \mathrm{CT}^{191} \text {. }\end{array}$ \\
\hline
\end{tabular}

The first studies to investigate 2DUS imaging for breast RT appeared in the late 90s and early 2000s ${ }^{187,194-196}$. These studies were concerned with electron boost except one which explored US imaging for breast brachytherapy ${ }^{194}$. These early reports established the usefulness of US imaging for breast RT, but also pointed out that US cavity volumes can differ significantly from CT derived volumes, with the latter nearly always exceeding the former.

In 2007 the first study ${ }^{193}$ reporting the use of 3DUS imaging for breast RT established that this technique was a considerable improvement over 2DUS imaging. They attributed this to the dependence of distance measurements in 2DUS on the probe angle on the breast surface. The cavity was visible on 3DUS in $83 \%$ of 40 patients. They noted that all 
imaging modalities (2DUS, 3DUS and CT) correlated poorly with specimen volume due to cavity changes after surgery. These authors were also the first to report US to CT registration using a camera to track the US probe motion with IR emitters. This technique had been reported earlier for prostate US imaging. The 3DUS system employed was a Diasus (Dynamic Imaging, Livingston, Scotland, UK). They used a dedicated US camera-tracked pointer to point towards the CT FM to register US-CT. They developed a highly original phantom (olives with stones replaced by vaseline, embedded in gelatin to mimic a lesion). US phantoms are generally more difficult to manufacture than CT phantoms due to phantom materials such as plastics being less tissue equivalent for US wave propagation than for $x$-ray attenuation.

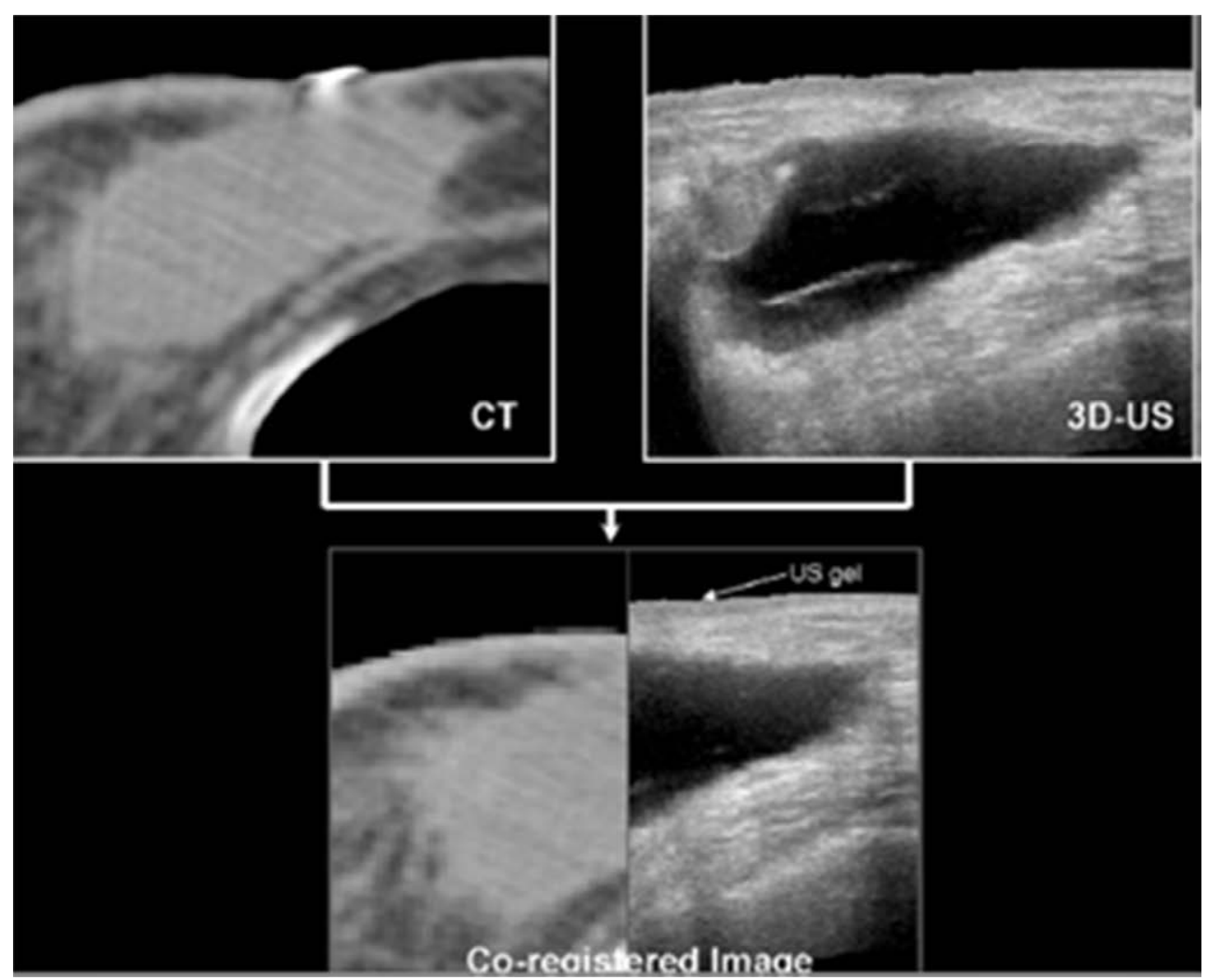

Figure 5: CT image of breast seroma cavity with the cavity indicated in light grey (top left) and US image of the non-echogenic cavity visible in dark grey (top right). Bottom: co-registered image (the mismatch at the skin is attributed to the US gel layer on top of the breast). Adapted from Berrang et $a l^{8}$, with kind permission.

A similar 3DUS study ${ }^{8}$ for accelerated partial breast irradiation (APBI) with photon beams used the Restitu system (now Clarity: Elekta, Stockholm, Sweden). They also registered 3DUS and CT images and didn't use FM. Figure 5 from this work compares the seroma cavity as seen on CT and US images. They obtained a 3DUS-CT co-registration that was accurate to within $2 \mathrm{~mm}$ or less in 19/20 patients. They reported that CT seroma visibility 
was reduced with dense breast parenchyma, smaller seroma volume, and smaller volume of excised breast tissue, while US seroma clarity was not affected by these factors. They also noted that US imaging had improved inter-observer consistency compared with CT in $8 / 20$ cases.

Surprisingly, until 2010 no breast RT studies had been reported that also investigated the consequences of target localization on the radiation dose to the target. The first study $^{197}$ to do so investigated dose variations due to inter-fraction breast motion for electron boost, using the Clarity 3DUS imaging system. They found significant changes in dose between different fractions; the mean dose to the CTV in the combined dose distribution (from 3DUS imaging) deviated from that of the reference scenario where a CT image dose calculation is performed only at the start of the boost treatments from $60 \%$ less to $23 \%$ greater. Compared to the dose calculation based on the initial CT planning, even larger differences could be obtained.

Another study focused on the workflow for 3DUS cavity volume determination in breast boost RT both with electron and photon beams ${ }^{198}$. They used the Clarity system with its semi-automatic cavity delineation tools, and the Ultrapath feature which shows on screen the US scan path from the reference US scan to increase reproducibility of subsequent US scans. For electron boost the Clarity system for breast IGRT has a digitizer, which is a camera-tracked pointer tool with IR markers that can be used to digitize the surgical scar. This then provides an external anatomy landmark, in conjunction with the internal seroma cavity landmark, in the beam's eye view. The aforementioned 3DUS breast studies clearly indicate the usefulness of 3DUS imaging as an adjoint to CT imaging, with sometimes clear advantages over CT.

Another imaging modality that has been explored for breast $\mathrm{RT}$ is $\mathrm{CBCT}^{188-190,199-201}$. In many cases CBCT imaging is used to match chest wall and skin surface, due to the poor visibility of the cavity. These images suffer more from scatter artefacts than CT images, and therefore image quality is reduced and conversion of Hounsfield units to electron density, needed for dose calculation, is compromised. Many reports also express concern about the extra radiation dose incurred by CBCT imaging for multiple treatment fractions. From several studies it is clear that CBCT imaging, which has been shown to be very useful for other treatment sites, is sub-optimal for breast ${ }^{188,190,199,200}$. CBCT imaging and 3DUS imaging could probably be used as complementary modalities, but this needs more study.

The use of MRI to identify the target area for breast RT has been reported but did not add much improvement to CT-defined targets ${ }^{191}$. Implantable markers for US imaging exist, but to our knowledge have not been reported yet in the literature for use in breast RT. CEUS imaging to distinguish diagnostically malignant from benign breast tissue has been described recently ${ }^{202}$ but has not yet been explored to aid breast RT, as far as we know.

Issues for US imaging for breast RT

All studies cited use linear array US transducers in the range of 5-12 $\mathrm{MHz}$. Breast distortion due to US probe pressure is an issue but many studies report that the effect is minimal if scanning is done with minimal probe pressure and using a high viscosity gel ${ }^{8,186,197,198}$. 
One study mentioned an algorithm to correct US images for pressure ${ }^{119,137}$. 2DUS appears sufficient for surgical cavity imaging for simple boost fields but 3DUS is preferred for complex conformal photon boost studies or $\mathrm{APB}^{8,193}$. In many older studies of US versus CT only cavity visibility, or dimensions (e.g. distal cavity wall depth from skin surface) were compared, whereas the more recent publications use image registration ${ }^{8,186,197,198}$. SOS correction for breast was explored recently ${ }^{113}$, but was not adopted in any of the studies discussed here.

There are several factors that influence the US visibility of the cavity. Breast size has no influence according to some ${ }^{196}$ (2DUS study), while others found that smaller breasts decreased visibility ${ }^{187}$ (2DUS study). Some studies reported that the US visibility didn't depend on time elapsed since surgery, but most studies agree that time degrades the image quality, possibly due to resolution of seroma. Menopausal status doesn't seem to have an influence ${ }^{187}$. US appears to work best for patients with low body fat ${ }^{196}$ and older patients ( $>40$ years) ${ }^{187}$. The effect of motion caused by breathing on US imaging seems an ill-studied phenomenon.

Several studies confirm that the cavity appears smaller in US than in CT images. The CT image can include not only the fluid-filled cavity and the surrounding walls, but also the postoperative architectural distortion, breast tissue stranding, fibrosis, and normal dense breast parenchyma ${ }^{8}$. The US cavity volume was also reported to reduce at a higher rate over time than the CT volume ${ }^{186}$. They found that the tumor bed volume shrunk on average by $10 \%$ on CT and $26 \%$ on US between scans done during initial planning and just before electron boost. The explanation was that as the seroma reduces over time, fibrotic tissue takes its place and CT cannot distinguish the electron densities of fibrotic tissue and seroma liquid, whereas US can distinguish the difference between solid tissue and liquids. Some studies reported the US cavity to appear more shallow ${ }^{187}$ which may indicate SOS aberration $^{113}$.

\section{Other sites}

Visibility of abdominal lesions (e.g. liver) is usually quite poor with CT based systems without using contrast media. US IGRT has been used for other organs than prostate and breast, but reports have been few. A 3DUS system was designed for extracranial stereotactic guidance ${ }^{203}$, which later led to the commercial SonArray system (ZMed, Inc, Ashland, MA, USA; later this system was acquired by Varian Medical Systems, Palo Alto, CA, USA), but it is now no longer marketed. They achieved a spatial accuracy of $1.5 \mathrm{~mm}$, which is vital for stereotactic treatments. In 2004 the utility of the BAT system was investigated for 62 patients with upper abdominal tumors ${ }^{81}$ and it was successfully implemented in the clinical routine. In only $4.2 \%$ of the BAT alignments the quality of the US images was not sufficient to visualize the target, mainly due to colonic and gastric air.

The potential of US guidance with the 2DUS BAT device in RT of the gallbladder has been reported ${ }^{204}$. By correction for inter-fraction motion of up to $5 \mathrm{~cm}$ (mean: $1.4 \mathrm{~cm}$ ) the treatment margins could be reduced to a level to compensate for breathing intra-fraction motion. The authors proposed dose escalation trials, based on their confidence in US guidance. The 2DUS BAT system was also used in a study on IGRT for pancreas cancer ${ }^{205}$. Vector shifts exceeding 1 and $2 \mathrm{~cm}$ were needed in 50\% and 13.5\%, respectively, of the 
targeting attempts. In several patients, observed systematic US shifts led to CT rescanning and replanning. The large measured shifts support the use of generous margins (up to 3 $\mathrm{cm}$ ) for pancreatic RT in the absence of image guidance. These data indicate that image guidance for every fraction may lead to more conformal treatments with significant normal tissue sparing and potential dose escalation to the tumor.

An US IGRT BAT procedure was combined with active breathing control (ABC) for stereotactic liver treatment in one patient ${ }^{206}$. Later they evaluated the upper abdominal US IGRT BAT procedure (without breathing control) in a comparison study against $\mathrm{CBCT}^{207}$. Three out of the 15 patients were excluded due to unsatisfactory image quality for the US images. In 7 patients the quality of the US images was good; in the other 5 patients the quality was satisfactory. For the good quality US images the average differences ( \pm standard deviation (SD)) between US and CBCT IGRT were $(-0.1 \pm 3.11) \mathrm{mm}$ in the left-right direction $(\mathrm{LR}),(0.93 \pm 4.31) \mathrm{mm}$ in the superior-inferior direction $(\mathrm{SI})$, and $(0.71 \pm 3.15)$ $\mathrm{mm}$ in the anterior-posterior direction (AP). For patients with satisfactory image quality, the differences were $(-0.6 \pm 5.26) \mathrm{mm} \mathrm{LR},(1.76 \pm 4.92) \mathrm{mm} \mathrm{SI}$, and $(1.19 \pm 4.75) \mathrm{mm} \mathrm{AP}$.

The user variability in US IGRT for liver patients was investigated ${ }^{133}$. The uncertainties of US scanning and US image segmentation in liver lesions were determined with and without the use of $A B C$. In free breathing, the combined mean uncertainties of repeated scan uncertainty and inter- and intra-observer variation were $1.3 \mathrm{~mm} \mathrm{LR}, 3.7 \mathrm{~mm} \mathrm{SI}$, and $1.8 \mathrm{~mm}$ AP. Using $A B C$ the mean uncertainty reduced by $1.7 \mathrm{~mm}$ in the SI direction. For the $L R$ and AP directions, no difference was observed.

A 2DUS study of 20 bladder cancer patients ${ }^{208}$ measured post-void bladder volumes before and after RT as a surrogate for bladder motion. Bladder dimensions were acquired in 2 orthogonal planes from which the volume was derived. A significant change in voided bladder volume throughout the treatment course occurred in several patients. The mean inter-fraction volume of five patients was $50 \%$ larger than the reference US volume assessed at time of CT scanning and planning. Systematic intra-fraction volume increase of bladder can be expected at a commonly assumed rate of $\sim 1 \mathrm{~cm}^{3} \mathrm{~min}^{-1}$. This study confirmed a measurable intra-fraction increase in bladder volume in $89 \%$ of cases, with results varying highly among patients. Potential infringements on bladder treatment margins and/or over-irradiation of normal tissue were frequently seen in this work. It would appear that for bladder RT daily image guidance is essential, but this would benefit from a 3DUS technique. In two somewhat related publications, 3DUS full bladder measurements were employed to assess motion of the cervix ${ }^{209}$ or the rectum ${ }^{210}$ during RT. Ahmad et al used the US-volumes for finding a correlation between bladder filling changes and cervix-uterus displacements. By measuring the bladder filling with US and the correlation with cervix position a best plan of the day could be selected from the patient-specific plan library ${ }^{209,211-213}$. It would appear that despite claims made in these papers, the US devices used did not result in displayed 3DUS images, but only reported certain bladder measurements. All studies discussed in this section used convex array US transducers, as far as we can discern.

The use of US IGRT for the direct imaging and positioning of gynecological sites is in development, but not many studies have been published yet. The use of 2DUS was successfully introduced for cervix brachytherapy ${ }^{214}$, and recently a phantom and a patient study from the same group were reported with a 3DUS-system for external $\mathrm{RT}^{215,216}$. 
US imaging has been reported in head and neck RT, typically employing linear array transducers operating around $10 \mathrm{MHz}$ (superficial imaging). It has been used for e.g. assessing radiation-induced xerostomia (dry mouth) by quantifying tissue texture ${ }^{217}$, and for imaging the radiation effects on pediatric thyroid glands ${ }^{218}$. See also $\mathrm{O}^{\prime}$ Shea et $a{ }^{1}{ }^{1}$. The only 3DUS head \& neck study that we are aware of was published as conference proceedings $^{219}$ and attempted to quantify the temporal volumetric evolution of cervical lymph nodes throughout a course of RT, with the aim of aiding adaptive RT.

Endobronchial US imaging in lung cancer has received much attention in diagnostic imaging, e.g. for staging lung cancer ${ }^{220}$, but as far as we know, no direct applications in RT have been reported. There are no accounts known to us on the use of US imaging in brain RT. The latter two applications are difficult for US imaging due to the limited penetration power of US waves in these anatomical regions.

\section{FUTURE DEVELOPMENTS}

From this review it is clear that US imaging is a mature imaging technology, which is available for image guidance applications in RT. The reality is, however, that far fewer RT centers use US imaging than $x$-ray based imaging (EPID, CT, CBCT) for verification of treatment setup. The only clinical RT application that has known modest success is daily US imaging of prostate, for setup corrections. This was the most popular application in the late 90 s and it remains so until today. X-ray based imaging has been more popular, and is used by most advanced RT centers for regular (but usually not quite daily) verification of the patient anatomy for various target organs. The advantages and disadvantages of both imaging techniques have been discussed in this review. There are currently high expectations for another form of image guidance: MR imaging, especially when integrated with a $\operatorname{linac}^{143}$. This technique could offer high imaging contrast during therapy, but is not without its disadvantages of which the high cost will certainly be one. The imaging modalities discussed here are to some extent complementary. Therefore, one could envisage future RT departments having access to multiple forms of imaging for treatment guidance, which could be used in combination depending on the application.

Currently, it is not easy to predict the future of US imaging in RT. US image quality has improved significantly recently. Several novel applications of US to RT will be discussed extensively in the companion review paper ${ }^{1}$. These techniques show that US imaging also has the potential to provide advanced functional imaging over $\mathrm{x}$-ray based imaging. Most verification imaging currently done in RT is for inter-fraction motion. For intra-fraction motion, US imaging can play an important role. It can be used to quantify motion of organs or endogenous structures in 3D and in real time, without the need for implanted markers. X-ray based imaging will usually be too slow and/or need invasive FMs to achieve the same goal. However, until now widespread US use in RT has been hampered due to the extensive training requirements. Therefore there is an urgent need to make US imaging user-independent and automatic. Some recent advances have been reported in this direction by using robotic operatorless systems ${ }^{31}$. These first efforts may lead to novel concepts, such as adhesive US probes with phased crystal arrays, obviating the need for 
probe positioning systems and mechanically swept beams as in current applications (such as Autoscan). These could even be left on the patient during beam delivery, enabling true intra-fraction motion management, and eventually providing direct feedback to the irradiation system.

Much work is also being done to segment structures automatically in real time, and one can envisage applications such as real-time dose display based on anatomical 3D deformation fields derived from daily US imaging. Real-time display of the 3D differences between the planned and the delivered dose will then also be within reach. These new developments, in combination with automated operator-less imaging may lead to a much higher clinical acceptance of the US IGRT technique in RT.

The current applications of IGRT in particle beam therapy are limited, but with the growing popularity of this form of therapy the need for image guidance in this field will also increase. US imaging may have a new opportunity to be integrated with the particle beamline to enable real-time organ monitoring. Challenges such as interfacing real-time US equipment with scanning particle beams will have to be addressed. Lastly, US imaging offers some exciting opportunities such as real-time in vivo particle beam range monitoring, based on thermo-acoustic processes in tissue ${ }^{221,222}$.

\section{ACKNOWLEDGMENTS}

We thank Dr Martin Lachaine for carefully reviewing the manuscript. Dr Philip Evans, Dr Tony Falco and Rui Lopes are thanked for many useful discussions. SvdM is funded by GROW (School for Oncology and Developmental Biology, Maastricht University). JCB, EJH and ToS acknowledge Cancer Research UK for funding.

\section{REFERENCES}

1 T. O'Shea, J. Bamber, D. Fontanarosa, et al., "Review of Ultrasound Image Guidance in External Beam Radiotherapy, Part 2: intra-fraction motion management and novel applications.", 2015 Phys Med Biol (submitted))

2 P.M. Evans, "Anatomical imaging for radiotherapy", 2008 Phys Med Biol (53:R151-191)

3 J. Bradley, W.L. Thorstad, S. Mutic, et al., "Impact of FDG-PET on radiation therapy volume delineation in non-small-cell lung cancer", 2004 Int J Radiat Oncol Biol Phys (59:78-86)

4 P. Lind, I. Igerc, T. Beyer, et al., "Advantages and limitations of FDG PET in the follow-up of breast cancer", 2004 Eur J Nucl Med Mol Imaging (31 Suppl 1:S125-134)

5 D.L. Schwartz, E. Ford, J. Rajendran, et al., "FDG-PET/CT imaging for preradiotherapy staging of head-andneck squamous cell carcinoma", 2005 Int J Radiat Oncol Biol Phys (61:129-136)

6 G.S. Payne, M.O. Leach, "Applications of magnetic resonance spectroscopy in radiotherapy treatment planning", 2006 Br J Radiol (79 Spec No 1:S16-26)

7 D. Flores-Tapia, G. Thomas, N. Venugopal, et al., presented at the Proc. IEEE Eng Med Biol Soc, Vancouver, BC, 2008 (unpublished).

8 T.S. Berrang, P.T. Truong, C. Popescu, et al., "3D ultrasound can contribute to planning CT to define the target for partial breast radiotherapy", 2009 Int J Radiat Oncol Biol Phys (73:375-383)

9 E.D. Yorke, P. Keall, F. Verhaegen, "Anniversary Paper: Role of medical physicists and the AAPM in improving geometric aspects of treatment accuracy and precision", 2008 Med Phys (35:828)

10 M. van Herk, "Errors and margins in radiotherapy", 2004 Semin Radiat Oncol (14:52-64)

11 A. Bel, P.H. Vos, P.T. Rodrigus, et al., "High-precision prostate cancer irradiation by clinical application of an offline patient setup verification procedure, using portal imaging", 1996 Int J Radiat Oncol Biol Phys (35:321-332) 
12 J.E. Schewe, J.M. Balter, K.L. Lam, R.K. ten Haken, "Measurement of patient setup errors using port films and a computer-aided graphical alignment tool", 1996 Med Dosim (21:97-104)

13 U.A. van der Heide, A.N. Kotte, H. Dehnad, et al., "Analysis of fiducial marker-based position verification in the external beam radiotherapy of patients with prostate cancer", 2007 Radiother Oncol (82:38-45)

14 M. Oldham, D. Letourneau, L. Watt, et al., "Cone-beam-CT guided radiation therapy: A model for on-line application", 2005 Radiother Oncol (75:271-278)

15 R.D. Foster, T.D. Solberg, H.S. Li, et al., "Comparison of transabdominal ultrasound and electromagnetic transponders for prostate localization", $2010 \mathrm{~J}$ Appl Clin Med Phys (11:2924)

16 B.W. Raaymakers, J.J. Lagendijk, J. Overweg, et al., "Integrating a 1.5 T MRI scanner with a 6 MV accelerator: proof of concept", 2009 Phys Med Biol (54:N229-237)

17 A.Y. Fung, K.M. Ayyangar, D. Djajaputra, et al., "Ultrasound-based guidance of intensity-modulated radiation therapy", 2006 Med Dosim (31:20-29)

18 D.A. Kuban, L. Dong, R. Cheung, et al., "Ultrasound-based localization", 2005 Semin Radiat Oncol (15:180191)

19 A. Fenster, D.B. Downey, H.N. Cardinal, "Three-dimensional ultrasound imaging", 2001 Phys Med Biol (46:R67-99)

20 R.J. Rose, S. Allwin, "Computerized Cancer Detection and Classification Using Ultrasound Images: A Survey", 2013 International Journal of Engineering Research and Development (5:36-47)

21 M.H. Fuchsjager, A.G. Maier, W. Schima, et al., "Comparison of transrectal sonography and double-contrast MR imaging when staging rectal cancer", 2003 AJR Am J Roentgenol (181:421-427)

22 J.A. Molloy, S. Srivastava, B.F. Schneider, "A method to compare supra-pubic ultrasound and CT images of the prostate: technique and early clinical results", 2004 Med Phys (31:433-442)

23 Y.L. Huang, Y.R. Jiang, D.R. Chen, W.K. Moon, "Level set contouring for breast tumor in sonography", $2007 \mathrm{~J}$ Digit Imaging (20:238-247)

24 W.L. Smith, C. Lewis, G. Bauman, et al., "Prostate volume contouring: a 3D analysis of segmentation using 3DTRUS, CT, and MR", 2007 Int J Radiat Oncol Biol Phys (67:1238-1247)

25 D. Batchelar, M. Gaztanaga, M. Schmid, et al., "Validation study of ultrasound-based high-dose-rate prostate brachytherapy planning compared with CT-based planning", 2014 Brachytherapy (13:75-79)

26 X.J. Juan-Senabre, J. Lopez-Tarjuelo, A. Conde-Moreno, et al., "Uncertainties and CTV to PTV margins quantitative assessment using cone-beam CT technique in clinical application for prostate, and head and neck irradiation tumors", 2011 Clin Transl Oncol (13:819-825)

27 E.J. Harris, E.M. Donovan, C.E. Coles, et al., "How does imaging frequency and soft tissue motion affect the PTV margin size in partial breast and boost radiotherapy?", 2012 Radiother Oncol (103:166-171)

28 E. Mace, G. Montaldo, I. Cohen, et al., "Functional ultrasound imaging of the brain", 2011 Nat Methods (8:662-664)

29 H.P. Dietz, "Ultrasound imaging of the pelvic floor. Part II: three-dimensional or volume imaging", 2004 Ultrasound Obstet Gynecol (23:615-625)

30 M. Majida, I.H. Braekken, W. Umek, et al., "Interobserver repeatability of three- and four-dimensional transperineal ultrasound assessment of pelvic floor muscle anatomy and function", 2009 Ultrasound Obstet Gynecol (33:567-573)

31 M. Lachaine, T. Falco, "Intrafractional prostate motion management with the Clarity autoscan system", 2013 Medical Physics International (1:72-80)

32 C. Enke, K. Ayyangar, C.B. Saw, et al., "Inter-observer variation in prostate localization utilizing BAT", 2002 Int J Radiat Oncol Biol Phys (54:269)

33 K.M. Langen, J. Pouliot, C. Anezinos, et al., "Evaluation of ultrasound-based prostate localization for imageguided radiotherapy", 2003 Int J Radiat Oncol Biol Phys (57:635-644)

34 D. Yan, E. Ziaja, D. Jaffray, et al., "The use of adaptive radiation therapy to reduce setup error: a prospective clinical study", 1998 Int J Radiat Oncol Biol Phys (41:715-720)

35 X. Artignan, M.H. Smitsmans, J.V. Lebesque, et al., "Online ultrasound image guidance for radiotherapy of prostate cancer: impact of image acquisition on prostate displacement", 2004 Int J Radiat Oncol Biol Phys (59:595-601)

36 D. Fontanarosa, S. van der Meer, E. Harris, F. Verhaegen, "A CT based correction method for speed of sound aberration for ultrasound based image guided radiotherapy", 2011 Med Phys (38:2665-2673)

37 B. Block, "Color Atlas of Ultrasound Anatomy", (Thieme, Stuttgart, Germany, 2012). 
38 T. Liu, M.M. Mansukhani, M.C. Benson, et al., "A feasibility study of novel ultrasonic tissue characterization for prostate-cancer diagnosis: 2D spectrum analysis of in vivo data with histology as gold standard", 2009 Med Phys (36:3504-3511)

39 D.J. Brascho, "Tumor localization and treatment planning with ultrasound", 1977 Cancer (39:697-705)

40 M. Uematsu, T. Fukui, A. Shioda, et al., "A dual computed tomography linear accelerator unit for stereotactic radiation therapy: a new approach without cranially fixated stereotactic frames", 1996 Int J Radiat Oncol Biol Phys (35:587-592)

41 J. Lattanzi, S. McNeeley, W. Pinover, et al., "A comparison of daily CT localization to a daily ultrasoundbased system in prostate cancer", 1999 Int J Radiat Oncol Biol Phys (43:719-725)

42 F. van den Heuvel, T. Powell, E. Seppi, et al., "Independent verification of ultrasound based image-guided radiation treatment, using electronic portal imaging and implanted gold markers", 2003 Med Phys (30:2878-2887)

43 M.C. Abramowitz, E. Bossart, R. Flook, et al., "Noninvasive Real-time Prostate Tracking Using a Transperineal Ultrasound Approach", 2012 Int J Radiat Oncol Biol Phys (84:S133)

$44 \mathrm{H}$. Wallace, J. Archambault, M. Lachaine, "Autoscan transperineal ultasound of the pelvis for prostate gland localization. ", 2012 Int J Radiat Oncol Biol Phys (84:S373)

45 B. Salter, M. Szegedi, P. Rassiah-Szegedi, et al., "Presentation of a new intrafractional prostate monitoring method with ultrasound image guidance during radiotherapy treatment. ", 2013 Med Phys (40:375)

46 R. Sandhu, O. Marina, S. Martin, et al., "Real-time 4d ultrasound prostate gland motion tracking during radiotherapy fraction delivery.", 2013 Med Phys (40:415)

47 C. Lee, K.M. Langen, W. Lu, et al., "Assessment of parotid gland dose changes during head and neck cancer radiotherapy using daily megavoltage computed tomography and deformable image registration", 2008 Int J Radiat Oncol Biol Phys (71:1563-1571)

48 J.T. Bushberg, The essential physics of medical imaging, 3rd ed. (Wolters Kluwer Health/Lippincott Williams \& Wilkins, Philadelphia, 2012).

49 P.L. Carson, "Biomedical ultrasonics. By P.N.T. Wells, Ph.D., Academic Press, Inc. London, Copyright 1977. 635 pages.", $1978 \mathrm{~J}$ Clin Ultrasound (6:126-127)

50 J.J. Wild, "The use of ultrasonic pulses for the measurement of biological tissues and the detection of tissue density changes", 1950 Surgery (27:183-199)

51 L.L. Beranek, A.s.o. America, Acoustics, [2nd ed.] ed. (American institute of physics for the Acoustical society of America, [S. L.], 1990).

52 F.A. Duck, A.C. Baker, H.C. Starritt, Ultrasound in medicine. (Institute of Physics Pub., Bristol ; Philadelphia, Pa., 1998).

53 S. Webb, Webb's Physics of Medical Imaging, Second Edition ed. (CRC Press, Taylor \& Francis Group, Boca Raton, FL, 2012).

54 J.C. Bamber, N. Miller, M. Tristam, "Diagnostic ultrasound", in Webb's Physics of Medical Imaging, edited by M.A. Flower (CRC Press Taylor and Francis Group, Boca Raton, 2012), pp. 351-486.

55 C.R. Hill, F. Dunn, "Physical Principles of Medical Ultrasound edited by C. R. Hill", 1987 The Journal of the Acoustical Society of America (82:721)

56 C. Hill, J. Bamber, G. Ter Haar, Physical Principles of Medical Ultrasonics, 2nd Edition. (Wiley, 2004).

57 P.N.T. Wells, Biomedical Ultrasonics. (Academic, London, 1977).

58 A. Fenster, G. Parraga, J. Bax, "Three-dimensional ultrasound scanning", 2011 Interface Focus (1:503-519)

59 A. Fenster, D.B. Downey, "Three-dimensional ultrasound imaging and its use in quantifying organ and pathology volumes", 2003 Anal Bioanal Chem (377:982-989)

60 J.F. Brinkley, W.D. McCallum, S.K. Muramatsu, D.Y. Liu, "Fetal weight estimation from ultrasonic threedimensional head and trunk reconstructions: evaluation in vitro", 1982 Am J Obstet Gynecol (144:715-721)

61 J.F. Greenleaf, M. Belohlavek, T.C. Gerber, et al., "Multidimensional visualization in echocardiography: an introduction", 1993 Mayo Clin Proc (68:213-220)

62 R.N. Rankin, A. Fenster, D.B. Downey, et al., "Three-dimensional sonographic reconstruction: techniques and diagnostic applications", 1993 AJR Am J Roentgenol (161:695-702)

63 W.L. Smith, A. Fenster, "Optimum scan spacing for three-dimensional ultrasound by speckle statistics", 2000 Ultrasound Med Biol (26:551-562)

64 N. Pagoulatos, D.R. Haynor, Y. Kim, "A fast calibration method for 3-D tracking of ultrasound images using a spatial localizer", 2001 Ultrasound Med Biol (27:1219-1229)

65 E.A. Geiser, L.G. Christie, Jr., D.A. Conetta, et al., "A mechanical arm for spatial registration of twodimensional echocardiographic sections", 1982 Cathet Cardiovasc Diagn (8:89-101) 
66 J.F. Brinkley, W.D. McCallum, S.K. Muramatsu, D.Y. Liu, "Fetal weight estimation from lengths and volumes found by three-dimensional ultrasonic measurements", $1984 \mathrm{~J}$ Ultrasound Med (3:163-168)

67 P.R. Detmer, G. Bashein, T. Hodges, et al., "3D ultrasonic image feature localization based on magnetic scanhead tracking: in vitro calibration and validation", 1994 Ultrasound Med Biol (20:923-936)

68 R.J. Housden, A.H. Gee, G.M. Treece, R.W. Prager, "Sensorless reconstruction of unconstrained freehand 3D ultrasound data", 2007 Ultrasound Med Biol (33:408-419)

69 G. Stetten, R. Tamburo, "Real-time three-dimensional ultrasound methods for shape analysis and visualization", 2001 Methods (25:221-230)

70 J. Deng, "Terminology of three-dimensional and four-dimensional ultrasound imaging of the fetal heart and other moving body parts", 2003 Ultrasound Obstet Gynecol (22:336-344)

71 T.R. Nelson, D.H. Pretorius, M. Sklansky, S. Hagen-Ansert, "Three-dimensional echocardiographic evaluation of fetal heart anatomy and function: acquisition, analysis, and display", $1996 \mathrm{~J}$ Ultrasound Med (15:1-9 quiz 11-12)

72 W. Lee, "3D fetal ultrasonography", 2003 Clin Obstet Gynecol (46:850-867)

73 J. Deng, C.H. Rodeck, "Current applications of fetal cardiac imaging technology", 2006 Curr Opin Obstet Gynecol (18:177-184)

74 J.C. Bamber, R.J. Eckersley, P. Hubregtse, et al., "Data-Processing for 3-D Ultrasound Visualization of Tumor Anatomy and Blood-Flow", in Visualization in Biomedical Computing, 1992, Vol. 1808 (SPIE, 1992), pp. 651663.

75 A. Fenster, K. Surry, W. Smith, et al., "3D ultrasound imaging: applications in image-guided therapy and biopsy", 2002 Computers \& Graphics (26:557-568)

76 T.D. Mast, "Empirical relationships between acoustic parameters in human soft tissues", 2000 Acoustics Research Letters Online (1:37-42)

77 J.C. Bamber, "Speed of sound", in Physical Principles of Medical Ultrasonics, 2nd Edition, edited by C.R. Hill, J.C. Bamber, G.R. ter Haar (John Wiley, Chichester, 2004), pp. 167-190.

78 L.G. Bouchet, S.L. Meeks, G. Goodchild, et al., "Calibration of three-dimensional ultrasound images for image-guided radiation therapy", 2001 Phys Med Biol (46:559-577)

79 T.C. Poon, R.N. Rohling, "Comparison of calibration methods for spatial tracking of a 3-D ultrasound probe", 2005 Ultrasound Med Biol (31:1095-1108)

80 J. Schlosser, C. Kirmizibayrak, V. Shamdasani, et al., "Automatic 3D ultrasound calibration for image guided therapy using intramodality image registration", 2013 Phys Med Biol (58:7481-7496)

81 M. Fuss, B.J. Salter, S.X. Cavanaugh, et al., "Daily ultrasound-based image-guided targeting for radiotherapy of upper abdominal malignancies", 2004 Int J Radiat Oncol Biol Phys (59:1245-1256)

82 W. Wein, B. Roper, N. Navab, "Integrating diagnostic B-mode ultrasonography into CT-based radiation treatment planning", 2007 IEEE Trans Med Imaging (26:866-879)

83 S. Ghose, A. Oliver, R. Marti, et al., "A survey of prostate segmentation methodologies in ultrasound, magnetic resonance and computed tomography images", 2012 Comput Methods Programs Biomed (108:262-287)

84 D. Shen, Y. Zhan, C. Davatzikos, "Segmentation of prostate boundaries from ultrasound images using statistical shape model", 2003 IEEE Trans Med Imaging (22:539-551)

85 A. Jendoubi, Z. Jianchao, M.F. Chouikha, presented at the Proc. International Symposium on Information Theory, 2004. ISIT 2004. 2004 (unpublished).

86 N. Betrouni, M. Vermandel, D. Pasquier, et al., "Segmentation of abdominal ultrasound images of the prostate using a priori information and an adapted noise filter", 2005 Comput Med Imaging Graph (29:4351)

87 M. Ding, B. Chiu, I. Gyacskov, et al., "Fast prostate segmentation in 3D TRUS images based on continuity constraint using an autoregressive model", 2007 Med Phys (34:4109-4125)

88 M.P. Kuenen, T.A. Saidov, H. Wijkstra, et al., "Spatiotemporal correlation of ultrasound contrast agent dilution curves for angiogenesis localization by dispersion imaging", 2013 IEEE Trans Ultrason Ferroelectr Freq Control (60:2665-2669)

89 A. Sadeghi-Naini, O. Falou, H. Tadayyon, et al., "Conventional frequency ultrasonic biomarkers of cancer treatment response in vivo", 2013 Transl Oncol (6:234-243)

90 H. Kim, E. Brander, M.S. Huq, S. Beriwal, "Clinical Application of Ultrasound Imaging in Radiation Therapy", in Ultrasound Imaging - Medical Applications, edited by O. Minin ((InTech, 2011).

91 B.C. Porter, D.J. Rubens, J.G. Strang, et al., "Three-dimensional registration and fusion of ultrasound and MRI using major vessels as fiducial markers", 2001 IEEE Trans Med Imaging (20:354-359) 
92 J.M. Blackall, G.P. Penney, A.P. King, D.J. Hawkes, "Alignment of sparse freehand 3-D ultrasound with preoperative images of the liver using models of respiratory motion and deformation", 2005 IEEE Trans Med Imaging (24:1405-1416)

93 T. Mizowaki, G.N. Cohen, A.Y. Fung, M. Zaider, "Towards integrating functional imaging in the treatment of prostate cancer with radiation: the registration of the MR spectroscopy imaging to ultrasound/CT images and its implementation in treatment planning", 2002 Int J Radiat Oncol Biol Phys (54:1558-1564)

94 C. Reynier, J. Troccaz, P. Fourneret, et al., "MRI/TRUS data fusion for prostate brachytherapy. Preliminary results", 2004 Med Phys (31:1568-1575)

95 R. Szeliski, S. Lavallée, "Matching 3-D anatomical surfaces with non-rigid deformations using octreesplines", 1996 Int J Comput Vision (18:171-186)

96 S. Ourselin, A. Roche, S. Prima, N. Ayache, "Block Matching: A General Framework to Improve Robustness of Rigid Registration of Medical Images", in Medical Image Computing and Computer-Assisted Intervention - MICCAI 2000, Vol. 1935, edited by S. Delp, A. DiGoia, B. Jaramaz (Springer Berlin Heidelberg, 2000), pp. 557-566.

97 V. Daanen, J. Gastaldo, J.Y. Giraud, et al., "MRI/TRUS data fusion for brachytherapy", 2006 Int J Med Robot (2:256-261)

98 V.V. Karnik, A. Fenster, J. Bax, et al., "Assessment of image registration accuracy in three-dimensional transrectal ultrasound guided prostate biopsy", 2010 Med Phys (37:802-813)

99 D. Shen, E.H. Herskovits, C. Davatzikos, "An adaptive-focus statistical shape model for segmentation and shape modeling of 3-D brain structures", 2001 IEEE Trans Med Imaging (20:257-270)

100 C. Davatzikos, "Spatial transformation and registration of brain images using elastically deformable models", 1997 Comput Vis Image Underst (66:207-222)

101 R. Narayanan, J. Kurhanewicz, K. Shinohara, et al., presented at the IEEE International Symposium on Biomedical Imaging: From Nano to Macro, 2009. ISBI '09. 2009 (unpublished).

102 Y. Hu, H.U. Ahmed, C. Allen, et al., "MR to ultrasound image registration for guiding prostate biopsy and interventions", 2009 Medical image computing and computer-assisted intervention : MICCAI ... International Conference on Medical Image Computing and Computer-Assisted Intervention (12:787-794)

103 Y. Hu, H.U. Ahmed, Z. Taylor, et al., "MR to ultrasound registration for image-guided prostate interventions", 2012 Med Image Anal (16:687-703)

104 J. Moorrees, E. Bezak, "Four dimensional CT imaging: a review of current technologies and modalities", 2012 Australas Phys Eng Sci Med (35:9-23)

105 J.A. Molloy, S.A. Oldham, "Benchmarking a novel ultrasound-CT fusion system for respiratory motion management in radiotherapy: assessment of spatio-temporal characteristics and comparison to 4DCT", 2008 Med Phys (35:291-300)

106 O. Ukimura, I.S. Gill, "Image-fusion, augmented reality, and predictive surgical navigation", 2009 Urol Clin North $\operatorname{Am}(36: 115-123$, vii)

107 A. Stang, H. Keles, S. Hentschke, et al., "Real-time ultrasonography-computed tomography fusion imaging for staging of hepatic metastatic involvement in patients with colorectal cancer: initial results from comparison to US seeing separate CT images and to multidetector-row CT alone", 2010 Invest Radiol (45:491-501)

108 S. Nakano, M. Yoshida, K. Fujii, et al., "Real-time virtual sonography, a coordinated sonography and MRI system that uses magnetic navigation, improves the sonographic identification of enhancing lesions on breast MRI", 2012 Ultrasound in medicine \& biology (38:42-49)

109 H.X. Xu, M.D. Lu, L.N. Liu, L.H. Guo, "Magnetic navigation in ultrasound-guided interventional radiology procedures", 2012 Clin Radiol (67:447-454)

110 V. Narayana, P.L. Roberson, A.T. Pu, et al., "Impact of differences in ultrasound and computed tomography volumes on treatment planning of permanent prostate implants", 1997 Int J Radiat Oncol Biol Phys (37:1181-1185)

111 S.C. Hoffelt, L.M. Marshall, M. Garzotto, et al., "A comparison of CT scan to transrectal ultrasoundmeasured prostate volume in untreated prostate cancer", 2003 Int J Radiat Oncol Biol Phys (57:29-32)

112 B.J. Salter, B. Wang, M.W. Szegedi, et al., "Evaluation of alignment error due to a speed artifact in stereotactic ultrasound image guidance", 2008 Phys Med Biol (53:N437-445)

113 D. Fontanarosa, S. van der Meer, E. Bloemen-van Gurp, et al., "Magnitude of speed of sound aberration corrections for ultrasound image guided radiotherapy for prostate and other anatomical sites", 2012 Med Phys (39:5286-5292)

114 D. Fontanarosa, S. van der Meer, F. Verhaegen, "On the significance of density-induced speed of sound variations on US-guided radiotherapy", 2012 Med Phys (39:6316-6323) 
115 D. Fontanarosa, S. Pesente, F. Pascoli, et al., "A speed of sound aberration correction algorithm for curvilinear ultrasound transducers in ultrasound-based image-guided radiotherapy", 2013 Phys Med Biol (58:13411360)

116 J.A. Molloy, G. Chan, A. Markovic, et al., "Quality assurance of U.S.-guided external beam radiotherapy for prostate cancer: report of AAPM Task Group 154", 2011 Med Phys (38:857-871)

117 E.J. Harris, N.R. Miller, J.C. Bamber, et al., "Performance of ultrasound based measurement of 3D displacement using a curvilinear probe for organ motion tracking", 2007 Phys Med Biol (52:5683-5703)

118 M. Wang, R. Rohling, C. Duzenli, et al., "Evaluation of targeting errors in ultrasound-assisted radiotherapy", 2008 Ultrasound Med Biol (34:1944-1956)

119 E.J. Harris, R. Symonds-Taylor, G.M. Treece, et al., "Evaluation of a three-dimensional ultrasound localisation system incorporating probe pressure correction for use in partial breast irradiation", $2009 \mathrm{Br} \mathrm{J}$ Radiol (82:839-846)

120 J. Schlosser, K. Salisbury, D. Hristov, "Telerobotic system concept for real-time soft-tissue imaging during radiotherapy beam delivery", 2010 Med Phys (37:6357-6367)

121 J. Schlosser, K. Salisbury, D. Hristov, "Online image-based monitoring of soft-tissue displacements for radiation therapy of the prostate", 2012 Int J Radiat Oncol Biol Phys (83:1633-1640)

122 M. Kaar, M. Figl, R. Hoffmann, et al., "Automatic patient alignment system using 3D ultrasound", 2013 Med Phys (40:041714)

123 W.A. Tome, S.L. Meeks, N.P. Orton, et al., "Commissioning and quality assurance of an optically guided three-dimensional ultrasound target localization system for radiotherapy", 2002 Med Phys (29:1781-1788)

124 L.A. Drever, M. Hilts, "Daily quality assurance phantom for ultrasound image guided radiation therapy", 2007 J Appl Clin Med Phys (8:2467)

125 W.A. Tome, N.P. Orton, "Quality assurance of ultrasound imaging systems for target localization and online setup corrections", 2008 Int J Radiat Oncol Biol Phys (71:S53-56)

126 M. Fuss, S.X. Cavanaugh, C. Fuss, et al., "Daily stereotactic ultrasound prostate targeting: inter-user variability", 2003 Technol Cancer Res Treat (2:161-170)

127 C. Fiandra, A. Guarneri, F. Munoz, et al., "Impact of the observers' experience on daily prostate localization accuracy in ultrasound-based IGRT with the Clarity platform", $2014 \mathrm{~J}$ Appl Clin Med Phys (15:4795)

128 C.F. Serago, S.J. Chungbin, S.J. Buskirk, et al., "Initial experience with ultrasound localization for positioning prostate cancer patients for external beam radiotherapy", 2002 Int J Radiat Oncol Biol Phys (53:1130-1138)

129 H.A. McNair, S.A. Mangar, J. Coffey, et al., "A comparison of CT- and ultrasound-based imaging to localize the prostate for external beam radiotherapy", 2006 Int J Radiat Oncol Biol Phys (65:678-687)

130 N.P. Orton, H.A. Jaradat, W.A. Tome, "Clinical assessment of three-dimensional ultrasound prostate localization for external beam radiotherapy", 2006 Med Phys (33:4710-4717)

131 S. van der Meer, E. Bloemen-van Gurp, J. Hermans, et al., "Critical assessment of intramodality 3D ultrasound imaging for prostate IGRT compared to fiducial markers", 2013 Med Phys (40:071707)

132 A. Landry, T. Berrang, I. Gagne, et al., "Investigation of variability in image acquisition and contouring during 3D ultrasound guidance for partial breast irradiation", 2014 Radiation Oncology (9:35)

133 E. Bloemen-van Gurp, S. van der Meer, J. Hendry, et al., "Active breathing control in combination with ultrasound imaging: a feasibility study of image guidance in stereotactic body radiation therapy of liver lesions", 2013 Int J Radiat Oncol Biol Phys (85:1096-1102)

134 F. Trichter, R.D. Ennis, "Prostate localization using transabdominal ultrasound imaging", 2003 Int J Radiat Oncol Biol Phys (56:1225-1233)

135 B. Dobler, S. Mai, C. Ross, et al., "Evaluation of possible prostate displacement induced by pressure applied during transabdominal ultrasound image acquisition", 2006 Strahlenther Onkol (182:240-246)

136 M. Fargier-Voiron, B. Presles, P. Pommier, et al., "Impact of probe pressure variability on prostate localization for ultrasound-based image-guided radiotherapy", 2014 Radiother Oncol)

137 G.M. Treece, R.W. Prager, A.H. Gee, L. Berman, "Correction of probe pressure artifacts in freehand 3D ultrasound", 2002 Med Image Anal (6:199-214)

138 G.M. Treece, A.H. Gee, R.W. Prager, "RF and amplitude-based probe pressure correction for 3D ultrasound", 2005 Ultrasound Med Biol (31:493-503)

139 M.A. Lediju Bell, H.T. Sen, I. Iordachita, et al., presented at the Proc. SPIE 9036, Medical Imaging 2014: Image-Guided Procedures, Robotic Interventions, and Modeling, San Diego, California, USA, 2014 (unpublished). 
140 D.S. Mohan, P.A. Kupelian, T.R. Willoughby, "Short-course intensity-modulated radiotherapy for localized prostate cancer with daily transabdominal ultrasound localization of the prostate gland", 2000 Int J Radiat Oncol Biol Phys (46:575-580)

141 A. Chandra, L. Dong, E. Huang, et al., "Experience of ultrasound-based daily prostate localization", 2003 Int J Radiat Oncol Biol Phys (56:436-447)

142 C.F. Serago, S.J. Buskirk, T.C. Igel, et al., "Comparison of daily megavoltage electronic portal imaging or kilovoltage imaging with marker seeds to ultrasound imaging or skin marks for prostate localization and treatment positioning in patients with prostate cancer", 2006 Int J Radiat Oncol Biol Phys (65:1585-1592)

143 J.J. Lagendijk, B.W. Raaymakers, A.J. Raaijmakers, et al., "MRI/linac integration", 2008 Radiother Oncol $(86: 25-29)$

144 Y. Hu, O.L. Green, Y. Feng, et al., "Image Performance Characterization of an MRI-Guided Radiation Therapy System", 2013 International journal of radiation oncology, biology, physics (87:S13)

145 D.A. Jaffray, M. Carlone, S. Breen, et al., "Development of a Novel Platform for MR-Guided Radiation Therapy", 2013 International journal of radiation oncology, biology, physics (87:S13)

146 K. Knight, N. Touma, L. Zhu, et al., "Implementation of daily image-guided radiation therapy using an inroom CT scanner for prostate cancer isocentre localization", 2009 Journal of Medical Imaging and Radiation Oncology (53:132-138)

147 A.R. Padhani, V.S. Khoo, J. Suckling, et al., "Evaluating the effect of rectal distension and rectal movement on prostate gland position using cine MRI", 1999 Int J Radiat Oncol Biol Phys (44:525-533)

148 D. Mah, G. Freedman, B. Milestone, et al., "Measurement of intrafractional prostate motion using magnetic resonance imaging", 2002 Int J Radiat Oncol Biol Phys (54:568-575)

149 A.Y. Fung, C.A. Enke, K.M. Ayyangar, et al., "Prostate motion and isocenter adjustment from ultrasoundbased localization during delivery of radiation therapy", 2005 Int J Radiat Oncol Biol Phys (61:984-992)

150 O. Gayou, M. Miften, "Comparison of mega-voltage cone-beam computed tomography prostate localization with online ultrasound and fiducial markers methods", 2008 Med Phys (35:531-538)

151 M.E. Poli, W. Parker, H. Patrocinio, et al., "An assessment of PTV margin definitions for patients undergoing conformal 3D external beam radiation therapy for prostate cancer based on an analysis of 10,327 pretreatment daily ultrasound localizations", 2007 Int J Radiat Oncol Biol Phys (67:1430-1437)

152 A.M. Henry, C. Wilkinson, J.P. Wylie, et al., "Trans-perineal implantation of radio-opaque treatment verification markers into the prostate: an assessment of procedure related morbidity, patient acceptability and accuracy", 2004 Radiother Oncol (73:57-59)

153 J.F. Langenhuijsen, E.N. van Lin, L.A. Kiemeney, et al., "Ultrasound-guided transrectal implantation of gold markers for prostate localization during external beam radiotherapy: complication rate and risk factors", 2007 Int J Radiat Oncol Biol Phys (69:671-676)

154 S. Igdem, H. Akpinar, G. Alco, et al., "Implantation of fiducial markers for image guidance in prostate radiotherapy: patient-reported toxicity", $2009 \mathrm{Br}$ J Radiol (82:941-945)

155 V. Fonteyne, P. Ost, G. Villeirs, et al., "Improving positioning in high-dose radiotherapy for prostate cancer: safety and visibility of frequently used gold fiducial markers", 2012 Int J Radiat Oncol Biol Phys (83:46-52)

156 T.J. Scarbrough, N.M. Golden, J.Y. Ting, et al., "Comparison of ultrasound and implanted seed marker prostate localization methods: Implications for image-guided radiotherapy", 2006 Int J Radiat Oncol Biol Phys (65:378-387)

157 M. Pinkawa, M. Pursch-Lee, B. Asadpour, et al., "Image-guided radiotherapy for prostate cancer. Implementation of ultrasound-based prostate localization for the analysis of inter- and intrafraction organ motion", 2008 Strahlenther Onkol (184:679-685)

158 G. Landry, B. Reniers, L. Lutgens, et al., "Dose reduction in LDR brachytherapy by implanted prostate gold fiducial markers", 2012 Med Phys (39:1410-1417)

159 J. Cheung, R.J. Kudchadker, X.R. Zhu, et al., "Dose perturbations and image artifacts caused by carboncoated ceramic and stainless steel fiducials used in proton therapy for prostate cancer", 2010 Phys Med Biol (55:7135-7147)

160 C.T. Huang, C.Y. Chen, C.C. Ho, C.J. Yu, "A rare constellation of empyema, lung abscess, and mediastinal abscess as a complication of endobronchial ultrasound-guided transbronchial needle aspiration", 2011 Eur J Cardiothorac Surg (40:264-265)

161 J.C. Chow, G.N. Grigorov, "Monte Carlo simulations of dose near a nonradioactive gold seed", 2006 Med Phys (33:4614-4621) 
162 D.J. Little, L. Dong, L.B. Levy, et al., "Use of portal images and BAT ultrasonography to measure setup error and organ motion for prostate IMRT: implications for treatment margins", 2003 Int J Radiat Oncol Biol Phys (56:1218-1224)

163 E. Mayyas, I.J. Chetty, M. Chetvertkov, et al., "Evaluation of multiple image-based modalities for imageguided radiation therapy (IGRT) of prostate carcinoma: A prospective study", 2013 Med Phys (40:041707)

164 C.D. Fuller, C.R. Thomas, S. Schwartz, et al., "Method comparison of ultrasound and kilovoltage x-ray fiducial marker imaging for prostate radiotherapy targeting", 2006 Phys Med Biol (51:4981-4993)

165 J. Lattanzi, S. McNeeley, S. Donnelly, et al., "Ultrasound-based stereotactic guidance in prostate cancer-quantification of organ motion and set-up errors in external beam radiation therapy", 2000 Comput Aided Surg (5:289-295)

166 J. Morr, T. DiPetrillo, J.S. Tsai, et al., "Implementation and utility of a daily ultrasound-based localization system with intensity-modulated radiotherapy for prostate cancer", 2002 Int J Radiat Oncol Biol Phys (53:1124-1129)

167 K. Peignaux, G. Truc, I. Barillot, et al., "Clinical assessment of the use of the Sonarray system for daily prostate localization", 2006 Radiother Oncol (81:176-178)

168 T. Falco, G. Shenouda, C. Kaufmann, et al., "Ultrasound imaging for external-beam prostate treatment setup and dosimetric verification", 2002 Med Dosim (27:271-273)

169 J.C. O'Daniel, L. Dong, L. Zhang, et al., "Dosimetric comparison of four target alignment methods for prostate cancer radiotherapy", 2006 Int J Radiat Oncol Biol Phys (66:883-891)

170 D.J. Fraser, Y. Chen, E. Poon, et al., "Dosimetric consequences of misalignment and realignment in prostate 3DCRT using intramodality ultrasound image guidance", 2010 Med Phys (37:2787-2795)

171 C. Peng, K. Kainz, C. Lawton, X.A. Li, "A comparison of daily megavoltage CT and ultrasound image guided radiation therapy for prostate cancer", 2008 Med Phys (35:5619-5628)

172 A.B. Jani, J. Gratzle, E. Muresan, et al., "Analysis of acute toxicity with use of transabdominal ultrasonography for prostate positioning during intensity-modulated radiotherapy", 2005 Urology (65:504-508)

173 M. Bohrer, P. Schroder, G. Welzel, et al., "Reduced rectal toxicity with ultrasound-based image guided radiotherapy using BAT (B-mode acquisition and targeting system) for prostate cancer", 2008 Strahlenther Onkol (184:674-678)

174 N.M. Reddy, D. Nori, W. Sartin, et al., "Influence of volumes of prostate, rectum, and bladder on treatment planning CT on interfraction prostate shifts during ultrasound image-guided IMRT", 2009 Med Phys (36:5604-5611)

175 H. Johnston, M. Hilts, W. Beckham, E. Berthelet, "3D ultrasound for prostate localization in radiation therapy: a comparison with implanted fiducial markers", 2008 Med Phys (35:2403-2413)

176 D. Robinson, D. Liu, S. Steciw, et al., "An evaluation of the Clarity 3D ultrasound system for prostate localization", 2012 J App/ Clin Med Phys (13:3753)

177 J. Lattanzi, S. McNeeley, A. Hanlon, et al., "Ultrasound-based stereotactic guidance of precision conformal external beam radiation therapy in clinically localized prostate cancer", 2000 Urology (55:73-78)

178 J. Boda-Heggemann, F.M. Kohler, B. Kupper, et al., "Accuracy of ultrasound-based (BAT) prostaterepositioning: a three-dimensional on-line fiducial-based assessment with cone-beam computed tomography", 2008 Int J Radiat Oncol Biol Phys (70:1247-1255)

179 J.C. O'Daniel, L. Dong, L. Zhang, et al., "Daily bone alignment with limited repeat CT correction rivals daily ultrasound alignment for prostate radiotherapy", 2008 Int J Radiat Oncol Biol Phys (71:274-280)

180 S.J. Feigenberg, K. Paskalev, S. McNeeley, et al., "Comparing computed tomography localization with daily ultrasound during image-guided radiation therapy for the treatment of prostate cancer: a prospective evaluation", 2007 J Appl Clin Med Phys (8:2268)

181 B.A. Jereczek-Fossa, F. Cattani, C. Garibaldi, et al., "Transabdominal ultrasonography, computed tomography and electronic portal imaging for 3-dimensional conformal radiotherapy for prostate cancer", 2007 Strahlenther Onkol (183:610-616)

182 F.L. Cury, G. Shenouda, L. Souhami, et al., "Ultrasound-based image guided radiotherapy for prostate cancer: comparison of cross-modality and intramodality methods for daily localization during external beam radiotherapy", 2006 Int J Radiat Oncol Biol Phys (66:1562-1567)

183 M. Clarke, R. Collins, S. Darby, et al., "Effects of radiotherapy and of differences in the extent of surgery for early breast cancer on local recurrence and 15-year survival: an overview of the randomised trials", 2005 Lancet (366:2087-2106)

184 N. Antonini, H. Jones, J.C. Horiot, et al., "Effect of age and radiation dose on local control after breast conserving treatment: EORTC trial 22881-10882", 2007 Radiother Oncol (82:265-271) 
185 H. Bartelink, J.C. Horiot, P.M. Poortmans, et al., "Impact of a higher radiation dose on local control and survival in breast-conserving therapy of early breast cancer: 10-year results of the randomized boost versus no boost EORTC 22881-10882 trial", 2007 J Clin Oncol (25:3259-3265)

186 P. Wong, T. Muanza, E. Reynard, et al., "Use of three-dimensional ultrasound in the detection of breast tumor bed displacement during radiotherapy", 2011 Int J Radiat Oncol Biol Phys (79:39-45)

187 A. Warszawski, R. Baumann, J.H. Karstens, "Sonographic guidance for electron boost planning after breastconserving surgery", $2004 \mathrm{~J}$ Clin Ultrasound (32:333-337)

188 E.A. White, J. Cho, K.A. Vallis, et al., "Cone beam computed tomography guidance for setup of patients receiving accelerated partial breast irradiation", 2007 Int J Radiat Oncol Biol Phys (68:547-554)

189 J. Wang, W. Hu, G. Cai, et al., "Using corrected cone-beam CT image for accelerated partial breast irradiation treatment dose verification: the preliminary experience", 2013 Radiat Oncol (8:214)

190 T. Fatunase, Z. Wang, S. Yoo, et al., "Assessment of the residual error in soft tissue setup in patients undergoing partial breast irradiation: results of a prospective study using cone-beam computed tomography", 2008 Int J Radiat Oncol Biol Phys (70:1025-1034)

191 A.M. Kirby, J.R. Yarnold, P.M. Evans, et al., "Tumor bed delineation for partial breast and breast boost radiotherapy planned in the prone position: what does MRI add to X-ray CT localization of titanium clips placed in the excision cavity wall?", 2009 Int J Radiat Oncol Biol Phys (74:1276-1282)

192 R. Topolnjak, C. van Vliet-Vroegindeweij, J.J. Sonke, et al., "Breast-conserving therapy: radiotherapy margins for breast tumor bed boost", 2008 Int J Radiat Oncol Biol Phys (72:941-948)

193 C.E. Coles, C.J. Cash, G.M. Treece, et al., "High definition three-dimensional ultrasound to localise the tumor bed: a breast radiotherapy planning study", 2007 Radiother Oncol (84:233-241)

194 D.A. DeBiose, E.M. Horwitz, A.A. Martinez, et al., "The use of ultrasonography in the localization of the lumpectomy cavity for interstitial brachytherapy of the breast", 1997 Int J Radiat Oncol Biol Phys (38:755759)

195 M.C. Smitt, R.L. Birdwell, D.R. Goffinet, "Breast electron boost planning: comparison of CT and US", 2001 Radiology (219:203-206)

196 J. Ringash, T. Whelan, E. Elliott, et al., "Accuracy of ultrasound in localization of breast boost field", 2004 Radiother Oncol (72:61-66)

197 D.J. Fraser, P. Wong, K. Sultanem, F. Verhaegen, "Dosimetric evolution of the breast electron boost target using 3D ultrasound imaging", 2010 Radiother Oncol (96:185-191)

198 M. Chadha, A. Young, C. Geraghty, et al., "Image guidance using 3D-ultrasound (3D-US) for daily positioning of lumpectomy cavity for boost irradiation", 2011 Radiat Oncol (6:45)

199 P. Jain, T. Marchant, M. Green, et al., "Inter-fraction motion and dosimetric consequences during breast intensity-modulated radiotherapy (IMRT)", 2009 Radiother Oncol (90:93-98)

200 G. Jozsef, J.K. DeWyngaert, S.J. Becker, et al., "Prospective study of cone-beam computed tomography image-guided radiotherapy for prone accelerated partial breast irradiation", 2011 Int J Radiat Oncol Biol Phys (81:568-574)

201 E.M. Donovan, I. Castellano, S. Eagle, E. Harris, "Clinical implementation of kilovoltage cone beam CT for the verification of sequential and integrated photon boost treatments for breast cancer patients", $2012 \mathrm{Br} J \mathrm{Ra}$ diol (85:e1051-1057)

202 Z. Yuan, J. Quan, Z. Yunxiao, et al., "Diagnostic value of contrast-enhanced ultrasound parametric imaging in breast tumors", 2013 J Breast Cancer (16:208-213)

203 S.L. Meeks, J.M. Buatti, L.G. Bouchet, et al., "Ultrasound-guided extracranial radiosurgery: technique and application", 2003 Int J Radiat Oncol Biol Phys (55:1092-1101)

204 C.D. Fuller, C.R. Thomas, Jr., A. Wong, et al., "Image-guided intensity-modulated radiation therapy for gallbladder carcinoma", 2006 Radiother Oncol (81:65-72)

205 M. Fuss, A. Wong, C.D. Fuller, et al., "Image-guided intensity-modulated radiotherapy for pancreatic carcinoma", 2007 Gastrointest Cancer Res (1:2-11)

206 J. Boda-Heggemann, C. Walter, S. Mai, et al., "Frameless stereotactic radiosurgery of a solitary liver metastasis using active breathing control and stereotactic ultrasound", 2006 Strahlenther Onkol (182:216221)

207 J. Boda-Heggemann, P. Mennemeyer, H. Wertz, et al., "Accuracy of ultrasound-based image guidance for daily positioning of the upper abdomen: an online comparison with cone beam CT", 2009 Int J Radiat Oncol Biol Phys (74:892-897) 
208 C.A. McBain, M.M. Green, J. Stratford, et al., "Ultrasound imaging to assess inter- and intra-fraction motion during bladder radiotherapy and its potential as a verification tool", 2009 Clin Oncol (R Coll Radiol) (21:385393)

209 R. Ahmad, M.S. Hoogeman, S. Quint, et al., "Inter-fraction bladder filling variations and time trends for cervical cancer patients assessed with a portable 3-dimensional ultrasound bladder scanner", 2008 Radiother Oncol (89:172-179)

210 J.S. Chang, H.I. Yoon, H.J. Cha, et al., "Bladder filling variations during concurrent chemotherapy and pelvic radiotherapy in rectal cancer patients: early experience of bladder volume assessment using ultrasound scanner", 2013 Radiat Oncol J (31:41-47)

211 R. Ahmad, M.S. Hoogeman, M. Bondar, et al., "Increasing treatment accuracy for cervical cancer patients using correlations between bladder-filling change and cervix-uterus displacements: proof of principle", 2011 Radiother Oncol (98:340-346)

212 M.L. Bondar, M.S. Hoogeman, J.W. Mens, et al., "Individualized nonadaptive and online-adaptive intensitymodulated radiotherapy treatment strategies for cervical cancer patients based on pretreatment acquired variable bladder filling computed tomography scans", 2012 Int J Radiat Oncol Biol Phys (83:1617-1623)

213 R. Ahmad, L. Bondar, P. Voet, et al., "A margin-of-the-day online adaptive intensity-modulated radiotherapy strategy for cervical cancer provides superior treatment accuracy compared to clinically recommended margins: A dosimetric evaluation", 2013 Acta Oncol (52:1430-1436)

214 S. Van Dyk, K. Narayan, R. Fisher, D. Bernshaw, "Conformal brachytherapy planning for cervical cancer using transabdominal ultrasound", 2009 Int J Radiat Oncol Biol Phys (75:64-70)

215 M. Baker, J.A. Jensen, C.F. Behrens, presented at the Proc. IEEE Ultrasonics Symposium, Prague, 2013 (unpublished).

216 M. Baker, J.A. Jensen, C.F. Behrens, presented at the Proc. SPIE 9040, Medical Imaging 2014: Ultrasonic Imaging and Tomography, San Diego, California, USA, 2014 (unpublished).

217 X. Yang, S. Tridandapani, J.J. Beitler, et al., "Ultrasound GLCM texture analysis of radiation-induced parotidgland injury in head-and-neck cancer radiotherapy: an in vivo study of late toxicity", 2012 Med Phys (39:5732-5739)

218 C. Kelly, L. Rivard, S. Salvi, et al., "Surveillance following head, neck, and chest radiotherapy: thyroid ultrasound monitoring for secondary thyroid malignancy", 2013 Pediatr Blood Cancer (60:140-142)

219 D. Fraser, P. Fava, F. Cury, et al., presented at the Proc. SPIE 6509, Medical Imaging 2007: Visualization and Image-Guided Procedures, , San Diego, CA, 2007 (unpublished).

220 M. Zielinski, A. Szlubowski, M. Kolodziej, et al., "Comparison of endobronchial ultrasound and/or endoesophageal ultrasound with transcervical extended mediastinal lymphadenectomy for staging and restaging of non-small-cell lung cancer", 2013 J Thorac Oncol (8:630-636)

221 T. Terunuma, T. Sakae, Y. Hayakawa, et al., "Waveform simulation based on 3D dose distribution for acoustic wave generated by proton beam irradiation", 2007 Med Phys (34:3642-3648)

222 L. Xiang, B. Han, C. Carpenter, et al., "X-ray acoustic computed tomography with pulsed x-ray beam from a medical linear accelerator", 2013 Med Phys (40:010701) 
SYSTEM ROBUSTNESS 



\section{CHAPTER}

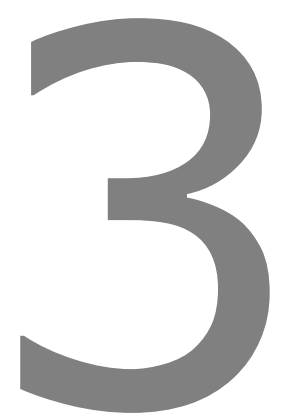

Consequences of intermodality registration errors for intramodality 3D ultrasound IGRT

Skadi van der Meer, Enrica Seravalli, Davide Fontanarosa, Esther J. Bloemen-van Gurp and Frank Verhaegen

Published in: Technology in Cancer Research \& Treatment June 5: 1-7, 2015. DOI: $10.1177 / 1533034615588198$ 


\begin{abstract}
Intramodality ultrasound image-guided radiotherapy systems compare daily ultrasound to reference ultrasound images. Nevertheless, because the actual treatment planning is based on a reference computed tomography image, and not on a reference ultrasound image, their accuracy depends partially on the correct intermodality registration of the reference ultrasound and computed tomography images for treatment planning. The error propagation in daily patient positioning due to potential registration errors at the planning stage was assessed in this work. Five different scenarios were simulated involving shifts or rotations of ultrasound or computed tomography images. The consequences of several workflow procedures were tested with a phantom setup. As long as the reference ultrasound and computed tomography images are made to match, the patient will be in the correct treatment position. In an example with a phantom measurement, the accuracy of the performed manual fusion was found to be $\leq 2 \mathrm{~mm}$. In clinical practice, manual registration of patient images is expected to be more difficult. Uncorrected mismatches will lead to a systematically incorrect final patient position because there will be no indication that there was a misregistration between the computed tomography and reference ultrasound images. In the treatment room, the fusion with the computed tomography image will not be visible and based on the ultrasound images the patient position seems correct.
\end{abstract}




\section{INTRODUCTION}

Current quantitative ultrasound (US) image-guided radiotherapy (IGRT) systems ${ }^{1}$ can be divided into two categories: intermodality and intramodality systems (Figure 1). With the intermodality systems, such as the B-mode acquisition and targeting system(BAT, Best Nomos, Pittsburg, Pennsylvania), ${ }^{2,3}$ the US images acquired at the treatment stage $\left(\right.$ US $\left._{\mathrm{tx}}\right)$ are compared to the reference computed tomography (CT) image acquired during the simulation stage. The intramodality approach entails comparison of an $\mathrm{US}_{\mathrm{tx}}$ image with a reference US (US ref) image, acquired at the time of CT simulation, and therefore compares like with like. An example of this methodology is the Clarity system (Elekta, Stockholm, Sweden). ${ }^{4,5}$

Although intramodal alignment at the treatment stage is less sensitive to errors compared to the inter-imaging modality discrepancies ${ }^{6}$ in intermodality systems, the complete workflow contains extra registration steps and procedures that may increase the error susceptibility. Because the actual treatment planning is based on a reference CT image,

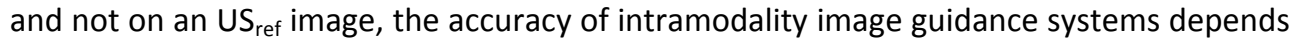
on the correct intermodality registration of the patient's US $_{\text {ref }}$ Scan and the planning CT. In normal procedures, in which the US $\mathrm{S}_{\text {ref }}$ and CT scans are made in direct succession and without any patient motion in between, the image fusion is an automatic process. When this automatic image fusion yields an incorrect match due to, for example, patient motion between the $\mathrm{US}_{\text {ref }}$ and the CT scans, there will be an alignment error between the CT and the US ref Scans, which, if not corrected, could propagate to the stage of the dose delivery.

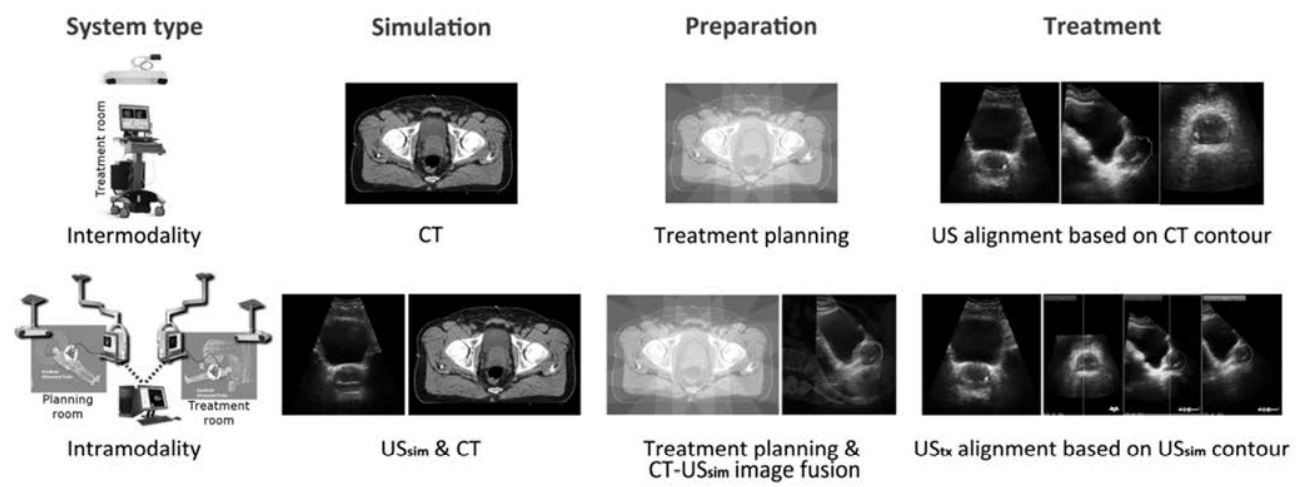

Figure 1: Schematic overview of the procedures during the different stages in the patient workflows for intermodality (top) and intramodality (bottom) ultrasound image-guided radiotherapy (US IGRT) systems. Images reproduced with kind permission of Fontanarosa et al. ${ }^{1}$ System type images courtesy of Best Nomos (top) and Elekta (bottom).

The current manufacturer-recommended procedure is to rescan the CT and the US image in case of a known mismatch. However, since this is very time consuming, especially when it is not noticed immediately, an alternative approach to register the $\mathrm{US}_{\text {ref }}$ and CT images could be valuable. This could consist of manual shifting of either the CT or the US ref image to accomplish a best fit between the two image modalities. This manual shifting relies on 
visual interpretation of common landmarks (e.g., bladder neck) and thus adds an element of user dependence that may affect the accuracy of the positioning of intramodality systems.

In this work, we investigate the potential consequences for the intramodality approach when the $\mathrm{CT}$ and $\mathrm{US}_{\text {ref }}$ Scans are not acquired in the same patient position, simulating uncertainties that may occur in daily practice due to a variety of reasons (e.g., patient motion or laser offset). We will show how errors may propagate to the dose delivery stage, potentially jeopardizing patient safety. ${ }^{7,8}$ In particular, this may also occur in cases where registration errors remain undetected. Methods for assessing potential errors and their causes proactively are very useful approaches for identifying and reducing errors and malfunctions ${ }^{9}$ and should become more standard practice. With simple examples, we try to give a better understanding of the system and prevent errors due to simple mistakes or misinterpretations.

\section{MATERIALS AND METHODS}

\section{Intramodality 3-dimensional US IGRT System}

The Clarity 3-dimensional (3D) US system consists of two US units, one in the CT room (USSim) and another one in the treatment room (US-Guide). At both units, a ceiling-mounted optical infrared position tracking system continuously tracks the US probe with reflective markers. This tracking system is calibrated through a room and probe calibration procedure with a dedicated calibration phantom, accomplishing the 3D reconstruction of the US volume referenced to the same room coordinate system with a tolerance level of $1 \mathrm{~mm}$ for each room as stated by the manufacturer.

During simulation, the patient is positioned on the CT table, and three external fiducials (metal markers) are patched on the patient's skin at the outer intersection points of the room lasers (defining the CT reference point). The CT and $\mathrm{US}_{\text {ref }}$ images are acquired in quick succession with the patient in the same position. (The US scan is acquired with the patient aligned to the positioning laser outside the CT-bore. Directly following this US scan, the CT table is shifted inside the bore and a CT is acquired. The lasers correspond to the $\mathrm{CT}$ reference point through a calibration procedure.) On a workstation the simulation isocenter is identified on the CT, and the registered $\mathrm{US}_{\text {ref }}$ is overlayed to form a CT-US fusion. Once the treatment plan is complete, the isocenter information is transferred to the treatment unit and transformed from simulation to treatment coordinates. An initial isoshift (from the US ref reference point to the radiation beams isocenter location) is reported and is based on the intermodality fusion of the patient US $\mathrm{S}_{\text {ref }}$ and CT images.

For every radiotherapy fraction, the patient is aligned to the room lasers, and the acquired $U_{\text {tx }}$ scan is matched to the reference guidance structure of US ${ }_{\text {ref }}$ scan. Based on this match, the patient is shifted in three directions to realign the target with the radiation beams. $^{10}$ 


\section{Workflow}

We simulated five different scenarios compared to correct alignment (Table 1), mimicking errors that could occur during the simulation stage caused by, for example, laser offsets, patient motion (translations and rotations), or inaccurate room calibrations.

Table 1. The five Different Scenarios in Comparison to the Correct Alignment on the Room Lasers.

\begin{tabular}{|c|c|c|c|}
\hline Scenario & $\mathrm{CT}$ & & US $_{\text {ref }}$ \\
\hline Reference situation & Correct alignment & & Correct alignment \\
\hline 1 & Shifted & & Correct alignment \\
\hline 2 & Correct alignment & & Shifted \\
\hline 3 & Shifted & $=$ & Shifted \\
\hline 4 & Shifted & $\neq$ & Shifted \\
\hline 5 & Correct alignment & & Rotated \\
\hline
\end{tabular}

The CT and $\mathrm{US}_{\text {ref }}$ images are assumed to be fused on the Clarity workstation using the alignment of the reference point (Figure 2). The $U_{\text {ref }}$ reference point is defined as the intersection point of the room lasers (point $L$ at intersection of dashed lines). The CT reference point is defined as either the intersection of the horizontal line connecting the lateral metal markers on the phantom/patient and the vertical line through the anterior marker (point $\mathrm{MM}$ ) or the coordinates of the intersection of the room lasers (point $\mathrm{L}$ ). The $\mathrm{MM}$ is same as the reference point used by the treatment planning system to propose an initial isoshift (from the reference point $\mathrm{MM}$ to the treatment isocenter).

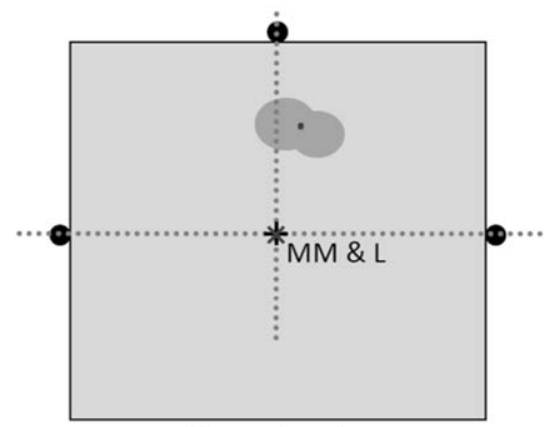

Aligned on Lasers

Metal marker

$(+, M M)$ Metal marker $\mathrm{CT}$ reference point

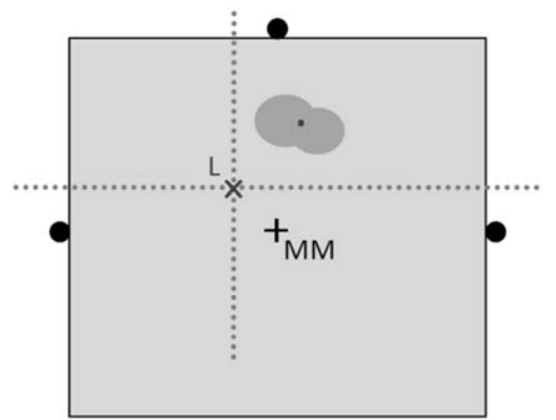

Not aligned on Lasers

$(\ldots . . .$.$) Laser line$

$(\mathrm{X}, \mathrm{L})$ Laser reference point

Target volume with treatment isocenter

Figure 2: Aligned and nonaligned patient/phantom positions. The origin of the ultrasound (US) system is defined with a room calibration and is made to coincide with the room laser intersection point (L). The origin of the CT can be defined as $L$ or as the metal marker intersection point (MM) of the three markers. L and MM are identical when the patient is correctly aligned. A shift between these two occurs when there is a laser offset, when the patient moved in between scans, or when there is an incorrect room calibration of the US system. 


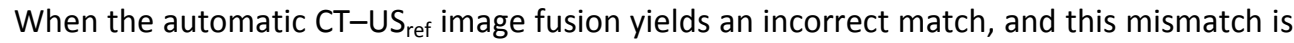
not corrected before the start of the treatment, there will be a systematic misalignment

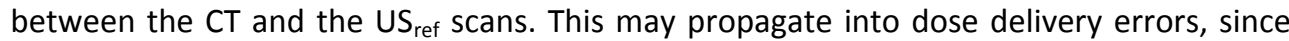
this is based on the planning CT and the patient positioning is based on US guidance. When mismatches between the $\mathrm{CT}$ and $\mathrm{US}_{\text {ref }}$ scans are detected, the recommended (Clarity system user guide), yet time-consuming, procedure is to reacquire the scans. An alternative match correction approach is manual shifting of either the CT or the US ref image. We also assessed the consequences of not applying a correction in case of a mismatch that could occur, for example, in case the mismatch was not noticed or deliberately left uncorrected.

\section{RESULTS}

The consequences of the different actions in the first scenario are shown in Figure 3. The $\mathrm{US}_{\text {ref }}$ is made with the phantom/patient correctly aligned on the room lasers, while the CT was made with a misalignment. There are two choices for the CT reference point. If one chooses $\mathrm{MM}$ as the $\mathrm{CT}$ reference point, the automatic fusion will be correct and the proposed shifts from both the Clarity workstation and the US-Guide will direct the patient to the correct treatment position. If $L$ is chosen as the CT reference point, the automatic fusion will not result in a correct alignment between the CT and the US $\mathrm{S}_{\text {ref. }}$ Three actions can then be taken. The first one is obtaining a correct match by manual shifting of the CT toward the US $\mathrm{Sef}_{\text {re }}$ This will result in correct treatment alignment for both the initial and the US-Guide proposed shifts. The second choice of action is shifting the $\mathrm{US}_{\text {ref }}$ toward the CT instead of the other way around. This will result in an incorrect initial proposed isoshift. Fortunately, since the $\mathrm{US}_{\text {ref }}$ and CT images are made to match, the position of the isocenter and radiation beams is correct. When the reference guidance structure will be made to match $U_{\mathrm{tx}}$, US-Guide will still direct the patient to the correct treatment position. The final option is not to correct the CT-US ref misalignment. However, this will result in incorrect importing of the isocenter and radiation beams. Both are imported relative to the CT coordinates, and since the target on the $\mathrm{US}_{\text {ref }}$ is not made to match with the target on the $\mathrm{CT}$, the isocenter of the $\mathrm{US}_{\text {ref }}$ will not be in the correct position in the US target volume. As a consequence, both the initial and the US-Guide proposed shifts will not result in a correct patient treatment position. There will be no indication that there was a misregis-

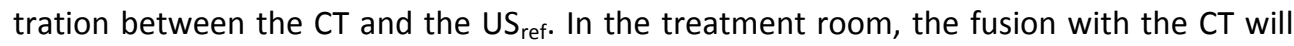
not be visible and based on the US images, the patient position seems correct. The errors will be made systematically, which could result in dose delivery errors.

The evaluation of the action consequences of all the scenarios is shown in Table 2. For the "Consequences for positioning" as can be seen in the right column of the table, it is assumed that the patient is positioned on the room lasers of the treatment room. However, the correct treatment alignment is independent of the initial patient position in the treatment room. The starting position in the treatment room will have an influence on the proposed US-Guide-based correction shift, yet the final treatment position will be the same. In case of rotational differences, none of the matching actions will lead to correct 
position guidance. In that case, the only solution is rescanning (both CT and US $\mathrm{Sef}_{\text {) }}$ the patient.

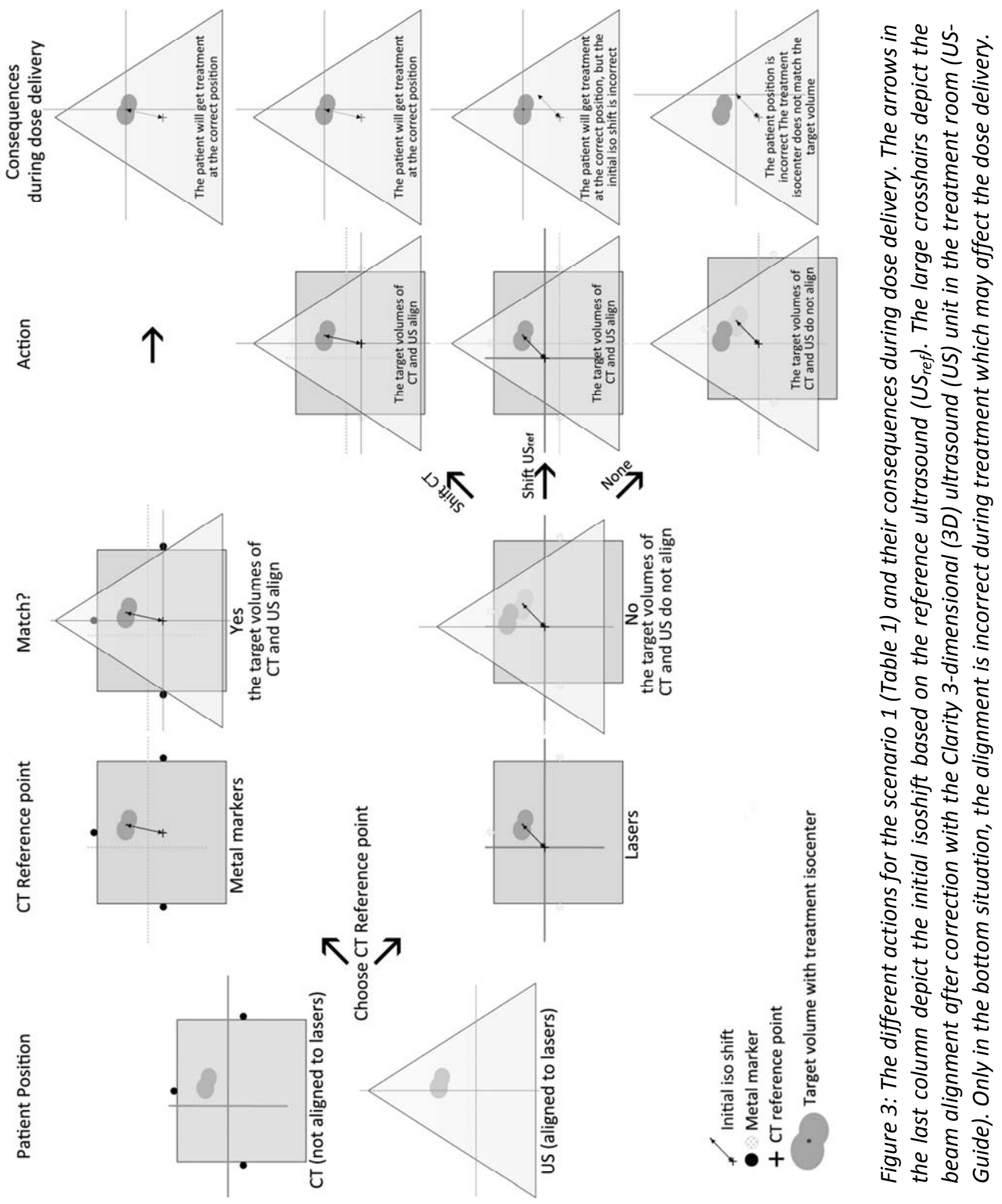

For translational shifts between the CT and the US ref, as long as the patient $\mathrm{US}_{\text {ref }}$ and CT images are made to match, the isocenter from the CT is copied to the correct location on the US ref, and US-Guide will direct the patient to the correct treatment position. Uncor- 
rected mismatches will lead to a final incorrect patient position. Because the actual treatment planning is based on a reference $\mathrm{CT}$, and not on an $\mathrm{US}_{\text {ref, }}$ it is preferred to use the CT registered with the metal markers and shift the $\mathrm{US}_{\text {ref }}$ to match the CT when manual fusion is employed for the match. With this approach, the initial isoshift calculated with the $U_{\text {ref }}$ scan will be correct and will not question the US-Guide-based shift.

\section{DISCUSSION}

In normal procedures, the intermodality $\mathrm{CT}-\mathrm{US}_{\text {ref }}$ image fusion is an automated process, accomplished through a phantom calibration procedure. As long as the US ref $_{\text {and }}$ CT scan are made in direct succession, without any patient motion in between, and all the calibrations and laser alignments are correct, even speed of sound (SOS) aberrations ${ }^{11-14}$ will be

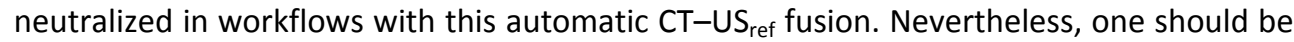

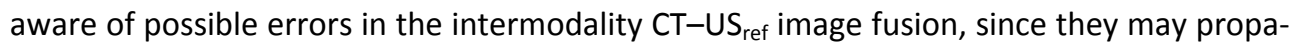
gate to every treatment session as systematic dose delivery error.

In the case of SOS aberrations, correct automatic intermodality fusion could give a visual mismatch between the $\mathrm{CT}$ and the $\mathrm{US}_{\text {ref }}$ images. Yet, this mismatch is not because of patient motion, laser offsets, or incorrect calibration but due to SOS errors resulting in apparent shifts in the US $\mathrm{S}_{\text {ref }}$ image. If the mismatch in the CT-US $\mathrm{S}_{\text {ref }}$ fusion was corrected in this case, an error of another nature would be introduced instead of correcting for the mismatch error due to a shift. Therefore, the consequences described in Table 2 are only applicable to SOS corrected US images. ${ }^{12,15}$ The user should be aware that during treatment positioning when the following intramodality registration takes part, both US images should be SOS corrected or not. If SOS-corrected US images are made to match with nonSOS-corrected images, errors are introduced. For the example with a phantom measurement in Table A (Supplementary Material), a SOS correction was not necessary, since the calibration phantom is made of materials that have the same SOS as is assumed by the US

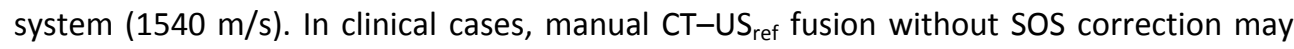
result in residual positioning errors or may even introduce them. This is a complex issue that was recently studied by Fontanarosa et al. ${ }^{13,14}$

Furthermore, the accuracy of manual fusion depends on the matching skills of the operator and will therefore affect the accuracy of the positioning. For the intramodality matching, the inter-operator variability was found to be $1.3 \mathrm{~mm}, 1.4 \mathrm{~mm}$, and $1.8 \mathrm{~mm}$, respectively, in the left-right, anterior-posterior, and superior-inferior direction. ${ }^{10}$ For manual intermodality matching, this variability is not expected to be smaller since the tissue boundaries in CT and US are depicted very differently. ${ }^{16-19}$ Although some of these errors might be intercepted by the safety margins used for treatment planning, and therefore will not always propagate to actual dose delivery errors, the recommended procedure in case of patient motion is always to rescan the patient with both CT and US ref. Registration errors may still go undetected in which case Table 2 describes how errors will propagate; a necessity for safety assessments purposes. Table 2 may also serve as a guide to make US systems more robust against error propagation. Better understanding of the system can prevent misinterpretations and avoid mistakes. 
Table 2: Actions and Consequences of Registration Errors in the CT-US ref Fusion. ${ }^{a}$

\begin{tabular}{|c|c|c|c|c|}
\hline \multicolumn{5}{|c|}{ Translations } \\
\hline Patient Position & CT Ref. point & Match & Action to get a match & Consequence for positioning \\
\hline \multirow{4}{*}{$\begin{array}{l}\text { CT Shifted, } \\
\text { US }_{\text {ref }} \text { on laser }\end{array}$} & $\mathrm{MM}$ & Yes & None & $\begin{array}{l}\text { Initial iso and US-Guide based shifts are both } \\
\text { correct }\end{array}$ \\
\hline & \multirow{3}{*}{ L } & \multirow{3}{*}{ No } & CT shifted to US $\mathrm{Sef}_{\text {ref }}$ & $\begin{array}{c}\text { Initial iso and US-Guide based shifts are } \\
\text { both correct }\end{array}$ \\
\hline & & & US $_{\text {ref }}$ shifted to CT & $\begin{array}{l}\text { Initial iso shift is incorrect, while the } \\
\text { US-Guide based shift is correct }\end{array}$ \\
\hline & & & None & $\begin{array}{l}\text { Initial iso and US-Guide based shifts are both } \\
\text { incorrect }\end{array}$ \\
\hline \multirow{3}{*}{$\begin{array}{l}\text { CT on laser, } \\
\text { US }_{\text {ref }} \text { Shifted }\end{array}$} & \multirow{3}{*}{$\begin{array}{l}\text { MM \& L give } \\
\text { same result }\end{array}$} & \multirow{3}{*}{ No } & CT shifted to US $\mathrm{Sef}_{\mathrm{r}}$ & $\begin{array}{l}\text { Initial iso shift is incorrect, while the } \\
\text { US-Guide based shift is correct }\end{array}$ \\
\hline & & & $\mathrm{US}_{\text {ref }}$ shifted to CT & $\begin{array}{l}\text { Initial iso and US-Guide based shifts are both } \\
\text { correct }\end{array}$ \\
\hline & & & None & $\begin{array}{l}\text { Initial iso shift is correct, while the } \\
\text { US-Guide based shift is incorrect }\end{array}$ \\
\hline \multirow{4}{*}{$\begin{array}{l}\mathrm{CT} \text { and } \mathrm{US}_{\text {ref }} \\
\text { Shifted }\end{array}$} & \multirow{3}{*}{ MM } & \multirow{3}{*}{ No } & CT shifted to US $\mathrm{S}_{\text {ref }}$ & $\begin{array}{l}\text { Initial iso shift is incorrect, while the } \\
\text { US-Guide based shift is correct }\end{array}$ \\
\hline & & & $\mathrm{US}_{\text {ref }}$ shifted to CT & $\begin{array}{l}\text { Initial iso and US-Guide based shifts are both } \\
\text { correct }\end{array}$ \\
\hline & & & None & $\begin{array}{l}\text { Initial iso shift is correct, while the } \\
\text { US-Guide based shift is incorrect }\end{array}$ \\
\hline & L & Yes & None & $\begin{array}{l}\text { Initial iso shift is incorrect, while the } \\
\text { US-Guide based shift is correct }\end{array}$ \\
\hline \multirow{6}{*}{$\begin{array}{l}\text { CT and US } \\
\text { Shifted with } \\
\text { different shifts }\end{array}$} & \multirow{3}{*}{ MM } & \multirow{3}{*}{ No } & CT shifted to $U_{\text {ref }}$ & $\begin{array}{l}\text { Initial iso shift is incorrect, while the } \\
\text { US-Guide based shift is correct }\end{array}$ \\
\hline & & & US $_{\text {ref }}$ shifted to CT & $\begin{array}{l}\text { Initial iso and US-Guide based shifts are both } \\
\text { correct }\end{array}$ \\
\hline & & & None & $\begin{array}{l}\text { Initial iso shift is correct, while the } \\
\text { US-Guide based shift is incorrect }\end{array}$ \\
\hline & \multirow{3}{*}{$\mathrm{L}$} & \multirow{3}{*}{ No } & CT shifted to US ref & $\begin{array}{l}\text { Initial iso shift is incorrect, while the } \\
\text { US-Guide based shift is correct }\end{array}$ \\
\hline & & & US $_{\text {ref }}$ shifted to CT & $\begin{array}{l}\text { Initial iso shift is incorrect, while the } \\
\text { US-Guide based shift is correct }\end{array}$ \\
\hline & & & None & $\begin{array}{l}\text { Initial iso and US-Guide based shifts are both } \\
\text { incorrect }\end{array}$ \\
\hline \multicolumn{5}{|c|}{ Rotations } \\
\hline \multirow{2}{*}{$\begin{array}{l}\text { Any rotational } \\
\text { difference } \\
\text { between } \mathrm{CT} \text { and } \\
\text { US }_{\text {ref }}\end{array}$} & \multirow{2}{*}{$\begin{array}{l}\text { MM \& L give } \\
\text { same result }\end{array}$} & \multirow[t]{2}{*}{ No } & $\begin{array}{l}\mathrm{CT} \text { rotated to } \mathrm{US}_{\text {ref, }} \\
\mathrm{US}_{\text {ref }} \text { rotated to } \mathrm{CT}\end{array}$ & $\begin{array}{l}\text { At this moment the system can not handle } \\
\text { rotations in the fusion. } \\
\text { No positioning guidance }\end{array}$ \\
\hline & & & None & $\begin{array}{l}\text { Initial iso and US-Guide based shifts are both } \\
\text { incorrect }\end{array}$ \\
\hline
\end{tabular}

Abbreviations: $M M$, intersection of the lines connecting the metal markers; $L$, intersection point of the room lasers; CT, computed tomography; USref, reference US; US-Guide: Clarity 3DUS unit in the treatment room.

${ }^{a}$ In light gray, only the initial isoshift is incorrect. In the dark gray highlighted situations the final patient position, that is, the treatment position, is incorrect, which leads to erroneous dose delivery. 


\section{CONCLUSION}

The Clarity 3DUS guidance system is a robust IGRT device that guides the patient to the correct treatment position under the following conditions: the metal markers are used for the registration of the CT; SOS aberrations are taken into account ${ }^{12,15}$ in intermodality image fusion, and preferably also during the intramodality procedures (both US images); and the CT scan and (SOS corrected) US $_{\text {ref }}$ scan match. In the case of a mismatch, it is advised to rescan the patient with both $\mathrm{CT}$ and $\mathrm{US}_{\text {ref }}$ in direct succession with good patient stabilization to prevent patient motion in between the scans.

If one opts not to rescan, one could try to obtain a correct CT and US ref fusion by shift-

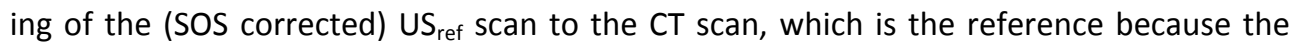

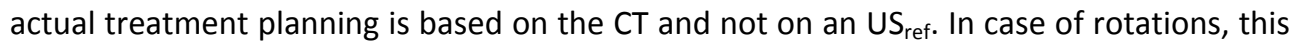
will not result in a correct match since the Clarity system cannot correct for rotations. Nonetheless, the accuracy of manual fusion depends on the matching skills of the operator. With an example with a phantom measurement (Supplementary Material), the accuracy of the manual fusion performed by an experienced user was found to be $\leq 2 \mathrm{~mm}$, but in the case of a patient, the manual fusion may be more difficult. Therefore, in the case of a mismatch or rotations, we always recommend rescanning of the patient (both CT and $\mathrm{US}_{\text {ref }}$ ). One should be aware that registration errors may go undetected.

\section{ACKNOWLEDGMENTS}

The authors like to thank Elekta for their support and in particular $\operatorname{Dr}$ M. Lachaine for discussion

\section{REFERENCES}

1 D. Fontanarosa, S. van der Meer, J. Bamber, et al., "Review of ultrasound image guidance in external beam radiotherapy: I. Treatment planning and inter-fraction motion management", 2015 Physics in Medicine and Biology (60:R77)

2 J. Lattanzi, S. McNeeley, W. Pinover, et al., "A comparison of daily CT localization to a daily ultrasoundbased system in prostate cancer", 1999 International journal of radiation oncology, biology, physics (43:719-725)

3 J. Boda-Heggemann, P. Mennemeyer, H. Wertz, et al., "Accuracy of ultrasound-based image guidance for daily positioning of the upper abdomen: an online comparison with cone beam CT", 2009 International journal of radiation oncology, biology, physics (74:892-897)

4 F.L. Cury, G. Shenouda, L. Souhami, et al., "Ultrasound-based image guided radiotherapy for prostate cancer: comparison of cross-modality and intramodality methods for daily localization during external beam radiotherapy", 2006 International journal of radiation oncology, biology, physics (66:1562-1567)

5 E. Mayyas, I.J. Chetty, M. Chetvertkov, et al., "Evaluation of multiple image-based modalities for imageguided radiation therapy (IGRT) of prostate carcinoma: A prospective study", 2013 Medical physics (40:041707)

6 J.A. Molloy, G. Chan, A. Markovic, et al., "Quality assurance of U.S.-guided external beam radiotherapy for prostate cancer: report of AAPM Task Group 154", 2011 Medical physics (38:857-871)

7 W.R. Hendee, M.G. Herman, "Improving patient safety in radiation oncology", 2011 Practical Radiation Oncology (1:16-21)

8 D.A. Jaffray, K.M. Langen, G. Mageras, et al., "Safety considerations for IGRT: Executive summary", 2013 Pract Radiat Oncol (3:167-170) 
9 E.C. Ford, R. Gaudette, L. Myers, et al., "Evaluation of safety in a radiation oncology setting using failure mode and effects analysis", 2009 International journal of radiation oncology, biology, physics (74:852-858)

10 S. van der Meer, E. Bloemen-van Gurp, J. Hermans, et al., "Critical assessment of intramodality 3D ultrasound imaging for prostate IGRT compared to fiducial markers", 2013 Medical physics (40:071707)

11 B.J. Salter, B. Wang, M.W. Szegedi, et al., "Evaluation of alignment error due to a speed artifact in stereotactic ultrasound image guidance", 2008 Phys Med Biol (53:N437-445)

12 D. Fontanarosa, S. van der Meer, E. Harris, F. Verhaegen, "A CT based correction method for speed of sound aberration for ultrasound based image guided radiotherapy", 2011 Medical physics (38:2665-2673)

13 D. Fontanarosa, S. van der Meer, E. Bloemen-van Gurp, et al., "Magnitude of speed of sound aberration corrections for ultrasound image guided radiotherapy for prostate and other anatomical sites", 2012 Medical physics (39:5286-5292)

14 D. Fontanarosa, S. van der Meer, F. Verhaegen, "On the significance of density-induced speed of sound variations on US-guided radiotherapy", 2012 Medical physics (39:6316-6323)

15 D. Fontanarosa, S. Pesente, F. Pascoli, et al., "A speed of sound aberration correction algorithm for curvilinear ultrasound transducers in ultrasound-based image-guided radiotherapy", 2013 Phys Med Biol (58:13411360)

16 V. Narayana, P.L. Roberson, A.T. Pu, et al., "Impact of differences in ultrasound and computed tomography volumes on treatment planning of permanent prostate implants", 1997 International journal of radiation oncology, biology, physics (37:1181-1185)

17 S.C. Hoffelt, L.M. Marshall, M. Garzotto, et al., "A comparison of CT scan to transrectal ultrasoundmeasured prostate volume in untreated prostate cancer", 2003 International journal of radiation oncology, biology, physics (57:29-32)

18 J.A. Molloy, S. Srivastava, B.F. Schneider, "A method to compare supra-pubic ultrasound and CT images of the prostate: technique and early clinical results", 2004 Medical physics (31:433-442)

19 D.A. Kuban, L. Dong, R. Cheung, et al., "Ultrasound-based localization", 2005 Seminars in radiation oncology $(15: 180-191)$ 
$66 \mid$ Chapter 3

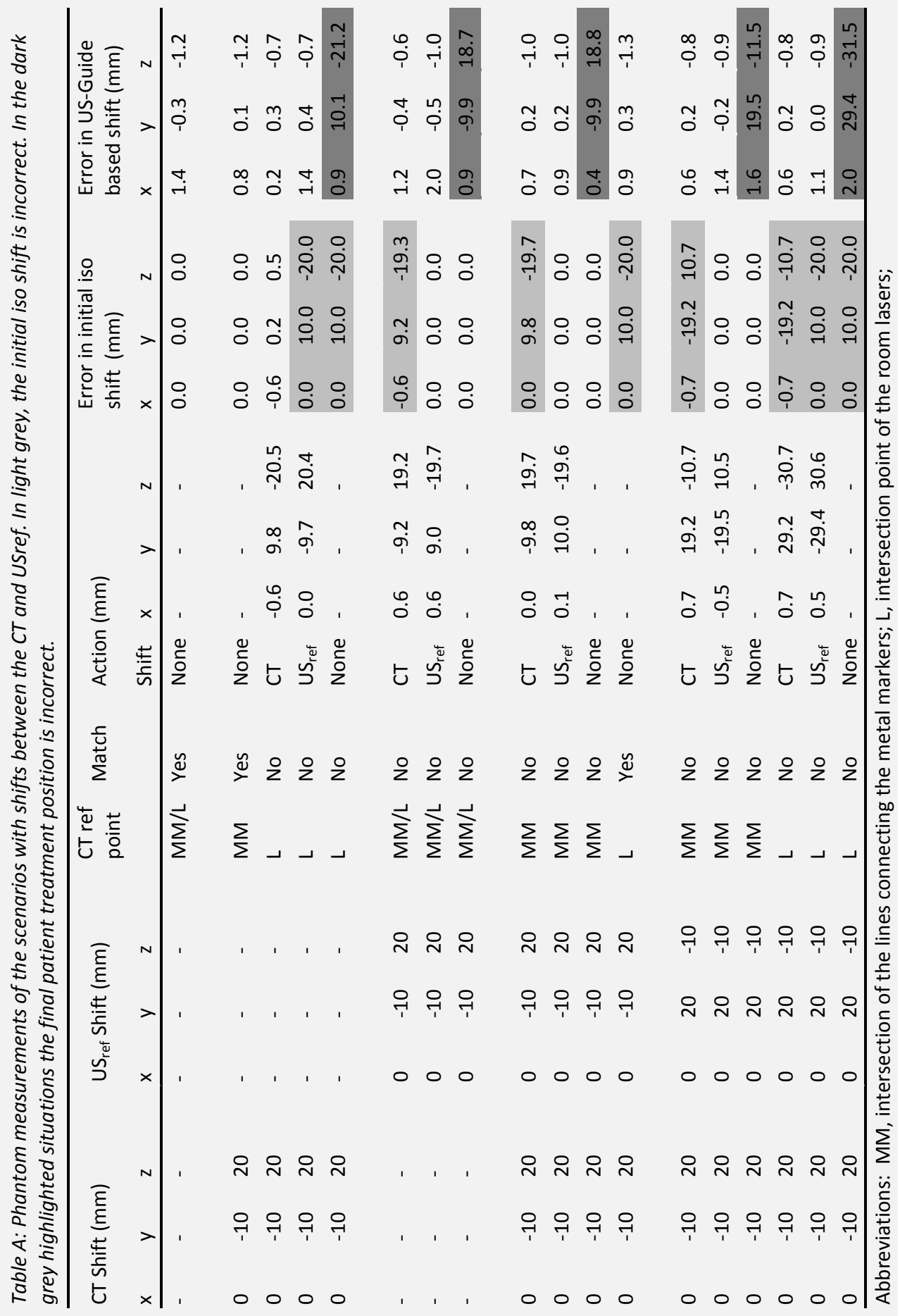




\section{SUPPLEMENTAL MATERIAL}

\section{Materials and methods}

We performed USref and CT scans of the calibration phantom, containing multiple rods and spheres, in four positions:

1) aligned with the $\mathrm{CT}$ room lasers

2) not aligned with the CT room lasers with a translation of $10 \mathrm{~mm}$ inferior and 20 $\mathrm{mm}$ posterior

3) not aligned with the CT room lasers with a translation of $20 \mathrm{~mm}$ superior and 10 $\mathrm{mm}$ anterior

In the third position, only an US scan was performed. Cases 2-3 mimic errors during the simulation stage caused by for example laser offsets, patient motion (translations and rotations) or inaccurate room calibrations.

With these scans we simulated the first four scenarios compared to correct alignment, like was done in Table 1.

To assess the alternative approaches, several positioning references with different fusions were tested at the US-Guide. The markers of the phantom were aligned on the treatment room lasers, and the UStx scan was acquired. The reference USref contour was made to match UStx and a correction shift was calculated. If this US-Guide correction shift was equal to the proposed shift from the treatment planning system, it was assumed to be correct. A $2 \mathrm{~mm}$ tolerance level on the difference between the two shifts was used to account for the accuracy of the system and the manual fusion.

\section{Results}

The results from the phantom measurements are shown in Table A. The last three columns contain the difference between the US-Guide based shift and the theoretical correct shift from the treatment planning system. Notice that, when the US-Guide positioning is correct, the error may not always equal zero. This is due to the limited accuracy of the manual matching, the phantom repositioning and the system accuracy.

For most situations in which the CT and USref match, with either automatic or corrected manual fusion, the accuracy of the US-Guide was $\leq 1.4 \mathrm{~mm}$, conform to the manufacturer's stated overall system accuracy tolerance. 

CLINICAL STUDIES 



\section{CHAPTER}

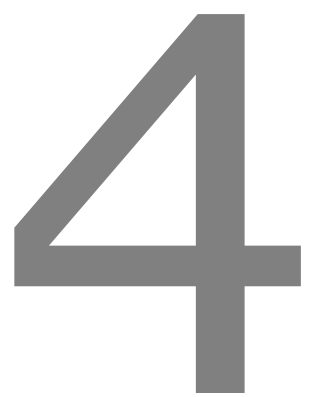

Critical assessment of intramodality 3D ultrasound imaging for prostate IGRT compared to fiducial markers

Skadi van der Meer, Esther Bloemen-van Gurp, Jolanda Hermans, Robert Voncken, Denys Heuvelmans, Carol Gubbels, Davide Fontanarosa, Peter Visser, Ludy Lutgens, Francis van Gils and Frank Verhaegen

Published in: Medical Physics 40(7):071707-11, 2013.

DOI: $10.1118 / 1.4808359$. 
72 |Chapter 4

\section{ABSTRACT}

Purpose: A quantitative 3D intramodality ultrasound (US) imaging system was verified for daily in-room prostate localization, and compared to prostate localization based on implanted fiducial markers (FMs).

Methods: Thirteen prostate patients underwent multiple US scans during treatment. A total of 376 US scans and 817 matches were used to determine the intra- and interoperator variability. Additionally, eight other patients underwent daily prostate localization using both US and electronic portal imaging (EPI) with FMs resulting in 244 combined US-EPI scans. Scanning was performed with minimal probe pressure and a correction for the speed of sound aberration was performed. Uncertainties of both US and FM methods were assessed. User variability of the US method was assessed.

Results: The overall US user variability is $2.6 \mathrm{~mm}$. The mean differences between US and FM are: $2.5 \pm 4.0 \mathrm{~mm}$ (LR), $0.6 \pm 4.9 \mathrm{~mm}(\mathrm{SI})$, and $-2.3 \pm 3.6 \mathrm{~mm}$ (AP). The intramodality character of this US system mitigates potential errors due to transducer pressure and speed of sound aberrations.

Conclusions: The overall accuracy of US $(3.0 \mathrm{~mm})$ is comparable to our FM workflow (2.2 $\mathrm{mm}$ ). Since neither US nor FM can be considered a gold standard no conclusions can be drawn on the superiority of either method. Because US imaging captures the prostate itself instead of surrogates no invasive procedure is required. It requires more effort to standardize US imaging than FM detection. Since US imaging does not involve a radiation burden, US prostate imaging offers an alternative for FM EPI positioning. 


\section{INTRODUCTION}

To allow for safe delivery of intensity-modulated high radiation doses, image guided radiotherapy (IGRT) has become a crucial paradigm. For prostate treatment, one of the most established methods is to use megavolt (MV) electronic portal imaging (EPI) in combination with implanted fiducial markers (FMs) as a prostate location surrogate. ${ }^{1}$ However, FM implantation is not without risk and not always feasible. Infections and bleeding ${ }^{2,3}$ are well-known complications in this procedure. ${ }^{4,5}$ In addition, the presence of the FMs can cause localized differences between the calculated and delivered dose for various treatment modalities. ${ }^{6-9}$ Various other imaging methods have been investigated to localize the prostate during treatment: prostate implanted electromagnetic transponders, ${ }^{10}$ kilovolt (kV) projection imaging or $\mathrm{MV}-\mathrm{EPI}^{11}$ of the bony anatomy, calcification and soft tissue imaging with cone beam computed tomography $(\mathrm{CBCT}),{ }^{12,13}$ magnetic resonance imaging (MRI), ${ }^{14}$ and ultrasound (US) imaging. ${ }^{15-20}$ In particular, the last three techniques in which the prostate itself is visualized may offer it might be an alternative for prostate localization when FMs are not desirable. The latter technique is currently mostly used as a diagnostic technique for qualitative imaging. An imaging technique employed in IGRT needs to be quantitative, i.e., it must be able to measure positions and volumes of anatomical structures linked to a coordinate system.

The clinically available quantitative US systems for IGRT come in different flavors. The first generation 2DUS systems ${ }^{15,17}$ were intermodality systems, i.e., US images acquired in the treatment room had to be compared to a reference computed tomography (CT) image in several planes. Several publications using 2DUS intermodality prostate localization methods have raised concerns regarding the accuracy of US-based techniques due to issues such as transducer pressure induced prostate motion, ${ }^{21-23}$ speed of sound (SOS) errors, $^{24-27}$ and image quality. Current 3DUS imaging systems frequently still use the intermodality approach. A quantitative intramodality 3DUS system was introduced (Clarity system, Elekta, Stockholm, Sweden) several years ago. The intramodality approach entails that US images acquired in the treatment room are compared to a reference US image acquired at the time of CT simulation. This approach should facilitate matching procedures.

Significant differences between the inter- and intramodality approaches were found in a comparison study. ${ }^{28}$ Task Group 154 of the American Association of Physicists in Medicine provided guidelines ${ }^{29}$ on the quality assurance procedures for using 3DUS devices in daily practice. They recommend an intramodality approach and proper training to address the operator variability and localization accuracy, both during image acquisition and matching procedures to reference images. To date, no reports on operator variability for intramodality 3DUS imaging are available.

In the present study we determined the influence of various factors involved in the workflow of US scanning for prostate cancer patients. We studied the inter- and intraoperator variability of 3DUS intramodality matching, and the influence of inter-operator probe pressure on the prostate position while using a strict bladder filling protocol. The 3DUS prostate localization was compared to MV EPI localization based on FMs in a small patient study. With this study we highlight a number of issues that influence the accuracy 
of both procedures, including an estimation of the magnitude of the SOS aberration for the US procedures, which was documented by us in a number of recent papers. ${ }^{25-27} \mathrm{~A}$ large patient study on US versus FM was not within the scope of this work.

\section{MATERIALS AND METHODS}

\section{D ultrasound IGRT system}

The Clarity 3DUS system is currently the only intramodality US IGRT device available for radiotherapy, i.e., it compares US to US images. To this end the system consists of two US units, one in the CT room (US-Sim) and another one in the treatment room (US-Guide). At both units a ceiling-mounted optical position-tracking system continuously tracks the US probe with reflective markers. This tracking system is calibrated through a room and probe calibration procedure with a dedicated calibration phantom, accomplishing the 3D reconstruction of the scanned US volume referenced to the same room coordinate system as the CT images.

According to the specifications of the vendor, the system has an uncertainty of $1 \mathrm{~mm}$ for the US-Sim and $1 \mathrm{~mm}$ for US-Guide. Assuming these can be added quadratically, this would lead to a combined uncertainty for the whole quality assurance procedure (i.e., calibration, phantom positioning, image tracking, image fusion, etc.) of $1.4 \mathrm{~mm}$. Monthly quality control (QC) is warranted to ensure that the system uncertainty does not exceed this level (From March 2010 until March 2011, our average QC results for the whole system were $0.74 \pm 0.57 \mathrm{~mm}$ ).

For the Clarity workflow, all patients received a reference US scan (US $S_{\text {sim }} ; 3.5 \mathrm{MHz}$ ) immediately before or after the planning CT-scan (SOMATOM Sensation Open, Syngo CT 2006A, Siemens, Germany; voxels: $1 \times 1 \mathrm{~mm}^{2} \times 3 \mathrm{~mm}$ slice thickness) at the simulation stage. Both scans were performed in the exact same supine patient position, stabilized with knee fix and foot support, resulting in a correct automatic fusion of the US sim $_{\text {scan to }}$ the planning CT. At the US workstation, a guidance structure is delineated semiautomatically on the $\mathrm{US}_{\mathrm{Sim}}$ scan, to define a positioning reference (reference positioning volume, RPV). In the case of prostate cancer patients, the RPV is most commonly the prostate itself. If the pubic bone casts a large shadow over the prostate, the part of the prostate that is still easily distinguishable on US $_{\text {sim }}$ is used as the RPV. The treatment isocenter and the radiation beams are imported. At treatment stage, the RPV volume is copied onto the current daily US scan ( $\mathrm{US}_{\mathrm{tx}} ; 3.5 \mathrm{MHz}$ ) and moved manually in three orthogonal directions by the operator until it corresponds to the current prostate position (Figure 1). An automatic adapt function adjusts the reference contour elastically to the gray level boundaries of the prostate in the $U_{\mathrm{tx}} \operatorname{scan}^{30,31}$ To obtain the optimal overlap of both volumes, the calculated shift is based on surface matching where segments that fall on clearly visible edges have a high weight while elements on less clearly visible edges have a low weight. In this work, none of the applied patient correction shifts were based on the US images. The US images were collected for comparison purposes only and therefore retrospectively matched and analyzed to minimize the time burden on the patients. 
The normal workflow would entail US-based patient shifts in three directions to realign the target with the radiation beams. Before shifting, a couch position indicator with reflectors is then mounted on the treatment table allowing the ceiling-mounted camera to monitor and validate the correction with the tracking system. No corrective rotations are currently performed.
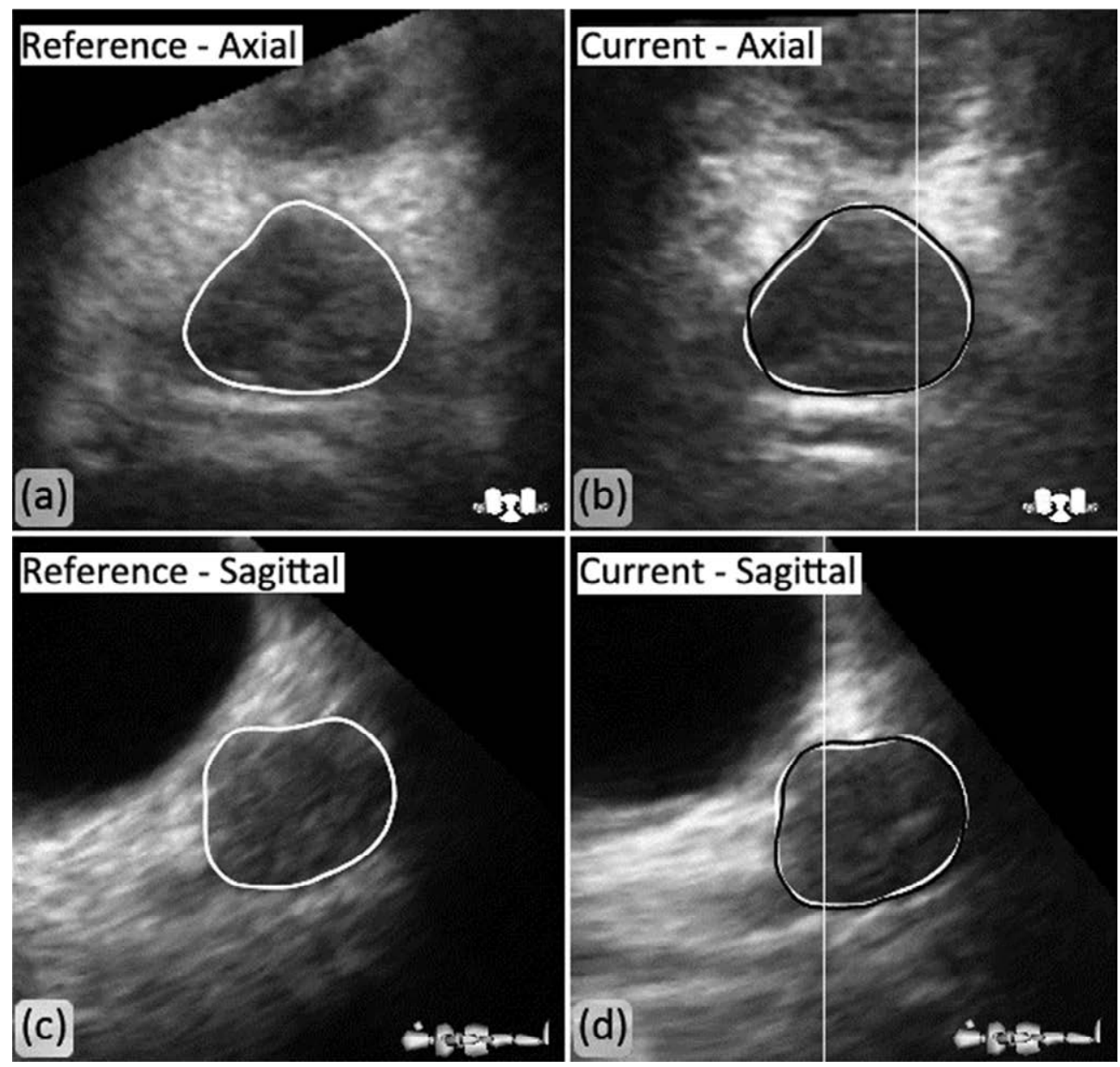

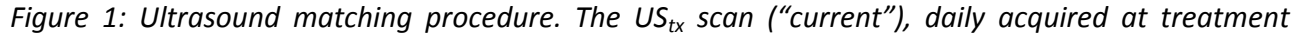
stage [axial view (b) and sagittal view (d)], is matched to the reference $U S_{\text {sim }}$ scan [axial view (a) and sagittal view (c)], which was made during simulation stage. The RPV [white contours in (a) and (c)] on the reference scan is the prostate delineated during the preparation process on the workstation. At treatment stage, this contour is copied to $U S_{t x}$ and moved by the operator to correspond to the current position of the prostate [white contours in (b) and (d)]. An automatic adapt function adjusts the reference contour to the gray level boundaries of the prostate in the current scan [black contours in (b) and (d)] to obtain the best volume fit from which the shift is calculated. 


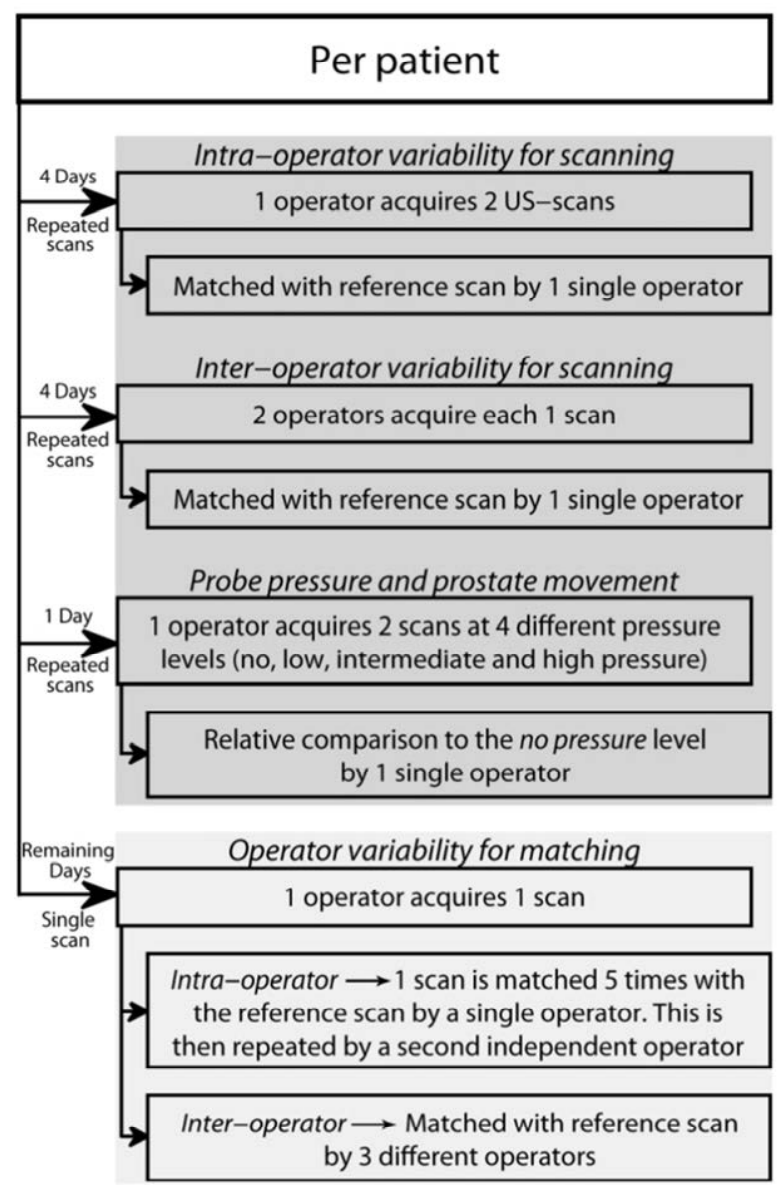

Figure 2: Overview of the scanning and matching variability tests. On the days with repeated scans (dark gray), the focus is on the scan variability. On the days with single scans (light gray), the matching procedure is emphasized.

\section{User variability}

For this part of the study, 13 patients scheduled for external beam radiotherapy for prostate cancer provided their written informed consent to acquire transabdominal US scans during simulation and every other treatment day [approved by the Internal Review Board (IRB)]. All patients were asked to follow a strict bladder filling protocol, i.e., they were asked to empty their bladder $1 \mathrm{~h}$ before treatment or simulation, followed by drinking 0.5 liter water within half an hour.

Figure 2 shows the setup of the patient study. For each patient, at four separate days during the treatment, one operator made two US scans directly in succession to assess the intra-operator scanning variability. At four other treatment fractions, two operators both made one US scan directly after each other to determine the inter-operator scan variabil- 
ity. All scanning operators received thorough training and strict scanning instructions. For each patient all repeated scans are matched by one single operator using the RPV.

The influence of the probe pressure on the prostate location was verified by US scans by one operator acquiring two scans at four different pressure levels. The probe was positioned at the level of the superior bladder dome and pressure was exerted in the posterior direction. The first level of pressure, no pressure, was acquired with the probe just touching the skin. At the second level, low pressure, the probe was placed on the skin, resting with its own weight on the abdomen. For the third level (intermediate pressure), the probe was pushed downwards with some pressure. The last level (high pressure) had the probe pushing downwards with pressure close to patient tolerance. The skin displacement, used in lieu of the applied pressure, was measured approximately with the room lasers projecting on a ruler attached to the probe for the occasion (the no pressure level was defined as $0 \mathrm{~mm}$, the low pressure level appeared to be $\sim 10 \mathrm{~mm}$, intermediate pressure $\approx 20-30 \mathrm{~mm}$ and high pressure $\approx 40 \mathrm{~mm}$ ). During normal use the probe pressure approaches closely the low pressure mode. The prostate displacement, defined as the average difference from two measurements normalized to the no pressure level, was determined during the matching procedure.

On the remaining days with single scans, the focus is on the RPV matching procedure. To emphasize the matching reproducibility of the operators separately (intra-operator variability), per patient one scan is randomly selected and matched five times by the same operator. This is repeated by a second operator. Finally, all the single scans are matched by three operators to focus on the matching difference between the operators (interoperator variability).

\section{Comparison of prostate localization}

In addition to the 13 patients mentioned before, another 8 patients scheduled for external beam radiotherapy for prostate cancer gave their informed consent to participate in the second phase of the IRB approved evaluation study for prostate localization.

All 8 patients got 3 or 4 gold FMs implanted $(1 \times 5 \mathrm{~mm}$ cylindrical gold wire) and underwent daily prostate localization using both transabdominal US and MV EPI with FMs, resulting in 244 combined US-EPI scans.

The normal clinical simulation process was combined with the Clarity workflow and the treatment planning (XiO CMS 4.51, Elekta, Stockholm, Sweden) was performed using the CT-scan. Two orthogonal digitally reconstructed radiographs (DRRs) were created per patient and the FMs were delineated manually on the DRRs for the standard FM matching procedure.

During treatment the patient was positioned using the room lasers and initial shifts were executed to place the target approximately in the beam center. The US scans were acquired directly followed by the MV EPI (Optivue AN9, Siemens, Germany, $10 \times 10 \mathrm{~cm}$ field @6 MV , 3 monitor units per beam) to minimize patient motion in-between the two measurements. If necessary a correction shift was performed based on the FM matching procedure (Theraview-NT 2.4, Cablon Medical, Leusden, the Netherlands). 


\section{Data analysis and statistics}

The standard deviation (SD) of the directional difference in prostate placement between two US scans focused on the scanning procedure was used to measure the intra- and inter-operator scanning aspects of the user variability of the US system. To obtain the difference in placement, per patient both scans were matched by one single user. The differences were averaged per patient and the SD was calculated. The final measure for the inter- and intra-operator scanning variability is the average of the SD over all patients.

For the matching variability, the SD of the five measurements was calculated per patient per operator and averaged to obtain the overall intra-operator matching variability. For the inter-operator variability, an average of 15 scans per patient was matched by three operators. Per scan the SD of the three measurements was calculated and averaged per patient. The final inter-operator matching variability is the average SD over all 13 patients. Because the measurements of the scanning variability are partially dependent on the matching procedure, and vice versa, both variabilities could not be completely separated. For the probe pressure analysis, the prostate displacement obtained from the US image (the average difference from two measurements normalized to the no pressure level) is per patient plotted against the skin displacement, measured by the ruler.

The difference between MV EPI and US prostate localization is depicted in a correlation plot, a Bland-Altman plot, ${ }^{32}$ and a histogram. The correlation plot contains a regression line and the coefficient of determination $\left(R^{2}\right)$. In the Bland-Altman plot, the limits of agreement (LOA) (bias $\pm 1.96 * \mathrm{SD}$ ) cover $95 \%$ of the measurements and are a measure whether the two methods are interchangeable. If the two limits are larger than a clinically relevant difference one would accept that changing the method to treat the patient will cause a relevant difference. Which method is the better can only be determined when both are compared to the absolute truth. When there is no absolute truth to compare to, only the difference between two methods can be measured. This is the case in our study. The confidence intervals $\left(\mathrm{Cl} ; \pm 1.96 *\right.$ error, error mean $=\mathrm{V}\left(\mathrm{s}^{2} / \mathrm{n}\right)$, error erD $\left._{\mathrm{S}}=\mathrm{V}\left(3 \mathrm{~s}^{2} / \mathrm{n}\right)\right)$ describe the uncertainty in the calculated values. The $95 \%$ LoA depends on the assumption that the differences are from an approximately normal distribution; frequency histograms are plotted to check if this assumption is valid.

\section{Speed of sound corrections in US imaging}

Quantitative US scanning may require an image distortion correction when tissues are imaged which have a SOS different than the standard value of $1540 \mathrm{~m} / \mathrm{s}$ employed by most US systems. ${ }^{24-27}$ Recently, a correction procedure was developed by us, and its clinical use was studied extensively. ${ }^{25-27}$ However, we need to emphasize that in our study, an intramodality US system is used to compare the position of the prostate on two different US images to derive a correction shift. Therefore, the SOS aberration is present in both images and the difference between the two is a second order effect. Consequently its influence on the shift is negligible. In contrast, in intermodality procedures (which were used in nearly all the older studies ${ }^{15,17}$ ) these SOS induced aberrations will cause, depending on the details of the workflow, a misinterpretation of the prostate location. ${ }^{26,27}$ Therefore, it is interesting to estimate the magnitude of the SOS aberration, even though it has 
little importance for the present study. To this end, the reference US $\mathrm{S}_{\text {sim }}$ and CT-scans of the eight patients of the US EPI comparison study were used for SOS error calculations according to the algorithm developed by us. ${ }^{25}$ The coregistered USsim- and CT-scans were exported from Clarity and imported in Osirix (Pixmeo SARL, Switzerland). The 3D volumes were re-sliced along the line of view of the US-probe through the middle of the prostate. Both the CT and $\mathrm{US}_{\text {sim }}$ midprostate slices are exported and used for MATLAB (Math-Works Inc., Natick, MA) calculations. A delineation of the prostate on the CT slice is used to define the region of interest of which the SOS distortion of its centroid is assessed.

\section{RESULTS}

\section{User variability}

The results of the user variability for scanning and matching are shown in Table 1 . As expected, for both operator steps the intra-operator variability is smaller than the interoperator one. Furthermore, the match variability is smaller than the variability for scanning. This is because both variability values could not be measured completely independently, and the matching component is more prominently present in the scanning analysis than vice versa. (To determine the scanning variability values, each image had to be matched. To be able to match the US images multiple times, one of the users had to acquire the scans.)

The prostate displacement due to the probe pressure varies from patient to patient and is, in our patient population, not limited to one direction (Figure 3 ). In some patients even a shift opposite to the probe pressure direction was observed.

The total displacement vector increases when more pressure is applied, although looking at the complete patient population, no obvious trend in prostate displacement was observed. The average displacement vector is about $3 \mathrm{~mm}$ for a $1 \mathrm{~cm}$ skin displacement. With our bladder filling protocol, low pressure was in most cases sufficient to obtain good image quality. At low pressure the mean prostate displacement was $-0.5 \mathrm{~mm}$ right, $0.7 \mathrm{~mm}$ posterior, and $0.0 \mathrm{~mm}$ superior. 
$80 \mid$ Chapter 4
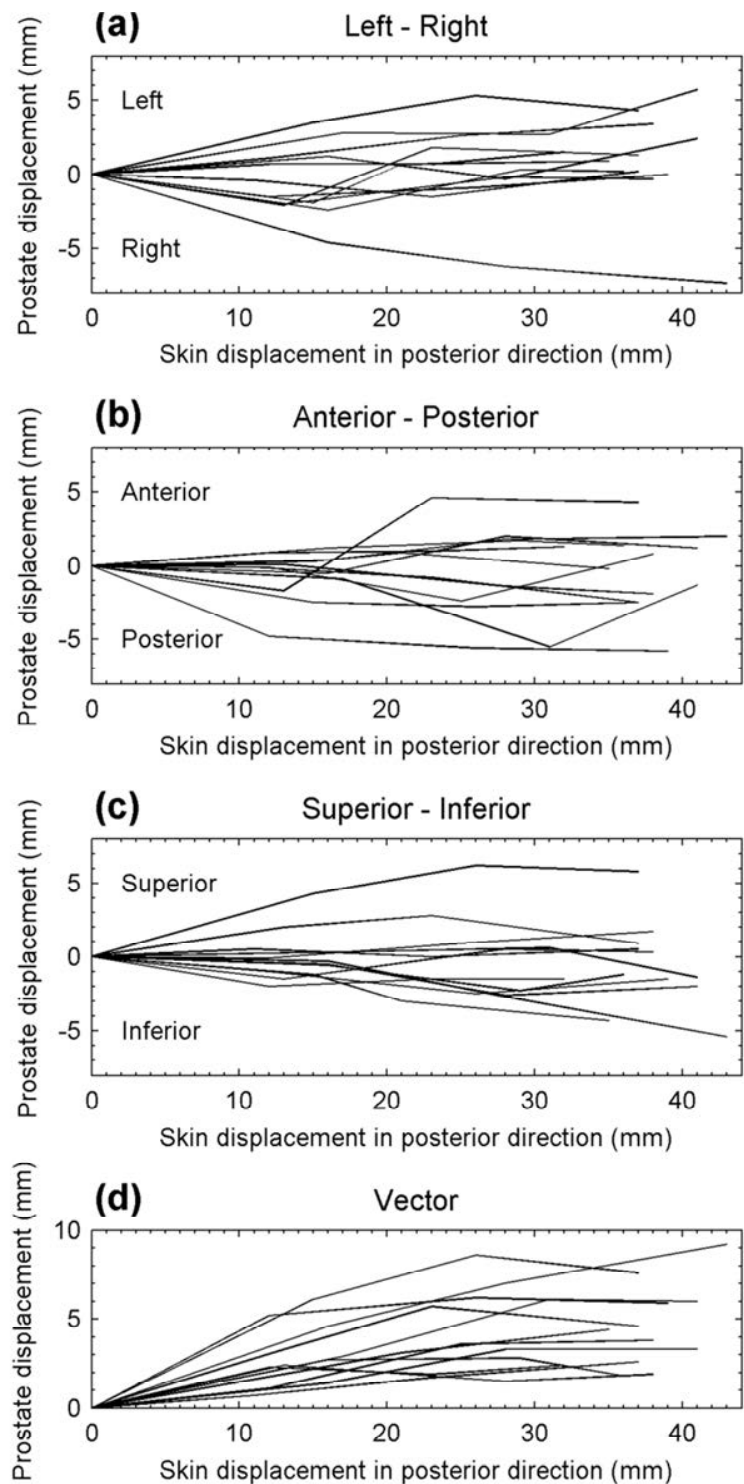

Figure 3: Prostate motion due to probe pressure. The US prostate displacement (the average difference from two measurements normalized to the no pressure level) plotted against the skin displacement for each direction (a)-(c) and for the total displacement vector (d). Each line represents a different patient. 
Table 1. The user variability for the US localization procedure differentiated for different operator aspects: Inter- and intra-operator variability for both the scanning and matching procedures.

\begin{tabular}{lccc}
\hline & Left-Right $(\mathrm{mm})$ & Anterior-Posterior $(\mathrm{mm})$ & Superior-Inferior $(\mathrm{mm})$ \\
\hline Scan variability (SD) & & & \\
Intra-operator & 1.8 & 2.2 & 2.7 \\
Inter-operator & 2.6 & 2.2 & 2.9 \\
Match variability (SD) & & & \\
Intra-operator & 0.8 & 0.7 & 1.0 \\
Inter-operator & 1.3 & 1.4 & 1.8 \\
\hline
\end{tabular}

\section{Comparison of prostate localization from US and MV EPI FM imaging}

The differences between MV EPI and US prostate localization range in the left-right (LR) direction from -6.6 to $16.9 \mathrm{~mm}$, in the superior-inferior (SI) direction from -17.9 to $9.7 \mathrm{~mm}$ and in the anterior-posterior (AP) direction from -14.3 to $7.9 \mathrm{~mm}$. For the $\mathrm{LR}, \mathrm{SI}$, and AP direction, respectively, $74 \%, 73 \%$, and $77 \%$ of the measured differences are below $5 \mathrm{~mm}$. In $56 \%$ at least one of the directions has a difference above $5 \mathrm{~mm}$ (for $10 \mathrm{~mm}$, this is $11 \%$ ). The average differences $\pm \mathrm{SD}$ are: $2.5 \pm 4.0 \mathrm{~mm}(\mathrm{LR} ; \mathrm{Cl}: 2.0-3.0 \mathrm{~mm}), 0.6 \pm 4.9 \mathrm{~mm}(\mathrm{Sl} ; \mathrm{Cl}$ : 0.0-1.2 mm), and $-2.3 \pm 3.6 \mathrm{~mm}$ (AP; Cl: $-2.7--1.8 \mathrm{~mm}$ ). This leads to LoA of $-5.4-10.3 \mathrm{~mm}$ [LR, Figure 4(b); $\mathrm{Cl}_{\text {LowerLimit: }}$-6.2--4.5 mm, Cl UpperLimit: 9.5-11.2 mm], -8.9-10.2 mm [SI, Figure 4(e); $\mathrm{Cl}_{\text {LowerLimit: }}$-10.0--7.8 mm, Cl UpperLimit: 9.1-11.2 mm], and -9.3-4.7 mm [AP, Figure 4(h); $\mathrm{Cl}_{\text {LowerLimit: }}$-10.0--8.5 mm, Cl UpperLimit: 3.9-5.5 mm]. For the LR direction, there is no correlation $\left(R^{2}=0.06\right)$ between the two localization methods [Figure $4(a)$ ]. In both the $\mathrm{SI}$ and AP direction, there is a moderate correlation $\left[S I: R^{2}=0.57\right.$, Figure 4(d); AP: $R^{2}=$ 0.77 , Figure $4(\mathrm{~g})]$. The frequency histograms of the differences follow approximately a normal distribution.

\section{Speed of sound corrections in US imaging}

An indication of the SOS aberration is given in Table 2. In all patients the correction shift has a negative value, which means that the real prostate position is towards the US transducer. On the US images the prostate appears to be deeper inside the abdomen than it is in reality. Note that the mentioned SOS distortions are only an indication of the prostate shift along an angled plane, and not based on a 3D calculation of the complete prostate shift/deformation. Furthermore, due to the intramodality system, the SOS influence on the matching results is negligible. ${ }^{26}$ 


\section{Correlation US-FM}

(a) Left -- Right

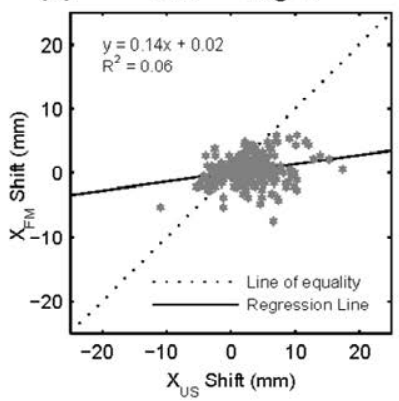

(d) Superior -- Inferior

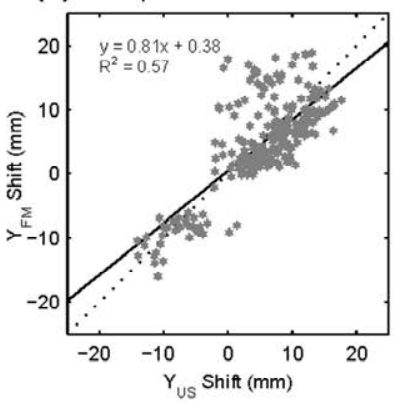

(g) Anterior-- Posterior

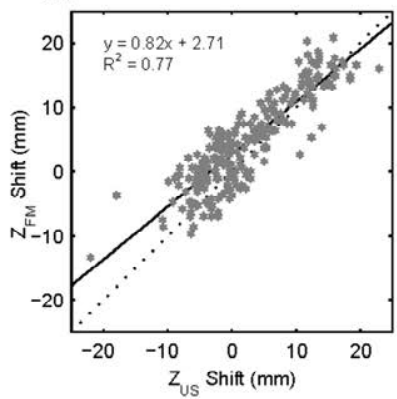

Bland-Altman plot US-FM

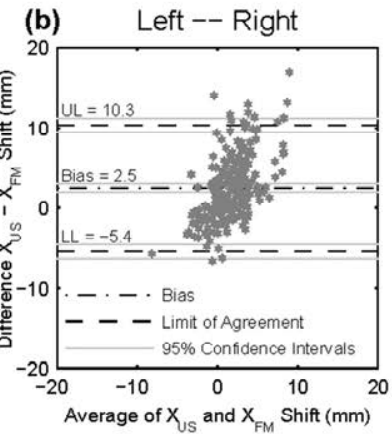

(e) Superior -- Inferior

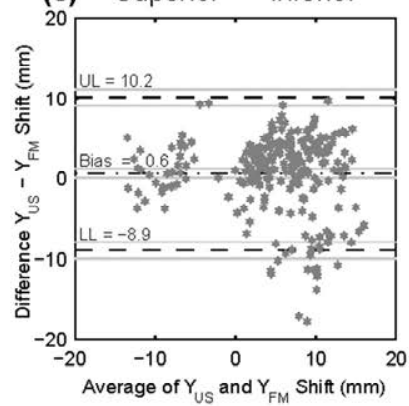

(h) Anterior-- Posterior

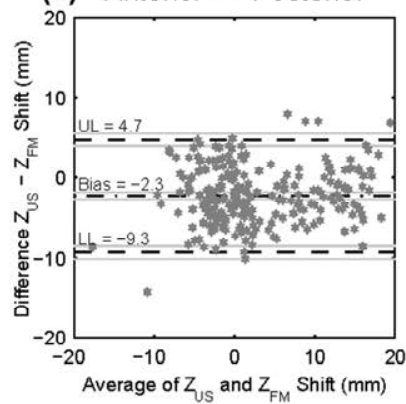

Histogram

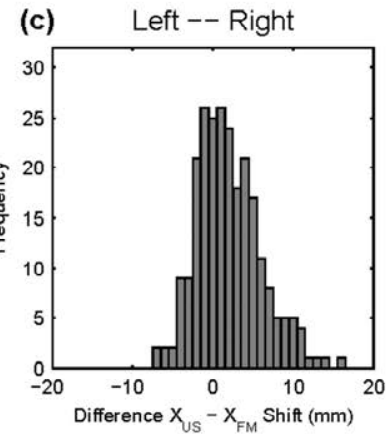

(f) Superior -- Inferior

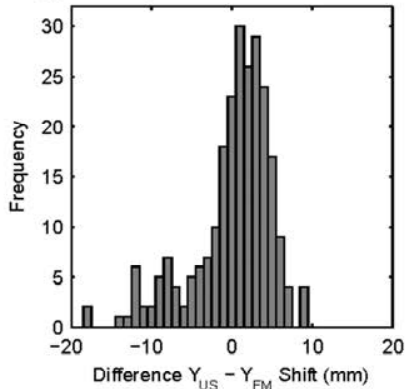

(i) Anterior-- Posterior

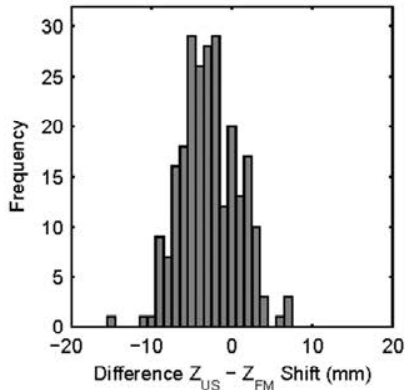

Figure 4: Shifts measured with US localization vs FM MV portal image-based shifts shown in correlation plots $(a, d, g)$, Bland-Altman analysis $(b, e, h)$, and their accompanying histograms $(c, f, i)$. (Abbreviations: UL, upper limit; $L$, lower limit). 
Table 2. Estimate of the SOS distortion of the prostate centroid along the central line of view of the US-probe. Negative values indicate that the true prostate position is shallower than is shown in the US images.

\begin{tabular}{cc}
\hline Patient & SOS shift (mm) \\
\hline 1 & -2.9 \\
2 & -3.5 \\
3 & -3.7 \\
4 & -4.0 \\
5 & -3.3 \\
6 & -2.2 \\
7 & -3.7 \\
8 & -2.2 \\
Mean \pm SD & $-3.2 \pm 0.7$ \\
\hline
\end{tabular}

\section{DISCUSSION}

\section{Accuracy issues}

Several publications comparing 2DUS and FM-based prostate localization methods have raised concerns regarding the accuracy of US-based techniques. ${ }^{33-35}$ The main concerns are about US user variability and abdominal pressure ${ }^{21-23}$ causing organ motion. In the first part of this study the user variability of the 3DUS system was investigated. The US matching has a comparable procedure and influence as in manual FM alignment in EPI images. Indeed, the intra-operator match variability of US $(0.7-1.0 \mathrm{~mm})$ is similar to the FM intraoperator variability $(0.4-1.0 \mathrm{~mm})$ in the study of Ullman et al. ${ }^{36}$ Nonetheless, the interoperator match variability of US $(1.3-1.8 \mathrm{~mm})$ is considerably larger than that of FMs $(0.7$ $\mathrm{mm}$ ) in their study ${ }^{36}$ and that of Kong et al. ${ }^{37}$ (SI: $0.6 \mathrm{~mm}, \mathrm{AP}: 0.7 \mathrm{~mm}$ ).

The image acquisition stage is not user dependent for MV EPI while for the US system it is. The user variability determined for US scanning was $2.2-2.9 \mathrm{~mm}$. Even though the focus was on the scanning procedure, the complete image guidance workflow had to be followed. Since every scan also had to be matched, the scan variability also contains the matching variability. Therefore, the mean value of the scan variability determined in this study is actually the overall user variability of the complete process.

Summarizing the uncertainties of the complete workflow of both methods as used in our clinic, it was found that the combined uncertainties of both methods are similar (Table 3). In this study, the delineation of the RPV for the US system was not under investigation. Nevertheless, as long as this delineation is only used as RPV and not as treatment target, the uncertainty of the RPV is comparable to the uncertainty on the manual delineation of the FMs on the DRR. Neither were investigated in this study and are therefore not included in Table 3. 
Table 3. Overview of the uncertainties (1 SD) of the 3DUS system and (manual) FM positioning on MV EPI.

\begin{tabular}{lllll}
\hline \multicolumn{2}{l}{ 3DUS } & \multicolumn{3}{c}{ MV EPI with FMs } \\
\hline US-Sim $^{\mathrm{a}}$ & $1.0 \mathrm{~mm}$ & CT & $1.5 \mathrm{~mm}$ & $1 / 2$ CT slice thickness \\
US-Guide $^{\mathrm{a}}$ & $1.0 \mathrm{~mm}$ & EPI & $0.4 \mathrm{~mm}$ & EPI resolution \\
User variability & $2.6 \mathrm{~mm}$ & Migration & $0.6 \& 0.5 \mathrm{~mm}$ & $\Sigma \& \sigma\left(\right.$ Ref. $\left.^{38}\right)$ \\
& & Deformation & $0.4 \& 1.1 \mathrm{~mm}$ & $\Sigma \& \sigma$ (Ref. $^{39}$ ) \\
& & User variability & $0.7 \mathrm{~mm}$ & Refs. $^{36}$ and $^{37}$ \\
Total & $3.0 \mathrm{~mm}$ & Total & $2.2 \mathrm{~mm}$ & \\
\hline
\end{tabular}

Abbreviations: $\Sigma$, systematic error; $\sigma$, random error.

${ }^{\mathrm{a}}$ These are the uncertainties on which the manufacturer bases the QC action levels, as a reference: our average QC result was $0.74 \pm 0.57 \mathrm{~mm}$ (US-Sim and US-Guide combined).

Transducer pressure and SOS aberrations ${ }^{26}$ are also not in the uncertainty overview because with the use of an intramodality system these uncertainties are minimized when similar scanning procedures are used to acquire the $U S_{\text {Sim }}$ and $U S_{t x}$ images. This is because we derive corrective shifts as differences between two images, both affected by the SOS aberration and the transducer pressure. Due to strict bladder filling protocols, similar probe placement and low probe pressure between $\mathrm{US}_{\mathrm{Sim}}$ and $\mathrm{US}_{\mathrm{tx}}$, the SOS artifact and movement due to pressure will be comparable during simulation and treatment. In the literature sometimes large prostate displacements are reported due to US probe pressure. $^{21-23}$ This is probably due to excessive pressure on the patient's skin which, in particular in radiology, is believed to be necessary to acquire high quality images. For US IGRT this is not needed in our experience and, as stated, for intramodality US systems the effect is mitigated. Because the US $\mathrm{S}_{\mathrm{Sim}}$ and $\mathrm{CT}$ images are made in the same position and reference frame, the automatic fusion will neutralize the SOS artifact and pressure movement in the US scan. This is not the case for intermodality systems where a treatment room US image is compared to a reference CT image, nor when manual fusion is per-

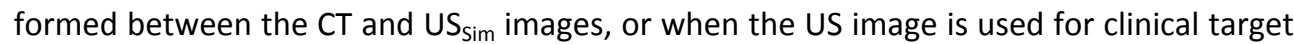
volume delineation. Nevertheless, pressure should always be minimized during US scanning to avoid problems with reproducibility. The applied probe pressure in this study was, with exception of the pressure test, always low pressure, i.e., the probe was resting with its own weight on the abdomen. This led to an adequate imaging quality to locate the prostate and minimal prostate displacement. In the pressure test, the observed prostate shift opposite to the probe pressure direction can partially be explained by the fact that the pressure was applied to the bladder and not directly to the prostate itself. Another reason is that when the pressure becomes high, patients may respond by motion or muscle contraction to provide some counter pressure.

Besides proper training, improvements on the US system can be gained by automating the imaging system to avoid user dependency. For instance, recently developed robot-arm held US-probes ${ }^{40}$ may decrease the scanning variability, and automated prostate detection can improve the matching procedure. Also for the FM matching, automatic marker detection can improve the FM accuracy. ${ }^{41,42}$ 


\section{Comparison of the two IGRT techniques}

Focusing on the localization comparison of US and FMs, there seems to be a clinically relevant difference ( $>5 \mathrm{~mm}$ in at least one direction) in $56 \%$ of the patients (for $10 \mathrm{~mm}$, this is $11 \%$ ). The average difference between the two methods exceeds $2 \mathrm{~mm}$ in the LR and AP directions, while for the SI direction it remains below $1 \mathrm{~mm}$. Remarkably, in particular in the LR direction, the detected shifts are larger using US than with the use of FMs. In general it is commonly assumed that the prostate motion in the lateral direction is minimal. This may lead to the conclusion that, in the lateral direction, the US shift results are unreliable. However, other studies with electromagnetic transponders, ${ }^{10} \mathrm{CBCT}$, and $\mathrm{FMs}{ }^{43}$ report a lateral shift that is comparable to our US results. Nonetheless, a lower visibility of the lateral boundary in US images could also explain the difference between US and the FMs, although this didn't translate into a larger LR user variability than in the other directions. Patient motion in-between the acquisition of the two image modalities or intrafractional prostate motion ${ }^{44}(-0.1 \mathrm{~mm}(\mathrm{LR}), 0.2 \mathrm{~mm}(\mathrm{SI})$, and $2.3 \mathrm{~mm}(\mathrm{AP})$ ) could result in this difference in shifts. However, the time between the two scan was minimalized and the acquisition was repeated when patient motion was detected or suspected.

Another issue is the SOS aberration. Although playing only a minimal role in this work due the intramodality character of the Clarity system, it almost certainly introduced systematic errors in the older studies comparing intermodality US systems against other imaging modalities. It is striking that the reported SOS corrections for US prostate imaging always result in a ventral shift of the organ. We have reported before that this correction would result in a reduced discrepancy between US and other imaging modalities, even to the extent that most of the discrepancy could be explained by the SOS aberration alone. ${ }^{27}$

For all three directions one of the LoA is close to $1 \mathrm{~cm}$ to cover $95 \%$ of the measurements (LR: $10.3 \mathrm{~mm}$, SI: $10.2 \mathrm{~mm}$, AP: $-9.3 \mathrm{~mm}$ ). This implies that FM based prostate localization is not heedlessly interchangeable with US, as it will lead to an altered treatment setup. In particular since the planning target volume margins are between 8 and 11 $\mathrm{mm}$. However, since neither method can be considered to be the absolute truth, this does not imply that prostate positioning with US imaging is worse than FM based surrogate imaging. A study ${ }^{45}$ comparing soft tissue imaging with $\mathrm{kV}$ CBCT vs FM MV EPI reported LoA of $9 \mathrm{~mm}$ (LR: -4.1-3.9 mm, SI: -9.0-5.3 mm, AP: -4.0-9.3 mm), comparable to the value of about $10 \mathrm{~mm}$ we obtained (in their paper the LoA are reported as the confidence intervals ${ }^{46}$ ). Another group ${ }^{47}$ reported LoA up to $6 \mathrm{~mm}$ between $\mathrm{kV}$ CBCT vs FM MV EPI, which is smaller, but can still be considered clinically relevant.

With the FM MV EPI method, metal markers are imaged as surrogates of the prostate in two 2D projections. In the case of prostate rotations and deformations, the difference in centroid position of the RPV and FMs and the coverage of the prostate volume will result in different correction shifts. (In extreme cases a $2 \mathrm{~mm}$ difference for a $7^{\circ}$ prostate rotation and a distance between centroids of $16.5 \mathrm{~mm}$.) The geometrical characteristics of the prostate CT contour, the RPV, and the FMs are, per patient, shown in Table 4 and Figure 5. In all patients, the equivalent radius of the prostate CT contour is the largest, ${ }^{48-50}$ followed by the equivalent radius of the RPV and then that of the FMs. In seven of the eight patients the distance between the centroids of the RPV and the prostate is smaller than that of the FM and the prostate. Besides, independent manual processing of the FM 
in the orthogonal DRR 2D projections may lead to ambiguities in the correction of the patient. For example, FM rotations visible in one plane will cause differences between the inter-FM distances in the perpendicular plane. Being a surrogate for the prostate position, FM migration and prostate deformation can lead to misinterpretation of the prostate location. FMs have also been reported to migrate away from the implantation site. ${ }^{1,51}$ In $84 \%$ of the patients, the maximum difference in intermarker distance was at least $2 \mathrm{~mm} .{ }^{52}$ The corresponding numbers for 3, 4, and $5 \mathrm{~mm}$ were $41 \%, 18 \%$, and $9 \%$ of the patients, respectively. ${ }^{52}$ Because most of our patients got their planning CT within a week after FM implantation, the location of the FMs might not have been completely stable. The Clarity US-system images the prostate directly in 3D thus avoiding these drawbacks. There have also been reports on non-rigid prostate deformations and prostate volume changes over time $^{53-56}$ In these cases dose recalculation may be required to assess the accumulated dose accurately.

Table 4: Overview of the patient specific geometrical characteristics of the prostate CT contour (PCTC), the RPV and the FMs.

\begin{tabular}{lccccccccc}
\hline Patient & $\begin{array}{c}\text { Volume } \\
\text { (cc) }\end{array}$ & \multicolumn{3}{c}{$\begin{array}{c}\text { Equivalent radius* } \\
\text { (mm) }\end{array}$} & \multicolumn{2}{c}{$\begin{array}{c}\text { Distance between centroids } \\
\text { (mm) }\end{array}$} & $\begin{array}{c}\text { Difference US-FMs } \\
\text { shift (mm) }\end{array}$ \\
\hline 1 & PCTC & RPV & PCTC & RPV & FMs & PCTC-RPV & PCTC-FMs & RPV-FMs & Average vector \\
\hline 2 & 69.9 & 53.9 & 25.5 & 23.4 & 16.9 & 4.0 & 7.5 & 8.7 & 11.0 \\
3 & 36.9 & 19.5 & 20.7 & 16.7 & 11.1 & 3.6 & 5.4 & 5.8 & 5.9 \\
4 & 39.2 & 16.2 & 21.1 & 15.7 & 9.3 & 5.1 & 13.8 & 15.3 & 9.9 \\
5 & 45.0 & 33.2 & 22.1 & 19.9 & 13.4 & 3.8 & 14.0 & 16.5 & 4.5 \\
6 & 82.1 & 56.8 & 27.0 & 23.9 & 12.8 & 3.7 & 8.1 & 8.9 & 5.3 \\
7 & 102.3 & 76.9 & 29.0 & 26.4 & 22.8 & 3.9 & 3.0 & 6.9 & 5.7 \\
8 & 68.0 & 36.5 & 25.3 & 20.6 & 12.9 & 3.0 & 4.9 & 7.3 & 8.3 \\
\hline
\end{tabular}

* The equivalent radius for the FMs is the average distance between the markers and their centroid. The equivalent radius for the prostate CT contour and the RPV is the radius of a sphere with the same volume as the prostate CT contour/RPV.

FM implantation is not always possible in all prostate cancer patients, e.g., due to hemorrhagic and/or infection risks, leading in extreme cases to a complication rate of $20 \%{ }^{4,5}$ Known dose delivery inaccuracies occur due to the presence of metal bodies and their concomitant streaking artifacts in CT images in case of photon or proton beam treatments $^{7-9}$ or combinations with low dose rate (LDR) brachytherapy. ${ }^{6}$ The presence of the FMs can cause localized dose reductions between the calculated and delivered dose for proton therapy of up to $38 \%$ (Ref. ${ }^{7}$ ) or $47 \%{ }^{8}$ depending on the marker and the reference consulted. For high energy photon beam therapy the relative dose at the upstream (downstream) seed surface was calculated to be up to $64 \%$ higher ( $12 \%$ lower) for a single $6 \mathrm{MV}$ parallel beam. ${ }^{9}$ In the case where LDR brachytherapy is used as a boost after external beam radiotherapy the dose perturbation can be quite severe with localized brachytherapy dose reductions of $50 \%{ }^{6}$ 
But can US replace the FM workflow? Johnston et al. ${ }^{16}$ used an earlier research version of the Clarity system with a different workflow than in our study, and they report slightly larger variation between US and FM with a similar number of patients. They state that US cannot safely replace FMs. In their conclusion, they accept the FM procedure as the standard without assessing its uncertainties. In our opinion, the systematic differences between the two methods are not automatically indicating that the FM workflow is more accurate than US. Yet, more recently Robinson et al. ${ }^{19}$ also found the Clarity system, with the same workflow as our system, incapable of safely replacing FMs. In their study with 17 patients, the US system was compared to CT. However, they did not use the complete recommended US workflow; all the US images were acquired in the simulation room and individually delineated. This clearly deviates from the normal clinical workflow. The recommended workflow of the Clarity system is that the initial US contour from the first US is copied to all following US images and manually shifted until it fits the edges of the current ultrasound image. Also the automatic adapt function ${ }^{30,31}$ which is present at the US unit in the treatment room should be applied. Possible re-delineation inaccuracies, which are known to be the largest contributor to the variability, are not present in the normal workflow.

In a very recent study by Mayyas et al., ${ }^{20}$ with 27 patients, the Clarity US system gave similar positioning results in comparison to daily imaging with $\mathrm{kV} x$-rays, $\mathrm{CBCT}$, and electromagnetic transponders. The conclusion from our own results is that the accuracy of US is comparable to our FM workflow. A limitation of our study (and most older studies) is the small number of patients. We do not expect, however, any of our conclusions to be altered much by performing a larger patient study. We cannot exclude, though, that a few outliers may have influenced some of our results (Figure 4 does not suggest severe outliers).

The effects on the day-to-day workflow, if one would opt for US IGRT, are minimal. No extra physician involvement is necessary; all US images are acquired by the radiotherapy technologists. Time and staff can even be saved because of the redundancy of the FM implantation. The time necessary to accomplish the complete US workflow in the treatment room (acquire the $\mathrm{US}_{\mathrm{tx}}$, matching to $\mathrm{US}_{\text {sim }}$, and shifting the patient) is, after proper training, less than $3 \mathrm{~min}$.

Because of a better prostate volume representation, a minimally increased treatment time, avoidance of FM implantation (removing the risk of bleeding and prostate inflammation), and avoidance of extra radiation burden prostate localization with US imaging is a reasonable alternative for patients for whom FM MV EPI positioning is not desirable. In the clinical decision which positioning technique to choose, the treatment margins should also be taken into account. Patient preference towards a noninvasive procedure may also guide the choice between the two methods. Our study has shown though, that for $56 \%$ (decision criterion of $5 \mathrm{~mm}$; for $10 \mathrm{~mm}$, this is 11\%) of patients US imaging may lead to significantly different shifts in at least one direction compared to FM EPI imaging, which does not necessarily mean that EPI results are more reliable, in view of the reported uncertainties in this study. 


\section{Patient 1}

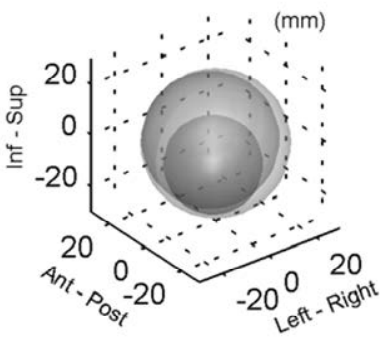

Patient 3

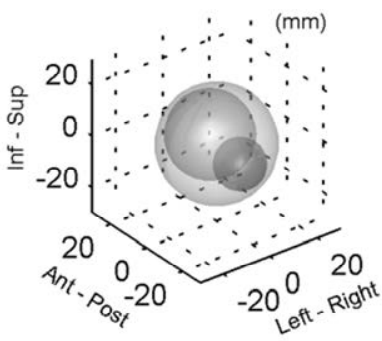

Patient 5

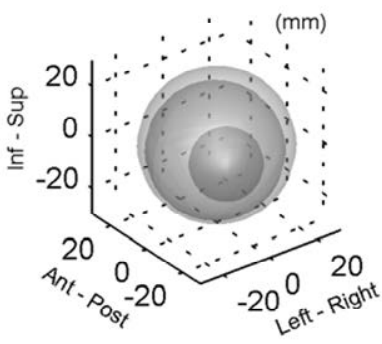

Patient 7

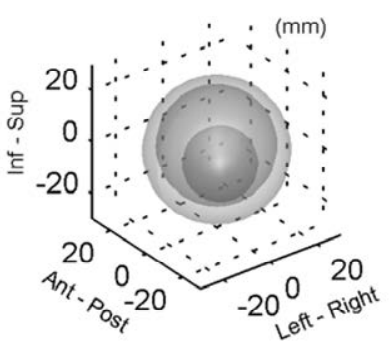

Patient 2

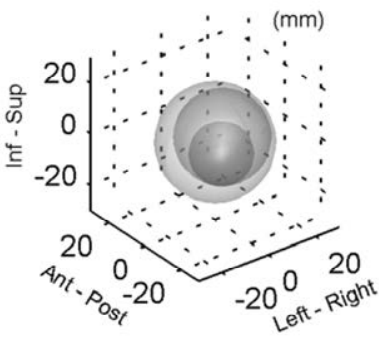

Patient 4

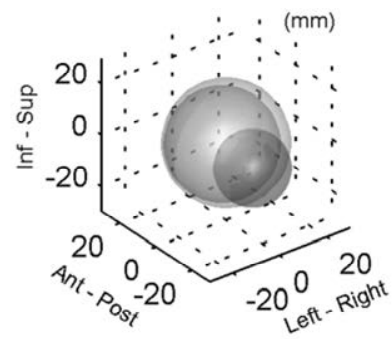

Patient 6

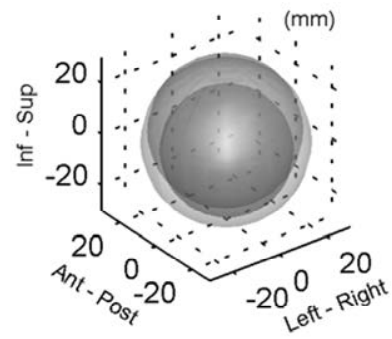

Patient 8

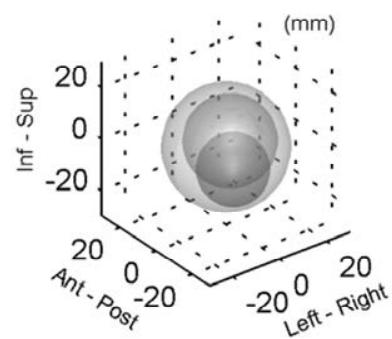

Figure 5: Graphical overview of the patient specific geometrical characteristics of the prostate CT contour (PCTC), the RPV, and the FMs. The large, light gray spheres have a radius equal to the equivalent radius of the PCTC. Using the radius and relative centroid positions from Table 4, the medium sized and medium gray spheres represent the coverage of the PCTC by the RPV and the small, dark gray spheres represent the PCTC coverage by the FMs. 


\section{ACKNOWLEDGMENTS}

The authors like to thank the patients who agreed to participate in the study and the therapy staff at the treatment units. The authors gratefully acknowledge Elekta for their technical support of this work. Comments by Dr M. Lachaine from Elekta on the work are gratefully acknowledged. The authors also would like to thank Dr. L. Beaulieu for his exchange of views on FM-based prostate localization and Dr. Enrica Seravalli and Dr. L. Murrer for their help with the planning of part of the study. The authors would like to thank R. Houben for his help with the statistics. SvdM is in part supported by GROW (School for Oncology and Developmental Biology, Maastricht University). The authors report no conflicts of interest in conducting the research.

\section{REFERENCES}

1 U.A. van der Heide, A.N. Kotte, H. Dehnad, et al., "Analysis of fiducial marker-based position verification in the external beam radiotherapy of patients with prostate cancer", 2007 Radiother Oncol (82:38-45)

2 A.M. Henry, C. Wilkinson, J.P. Wylie, et al., "Trans-perineal implantation of radio-opaque treatment verification markers into the prostate: an assessment of procedure related morbidity, patient acceptability and accuracy", 2004 Radiother Oncol (73:57-59)

3 J.F. Langenhuijsen, E.N. van Lin, L.A. Kiemeney, et al., "Ultrasound-guided transrectal implantation of gold markers for prostate localization during external beam radiotherapy: complication rate and risk factors", 2007 Int J Radiat Oncol Biol Phys (69:671-676)

4 S. Igdem, H. Akpinar, G. Alco, et al., "Implantation of fiducial markers for image guidance in prostate radiotherapy: patient-reported toxicity", 2009 Br J Radiol (82:941-945)

5 V. Fonteyne, P. Ost, G. Villeirs, et al., "Improving positioning in high-dose radiotherapy for prostate cancer: safety and visibility of frequently used gold fiducial markers", 2012 Int J Radiat Oncol Biol Phys (83:46-52)

6 G. Landry, B. Reniers, L. Lutgens, et al., "Dose reduction in LDR brachytherapy by implanted prostate gold fiducial markers", 2012 Med Phys (39:1410-1417)

7 J. Cheung, R.J. Kudchadker, X.R. Zhu, et al., "Dose perturbations and image artifacts caused by carboncoated ceramic and stainless steel fiducials used in proton therapy for prostate cancer", 2010 Phys Med Biol (55:7135-7147)

8 J.Y. Huang, W.D. Newhauser, X.R. Zhu, et al., "Investigation of dose perturbations and the radiographic visibility of potential fiducials for proton radiation therapy of the prostate", 2011 Phys Med Biol (56:52875302)

9 J.C. Chow, G.N. Grigorov, "Monte Carlo simulations of dose near a nonradioactive gold seed", 2006 Med Phys (33:4614-4621)

10 R.D. Foster, T.D. Solberg, H.S. Li, et al., "Comparison of transabdominal ultrasound and electromagnetic transponders for prostate localization", $2010 \mathrm{~J}$ Appl Clin Med Phys (11:2924)

11 L.M. Girouard, J. Pouliot, X. Maldague, A. Zaccarin, "Automatic setup deviation measurements with electronic portal images for pelvic fields", 1998 Med Phys (25:1180-1185)

12 M. Oldham, D. Letourneau, L. Watt, et al., "Cone-beam-CT guided radiation therapy: A model for on-line application", 2005 Radiother Oncol (75:271-278)

13 G.G. Zeng, T.S. McGowan, T.M. Larsen, et al., "Calcifications are potential surrogates for prostate localization in image-guided radiotherapy", 2008 Int J Radiat Oncol Biol Phys (72:963-966)

14 B.W. Raaymakers, J.J. Lagendijk, J. Overweg, et al., "Integrating a 1.5 T MRI scanner with a 6 MV accelerator: proof of concept", 2009 Phys Med Biol (54:N229-237)

15 A.Y. Fung, K.M. Ayyangar, D. Djajaputra, et al., "Ultrasound-based guidance of intensity-modulated radiation therapy", 2006 Med Dosim (31:20-29)

16 H. Johnston, M. Hilts, W. Beckham, E. Berthelet, "3D ultrasound for prostate localization in radiation therapy: a comparison with implanted fiducial markers", 2008 Med Phys (35:2403-2413)

17 J. Morr, T. DiPetrillo, J.S. Tsai, et al., "Implementation and utility of a daily ultrasound-based localization system with intensity-modulated radiotherapy for prostate cancer", 2002 Int J Radiat Oncol Biol Phys (53:1124-1129) 
18 D.J. Fraser, Y. Chen, E. Poon, et al., "Dosimetric consequences of misalignment and realignment in prostate 3DCRT using intramodality ultrasound image guidance", 2010 Med Phys (37:2787-2795)

19 D. Robinson, D. Liu, S. Steciw, et al., "An evaluation of the Clarity 3D ultrasound system for prostate localization", 2012 Journal of applied clinical medical physics / American College of Medical Physics $(13: 3753)$

20 E. Mayyas, I.J. Chetty, M. Chetvertkov, et al., "Evaluation of multiple image-based modalities for imageguided radiation therapy (IGRT) of prostate carcinoma: A prospective study", 2013 Medical physics (40:041707)

21 X. Artignan, M.H. Smitsmans, J.V. Lebesque, et al., "Online ultrasound image guidance for radiotherapy of prostate cancer: impact of image acquisition on prostate displacement", 2004 Int J Radiat Oncol Biol Phys (59:595-601)

22 J.P. McGahan, J. Ryu, M. Fogata, "Ultrasound probe pressure as a source of error in prostate localization for external beam radiotherapy", 2004 Int J Radiat Oncol Biol Phys (60:788-793)

23 B. Dobler, S. Mai, C. Ross, et al., "Evaluation of possible prostate displacement induced by pressure applied during transabdominal ultrasound image acquisition", 2006 Strahlenther Onkol (182:240-246)

24 B.J. Salter, B. Wang, M.W. Szegedi, et al., "Evaluation of alignment error due to a speed artifact in stereotactic ultrasound image guidance", 2008 Phys Med Biol (53:N437-445)

25 D. Fontanarosa, S. van der Meer, E. Harris, F. Verhaegen, "A CT based correction method for speed of sound aberration for ultrasound based image guided radiotherapy", 2011 Med Phys (38:2665-2673)

26 D. Fontanarosa, S. van der Meer, F. Verhaegen, "On the significance of density-induced speed of sound variations on US-guided radiotherapy", 2012 Med Phys (39:6316-6323)

27 D. Fontanarosa, S. van der Meer, E. Bloemen-van Gurp, et al., "Magnitude of speed of sound aberration corrections for ultrasound image guided radiotherapy for prostate and other anatomical sites", 2012 Med Phys (39:5286-5292)

28 F.L. Cury, G. Shenouda, L. Souhami, et al., "Ultrasound-based image guided radiotherapy for prostate cancer: comparison of cross-modality and intramodality methods for daily localization during external beam radiotherapy", 2006 Int J Radiat Oncol Biol Phys (66:1562-1567)

29 J.A. Molloy, G. Chan, A. Markovic, et al., "Quality assurance of U.S.-guided external beam radiotherapy for prostate cancer: report of AAPM Task Group 154", 2011 Med Phys (38:857-871)

30 F. Lathuiliere, V. Audet, T. Falco, M. Lachaine, United States Patent No. 20070167699 A1 (19 July 2007).

31 N. Hu, D.B. Downey, A. Fenster, H.M. Ladak, "Prostate boundary segmentation from 3D ultrasound images", 2003 Medical physics (30:1648-1659)

32 J.M. Bland, D.G. Altman, "Statistical methods for assessing agreement between two methods of clinical measurement", 1986 Lancet (1:307-310)

33 F. Van den Heuvel, T. Powell, E. Seppi, et al., "Independent verification of ultrasound based image-guided radiation treatment, using electronic portal imaging and implanted gold markers", 2003 Med Phys (30:2878-2887)

34 K.M. Langen, J. Pouliot, C. Anezinos, et al., "Evaluation of ultrasound-based prostate localization for imageguided radiotherapy", 2003 Int J Radiat Oncol Biol Phys (57:635-644)

35 T.J. Scarbrough, N.M. Golden, J.Y. Ting, et al., "Comparison of ultrasound and implanted seed marker prostate localization methods: Implications for image-guided radiotherapy", 2006 Int J Radiat Oncol Biol Phys (65:378-387)

36 K.L. Ullman, H. Ning, R.C. Susil, et al., "Intra- and inter-radiation therapist reproducibility of daily isocenter verification using prostatic fiducial markers", 2006 Radiat Oncol (1:2)

37 V. Kong, G. Lockwood, J. Yan, et al., "The effect of registration surrogate and patient factors on the interobserver variability of electronic portal image guidance during prostate radiotherapy", 2011 Med Dosim)

38 H. Dehnad, A.J. Nederveen, U.A. van der Heide, et al., "Clinical feasibility study for the use of implanted gold seeds in the prostate as reliable positioning markers during megavoltage irradiation", 2003 Radiother Oncol (67:295-302)

39 A.M. Nichol, K.K. Brock, G.A. Lockwood, et al., "A magnetic resonance imaging study of prostate deformation relative to implanted gold fiducial markers", 2007 Int J Radiat Oncol Biol Phys (67:48-56)

40 J. Schlosser, K. Salisbury, D. Hristov, "Telerobotic system concept for real-time soft-tissue imaging during radiotherapy beam delivery", 2010 Med Phys (37:6357-6367)

41 S. Aubin, L. Beaulieu, S. Pouliot, et al., "Robustness and precision of an automatic marker detection algorithm for online prostate daily targeting using a standard V-EPID", 2003 Med Phys (30:1825-1832) 
42 A.J. Nederveen, J.J. Lagendijk, P. Hofman, "Feasibility of automatic marker detection with an a-Si flat-panel imager", 2001 Phys Med Biol (46:1219-1230)

43 O. Gayou, M. Miften, "Comparison of mega-voltage cone-beam computed tomography prostate localization with online ultrasound and fiducial markers methods", 2008 Med Phys (35:531-538)

44 T. Budiharto, P. Slagmolen, K. Haustermans, et al., "Intrafractional prostate motion during online image guided intensity-modulated radiotherapy for prostate cancer", 2011 Radiother Oncol (98:181-186)

45 B.M. Barney, R.J. Lee, D. Handrahan, et al., "Image-guided radiotherapy (IGRT) for prostate cancer comparing kV imaging of fiducial markers with cone beam computed tomography (CBCT)", 2011 Int J Radiat Oncol Biol Phys (80:301-305)

46 B.M. Barney, "Private communication", (2013).

47 D.J. Moseley, E.A. White, K.L. Wiltshire, et al., "Comparison of localization performance with implanted fiducial markers and cone-beam computed tomography for on-line image-guided radiotherapy of the prostate", 2007 Int J Radiat Oncol Biol Phys (67:942-953)

48 V. Narayana, P.L. Roberson, A.T. Pu, et al., "Impact of differences in ultrasound and computed tomography volumes on treatment planning of permanent prostate implants", 1997 Int J Radiat Oncol Biol Phys (37:1181-1185)

49 V. Narayana, P.L. Roberson, R.J. Winfield, P.W. McLaughlin, "Impact of ultrasound and computed tomography prostate volume registration on evaluation of permanent prostate implants", 1997 Int J Radiat Oncol Biol Phys (39:341-346)

50 S.C. Hoffelt, L.M. Marshall, M. Garzotto, et al., "A comparison of CT scan to transrectal ultrasoundmeasured prostate volume in untreated prostate cancer", 2003 Int J Radiat Oncol Biol Phys (57:29-32)

51 G. Delouya, J.F. Carrier, D. Beliveau-Nadeau, et al., "Migration of intraprostatic fiducial markers and its influence on the matching quality in external beam radiation therapy for prostate cancer", 2010 Radiother Oncol (96:43-47)

52 P.A. Kupelian, T.R. Willoughby, S.L. Meeks, et al., "Intraprostatic fiducials for localization of the prostate gland: monitoring intermarker distances during radiation therapy to test for marker stability", 2005 Int J Radiat Oncol Biol Phys (62:1291-1296)

53 J.A. Antolak, Rosen, II, C.H. Childress, et al., "Prostate target volume variations during a course of radiotherapy", 1998 International journal of radiation oncology, biology, physics (42:661-672)

54 K.E. Deurloo, R.J. Steenbakkers, L.J. Zijp, et al., "Quantification of shape variation of prostate and seminal vesicles during external beam radiotherapy", 2005 Int J Radiat Oncol Biol Phys (61:228-238)

55 K.M. Langen, D.T. Jones, "Organ motion and its management", 2001 International journal of radiation oncology, biology, physics (50:265-278)

56 J.C. Roeske, J.D. Forman, C.F. Mesina, et al., "Evaluation of changes in the size and location of the prostate, seminal vesicles, bladder, and rectum during a course of external beam radiation therapy", 1995 International journal of radiation oncology, biology, physics (33:1321-1329) 



\section{CHAPTER}

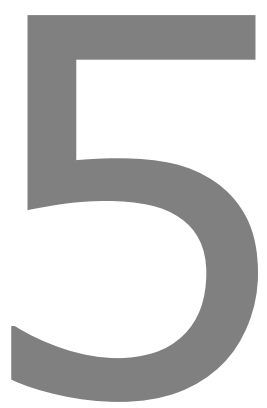

Active breathing control in combination with ultrasound imaging: a feasibility study of image guidance in stereotactic body radiation therapy of liver lesions

Esther Bloemen-van Gurp*, Skadi van der Meer*, Janet Hendry, Jeroen Buijsen, Peter Visser, Davide Fontanarosa, Martin Lachaine, Guido Lammering and Frank Verhaegen

*These authors contributed equally to this work. 


\section{ABSTRACT}

Purpose: Accurate tumor positioning in stereotactic body radiation therapy (SBRT) of liver lesions is often hampered by motion and setup errors. We combined 3-dimensional ultrasound imaging (3DUS) and active breathing control (ABC) as an image guidance tool.

Methods: We tested 3DUS image guidance in the SBRT treatment of liver lesions for 11 patients with 88 treatment fractions. In 5 patients, 3DUS imaging was combined with ABC. The uncertainties of US scanning and US image segmentation in liver lesions were determined with and without $A B C$.

Results: In free breathing, the intra-observer variations were $1.4 \mathrm{~mm}$ in left-right (L-R), 1.6 $\mathrm{mm}$ in superior-inferior (S-I), and $1.3 \mathrm{~mm}$ anterior-posterior (A-P). and the inter-observer variations were $1.6 \mathrm{~mm}(\mathrm{~L}-\mathrm{R}), 2.8 \mathrm{~mm}(\mathrm{~S}-\mathrm{I})$, and $1.2 \mathrm{~mm}(\mathrm{~A}-\mathrm{P})$. The combined uncertainty of US scanning and matching (inter- and intra-observer) was $4 \mathrm{~mm}$ (1 SD). The combined uncertainty when $A B C$ was used reduced by $1.7 \mathrm{~mm}$ in the S-I direction. For the L-R and A$P$ directions, no significant difference was observed.

Conclusions: 3DUS imaging for IGRT of liver lesions is feasible, although using anatomic surrogates in the close vicinity of the lesion may be needed. ABC-based breath-hold in midventilation during 3DUS imaging can reduce the uncertainty of US-based 3D table shift correction.

\section{Summary}

For image guidance, 3-dimensional ultrasound imaging in the stereotactic body radiation therapy of liver lesions is feasible. The use of surrogates in the close vicinity of lesions may be needed. The accuracy of 3-dimensional ultrasound image guidance is improved by using active breathing control; in free breathing the accuracy is $4 \mathrm{~mm}$, and when it is combined with active breathing control the accuracy is $2 \mathrm{~mm}$. 


\section{INTRODUCTION}

Stereotactic body radiation therapy (SBRT) of liver lesions requires tight margins for safe treatment with high radiation doses while limiting the normal liver dose. Accurate daily localization of the treatment target is highly important. Organ motion and patient setup are potential error sources. Currently, image guided radiation therapy (IGRT) is mostly based on x-ray imaging (e.g., electronic portal imaging [EPI] and cone beam [CB] computed tomography [CT]). For liver lesions, however, these techniques provide inadequate imaging contrast. EPI images of bony anatomy cannot deal with the liver motion related to breathing and stomach and bowel filling. Case et al. ${ }^{1}$ assessed the inter-fraction and intrafraction variability of the liver position, treated with KV CBCT-guided SBRT. They demonstrated that inter-fractional liver position relative to the vertebral bodies is a source of geometric uncertainty. To alleviate the poor x-ray imaging contrast in soft tissue, fiducial markers have been proposed as a surrogate. Marker-guided setup accuracy decreases with increasing distance between the fiducial markers and the tumor ${ }^{2}$. Furthermore, implanting fiducial markers is invasive and can result in tumor spread, liver inflammation, and embolization ${ }^{3}$. Although $\mathrm{kV} \mathrm{CBCT} \mathrm{may} \mathrm{allow} \mathrm{visualization} \mathrm{of} \mathrm{liver} \mathrm{boundaries,} \mathrm{the}$ lesion or nearby blood vessels are not clearly visible. Furthermore, CBCT image quality is limited because of breathing artifacts leading to motion aliasing. The time to acquire a CBCT image makes breathing control problematic. The recently reported respiratory correlated $\mathrm{CBCT}(4 \mathrm{D}-\mathrm{CBCT})^{1,4}$ may solve the motion-related artifacts but will not solve the poor soft-tissue contrast.

A clear need is identified for an alternative imaging technique to guide radiation therapy. A reduction of geometric uncertainties may lead to reduced normal tissue irradiation, safer dose escalation, and improved local control for patients with hepatic malignancies. Ultrasound (US) imaging may offer a solution. In radiation therapy this is a fairly new technique, although it has been used extensively for many years as 2-dimensional (2D) US imaging in diagnostic radiology because of the high soft tissue contrast of US imaging. The feasibility of US imaging was demonstrated ${ }^{5}$ for liver guidance, using the BAT device (Nomos, Cranberry Township, PA). This allows for superposition of anatomic contours derived from treatment planning CT onto real-time US images, for liver image guidance. The use of 2 different image modalities in an intermodality system, however, introduces additional uncertainty.

We investigated the use of an intramodality 3-dimensional (3D) US system as a novel IGRT application in liver treatment. The intramodality approach entails comparing US images acquired in the treatment room with a reference US image acquired at the time of CT simulation. The goal is to establish the accuracy of 3DUS imaging with and without active breathing control (ABC) for IGRT of liver lesions. 


\section{MATERIALS AND METHODS}

Eleven patients (Table 1 ) were included in this study (1 patient was not included in the study because of bad sonographic visibility). All patients gave written informed consent before entering this study, which was approved by the internal review board.

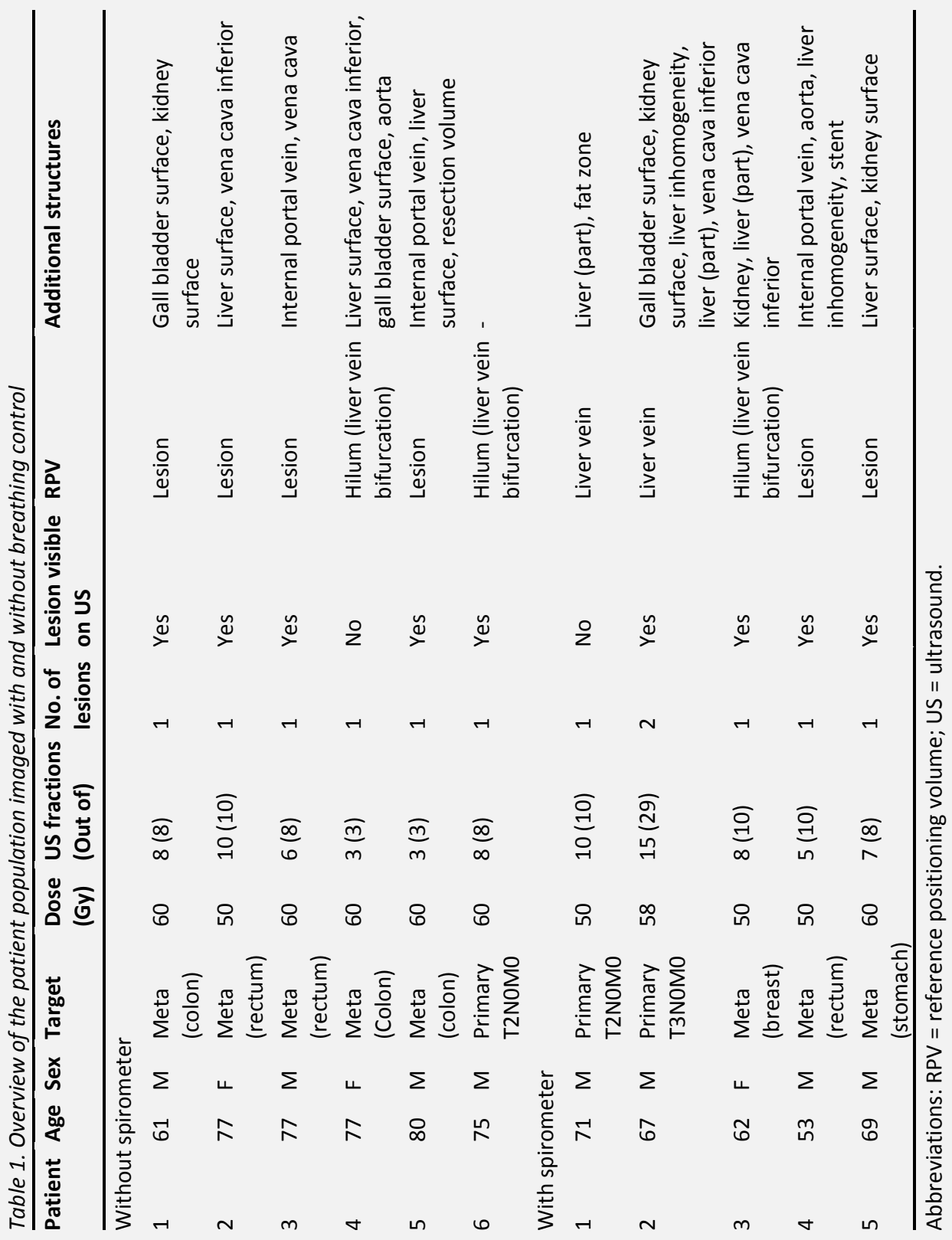




\section{DUS system}

The Clarity intramodality US imaging system (Elekta, Stockholm, Sweden) was used for this study in a total of 88 treatment fractions. The system comprises 2 interlinked US stations, based in the CT room (US-Sim) and in the treatment room (US-Guide). Each US station is equipped with a 2DUS probe $(3.3 \mathrm{MHz}$ ) designed for abdominal scanning and a ceilingmounted infrared stereovision camera. Four reflective markers are attached to the probe and are tracked in real time by the infrared camera to determine the position and orientation of each ultrasound frame. The 2DUS frames are then reconstructed in space to form a 3DUS voxel dataset. These 3DUS images are calibrated to the room coordinate system of the corresponding $\mathrm{CT}$ and treatment room to allow a direct comparison of the reference 3DUS images at simulation with those acquired in the treatment room (referred to as image segmentation). From this, the difference in daily position of the lesion is derived, resulting in an absolute shift to reposition the patient for treatment.

Our first experiences in liver scanning demonstrated that, with the current transabdominal probe design, it is difficult to maintain a direct line of visibility between the reflective markers and the optical tracking system during scanning. This is due to the position of the markers on the probe, the wide range of angles of the probe during scanning, and the range of positions of the probe on the skin under various orientations. To obtain optimal scanning and probe tracking conditions for IGRT in liver lesions, we redesigned, in collaboration with the manufacturer, the reflective marker array fitted onto the curved abdominal probe (Figure 1a). To ensure the accuracy of the prototype probe in relation to the room coordinates, a daily calibration check was performed before each treatment session. The uncertainty for each US station was within $1 \mathrm{~mm}$, resulting in a combined uncertainty of $2 \mathrm{~mm}$ for the whole quality assurance procedure.

\section{ABC system}

For breathing control we used the Spiro SDX system (DynR, France), designed for radiation therapy. The system relies on maintaining a certain breath-hold $(\mathrm{BH})$ volume to fix the position of the lesion absolutely in space. The spirometer is connected to the patient by use of a mouthpiece with a nose clip to prevent nasal air leakage (Figure 1b). The spirometry sensor measures the patient's breath flow (L/sec). A predefined $\mathrm{BH}$ level can be set, and video goggles guide the patients to this predefined level. We used $50 \%$ of the tidal volume for $\mathrm{BH}$ (midventilation, 50\% expiration), referring to the breathing phase used for treatment planning (50\% expiration based on respiratory-correlated CT imaging [RCCT]).

We verified that both spirometers used (in CT and treatment rooms) did not deviate by more than $0.01 \mathrm{~L}$ from a precisely known volume of $3 \mathrm{~L}$, well within the manufacturer's specified $2 \%$ uncertainty.

\section{US procedure}

We combined $A B C$ with breathing feedback to the patient, in combination with 3DUS imaging. Before the first session, the patient was trained to become comfortable with the spirometer and video goggles and to determine the individual tidal volume and midventilation $\mathrm{BH}$ level. The midventilation level, including a small tolerance $( \pm 0.05 \mathrm{~L})$ was dis- 
played as a bar on the video goggles, guiding patients to the correct $\mathrm{BH}$ position (Figure $1 \mathrm{c})$. The patient was then requested to repeat a $\mathrm{BH}$ at the same pulmonary volume level during $\mathrm{CT}$ and radiation therapy sessions.

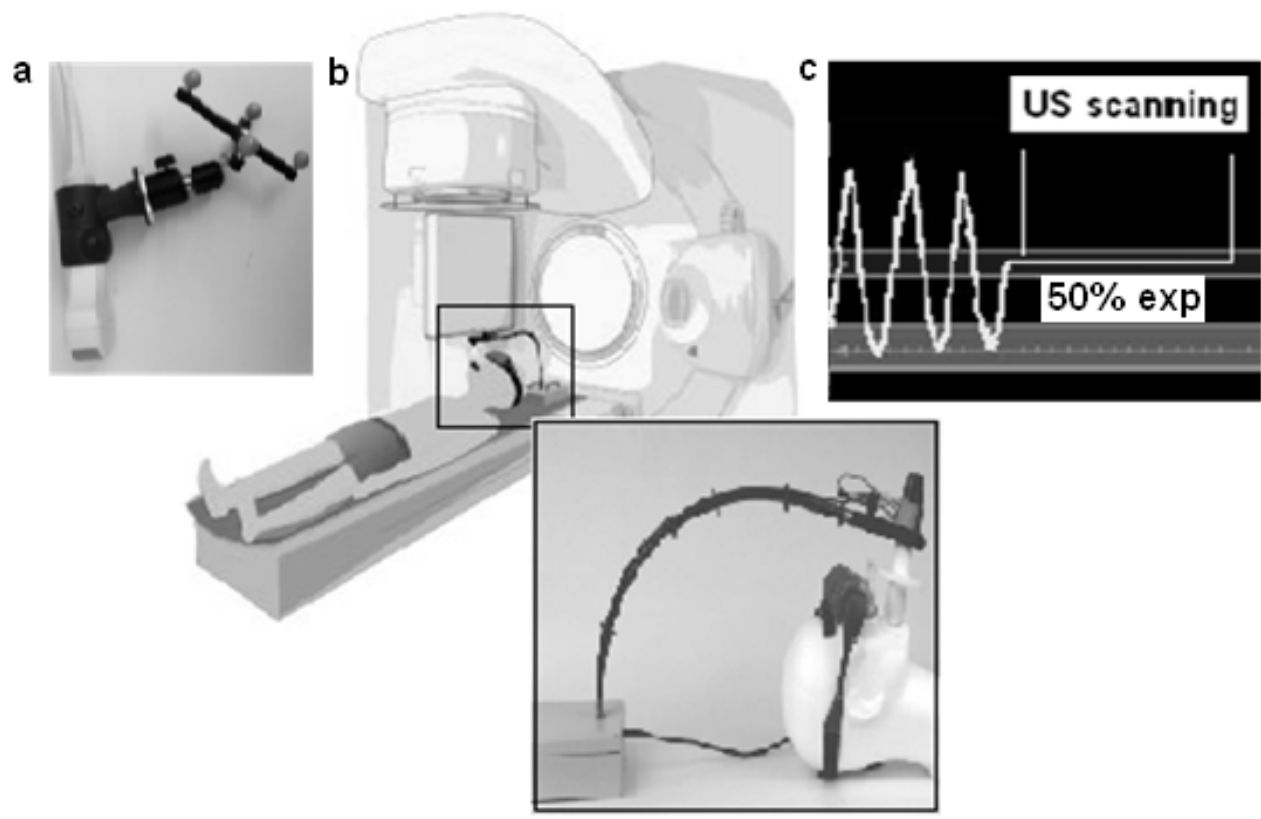

Figure 1: (a) Prototype of US probe designed for optimal scanning of liver and surrounding structures. (b) SDX spirometer system. (c) display of the breathing signal and sequential BH in 50\% expiration, used for US scanning.

The standard procedure for CT simulation was followed for treatment planning. Patients were positioned supine with the arms above the head. A 4D helical RCCT scan was made with the patient in the treatment position (SOMATOM Sensation Open, Syngo CT 2006A, Siemens, Germany, $3 \mathrm{~mm}$ slice thickness). Small markers (BBs) were placed on the laser crossings on the patient's skin to indicate the isocenter of the CT. CT contrast medium was injected by an intravenous line directly before scanning. During CT scanning, the patient's respiration signal was recorded by use of a thorax band with a pressure sensor (Anzai, Germany) to allow respiration phase sorting of the images. The $50 \%$ expiration phase was used for treatment planning. Directly after the RCCT scan, a reference transabdominal US scan in midventilation $\mathrm{BH}$ was made. For scanning, the probe was placed between 2 ribs or directly under the lowest rib, depending on the position of the liver lesion. The probe was swept over the whole area of interest (lesion and surrounding structures), acquiring up to 250 image frames. This $2 \mathrm{D}$ image dataset was reconstructed into an axial 3D voxel dataset. After scanning, the location of the probe was marked on the patient's skin to enable capturing approximately the same US volume in subsequent sessions.

The US image was automatically registered to the CT image, inasmuch as the 3D voxel dataset is calibrated to the room coordinate system of the CT scanner. The CT/US registration was verified by a trained radiation technologist by comparing the position of the 
lesion(s), liver veins and the liver surface on the CT and US images. CT contours were used as an additional tool to evaluate the fusion. In case of a mismatch between the 2 scans (caused, for example, by patient motion between acquisitions), the US scan was manually shifted to the coordinates of the CT scan to obtain an optimal overall match. Next, the reference positioning volume (RPV), the reference structure for imaging and segmentation in the treatment room, was contoured on the US scan. Additional structures of interest (surrogates) were also contoured, such as the hepatic and internal portal veins, the vena cava inferior, and the surfaces of the liver, kidney, and gall bladder, depending on their visibility. The RPV and surrogates used in our patient group are described in Table 1 . We were not always able to use the automatic contouring tools available in the Clarity software because of the lack of specific tools for liver (the automatic contouring software is currently optimized for imaging of the prostate, breast, bladder, gynecologic organs). Manual contouring was performed when automatic contouring was not possible. To complete the preparation process, the isocenter and beams were imported from the treatment planning system into Clarity. A physician approved the RPV for image guidance.

For treatment, our standard online EPI correction procedure was followed. Directly after the treatment, additional EPIs were performed, and the residual shift as a result of patient movement during treatment was determined. This was followed by US liver scan acquisition, using the same probe position and scanning technique as was used during treatment preparation. Four sequential US scans were acquired during each treatment session: 2 US scans in free breathing (FB) and 2 scans in BH. US scans with and without $A B C$ were performed to investigate the accuracy of 3DUS IGRT of liver lesions in FB and $\mathrm{BH}$, respectively, to establish the optimal IGRT workflow. Repeated scanning was performed to investigate the total uncertainty as a result of repeated scanning in combination with image segmentation (referred to as repeated scan and match uncertainty). To minimize the time between the 4 US scans, image segmentation was performed retrospectively on the workstation. The review software, designed as a tool to evaluate daily imaging and US segmentation, was also used for training purposes. It is possible to simulate the whole image segmentation procedure in a manner similar to the procedure used on the US guide. The advantage of using this tool is that image segmentation can be performed several times by different observers. Each observer is blinded to the results of the previous observer. These shifts were documented for future analysis. We were not able to use the automatic Adapt function, which adjusts the reference contour to the grey-level boundaries of the current contour because it was not designed for liver greyscale values and is thus not included for liver lesion segmentation. Furthermore, no corrective rotations were calculated by the software for this analysis.

\section{Accuracy of 3DUS image guidance in liver lesions}

The accuracy of 3DUS image guidance in liver lesions was determined for both the FB and $\mathrm{BH}$ scans and was based on the mean directional difference within or between observers. The directional difference was defined between the RPV and the actual target position and was determined by manually aligning the RPV to the underlying anatomy of the actual US image. The resulting 3D table shift was independently assessed by 3 observers. In case of (image) deformation, it was impossible to correctly align both RPV and surrogates 
accurately to the predefined structures. In these cases, predefined surrogates at larger distances from the lesion were given less weight in determining the correct alignment compared with the RPV and surrogates close to the lesion.

The inter-observer variation was defined as the mean standard deviation (SD) of the directional difference between 3 observers. It was determined for $\mathrm{BH}$ and $\mathrm{FB}$. The intra observer variation was calculated as the mean SD of the mean directional difference within observers and was determined by selecting randomly for each patient 4 US scans (2 $\mathrm{BH}$ and $2 \mathrm{FB}$ scans). The directional shift was determined 5 times in a random order by 3 observers. The variation was defined as the mean SD of the directional shift of the 5 repeated segmentations, averaged over the 3 observers.

The repeated scan variation combined the uncertainty resulting from both repeated scanning and repeated image segmentation. This was determined for $\mathrm{BH}$ and $\mathrm{FB}$ and was defined as the mean SD of the directional difference between 2 sequential US scans acquired within a treatment fraction by the same scanner and segmented by 3 observers. The results were averaged over all treatment fractions and over the 3 observers. In all, 184 US scans were used (5 patients, 46 treatment fractions).

\section{RESULTS}

In 6 of 11 patients, a manual fusion was deemed necessary because of an offset between the CT and US structures. The median offset was $6.4 \mathrm{~mm}$. Eleven patients with 88 treatment fractions were included in the study. In 6 of them, the lesion was used as a RPV. In the other 5 patients, the lesion could not be used for image segmentation because it was not completely displayed on the US image, owing to its size or shadowing of the ribs, or the structure itself did not have sufficient contrast for accurate delineation. In 6 of the 11 patients, for a total of 42 treatment fractions, we determined the accuracy of 3DUS for image segmentation without the use of $A B C$. In 5 patients, for a total of 46 treatment fractions, we determined the accuracy of 3DUS with $A B C$.

The mean tidal volume of the 5 patients was $0.32 \pm 0.19 \mathrm{~L}$. The average tolerance around the midventilation level was $0.05 \pm 0.02 \mathrm{~L}$. All patients were able to hold their breath for at least 10 to 15 seconds, which was sufficient for US scanning. An overview of the patient population is given in Table 1.

\section{Accuracy of 3DUS image guidance in liver lesions}

The mean intra-observer variation of the 3 observers is illustrated in Figure 2a. The FB intra-observer variations were $1.4 \mathrm{~mm}$ in L-R $(0.7-1.7 \mathrm{~mm}), 1.6 \mathrm{~mm}$ in S-I $(1.0-2.2 \mathrm{~mm})$, and $1.3 \mathrm{~mm}$ in A-P $(0.9-1.6 \mathrm{~mm})$ directions. The intra-observer variations for $\mathrm{BH}$ were 1.2 $\mathrm{mm}$ in L-R $(0.6-1.8 \mathrm{~mm}), 1.4 \mathrm{~mm}$ in S-I $(0.8-1.8 \mathrm{~mm})$, and $1.3 \mathrm{~mm}$ in A-P $(0.8-1.5 \mathrm{~mm})$ directions. No significant difference in intra-observer variation was observed between $\mathrm{FB}$ and $\mathrm{BH}$. 


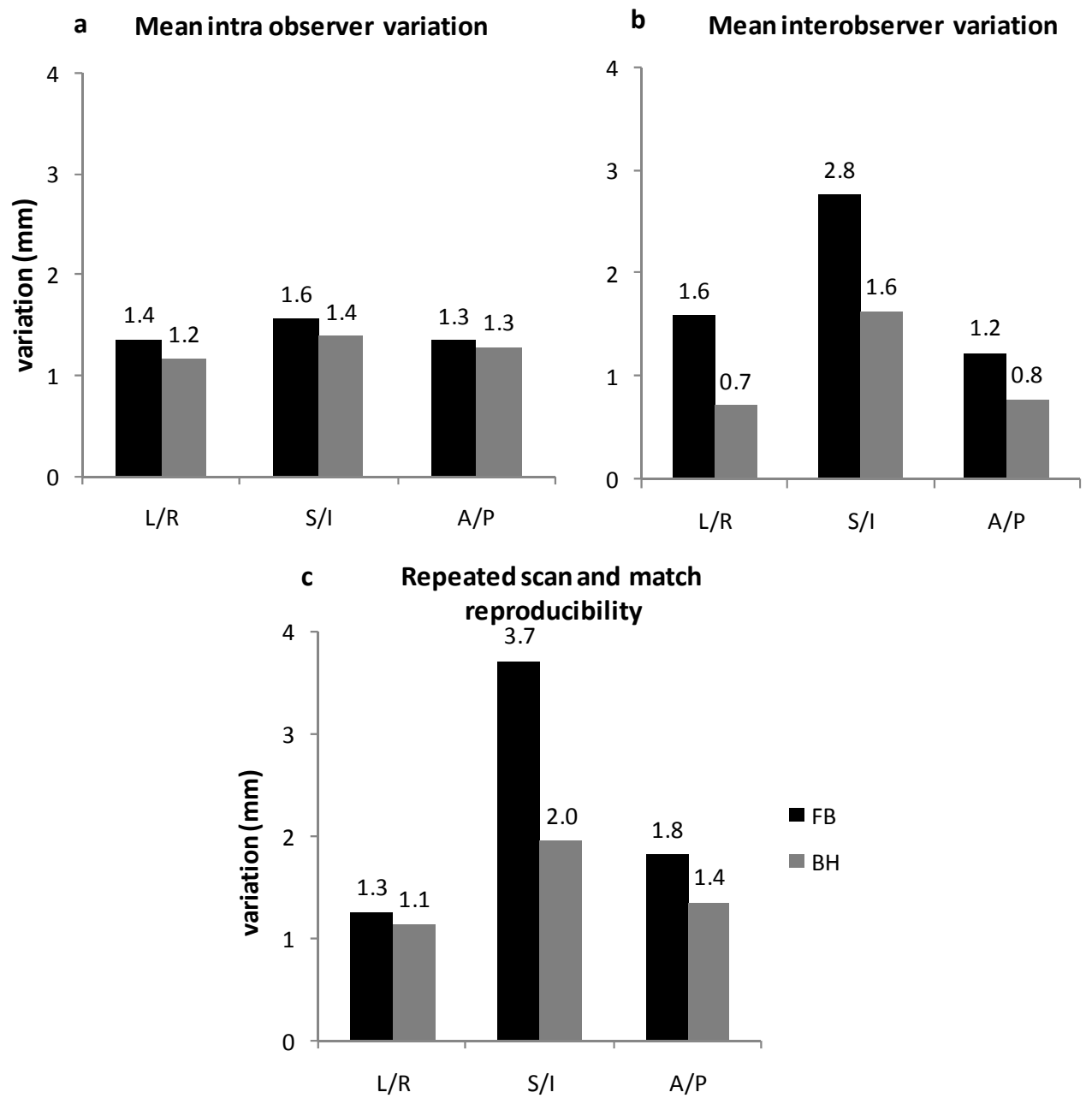

Figure 2: Intra-observer variability (a) and inter-observer variability (b) determined for free breathing and breath-hold ultrasound scanning. (c) Repeated scan and match reproducibility.

The inter-observer variation is illustrated in Figure $2 \mathrm{~b}$. For $\mathrm{FB}$, the variations were $1.6 \mathrm{~mm}$ in L-R $(0.3-2.7 \mathrm{~mm}), 2.8 \mathrm{~mm}$ in S-I $(0.8-3.8 \mathrm{~mm})$, and $1.2 \mathrm{~mm}$ in A-P $(0.6-2.1 \mathrm{~mm})$ directions. For $B H$, the variations were $0.7 \mathrm{~mm}$ in $\mathrm{L}-\mathrm{R}(0.1-1.6 \mathrm{~mm}), 1.6 \mathrm{~mm}$ in S-I $(0.6-2.5 \mathrm{~mm})$, and $0.8 \mathrm{~mm}$ in A-P $(0.2-1.9 \mathrm{~mm})$ directions. When the $\mathrm{FB}$ and $\mathrm{BH}$ results were compared, the uncertainty reduced by $1.2 \mathrm{~mm}$ in the S-I direction using BH scans. In the A-P direction, no significant difference was observed, and in the L-R direction, a reduction of $0.9 \mathrm{~mm}$ was found.

The combined uncertainty of repeated scan uncertainty and inter- and intra-observer variation is illustrated in Figure 2c. For FB, the variations were $1.3 \mathrm{~mm}$ in $\mathrm{L}-\mathrm{R}(0.5-4.1 \mathrm{~mm})$, $3.7 \mathrm{~mm}$ in $\mathrm{SI}(1.5-7.1 \mathrm{~mm})$, and $1.8 \mathrm{~mm}$ in A-P $(1.0-2.9 \mathrm{~mm})$ directions. For $\mathrm{BH}$, the uncertainties were $1.1 \mathrm{~mm}$ in L-R $(0.3-2.8 \mathrm{~mm}), 2.0 \mathrm{~mm}$ in S-I $(1.2-2.9 \mathrm{~mm})$, and $1.4 \mathrm{~mm}$ in A-P 
(0.6-1.9 mm) directions. When FB and $\mathrm{BH}$ were compared, the mean uncertainty reduced by $1.7 \mathrm{~mm}$ in the S-I direction. For the L-R and A-P directions, no difference was observed.

\section{DISCUSSION}

This study demonstrates that 3DUS imaging for image guidance in SBRT of liver lesions is feasible. The importance of US IGRT (BAT device) for upper abdominal lesions has previously been shown. Boda-Heggeman et al..$^{5}$ demonstrated, using US image guidance after EPI image segmentation, a significant difference between the intended and actual positions of the liver lesion. Fuss et al. ${ }^{6}$ validated their residual shifts measured with a BAT system in 15 patients with abdominal malignancies, by repeated CT scanning. The CT analysis demonstrated that 14 of the suggested BAT shifts reduced the initial setup error by $15-95 \%$ compared with room laser alignment.

The accuracy of 3DUS imaging for image guidance in SBRT of liver lesions is often hampered by breathing motion. ABC-based US imaging leads to reduced blurring and reduced artifacts in the $3 D$ generated US data. As a result, reduced intra- and interobserver variability in 3DUS-based IGRT was observed. Using $\mathrm{BH}$ during imaging reduced especially the uncertainty in the S-I direction. The effect of breathing on image quality depends on the patient's breathing pattern (frequency and amplitude). In addition, breathing causes the liver to deform ${ }^{7}$, and this changes the relationship between the lesion and surrogates in the liver (Figure 3). Using an absolute $\mathrm{BH}$ volume during the sequential sessions optimizes image quality and results in a similar position of the lesion during image guidance compared with the treatment planning situation.

The midventilation level was $0.32 \pm 0.07 \mathrm{~L}$, meaning that the average tolerance window for $\mathrm{BH}$ was $\sim 45 \%$ of the total tidal volume. Our patients (mean age, 71 years) were not able to hold their breath within the bar when its size was reduced. This may influence the repeated scan and match uncertainty. For 2 sequential US scans, the patients had 2 sequential BHs. The effect of the breathing uncertainty is expected to be small.

Our US scanning technique of superficial lesions between the ribs resulted in a small field of view. For deeper seated lesions, we preferably scanned abdominally under the lowest rib. Using additional guidance structures in the vicinity of the liver lesion was indispensable for accurate IGRT in liver lesions. In 2 of 11 patients, the lesion was not visible on the US image. In 3 patients, the lesion was visible; however, it was too large to fit within the US field of view because of its location directly under the ribs, or it was not sufficiently echogenic for accurate image segmentation. In these cases, liver veins close to the lesion, preferably with a clearly visible bifurcation (e.g., hilum), were used as primary reference structure. This approach has been previously described ${ }^{6}$. In case of deformations, surrogates farther away from the lesion were given less weight than were surrogates close to the lesion when alignments were performed. Structures and regions with motion artifacts were excluded from consideration. As with the currently available Clarity US prostate solution, proper training will avoid the need for a diagnostic radiologist to be present for liver scanning. 

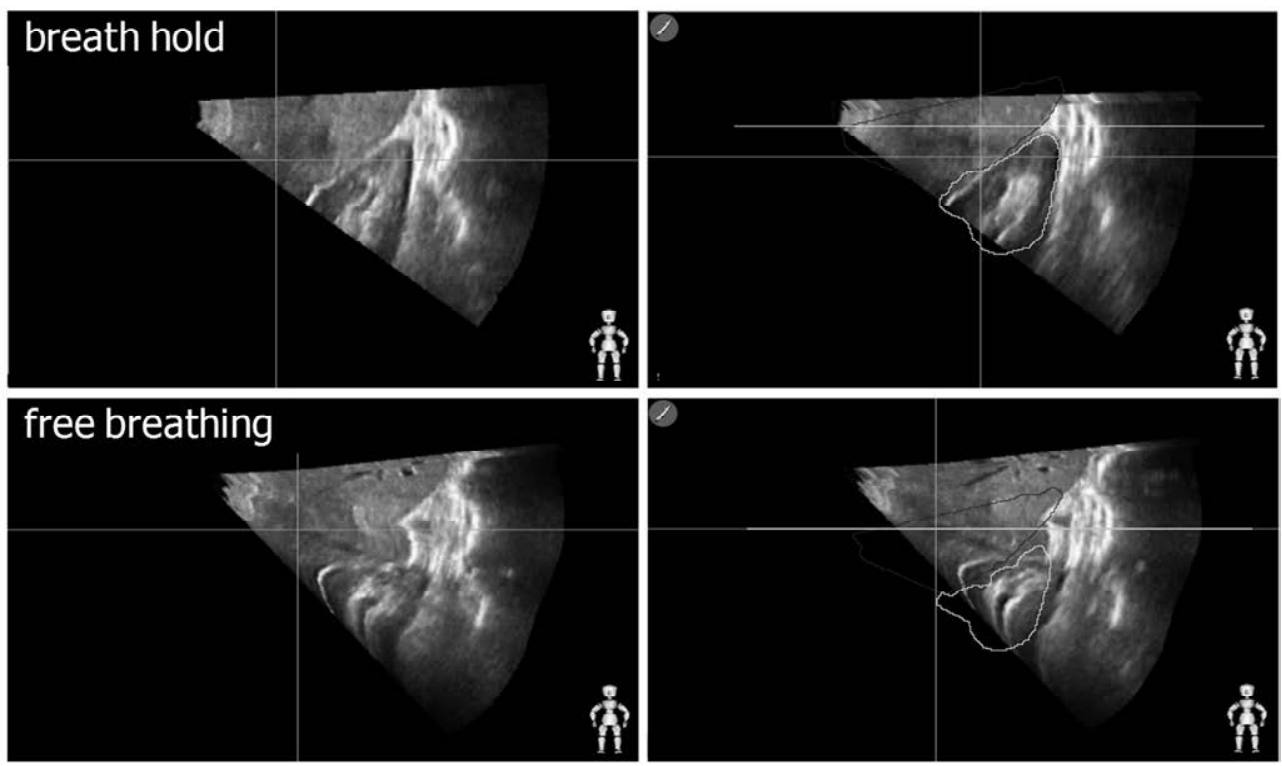

Figure 3: Ultrasound image of part of the liver (contoured in black) and the right kidney (contoured in white) acquired in breath-hold and free breathing, respectively. Deformation due to motion artifacts is clearly visible in the free breathing images compared with the breathhold images. They increase the uncertainty of image segmentation.

Clarity uses an intramodality workflow comparing US reference with current US images. Uncertainties as a result of speed of sound aberrations ${ }^{8,9}$ are thus limited. One should, however, be aware during US and CT fusion that uncertainties as a result of speed of sound errors play a role for deep-seated structures and that the fusion at this depth will have reduced accuracy. In our patient group, the difference between US and CT for deepseated structures was on average $3.5 \mathrm{~mm}$. When manual CT US fusion has to be performed, one should be aware of this effect. Manual fusion should be based on superficial structures, accepting differences at depth.

Replacing image guidance based on bony landmarks by direct visualization of liver lesions may allow reducing setup margins and consequently reducing the dose to healthy tissues. This needs to be evaluated in dosimetric evolution studies of 3DUS IGRT, possibly in comparisons with other marker-based or nonmarker-based 3D guidance systems.

This, however, will be limited by a lack of breathing control during treatment. The long-term aim of this work is to implement breath-holding control during treatment (gated irradiation) and to reduce treatment margins as a result of eliminating the tumor motion effect on the PTV margins. This may then enable dose escalation and hypofractionation studies. 


\section{CONCLUSION}

3DUS imaging for image guidance in SBRT of liver lesions is feasible, although surrogates in the close vicinity of the lesion may need to be used. ABC-based breath-hold in midventilation during 3DUS imaging leads to reduced intra- and inter-observer variability in 3DUSbased 3D table shift correction.

\section{REFERENCES}

1 R.B. Case, J.J. Sonke, D.J. Moseley, et al., "Inter- and intrafraction variability in liver position in non-breathhold stereotactic body radiotherapy", 2009 Int J Radiat Oncol Biol Phys (75:302-308)

2 Y. Seppenwoolde, W. Wunderink, S.R. Wunderink-van Veen, et al., "Treatment precision of image-guided liver SBRT using implanted fiducial markers depends on marker-tumor distance", 2011 Phys Med Biol (56:5445-5468)

3 H. Hennessey, D. Valenti, T. Cabrera, et al., "Cardiac embolization of an implanted fiducial marker for hepatic stereotactic body radiotherapy: a case report", $2009 \mathrm{~J}$ Med Case Reports (3:140)

4 J.J. Sonke, L. Zijp, P. Remeijer, M. van Herk, "Respiratory correlated cone beam CT", 2005 Med Phys (32:1176-1186)

5 J. Boda-Heggemann, C. Walter, S. Mai, et al., "Frameless stereotactic radiosurgery of a solitary liver metastasis using active breathing control and stereotactic ultrasound", 2006 Strahlenther Onkol (182:216221)

6 M. Fuss, B.J. Salter, S.X. Cavanaugh, et al., "Daily ultrasound-based image-guided targeting for radiotherapy of upper abdominal malignancies", 2004 Int J Radiat Oncol Biol Phys (59:1245-1256)

7 M. Velec, J.L. Moseley, C.L. Eccles, et al., "Effect of breathing motion on radiotherapy dose accumulation in the abdomen using deformable registration", 2010 Int J Radiat Oncol Biol Phys (80:265-272)

8 D. Fontanarosa, S. van der Meer, E. Harris, F. Verhaegen, "A CT based correction method for speed of sound aberration for ultrasound based image guided radiotherapy", 2011 Med Phys (38:2665-2673)

9 B.J. Salter, B. Wang, M.W. Szegedi, et al., "Evaluation of alignment error due to a speed artifact in stereotactic ultrasound image guidance", 2008 Phys Med Biol (53:N437-445) 


\section{APPENDIX}

(Not part of the publication Int J Radiat Oncol Biol Phys 85(4):1096-1102, 2013)

\section{Methods}

Comparison in patient positioning: US versus EPI shift

In addition to the feasibility study of ultrasound (US) image guided radiotherapy (IGRT) in stereotactic body radiation therapy (SBRT) of liver lesions, a retrospective comparison between image guidance based on 3DUS (Clarity system, Elekta, Stockholm, Sweden) and megavolt (MV) electronic portal imaging (EPI) images (Optivue AN9, Siemens, Germany) was performed. Only for eight out of the eleven original patients there where US and EPI correction shifts available, resulting in 63 treatment fractions in total [approved by the Internal Review Board (IRB)]. In six patients the lesion was visible on the US image, but only in four of them the lesion was used as a reference positioning volume (RPV). In the other two patients the lesion was visible but could not be used for image segmentation because it was not completely displayed on the US image due to its size or shadowing of the ribs, or the contours of the lesion were not bright enough for accurate image segmentation. When the lesion was not visible, or could not be used, nearby liver veins where used as surrogate for the US localization.

In six of the eight patients (38 treatment fractions), we compared 3DUS without breathing control to EPI. In the other two patients ( 25 treatment fractions) we compared 3DUS with breathing control to EPI. An overview of the patient population is given in Table A (Condensed version of Table 1, Chapter 5).

Table A: patient overview (Condensed version of Table 1, Chapter 5)

\begin{tabular}{lllll}
\hline Patient & Dose (Gy) & \# Fractions & $\begin{array}{l}\text { Breathing control } \\
\text { during US }\end{array}$ & RPV for US positioning \\
\hline 1 & 60 & 8 & No & Lesion \\
2 & 50 & 10 & No & Lesion \\
3 & 60 & 8 (only 6 with US) & No & Lesion \\
4 & 60 & 3 & No & Hilum (Liver vein bifurcation) \\
5 & 60 & 3 & No & Lesion \\
6 & 60 & 8 & No & Hilum (Liver vein bifurcation) \\
7 & 50 & 10 & Yes & Liver vein \\
8 & 58 & 29 (only 15 with US) & Yes & Liver vein \\
\hline
\end{tabular}

The US image acquisition is fully described in the main part of this chapter. The US correction shift from that study are averaged per fraction and in this study compared to EPI shifts (Theraview-NT 2.4, Cablon Medical, Leusden, the Netherlands). EPI image guidance was based on the position of bone structures (vertebrae and ribs) on the digitally reconstructed radiograph (DRR), and compared to the position of these structures on the 
orthogonal EPIs. The DRR is a digital projection of the CT data representing an anteriorposterior and lateral view of the scanned area, in this case reconstructed from the $50 \%$ expiration CT scan. For treatment, our standard online EPI correction procedure was followed. Directly after the treatment, additional EPIs were performed, and the residual shift as a result of patient movement during treatment was determined. This was followed by US liver scan acquisition, using the same probe position and scanning technique as was used during treatment preparation ${ }^{1}$.

Like in chapter 4, the difference between MV EPI and US localization is depicted in a correlation plot, a Bland-Altman plot, ${ }^{2}$ and a histogram. The correlation plot contains a regression line and the coefficient of determination $\left(R^{2}\right)$. In the Bland-Altman plot, the limits of agreement (LoA) (bias $\pm 1.96 * S D$ ) cover $95 \%$ of the measurements and are a measure whether the two methods are interchangeable. The confidence intervals $(\mathrm{Cl} ; \pm$ $1.96 *$ error, error ${ }_{\text {mean }}=\mathrm{V}\left(\mathrm{s}^{2} / \mathrm{n}\right)$, $\left.^{\text {error }} \mathrm{SD}=\mathrm{V}\left(3 \mathrm{~s}^{2} / \mathrm{n}\right)\right)$ describe the uncertainty in the calculated values. The $95 \%$ LoA depends on the assumption that the differences are from an approximately normal distribution; frequency histograms are plotted to check if this assumption is valid. If the LoA are larger than a clinically relevant difference one would accept that changing the method to treat the patient will cause a relevant difference.

\section{Dose calculation and evaluation}

To evaluate the dose effects of US IGRT for SBRT of liver lesions we assumed that the US shifts are representative of the actual target shifts. We therefore shifted the contours of the clinical target volume (CTV) and some OAR (liver and kidneys) with the US shifts. To simulate the consequences of an EPI or US shift, the original treatment beams are shifted with either the US or EPI shifts and the dose deposition for these shifted plans on the original plannings $\mathrm{CT}$ is recalculated.

The OAR spinal cord, which is not likely to move together with the tracked RPV, is only moved with the EPI shift that represents the bone shift. Dose volume histograms (DVH) are calculated for each fraction with US shifts available together with the total dose that would have been given if these fractions represent the whole treatment. The $D_{95}$ and $V_{90}$ of the CTV and some OAR constraints will be reported for each patient.

\section{Results}

\section{Patient positioning}

The differences between MV EPI and US prostate localization range in the left-right (LR) direction from -11.8 to $7.4 \mathrm{~mm}$, in the superior-inferior (SI) direction from -17.7 to 19.9 $\mathrm{mm}$ and in the anterior-posterior (AP) direction from -12.2 to $14.5 \mathrm{~mm}$. For the $\mathrm{LR}, \mathrm{SI}$, and AP direction separately, respectively, $71 \%, 48 \%$, and $71 \%$ of the measured differences are below $5 \mathrm{~mm}$. Nevertheless, in $73 \%$ at least one of the directions has a difference above 5 $\mathrm{mm}$ (for $10 \mathrm{~mm}$, this is $35 \%$ ). The average differences $\pm \mathrm{SD}$ are: $-2.0 \pm 4.1 \mathrm{~mm}$ (LR; Cl: $-3.0-$ $-1.0 \mathrm{~mm}$ ), $4.5 \pm 6.7 \mathrm{~mm}$ (SI; $\mathrm{Cl}: 2.8-6.1 \mathrm{~mm}$ ), and $1.2 \pm 5.3 \mathrm{~mm}(\mathrm{AP} ; \mathrm{Cl}:-0.1-2.5 \mathrm{~mm})$. This leads to LoA of -10.0-5.9 mm [LR, Figure A(b); $C_{\text {LowerLimit }}$-11.7--8.2 mm, Cl UpperLimit $: 4.2-7.7$ 
$\mathrm{mm}$ ], -8.7-17.6 mm [SI, Figure A(e); $\mathrm{Cl}_{\text {LowerLimit: }}$-11.5--5.8 mm, $\mathrm{Cl}_{\text {UpperLimit: }}:$ 14.8-20.5 mm], and -9.1-11.5 mm [AP, Figure $A(h)$; $C_{\text {LowerLimit }}$ :-11.4--6.9 mm, $\mathrm{Cl}_{\text {UpperLimit }}:$ 9.3-13.8 mm]. For the all three directions, there is no correlation between the two localization methods [LR: $R^{2}=0.14$, Figure $A(a) ; S I: R^{2}=0.01$, Figure $A(d) ; A P: R^{2}=0.00$, Figure $\left.A(g)\right]$. The frequency histograms of the differences follow approximately a normal distribution.

Because part of the patients had active breathing control ( $A B C$ ) during the US acquisition, the results are also shown for the two separate groups. The results for the US acquired during free breathing are shown in figure $B$, and in figure $C$ the results are shown while applying breathing control. A total overview of the results is given in Table B.

Table B: Overview of the results for the differences between MV EPI and US prostate localization.

\begin{tabular}{|c|c|c|c|c|}
\hline & & $\begin{array}{c}\text { No Breathing } \\
\text { control during US }\end{array}$ & $\begin{array}{l}\text { Breathing control } \\
\text { during US }\end{array}$ & All measurements \\
\hline Number of patients & & 6 & 2 & 8 \\
\hline Number of measurements & $\mathrm{n}$ & 38 & 25 & 63 \\
\hline \multirow[t]{3}{*}{ Mean diff. \pm SD } & $\Delta \mathrm{LR}$ & $-1.3 \pm 3.7 \mathrm{~mm}$ & $-3.1 \pm 4.4 \mathrm{~mm}$ & $-2.0 \pm 4.1 \mathrm{~mm}$ \\
\hline & $\Delta \mathrm{SI}$ & $2.0 \pm 6.9 \mathrm{~mm}$ & $8.2 \pm 4.3 \mathrm{~mm}$ & $4.5 \pm 6.7 \mathrm{~mm}$ \\
\hline & $\triangle \mathrm{AP}$ & $3.4 \pm 4.8 \mathrm{~mm}$ & $-2.1 \pm 4.1 \mathrm{~mm}$ & $1.2 \pm 5.3 \mathrm{~mm}$ \\
\hline \multirow[t]{3}{*}{ Range } & $\Delta \mathrm{LR}$ & $-11.8-6.0 \mathrm{~mm}$ & $-9.3-7.4 \mathrm{~mm}$ & $-11.8-7.4 \mathrm{~mm}$ \\
\hline & $\Delta \mathrm{SI}$ & $-17.7-19.9 \mathrm{~mm}$ & $-0.9-14.4 \mathrm{~mm}$ & $-17.7-19.9 \mathrm{~mm}$ \\
\hline & $\triangle \mathrm{AP}$ & $-5.6-14.5 \mathrm{~mm}$ & $-12.2-5.2 \mathrm{~mm}$ & $-12.2-14.5 \mathrm{~mm}$ \\
\hline \multirow[t]{3}{*}{$R^{2}$} & LR & 0.06 & 0.17 & 0.14 \\
\hline & SI & 0.18 & 0.04 & 0.01 \\
\hline & AP & 0.01 & 0.02 & 0.00 \\
\hline \multirow[t]{3}{*}{$<5 \mathrm{~mm}$} & $\Delta \mathrm{LR}$ & $84 \%$ & $52 \%$ & $71 \%$ \\
\hline & $\Delta \mathrm{SI}$ & $63 \%$ & $24 \%$ & $48 \%$ \\
\hline & $\triangle \mathrm{AP}$ & $66 \%$ & $80 \%$ & $71 \%$ \\
\hline$\Delta \mathrm{LR}$ or $\Delta \mathrm{SI}$ or $\Delta \mathrm{AP}>5 \mathrm{~mm}$ & & $61 \%$ & $92 \%$ & $73 \%$ \\
\hline$\Delta \mathrm{LR}$ or $\Delta \mathrm{SI}$ or $\Delta \mathrm{AP}>10 \mathrm{~mm}$ & & $32 \%$ & $40 \%$ & $35 \%$ \\
\hline
\end{tabular}



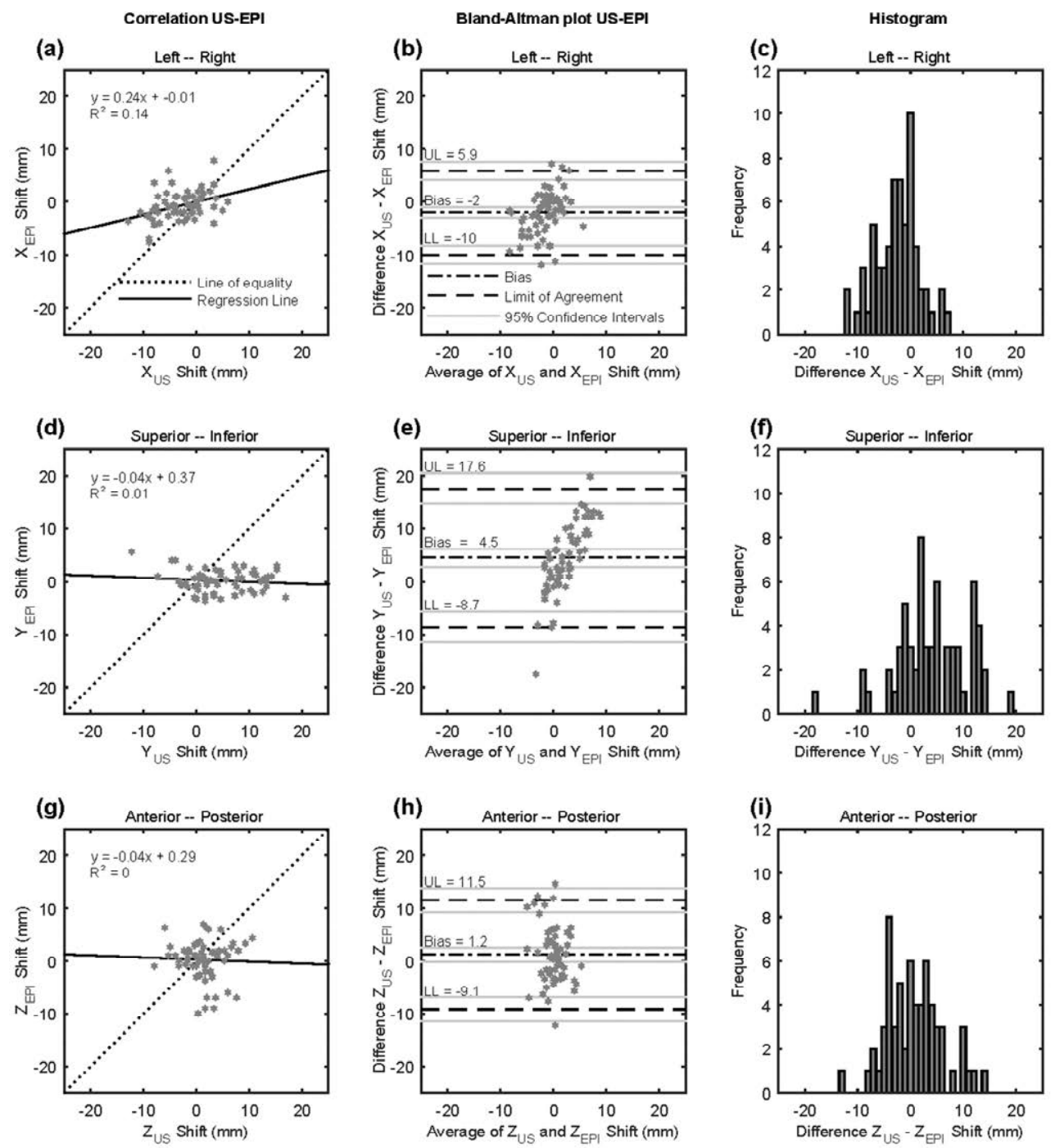

Figure A: Shifts measured with US localization vs MV portal image-based bone shifts shown in correlation plots $(a, d, g)$, Bland-Altman analysis $(b, e, h)$, and their accompanying histograms $(c, f, i)$. (Abbreviations: UL, upper limit; LL, lower limit). 


\section{No Breathing Control during US acquisition}

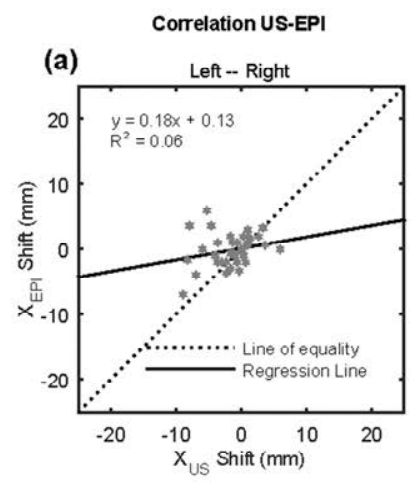

(b)

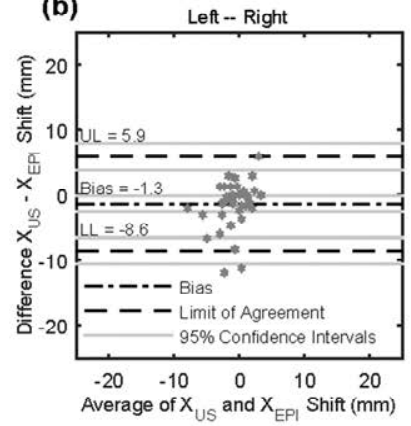

(e)

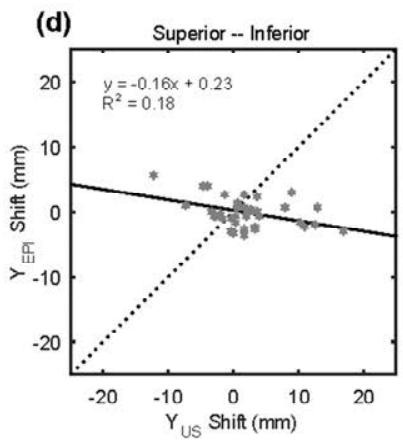

(g)
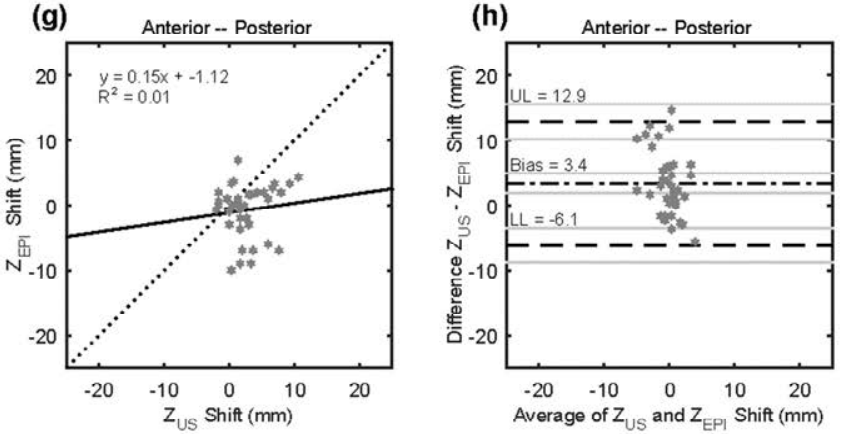

Bland-Altman plot US-EPI
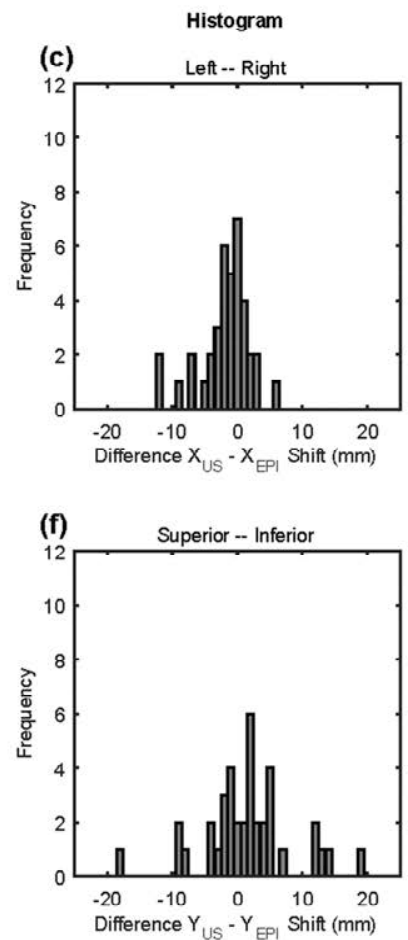

(i) Anterior -- Posterior

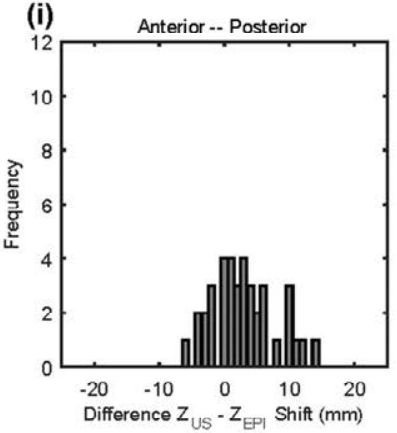

Figure B: Shifts measured with US localization vs MV portal image-based bone shifts shown in correlation plots $(a, d, g)$, Bland-Altman analysis $(b, e, h)$, and their accompanying histograms $(c, f, i)$. (Abbreviations: UL, upper limit; $L L$, lower limit). 


\section{Breathing Control during US acquisition}
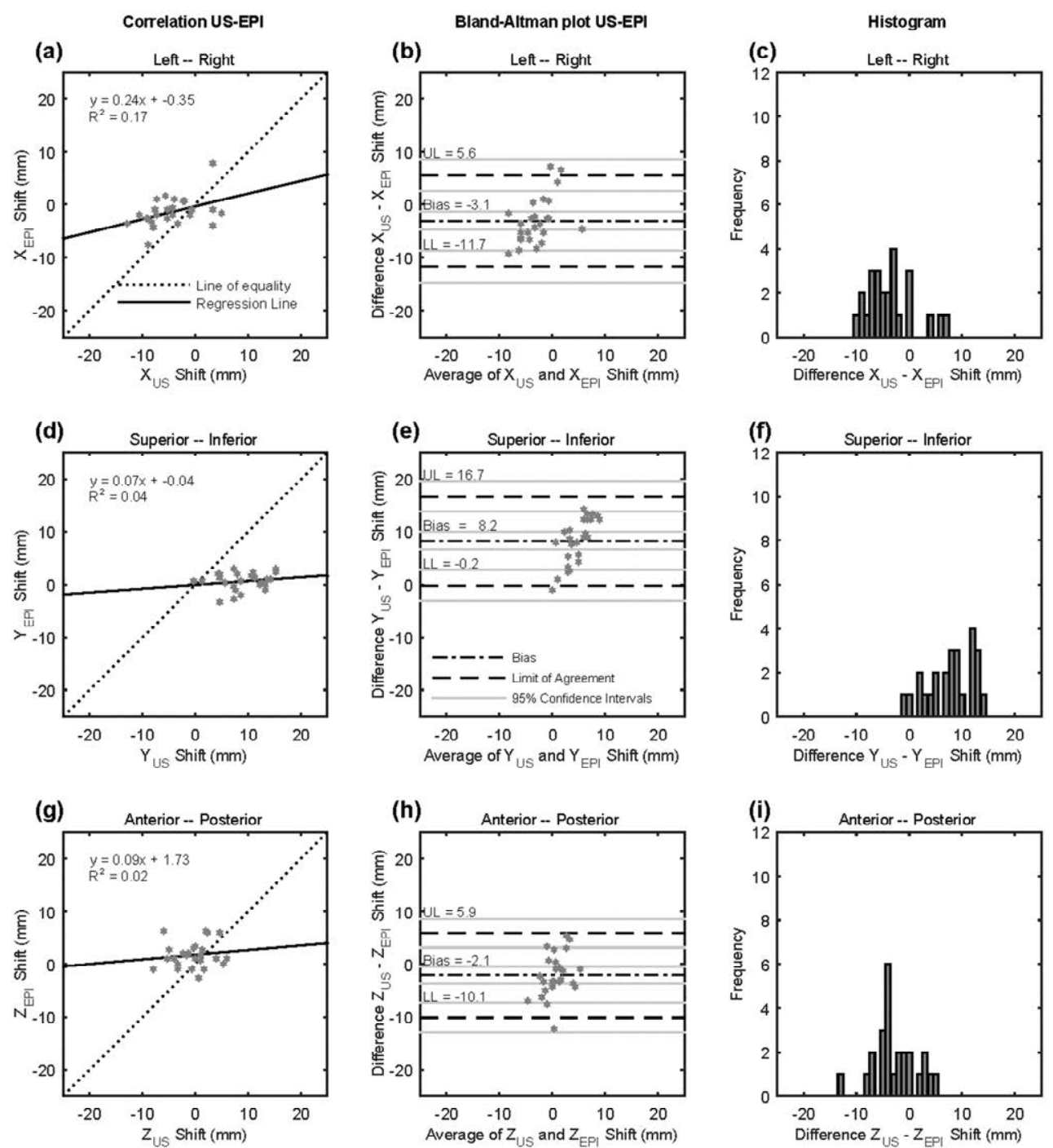

Figure C: Shifts measured with US localization vs MV portal image-based bone shifts shown in correlation plots $(a, d, g)$, Bland-Altman analysis $(b, e, h)$, and their accompanying histograms $(c, f, i)$. (Abbreviations: UL, upper limit; $L L$, lower limit).

It was observed that the systematic deviation between EPI and 3DUS image guidance in the SI direction was present for both free breathing and breath hold US scans. The mean difference for breath hold was found to be relatively large $(8.2 \pm 4.3 \mathrm{~mm})$. To investigate this deviation between 3DUS and EPI image guidance in SI direction, we analyzed the position of the diaphragm on the EPIs, because this will provide indirect information about the position of the liver during IGRT. The position of the diaphragm on the EPIs, after 
image segmentation on bony anatomy, was compared to its position on the DRR. Deviations up to $28.0 \mathrm{~mm}$ were observed. In the majority of the images the diaphragm position was found to be shifted inferiorly on the EPI compared to the DRR. A mean deviation in diaphragm position of $6.0 \mathrm{~mm}(-13.0$ to 28.0$)$ was observed. This approximately corresponds to the deviation between the US and EPI image guidance using bony anatomy.

\section{Dose evaluation}

Patient 1 is removed from the dose analysis because the dose delivery plan changed during the course of treatment. For the other patients, the evaluated dose is shown in Table C. The dose coverage of the CTV is with the US corrections better than with bone corrections, but sometimes at the cost of the right kidney and the rest of the liver. Yet, only once the dose constraints-volumes of the OARs exceeded the dose constraintsvolumes of the original plan (right kidney of patient 6), but where still within the clinical dose constraints. In figure D, as an example the DVHs are shown of patient 4.

Due to large treatment margins, even though the US corrections gave the best dose coverage to the CTV, only for patient 4 , the target would have been severely under dosed with a bone correction protocol.

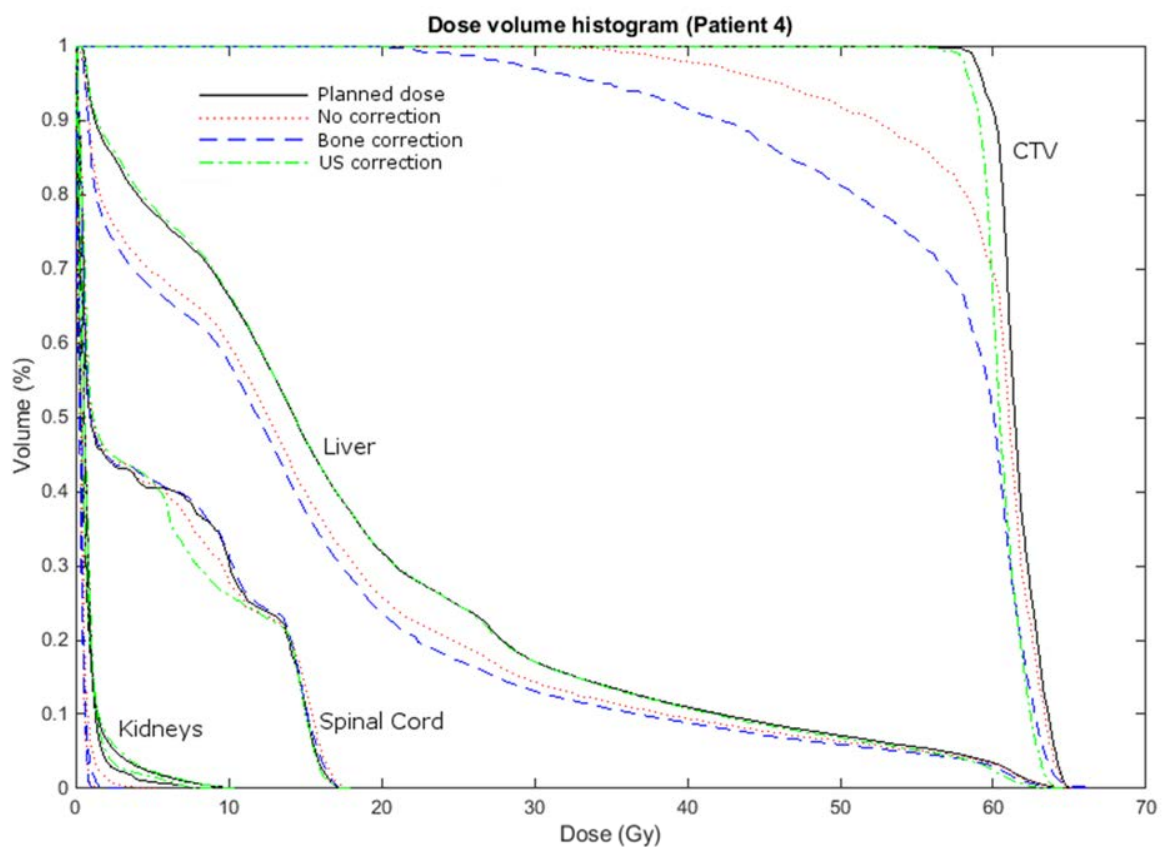

Figure D: DVH of the recalculated dose of patient 4. In black is the original planned dose. In green is the dose to the organs when an US correction shift would have been applied during treatment. In blue, the EPI shifts representing a bone correction are applied and in red is when no IGRT correction would have been applied. For all DVHs the contours of the CTV and organs liver and kidney are shifted with an US correction shift. The spinal cord, which is inside the bones, is shifted with the EPI correction shifts. 
Table C: Dose evaluation for patients 2-8. The contours of the CTV, liver and kidneys are shifted with the US shifts. For the spinal cord, the bone movement (EPI shift) was considered to be more representative. Because of different fractionation schemes, the constraints for the OAR changed from patient to patient.

\begin{tabular}{|c|c|c|c|c|c|c|c|c|}
\hline & Patient & 2 & 3 & 4 & 5 & 6 & 7 & 8 \\
\hline \multicolumn{9}{|c|}{ Planned dose } \\
\hline CTV & $\mathrm{D}_{95}(\mathrm{~Gy})$ & 47.5 & 59.3 & 59.3 & 59.1 & 58.7 & 48.5 & 57.4 \\
\hline CTV & $V_{90}$ & $100 \%$ & $100 \%$ & $100 \%$ & $100 \%$ & $100 \%$ & $100 \%$ & $100 \%$ \\
\hline Liver & & $V_{21.6} 53 \%$ & $V_{21.6} 73 \%$ & $V_{15} \quad 47 \%$ & $V_{15} 53 \%$ & $V_{21.6} 55 \%$ & $V_{21.6} 65 \%$ & $V_{24} 61 \%$ \\
\hline Left Kidney & & $V_{20} \quad 0 \%$ & $V_{20} \quad 0 \%$ & $V_{15} \quad 0 \%$ & $V_{15} \quad 0 \%$ & $V_{20} \quad 0 \%$ & $V_{20} \quad 0 \%$ & $V_{20} \quad 0 \%$ \\
\hline Right Kidney & & $V_{20} \quad 0 \%$ & $V_{20} \quad 12 \%$ & $V_{15} \quad 0 \%$ & $\mathrm{~V}_{15} \quad 14 \%$ & $V_{20} \quad 42 \%$ & $V_{20} \quad 0 \%$ & $V_{20} \quad 64 \%$ \\
\hline Right Kidney & & $\mathrm{V}_{21.6} \quad 0 \%$ & $V_{21.6} 11 \%$ & $V_{23.7} 0 \%$ & $V_{23.7} 5 \%$ & $V_{21.6} 38 \%$ & $\mathrm{~V}_{21.6} \quad 0 \%$ & $V_{24} 34 \%$ \\
\hline Spinal cord & $D_{\max }(G y)$ & 5.6 & 22.9 & 17.3 & 4.0 & 9.2 & 20.1 & 31.5 \\
\hline \multicolumn{9}{|c|}{ Bone corrections } \\
\hline CTV & $\mathrm{D}_{95}(\mathrm{~Gy})$ & 46.9 & 58.7 & 34.3 & 58.4 & 57.9 & 47.6 & 56.1 \\
\hline CTV & $V_{90}$ & $100 \%$ & $100 \%$ & $75.5 \%$ & $100 \%$ & $99.9 \%$ & $99.2 \%$ & $100 \%$ \\
\hline Liver & & $V_{21.6} 52 \%$ & $V_{21.6} 71 \%$ & $V_{15} \quad 37 \%$ & $V_{15} 54 \%$ & $V_{21.6} 55 \%$ & $V_{21.6} 59 \%$ & $V_{24} 57 \%$ \\
\hline Left Kidney & & $V_{20} \quad 0 \%$ & $V_{20} \quad 0 \%$ & $V_{15} \quad 0 \%$ & $\mathrm{~V}_{15} \quad 0 \%$ & $\mathrm{~V}_{20} \quad 0 \%$ & $\mathrm{~V}_{20} \quad 0 \%$ & $V_{20} \quad 0 \%$ \\
\hline Right Kidney & & $V_{20} \quad 0 \%$ & $v_{20} \quad 6 \%$ & $V_{15} \quad 0 \%$ & $V_{15} 13 \%$ & $V_{20} \quad 37 \%$ & $V_{20} \quad 0 \%$ & $V_{20} \quad 18 \%$ \\
\hline Right Kidney & & $V_{21.6} \quad 0 \%$ & $V_{21.6} 5 \%$ & $\mathrm{~V}_{23.7} 0 \%$ & $V_{23.7} 2 \%$ & $V_{21.6} 31 \%$ & $\mathrm{~V}_{21.6} \quad 0 \%$ & $V_{24} \quad 6 \%$ \\
\hline Spinal cord & $\mathrm{D}_{\max }(\mathrm{Gy})$ & 4.9 & 21.9 & 17.5 & 3.9 & 9.0 & 19.8 & 30.7 \\
\hline \multicolumn{9}{|c|}{ US corrections } \\
\hline CTV & $\mathrm{D}_{95}(\mathrm{~Gy})$ & 47.1 & 59.4 & 58.4 & 59.0 & 58.3 & 47.7 & 55.6 \\
\hline CTV & $V_{90}$ & $100 \%$ & $100 \%$ & $100 \%$ & $100 \%$ & $100 \%$ & $100 \%$ & $100 \%$ \\
\hline Liver & & $V_{21.6} 53 \%$ & $V_{21.6} 73 \%$ & $V_{15} \quad 47 \%$ & $V_{15} 53 \%$ & $V_{21.6} 53 \%$ & $V_{21.6} 65 \%$ & $V_{24} 59 \%$ \\
\hline Left Kidney & & $V_{20} \quad 0 \%$ & $V_{20} \quad 0 \%$ & $V_{15} \quad 0 \%$ & $\mathrm{~V}_{15} \quad 0 \%$ & $V_{20} \quad 0 \%$ & $V_{20} \quad 0 \%$ & $V_{20} \quad 0 \%$ \\
\hline Right Kidney & & $V_{20} \quad 0 \%$ & $\mathrm{~V}_{20} \quad 9 \%$ & $V_{15} \quad 0 \%$ & $\mathrm{~V}_{15} \quad 14 \%$ & $V_{20} \quad 44 \%$ & $V_{20} \quad 0 \%$ & $V_{20} \quad 63 \%$ \\
\hline Right Kidney & & $V_{21.6} \quad 0 \%$ & $V_{21.6} 8 \%$ & $V_{23.7} \quad 0 \%$ & $V_{23.7} 3 \%$ & $V_{21.6} 40 \%$ & $V_{21.6} \quad 0 \%$ & $V_{24} 30 \%$ \\
\hline Spinal cord & $D_{\max }(G y)$ & 3.5 & 20.6 & 17.9 & 4.0 & 9.3 & 18.3 & 29.2 \\
\hline
\end{tabular}

Abbreviations and symbols: CTV = clinical target volume; $D_{95}=$ Dose that at least $95 \%$ of the volume received; $D_{\max }=$ maximum received dose to the organ; $V_{90}=$ Volume that received at least a dose of $90 \mathrm{~Gy} ; \mathrm{V}_{\geq \ldots}=$ Volume that received at least the specified dose.

\section{Discussion}

In the evaluation of the shifts, a relatively large difference in the SI direction was demonstrated in the patients with US image acquisition during ABC. EPI images, required during free breathing, were compared to the DRR. The position of the liver is, however, not strongly correlated to the bone structures visible on the EPI. It is generally accepted that the mean liver position will vary around the mid-ventilation position as determined during the respiratory-correlated CT session. Treatment margins, depending on the amplitude of the breathing motion, are used to ensure that the CTV is within the planning target volume (PTV). This study, however, demonstrated a shift between EPI and US IGRT in the SI direction. The demonstrated deviation could be due to the patient's breathing pattern; 
the $50 \%$ expiration as determined on the CT scanner was not a good representation of the mean breathing phase during treatment. This can be the result of a different midventilation volume, a larger in-exhale volume or a longer expiration phase compared to the inspiration phase. A different breathing pattern will not be observed based on EPI information alone, because there is no good correlation between the bony anatomy and the position of the liver. The systematic mean deviation of $6.0 \mathrm{~mm}$ between the position of the diaphragm on the EPIs compared to its position on the DRR after image segmentation using bony anatomy, was of the same magnitude as the deviation between US and EPI image guidance. Using EPI segmentation based on the diaphragm alone is, however, doubtful. Besides, the DRR is reconstructed from the CT images created using kV images. EPIs are performed using MV images. The diaphragm consists of soft tissue and can appear differently on both image modalities. We could therefore conclude that IGRT correction shifts on MV EPI bone is not the most ideal positioning method for liver SBRT. In particular since bony anatomy is not a good surrogate for the soft-tissue of liver.

Except for the SI direction, the effect of the $A B C$, is not so clear in the comparison of the shifts. Even though the difference with EPI is in the SI direction is larger with $A B C$ than without, the uncertainty of the US procedure reduces as discussed in the first part of this chapter. Going from bone MV EPI shifts to US IGRT will have a clinically relevant effect (LoA $>5 \mathrm{~mm}$ ), but because of the reasons mentioned above, we believe this will result in an improvement in treatment. Also for the dose coverage of the CTV, the US shifts give the best results. The potential extra dose to the OAR was in all cases still within the clinical constraints.

Correction shifts bases on the bony anatomy where in 6 out of 7 patients sufficient to get good dose coverage on the CTV, but this is probably due to the large PTV margins that were used during the treatment of these patients. When using US correction shifts, the margins can probably be reduced, potently resulting in better sparing of the OAR.

\section{ACKNOWLEDGMENTS}

Without the contribution of Esther Bloemen-van Gurp, Wouter van Elmpt, Bianca Hanbeukers and Mark Podesta this short report would not have been possible.

\section{REFERENCES}

1 E. Bloemen-van Gurp, S. van der Meer, J. Hendry, et al., "Active breathing control in combination with ultrasound imaging: a feasibility study of image guidance in stereotactic body radiation therapy of liver lesions", 2013 International journal of radiation oncology, biology, physics (85:1096-1102)

2 J.M. Bland, D.G. Altman, "Statistical methods for assessing agreement between two methods of clinical measurement", 1986 Lancet (1:307-310) 

TOWARDS ADAPTIVE RADIOTHERAPY 



\section{CHAPTER}

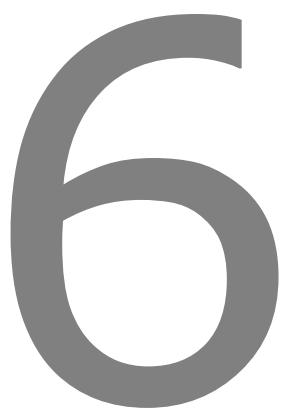

Simulation of pseudo-CT images based on deformable image registration of ultrasound images: a proof of concept for transabdominal ultrasound imaging of the prostate during radiotherapy

Skadi van der Meer*, Saskia M. Camps*, Wouter J.C. van Elmpt, Mark Podesta, Pedro Gomes Sanches, Ben G.L. Vanneste, Davide Fontanarosa and Frank Verhaegen

*These authors contributed equally to this work.

Submitted to: Medical Physics, 2015. 


\begin{abstract}
Purpose: Imaging of patient anatomy during treatment is a necessity for position verification, and for adaptive radiotherapy based on daily dose recalculation. Ultrasound image guided radiotherapy systems are currently available to collect ultrasound images at the simulation stage, co-registered with the simulation computed tomography, and during all treatment fractions. We hypothesize that a deformation field derived from ultrasound based deformable image registration can be used to create a daily pseudo computed tomography image that is more representative of the patients geometry during treatment than the computed tomography acquired at simulation stage.

Methods: The three prostate patients, considered to evaluate this hypothesis, had coregistered computed tomography and ultrasound scans on various days. Deformation fields were computed between two ultrasound images and then applied to the corresponding simulation computed tomography scan to yield a new deformed computed tomography scan. The original treatment plans were used to recalculate dose distributions in the simulation, deformed and ground truth computed tomography images to compare dice coefficients on computed tomography delineations and gamma index evaluations on both the Hounsfield units and the dose.
\end{abstract}

Results: In the majority, deformation did improve the results for all three evaluation methods. The change in gamma failure for dose (3\%, 3 millimeter) ranged from an improvement of $11.2 \%$ in the prostate volume to a deterioration of $1.3 \%$ in the prostate and bladder. The change in gamma failure for the computed tomography images (50 Hounsfield units, 3 millimeter) ranged from an improvement of $20.5 \%$ in the anus and rectum to a deterioration of $3.2 \%$ in the prostate.

Conclusions: This new technique may generate pseudo computed tomography images that are more representative of the actual patient anatomy than the simulation computed tomography scan. 


\section{INTRODUCTION}

Image guidance has become an essential part of radiotherapy (RT) treatment to allow for safe delivery of radiation doses. Image guided RT (IGRT) is often performed for several or all treatment fractions to position the patient correctly. Beyond the aim of image guidance, the availability of daily imaging also allows for the possibility of adaptive RT (ART) ${ }^{1,2}$. The goal of ART is to improve RT treatment by systematically monitoring dose discrepancies and incorporating them to re-optimize the treatment plan. Normally only the planning computed tomography (CT) image, acquired at simulation stage, is available for the dose calculation, but both inter- and intra-fraction patient anatomy motion and changes (like tumor shrinkage, nodal volume changes and weight loss) may alter the dose distribution ${ }^{3-}$ 6 . In ART, the anatomy from the planning CT is updated by the anatomy from the daily imaging, acquired during the IGRT workflow to monitor dose distribution and if necessary adapt the treatment plan.

CT scanners are usually not available in the treatment room. Instead, cone beam computed tomography (CBCT) can be used for dose calculations either directly ${ }^{7-10}$, or indirectly with deformable image registration (DIR) ${ }^{11,12}$ even though they offer a lower image quality when compared to CT scanners. In some studies, using the CBCT directly for dose calculations, the inaccuracies in the Hounsfield units (HU) are large enough to result in clinically relevant dose errors ${ }^{13-15}$.

A 3D Ultrasound (US) IGRT system can acquire volumetric, high-contrast soft-tissue images non-invasively on a daily basis without using ionizing radiation (figure 1). Intramodality US-systems use next to the US images acquired daily during the treatment sessions $\left(U S_{\mathrm{tx}}\right)$, a planning $\mathrm{CT}\left(\mathrm{CT}_{\text {sim }}\right)$ and $\mathrm{US}\left(\mathrm{US}_{\text {sim }}\right)$ image acquired during the simulation stage.

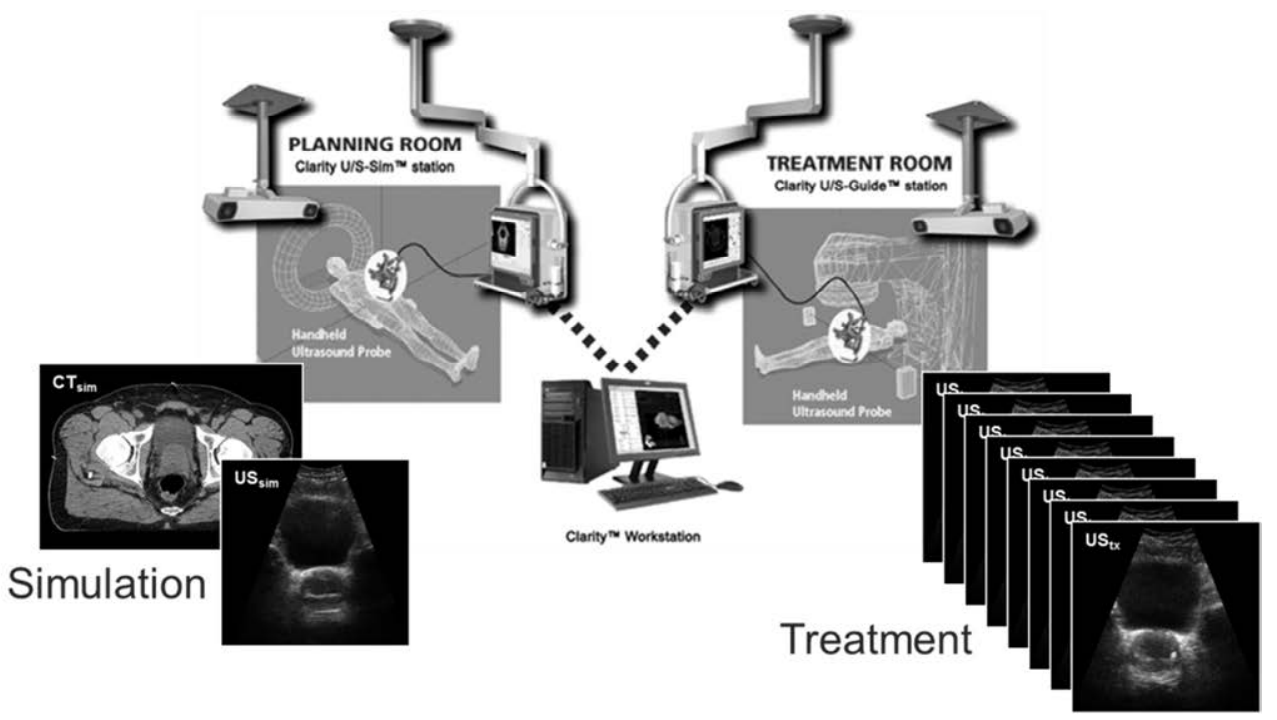

Figure 1: Workflow of acquisition of $C T_{\text {sim, }} \cup S_{\text {sim }}$ and $U S_{t x}$ images (Clarity US system; Elekta). (Figure adapted from Elekta with their permission) 
The main concept in this paper is that a pseudo-CT image can be created based on $\mathrm{CT}_{\text {sim }}$ using a deformation field calculated between the $\mathrm{US}_{\text {sim }}$ and $\mathrm{US}_{\mathrm{tx}}$. We hypothesize that the deformed pseudo-CT image $\left(\mathrm{CT}_{\mathrm{ps}}\right)$ created this way is a better representation of the patient's anatomy than the planning $\mathrm{CT}_{\text {sim }}$ during treatment delivery.

\section{MATERIALS AND METHODS}

\section{The concept}

The approach used to represent the patient geometry during treatment delivery, is that the daily acquired US images are used to create a pseudo $\mathrm{CT}_{\mathrm{ps}}$ image with DIR. To generate the daily $\mathrm{CT}_{\mathrm{ps}}$, a deformation field calculated between the $\mathrm{US}_{\text {sim }}$ and the daily $\mathrm{US}_{\mathrm{tx}}$ was applied to $\mathrm{CT}_{\text {sim }}$ (figure 2).

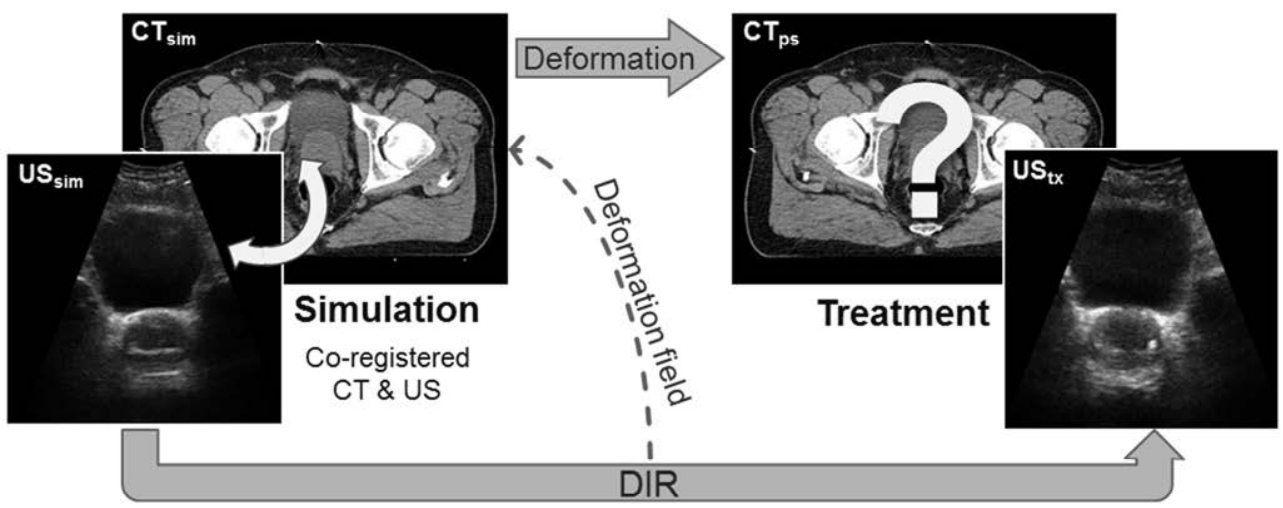

Figure 2: Deformable image registration (DIR) is computed between the two US images (US sim \& US $S_{t x}$ ) and then applied to $C T_{\text {sim; }}$; new pseudo $C T_{p s}$ is obtained. The question is whether this $C T_{p s}$ is indeed representative for the patient anatomy during treatment.

\section{Patient scans}

Clinical examples with multiple co-registered US-CT combinations at the simulation stage (instead of the treatment stage), were used to validate the concept. Three prostate cancer patients from a previous study ${ }^{16}$ were used who, (due to clinical reasons) in addition to the daily $\mathrm{US}_{\mathrm{tx}}$ imaging, underwent extra $\mathrm{CT}$ and US imaging $\left(\mathrm{CT}_{\text {sim }}\right.$ and $\left.\mathrm{US}_{\text {sim }}\right)$ on several days. In the normal clinical workflow, these extra CT and US images are not acquired. The extra $\mathrm{CT}$ scans were used as ground truth scans $\left(\mathrm{CT}_{\mathrm{GT}}\right)$ to which the derived $\mathrm{CT}_{\mathrm{ps}}$ scans can be compared. In Table 1, the method used to calculate and evaluate the result from the deformations is described.

The co-registered CT-US images were acquired at two time points for patients 1 and 2 ( 3 and 1 weeks apart, respectively). Acquisitions for patient 3 were made for five time 
points where the first two were 2 weeks apart and the following three time points were one week apart.

All co-registered US-CT combinations were acquired in the CT-room with the patient's external skin markers positioned along the room lasers. The US scans (Clarity system; Elekta, Stockholm, Sweden. Probe type C5-2/60, center frequency 3.5 MHz, Sonix Series; Ultrasonix Medical Corporation, Richmond, BC, Canada) were performed transabdominally immediately before or after the CT-scan (SOMATOM Sensation Open, Syngo CT 2006A, Siemens, Germany; voxels: $1 \times 1 \mathrm{~mm}^{2} \times 3 \mathrm{~mm}$ slice thickness). Both scans were performed in the same supine patient position, stabilized with knee fix and foot support (Combifix, Civco Medical Solutions, Kalona, lowa, USA), resulting in a correct automatic fusion of the US and CT images ${ }^{17}$.

In all US images, the prostate was delineated. All CT images had delineations of the body contour, prostate, seminal vesiculae (SV, except for patient 3), anus, rectum and bladder (except for patient 1).

Table 1: Overview of the US-CT combinations used to calculate and evaluate US-based deformable image registration. Patient $3 a-d$ differ in the second US-CT pair that was used to test the method.

\begin{tabular}{cccccc}
\hline Patient & Used as US sim $_{\text {s }}$ & Used as US $\mathbf{S}_{\mathbf{t x}}$ & Used as $\mathbf{C T}_{\text {sim }}$ & $\begin{array}{c}\text { Used as } \\
\mathbf{C T}_{\mathrm{GT}}\end{array}$ \\
\hline 1 & & $\mathrm{US}_{1}$ & $\mathrm{US}_{2}$ & $\mathrm{CT}_{1}$ & $\mathrm{CT}_{2}$ \\
2 & & $\mathrm{US}_{1}$ & $\mathrm{US}_{2}$ & $\mathrm{CT}_{1}$ & $\mathrm{CT}_{2}$ \\
3 & $\mathrm{a}$ & $\mathrm{US}_{1}$ & $\mathrm{US}_{2}$ & $\mathrm{CT}_{1}$ & $\mathrm{CT}_{2}$ \\
3 & $\mathrm{~b}$ & $\mathrm{US}_{1}$ & $\mathrm{US}_{3}$ & $\mathrm{CT}_{1}$ & $\mathrm{CT}_{3}$ \\
3 & $\mathrm{c}$ & $\mathrm{US}_{1}$ & $\mathrm{US}_{4}$ & $\mathrm{CT}_{1}$ & $\mathrm{CT}_{4}$ \\
3 & $\mathrm{~d}$ & $\mathrm{US}_{1}$ & $\mathrm{US}_{5}$ & $\mathrm{CT}_{1}$ & $\mathrm{CT}_{5}$ \\
\hline
\end{tabular}

Abbreviations: $\mathrm{CT}$, computed tomography; $\mathrm{CT}_{\mathrm{GT}}$, ground truth $\mathrm{CT}$ acquired at the same time as the US image used as $\mathrm{US}_{\mathrm{tx}} ; \mathrm{CT}_{\text {sim }}$, reference/planning $\mathrm{CT}$, acquired at the time of simulation; $\mathrm{GT}$, ground truth; US, ultrasound; US $_{\text {sim, }}$, reference/planning US, acquired at the time of CT simulation; $\mathrm{US}_{\mathrm{tx}}$, US images daily acquired at the treatment stage;

\section{Deformation}

The deformation fields were calculated using a DIR algorithm (B-spline method from Elastix; Utrecht, the Netherlands) ${ }^{18,19}$ with a rigidity penalty ${ }^{20}$ on a mask based on a bone segmentation of $\mathrm{CT}_{\text {sim }}$. Deformation fields were computed between the pairs of US images (moving: $\mathrm{US}_{\text {sim }}$ and fixed: $\mathrm{US}_{\mathrm{tx}}$ ) and then applied to $\mathrm{CT}_{\text {sim. }}$. The deformation field calculation was based on the overlapping parts of the US images (ROI: $U S_{s i m} \cap U S_{t x}$ ), but will propagate further through the image (figure 3). Even though the deformation field extrapolates through the rest of the image, the rigidity penalty on the bone segmentation remains intact to prevent the bones from deforming. Prior to the DIR calculation all the volumes were resampled to the same voxel size and image dimensions per patient. Preprocessing (including masks and ROI construction) was performed in Matlab (MathWorks Inc., Natick, $\mathrm{MA})$. 

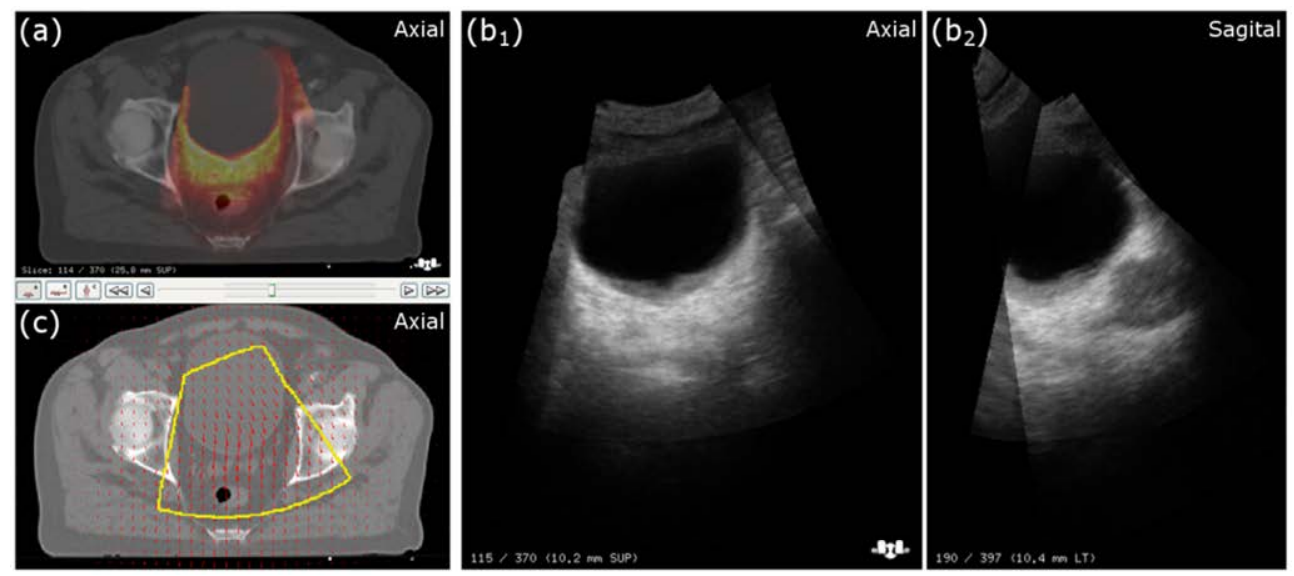

Figure 3: Example of overlap between CT (grey) \& US (color) (a), and between two US images (b) of patient 1. US based DIR can only be performed on the area where both CT and US information (of both $U S_{s i m}$ and $U S_{t x}$ ) is available. In this example only the prostate and its surrounding tissue e.g. a part of the bladder are present in both US images. In panel (c) only the overlapping area of both US images (yellow contour) contains information where the deformation field (2D representation with red arrows) is based on. The field propagates further beyond this border.

The patients were in the same position with the body markers aligned to lasers, during the different US-CT combinations. Therefore no rigid transformation was applied beforehand, in particular because the prostate can shift within the body, instead of indicating a full body shift ${ }^{21}$.

In total five different parameter sets for the DIR were tested (Supplementary materials $A$ ). Three of those were based on the intensities of the US images using a normalizedcorrelation metric (parameter set A: 10, B: 50 and C: 100 iterations). The other two sets were based only on the prostate contours of the US images, using a mean-squares metric on a binary mask of the delineated prostate volumes (parameter set D: 100 and E: 300 iterations). The deformation fields applied to a $\mathrm{CT}_{\text {sim }}$ were also used to deform the corresponding CT delineations.

\section{Evaluation of the deformation}

The created $\mathrm{CT}_{\mathrm{ps}}$ and the deformed $\mathrm{CT}$ delineations were then compared to the ground truth, i.e. the corresponding $\mathrm{CT}_{\mathrm{GT}}$ and its delineations. The contours were evaluated using the dice coefficients $(D C=(2|X \cap Y|) /(|X|+|Y|))$. A DC ratio of 1 indicates complete overlap, while 0 indicates no overlap. The $\mathrm{CT}_{\text {sim }}$ and $\mathrm{CT}_{\mathrm{ps}}$ images were compared to $\mathrm{CT}_{\mathrm{GT}}$ using a gamma $(\gamma)$ evaluation ${ }^{22,23}$, normally used for dose evaluations. In this case the HU were evaluated instead of the dose $\left(\gamma_{\mathrm{CT}}\right)$. The $\gamma$ values were calculated using an in-house developed method ${ }^{24,25}$ using Matlab and $\mathrm{C}^{++}$. The used method allows the sign of the $\gamma$ value to indicate whether an overdose $(\gamma>0)$ or underdose $(\gamma<0)$ is found for each voxel ${ }^{25}$. In this case, because we don't evaluate the dose but $\mathrm{HU}$, a $\gamma>0$ means that the $\mathrm{HU}$ is relatively higher than the reference and $\gamma<0$ means that the $H U$ is relatively lower. $A$ value $|\gamma|>1$ in a voxel indicates that the voxel fails to meet the acceptance criteria, in this case a $50 \mathrm{HU}$ 
voxel intensity difference and a $3 \mathrm{~mm}$ distance to agreement. (The $50 \mathrm{HU}$ is a conservative measure based on that for typical radiotherapy beams; to produce a $1 \%$ error in dosimetry would require errors of over $8 \%$ in bone electron density ${ }^{26}$ and hence $\mathrm{HU}$. The $3 \mathrm{~mm}$ distance to agreement is a commonly used criterion in dosimetry ${ }^{23}$. The percentages of the volume with a $\left|\gamma_{\mathrm{CT}}\right|>1$ within the contours 'intersection body contours', 'prostate', 'anus \& rectum' and 'bladder' were reported. The percentages of gamma failure and DC evaluations are reported using the contours of the $\mathrm{CT}_{\mathrm{GT}}$, except for the intersection body contours' which is the overlapping part of the body contours of both $\mathrm{CT}_{\operatorname{sim}}$ and $\mathrm{CT}_{\mathrm{GT}}$.

\section{Dose calculation and evaluation}

Dose distributions were obtained by recalculating the original treatment plans (5-beam IMRT plans; XiO CMS 4.51, Elekta, Stockholm, Sweden) designed on the planning $\mathrm{CT}_{\text {sim }}$, on the $\mathrm{CT}_{\text {sim }}, \mathrm{CT}_{\mathrm{ps}}$ and $\mathrm{CT}_{\mathrm{GT}}$ scans. For this, an in-house developed software was used, based on Monte Carlo simulation using the XVMC code ${ }^{27,28}$. Dose distributions on the $\mathrm{CT}_{\text {sim }}$ and $\mathrm{CT}_{\mathrm{ps}}$ images were compared to the dose on $\mathrm{CT}_{\mathrm{GT}}$ using a $\gamma$ evaluation $\left(\nu_{\text {Dose }}\right)^{22}$, with acceptance criteria of $3 \%$ dose difference and $3 \mathrm{~mm}$ distance to agreement. Again the percentage of the volume with a $\left|\gamma_{\text {Dose }}\right|>1$ within the contours intersection body contours', 'prostate', 'anus \& rectum' and 'bladder' was reported.

\section{RESULTS}

In most cases, deformation did improve the results according to all evaluation methods, although these improvements were in some cases very small or even negligible. Only for patient 1 there was a large improvement (more than 10\% decrease in the volume with $\left.\left|\gamma_{\text {Dose }}\right|>1\right)$ in the dose of the prostate when the intensity based normalized-correlation metric with 100 iterations (parameter set C) was used (Table 2). In figure 4, an example is given for patient 1 using parameter set $C$. In the second column the overlap of the prostate and anus \& rectum contours is shown. DC increased by 0.26 when the deformations were used. The third and fourth column show the $\gamma_{\mathrm{CT}}$ and $\gamma_{\text {Dose }}$ values. In the overlapping body contours, the percentage of $\gamma_{C T}$ failure decreased by $1.7 \%$ in volume. For the prostate and anus \& rectum contours, there was a $\gamma_{\mathrm{CT}}$ failure decrease of $8.9 \%$ and $8.4 \%$, respectively. For the dose, the volume percentage of $\gamma_{\text {dose }}$ failure decreased by $11.2 \%$ in volume for the prostate. Yet the percentage of $\gamma_{\text {dose }}$ failure decreased by only $0.6 \%$ and $0.1 \%$ for the overlapping body contours and anus \& rectum, respectively. The graphs of these results for patient 1 are, together with the results for the other 4 parameter sets, shown in figure 5 . (In the supplementary materials B, figure $B$, an overview of all patients can be found.) For the same parameter set, patient $3 d$ had a $20 \%$ decrease in the volume with $\left|\gamma_{\mathrm{CT}}\right|>1$ for the anus \& rectum (Table B in supplementary materials B), but only a small volume reduction of $0.13 \%$ appeared in $\left|\gamma_{\text {Dose }}\right|>1$ because the difference was already very small $(0.52 \%)$ initially (Table 2$)$. 

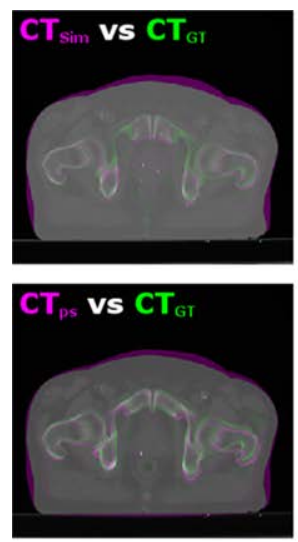

CTs
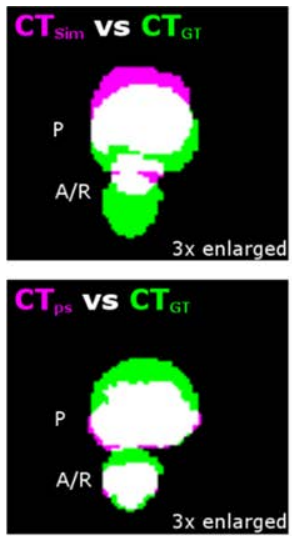

Overlap of Prostate and Anus/Rectum
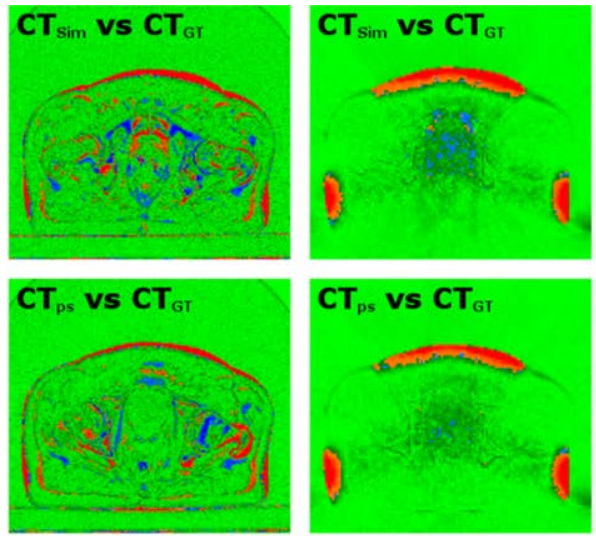

$\mathrm{Y}_{\mathrm{cr}}(50 \mathrm{HU}, 3 \mathrm{~mm})$

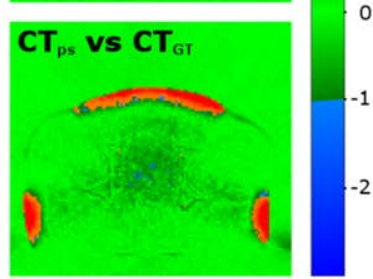

$Y_{\text {Dose }}(3 \%, 3 \mathrm{~mm}) \quad \mathrm{Y}$

Figure 4: Results for patient 1 (parameter set $C$ ). In the first colums the $C T_{\text {sim }}$ and $C T_{p s}$ (in pink) are compared to $C T_{G T}$ (in green). In the second column, the contours of prostate (P) and anus/rectum $(A / R)$ are compared. When the images are greyscale (column 1) or white (column 2) there is overlap between the compared images. The third and fourth columns show the $\gamma_{C T}$ (column 3) and $\gamma_{\text {Dose }}$ (column 4). In green, the $\gamma$ values are between -1 and 1 . In red and blue are the voxels in which the $\gamma$ failed to meet the criteria of $(5 \mathrm{OHU}, 3 \mathrm{~mm})$ for the CT values and $(3 \%, 3 \mathrm{~mm})$ for the dose. For column 4 , the areas where there is an underdosages compared to $C T_{G T}\left(V_{\text {Dose }}<-1\right)$ are shown in blue. In red there is an overdosage compared to $C T_{G T}\left(V_{\text {Dose }}>1\right)$.

(a) Dice coefficient

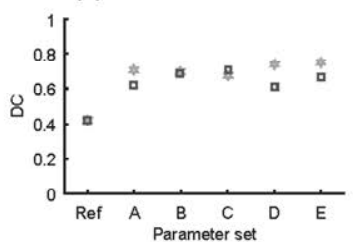

(b) Gamma on CT values

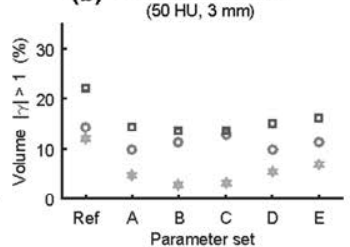

(c) Gamma on dose

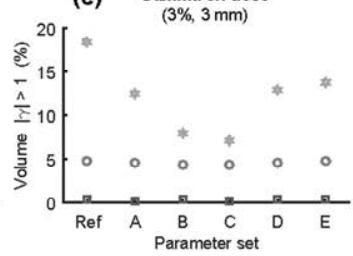

Patient 1

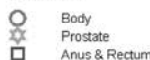

Figure 5: Results for all five parameter sets used on patient 1. a) The DC improved for all parameter sets. For prostate the best results were obtained with parameter set $E$, for anus/rectum it was $C$. b) For the gamma evaluation on the CT values the volume percentage of the gamma failure (/ $\boldsymbol{V}_{\mathrm{CT}(5 \mathrm{OHU}, 3}$ $\mathrm{mm}_{\mathrm{m}} />1$ ) is plotted. The best parameter set for the body contour was parameter set $D$, for the two organs it was parameter set $B$. c) For the gamma evaluation on the dose the percentage of a gamma failure $\left(\left|\gamma_{\text {Dose }(3 \%, 3 \mathrm{~mm})}\right|>1\right)$ is plotted. Here parameter set $C$ gave the best results. 
Looking at all patient cases (Table B1 and figure $B(a, d, g, j, m, q)$ in supplementary materials $B$ ), DC increased the most for the two contour-based parameter sets ( $D$ and $E$ ). (Parameter set $\mathrm{E}$ with 300 iterations did not succeed in the deformation of patient 2 because there was a too small overlapping volume. Therefore not enough voxels could be mapped and the registration failed to find a solution.) Only for patient 3a none of the parameter sets gave an improvement for any of the contours. Overall, the maximum changes in DC for the intensity based normalized-correlation parameter sets were a decrease of -0.46 or an improvement of +0.29 . For the contour based parameter sets, these were -0.22 and +0.38 .

For the changes in CT HU values, the percentage of the volume with a $\left|\gamma_{\mathrm{CT}(5 \mathrm{OHU}, 3 \mathrm{~mm})}\right|>$ 1 is shown in the supplementary materials (Table B2 and figure B (b,e,h,k,n,q)). A maximum improvement was seen of $20.5 \%$ ( $14.6 \%$ for contour based) and the poorest results gave an increase of $3.2 \%$ (2.2\% for contour based) in the volume with $\left|\gamma_{\mathrm{CT}(5 \mathrm{OHU}, 3 \mathrm{~mm})}\right|>1$.

In Table 2, the dose differences $\left(\left|\gamma_{\text {Dose }(3 \%, 3 \mathrm{~mm})}\right|>1\right)$ are shown (the corresponding graphs are displayed in figure $B(c, f, i, l, o, r)$ in supplementary materials $B)$. For the dose a maximum improvement of $11.2 \%$ (5.3\% for contour based) was seen and the poorest results yielded an increase of $1.3 \%$ ( $0.5 \%$ for contour based) in the volume with $\mid \gamma_{\text {Dose }(3 \%, 3}$ $\mathrm{mm}) \mid>1$.

Looking at all results for all patients, when there was an improvement, the contour parameter set ( $D, 100$ iterations) seemed to give an improvement in most cases, yet it was not always the best one. 
Table 2: Gamma evaluation on the dose where the doses on the $C T_{\text {sim }}$ (Ref) and $C T_{p s}$ images (parameter set A-E) were compared to the dose on $C T_{G T}$. Shown is the volume percentage of a gamma failure $\left(V_{\text {Dose }}(3 \%, 3 \mathrm{~mm})>1\right)$ of the different contours. The cases when the gamma failure increased compared to the reference situation, i.e. the deformations made it worse, are underlined.

\begin{tabular}{|c|c|c|c|c|c|c|c|}
\hline \multirow[b]{2}{*}{ Patient } & \multirow[b]{2}{*}{ Contour } & \multicolumn{5}{|c|}{ Volume $|\gamma \operatorname{Dose}(3 \%, 3 \mathrm{~mm})|>1$ (\%) } & \multirow[b]{2}{*}{ E } \\
\hline & & Ref & A & B & C & D & \\
\hline \multirow[t]{3}{*}{1} & Body $(\cap)$ & 4.9 & 4.6 & 4.4 & $4.3^{*}$ & 4.6 & 4.7 \\
\hline & Prostate & 18.3 & 12.4 & 8.1 & $7.1^{*}$ & 13.0 & 13.7 \\
\hline & Anus \& Rectum & 0.2 & $0.2^{*}$ & 0.2 & $0.2^{*}$ & 0.2 & $\underline{0.4}$ \\
\hline \multirow[t]{4}{*}{2} & Body $(\cap)$ & 2.8 & 2.8 & 2.8 & 2.8 & $2.6^{*}$ & - \\
\hline & Prostate & 1.6 & $\underline{2.2}$ & $\underline{2.8}$ & $\underline{2.9}$ & $1.4^{*}$ & - \\
\hline & Anus \& Rectum & 0.8 & $0.2^{*}$ & 0.5 & 0.7 & $\underline{0.9}$ & - \\
\hline & Bladder & 0.0 & $\underline{0.3}$ & $\underline{0.7}$ & $\underline{1.0}$ & 0.0 & - \\
\hline \multirow[t]{4}{*}{ 3a } & Body $(\cap)$ & 0.6 & $0.6^{*}$ & $\underline{0.6}$ & $\underline{0.6}$ & 0.6 & 0.6 \\
\hline & Prostate & 2.3 & 2.0 & $1.6^{*}$ & 1.9 & 2.1 & 1.9 \\
\hline & Anus \& Rectum & 4.2 & $\underline{4.6}$ & $\underline{4.3}$ & $\underline{4.4}$ & $\underline{4.4}$ & $\underline{4.7}$ \\
\hline & Bladder & 0.0 & 0.0 & 0.0 & 0.0 & 0.0 & 0.0 \\
\hline \multirow[t]{4}{*}{$3 b$} & Body $(n)$ & 0.9 & 0.8 & 0.8 & 0.8 & 0.5 & $0.5^{*}$ \\
\hline & Prostate & 3.8 & 3.5 & 2.7 & 3.2 & $1.8^{*}$ & 2.2 \\
\hline & Anus \& Rectum & 0.0 & $0.0^{*}$ & 0.0 & $0.0^{*}$ & 0.0 & $0.0^{*}$ \\
\hline & Bladder & 0.0 & $\underline{0.0}$ & 0.0 & $\underline{0.0}$ & 0.0 & 0.0 \\
\hline \multirow[t]{4}{*}{$3 c$} & Body $(\cap)$ & 1.5 & $\underline{1.6}$ & $\underline{1.8}$ & $\underline{1.7}$ & 1.1 & $1.1^{*}$ \\
\hline & Prostate & 4.1 & $3.3^{*}$ & 3.5 & $\underline{4.2}$ & 3.4 & 3.8 \\
\hline & Anus \& Rectum & 0.0 & $0.0^{*}$ & $0.0^{*}$ & $0.0^{*}$ & $0.0^{*}$ & $0.0^{*}$ \\
\hline & Bladder & 0.0 & $0.0^{*}$ & $\underline{1.3}$ & $\underline{1.0}$ & $0.0^{*}$ & $0.0^{*}$ \\
\hline \multirow[t]{4}{*}{$3 d$} & Body $(\cap)$ & 2.0 & 2.0 & 1.9 & 1.8 & $1.5^{*}$ & 1.5 \\
\hline & Prostate & 10.3 & 9.9 & 9.5 & 8.7 & 6.9 & $6.5^{*}$ \\
\hline & Anus \& Rectum & 0.5 & 0.2 & $\underline{0.6}$ & 0.4 & $0.1^{*}$ & 0.2 \\
\hline & Bladder & 0.3 & 0.2 & 0.1 & 0.0 & $0.0^{*}$ & $0.0^{*}$ \\
\hline
\end{tabular}

* Parameter set with the best improvement.

Abbreviations: $\gamma_{\text {Dose, }}$ gamma evaluated on the dose; $n$, intersection of the body contours of $\mathrm{CT}_{\text {sim }}$ and $\mathrm{CT}_{\mathrm{GT}} ; \mathrm{GT}$, ground truth; Ref, reference situation of $\mathrm{CT}_{\text {sim }}$ compared to $\mathrm{CT}_{\mathrm{GT}}$;

\section{DISCUSSION}

We have evaluated the impact of applying US-derived tissue deformations to approximate $\mathrm{CT}$ images to the real anatomical organ position of prostate patients during radiation 
therapy. A similar workflow was presented by Pennec et $a .^{29}$ for brain surgery applications. Pre-operative magnetic resonance (MR) imaging and US were acquired and subsequently intra-operative US scans were used to create pseudo-MR images of the brain. This resulted in acceptable representations of brain anatomy during surgery. To our knowledge, this is the first time such a method is used for RT applications.

In this study, patients 1 and $3 d$ would have benefited most from the deformations (> $3 \%$ volume decrease for the volume with a $\left.\left|\gamma_{\text {Dose }}\right|>1\right)$. In addition, the difference in dose between $\mathrm{CT}_{\text {sim }}$ and $\mathrm{CT}_{\mathrm{GT}}$ was there also the largest ( $>10 \%$ volume with a $\left.\left|\gamma_{\text {Dose }}\right|>1\right)$. For the other patient cases the improvements were not clinically relevant. Ideally, one should be able to evaluate beforehand which patients would benefit from applying the deformations. The only metric available before deformation and without a $\mathrm{CT}_{\mathrm{GT}}$ is the $\mathrm{DC}$ of the prostate contours on the two US images US $_{\text {sim }}$ and US $\mathrm{S}_{\mathrm{tx}}$ (Table B1 in supplementary materials $B$ ). For patient 1 and $3 d$ the $D C$ was 0.43 and 0.28 and for all others it ranged from $0.42-0.70$. From this study with a limited number of patients, no correlation could be extracted between the $D C$ and the volume changes in $\left|\gamma_{\text {Dose }}\right|>1$ for the prostate $\left(R^{2}\right.$ was 0.3 and 0.2 for respectively the parameter set $D$ and the individual best parameter set) (figure 6). The correlation between the DC of the US prostate contours ( $U S_{\text {sim }}$ and US $S_{t x}$ ) and the volume change in $\left|\gamma_{\mathrm{CT}}\right|>1$ of the anus \& rectum contour was much stronger $\left(R^{2}\right.$ was 0.8 and 0.7 for respectively the parameter set $D$ and the individual best parameter set). There seems to be a trend that the patients with the largest geometric changes benefit most from deformations, but a future study with a larger image database will be necessary to validate the predictive power of this DC parameter to get a clearer indication when it is worthwhile to perform DIR.
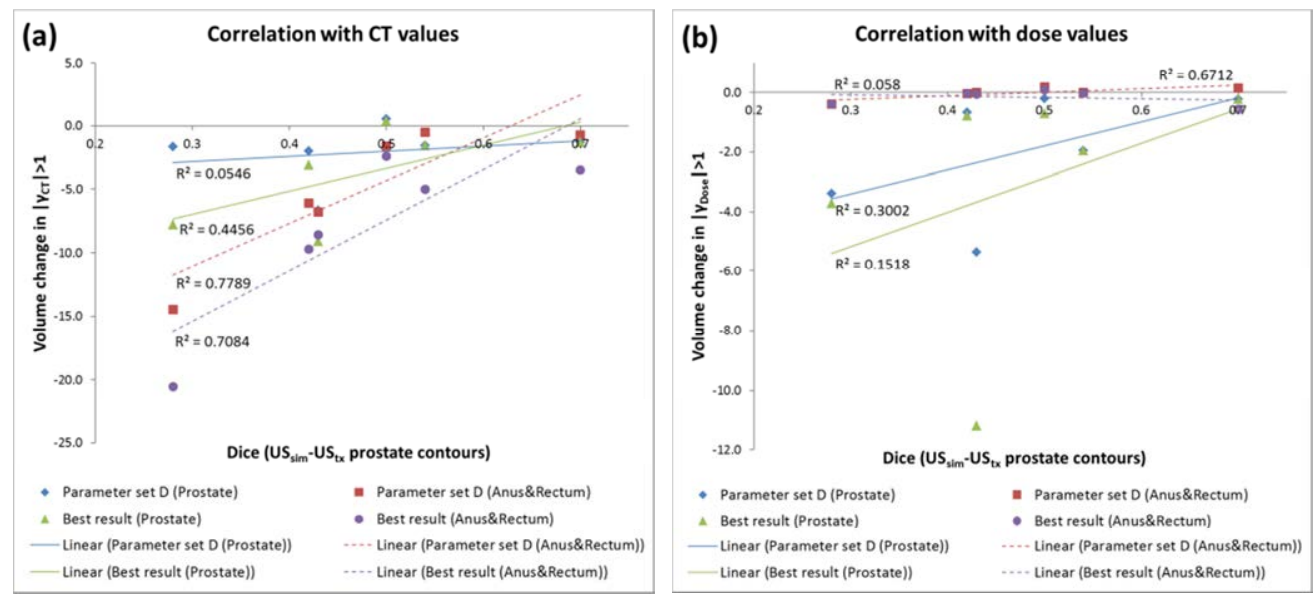

Figure 6: (a) The correlation between the dice coefficients (DC) of the US prostate contours (US $S_{\text {sim }}$ and $\left.U S_{t x}\right)$ and the volume change in $\left|\gamma_{C T}\right|>1$ of prostate (solid line) and anus \& rectum (dotted line) contours. (b) The correlation between the DC of the US prostate contours (US $S_{s i m}$ and $\left.U S_{t x}\right)$ and the volume change in $\left|Y_{\text {Dose }}\right|>1$. Both graphs contain the results of the individual best parameter set (indicated by * in Table 2 and B2) and parameter set $D$. 
Besides a larger database to perform statistics, such a database could be used to find an optimal metric and parameter set for the DIR. For this proof-of-principle study, two deformation metrics were used and only the number of iterations varied. Optimization of the metrics and parameter set may improve the results. In the current study, the results of the evaluation methods were rarely in agreement. Even between the CT and dose values there were some differences due to the cumulative effect of the dose along the beam path. The differences between change in $\gamma_{\mathrm{CT}}$ and $\gamma_{\text {Dose }}$ are caused by the fact that the dose in the organs is not only dependent on the local $\mathrm{HU}$, but also on the $\mathrm{HU}$ along the beam path. The best evaluation method is dependent on the purpose; the evaluation of the best parameter set should therefore always be assessed with the correct evaluation method. In case of ART, this could be $\gamma_{\text {Dose }(3 \%, 3 \mathrm{~mm}) \text {. }}$

A limitation of an US-based deformation field is that the volume of the CT on which one can directly calculate the deformation field is limited to the volume of the US data available (figure 3). The deformation field propagates further, but this is not based on image data and is therefore maybe less reliable. For patient 2, a small overlap of US volumes resulted in a failure in parameter set $\mathrm{E}$. Standardization of scanning, so that at least the complete prostate is visible, the US volume overlap is maximal, and US images with larger fields of view may improve the results. Transperineal scanning with a larger image sector or perhaps even fusion of multiple US scans from different directions can extend the field of view. However the US image will never completely overlap the CT image, therefore part of the deformation field will still be based on only an extrapolated deformation field.

Another reason why it is important to have standardization of the US scanning is that, just like with the IGRT usage of the US images, it is important to have reproducible US images. In particular the probe pressure ${ }^{16,30}$ and speed-of-sound aberration ${ }^{31}$ along the imaging beam should be comparable. One cannot distinguish between the US imaging dependent changes caused by non-standardized procedures or a real anatomy changes. Therefore it is best to prevent them, or correct ${ }^{32-35}$ for them before the DIR procedure.

Validation of the DIR methods in general is also still necessary to reliably perform DIR for ART. Different deformation algorithms lead to different results, therefore more research is necessary.

\section{CONCLUSIONS}

It was possible to generate a pseudo- $\mathrm{CT}_{\mathrm{ps}}$ with the use of DIR based on US imaging which was more representative of $\mathrm{CT}_{\mathrm{GT}}$ than $\mathrm{CT}_{\text {sim }}$. For the patients with the smaller prostate change over time, the procedure did not improve the dose calculations much. More research with a larger image database is necessary to find an optimal deformation metric and parameter set. With a larger database, it might be possible to find a predictive measure and criteria to decide whether DIR is worthwhile for individual patients. 


\section{ACKNOWLEDGMENTS}

The authors would like to thank D. Bouvy, prof. dr J.P.W. Pluim and in particular dr B. Reniers for their help and input on the DIR calculations.

\section{REFERENCES}

1 D. Yan, F. Vicini, J. Wong, A. Martinez, "Adaptive radiation therapy", 1997 Physics in medicine and biology (42:123-132)

2 Q.J. Wu, T. Li, Q. Wu, F.F. Yin, "Adaptive radiation therapy: technical components and clinical applications", 2011 Cancer journal (Sudbury, Mass.) (17:182-189)

3 E.K. Hansen, M.K. Bucci, J.M. Quivey, et al., "Repeat CT imaging and replanning during the course of IMRT for head-and-neck cancer", 2006 International journal of radiation oncology, biology, physics (64:355-362)

4 D.J. Fraser, Y. Chen, E. Poon, et al., "Dosimetric consequences of misalignment and realignment in prostate 3DCRT using intramodality ultrasound image guidance", 2010 Medical physics (37:2787-2795)

5 D.J. Fraser, P. Wong, K. Sultanem, F. Verhaegen, "Dosimetric evolution of the breast electron boost target using 3D ultrasound imaging", 2010 Radiotherapy and oncology : journal of the European Society for Therapeutic Radiology and Oncology (96:185-191)

6 S. van Kranen, A. Mencarelli, S. van Beek, et al., "Adaptive radiotherapy with an average anatomy model: evaluation and quantification of residual deformations in head and neck cancer patients", 2013 Radiotherapy and oncology : journal of the European Society for Therapeutic Radiology and Oncology (109:463-468)

7 Y. Yang, E. Schreibmann, T. Li, et al., "Evaluation of on-board kV cone beam CT (CBCT)-based dose calculation", 2007 Physics in medicine and biology (52:685-705)

8 S.F. Petit, W.J. van Elmpt, S.M. Nijsten, et al., "Calibration of megavoltage cone-beam CT for radiotherapy dose calculations: correction of cupping artifacts and conversion of CT numbers to electron density", 2008 Medical physics (35:849-865)

9 A. Richter, Q. Hu, D. Steglich, et al., "Investigation of the usability of conebeam CT data sets for dose calculation", 2008 Radiation oncology (3:42)

10 W. van Elmpt, S. Petit, D. De Ruysscher, et al., "3D dose delivery verification using repeated cone-beam imaging and EPID dosimetry for stereotactic body radiotherapy of non-small cell lung cancer", 2010 Radiotherapy and oncology : journal of the European Society for Therapeutic Radiology and Oncology (94:188194)

11 M. Moteabbed, G.C. Sharp, Y. Wang, et al., "Validation of a deformable image registration technique for cone beam CT-based dose verification", 2015 Medical physics (42:196)

12 C. Veiga, J. McClelland, S. Moinuddin, et al., "Toward adaptive radiotherapy for head and neck patients: Feasibility study on using CT-to-CBCT deformable registration for "dose of the day" calculations", 2014 Medical physics (41:031703)

13 J. Hatton, B. McCurdy, P.B. Greer, "Cone beam computerized tomography: the effect of calibration of the Hounsfield unit number to electron density on dose calculation accuracy for adaptive radiation therapy", 2009 Physics in medicine and biology (54:N329)

14 S. Yoo, F.-F. Yin, "Dosimetric feasibility of cone-beam CT-based treatment planning compared to CT-based treatment planning", 2006 International Journal of Radiation Oncology • Biology • Physics (66:1553-1561)

15 C. Houser, A.O. Nawaz, J. Galvin, Y. Xiao, "TH-D-ValA-04: Quantitative Evaluation of Cone Beam CT Data Used for Treatment Planning", 2006 Medical physics (33:2285-2286)

16 S. van der Meer, E. Bloemen-van Gurp, J. Hermans, et al., "Critical assessment of intramodality 3D ultrasound imaging for prostate IGRT compared to fiducial markers", 2013 Medical physics (40:071707)

17 S. van der Meer, E. Seravalli, D. Fontanarosa, et al., "Consequences of Intermodality Registration Errors for Intramodality 3D Ultrasound IGRT", 2015 Technol Cancer Res Treat)

18 S. Klein, M. Staring, K. Murphy, et al., "Elastix: A Toolbox for Intensity-Based Medical Image Registration", 2010 IEEE transactions on medical imaging (29:196-205)

19 D.P. Shamonin, E.E. Bron, B.P.F. Lelieveldt, et al., "Fast Parallel Image Registration on CPU and GPU for Diagnostic Classification of Alzheimer's Disease", 2014 Front Neuroinform (7:Article 50)

20 M. Staring, S. Klein, J.P.W. Pluim, "A rigidity penalty term for nonrigid registration", 2007 Medical physics (34:4098-4108) 
21 M.J. Ghilezan, D.A. Jaffray, J.H. Siewerdsen, et al., "Prostate gland motion assessed with cine-magnetic resonance imaging (cine-MRI)", 2005 International journal of radiation oncology, biology, physics (62:406417)

22 D.A. Low, W.B. Harms, S. Mutic, J.A. Purdy, "A technique for the quantitative evaluation of dose distributions", 1998 Medical physics (25:656-661)

23 D.A. Low, J.F. Dempsey, "Evaluation of the gamma dose distribution comparison method", 2003 Medical physics (30:2455-2464)

24 L.C.G.G. Persoon, M. Podesta, W.J.C. van Elmpt, et al., "A fast three-dimensional gamma evaluation using a GPU utilizing texture memory for on-the-fly interpolations", 2011 Medical physics (38:4032-4035)

25 M. Podesta, L.C. Persoon, F. Verhaegen, "A novel time dependent gamma evaluation function for dynamic 2D and 3D dose distributions", 2014 Physics in medicine and biology (59:5973)

26 S.J. Thomas, "Relative electron density calibration of CT scanners for radiotherapy treatment planning", 1999 Br J Radiol (72:781-786)

27 M. Fippel, "Fast Monte Carlo dose calculation for photon beams based on the VMC electron algorithm", 1999 Medical physics (26:1466-1475)

28 W.J.C. van Elmpt, S.M.J.J.G. Nijsten, R.F.H. Schiffeleers, et al., "A Monte Carlo based three-dimensional dose reconstruction method derived from portal dose images", 2006 Medical physics (33:2426-2434)

29 X. Pennec, P. Cachier, N. Ayache, "Tracking brain deformations in time sequences of 3D US images", 2003 Pattern Recognition Letters (24:801-813)

30 M. Baker, C.F. Behrens, "Prostate displacement during transabdominal ultrasound image-guided radiotherapy assessed by real-time four-dimensional transperineal monitoring", 2015 Acta Oncologica:1-7)

31 B.J. Salter, B. Wang, M.W. Szegedi, et al., "Evaluation of alignment error due to a speed artifact in stereotactic ultrasound image guidance", 2008 Physics in medicine and biology (53:N437-445)

32 D. Fontanarosa, S. van der Meer, E. Harris, F. Verhaegen, "A CT based correction method for speed of sound aberration for ultrasound based image guided radiotherapy", 2011 Medical physics (38:2665-2673)

33 D. Fontanarosa, S. van der Meer, E. Bloemen-van Gurp, et al., "Magnitude of speed of sound aberration corrections for ultrasound image guided radiotherapy for prostate and other anatomical sites", 2012 Medical physics (39:5286-5292)

34 D. Fontanarosa, S. van der Meer, F. Verhaegen, "On the significance of density-induced speed of sound variations on US-guided radiotherapy", 2012 Medical physics (39:6316-6323)

35 D. Fontanarosa, S. Pesente, F. Pascoli, et al., "A speed of sound aberration correction algorithm for curvilinear ultrasound transducers in ultrasound-based image-guided radiotherapy", 2013 Physics in medicine and biology (58:1341-1360) 


\section{SUPPLEMENTARY MATERIALS}

\section{A. Parameter file}

For the intensity based deformation

// ImageTypes

(FixedInternal ImagePixelType "float")

(MovingInternalImagePixelType "float")

(UseDirectionCosines "false")

// Components

(Registration "MultiMetricMultiResolutionRegistration")

(FixedImagePyramid "FixedRecursiveImagePyramid")

(MovingImagePyramid "MovingRecursiveImagePyramid")

(Interpolator "BSplineInterpolator")

(Optimizer "AdaptiveStochasticGradientDescent")

(ResampleInterpolator "FinalBSplineInterpola-

tor")

(Resampler "DefaultResampler")

(Transform "BSplineTransform")

// MetricType

(Metric "AdvancedNormalizedCorrelation" "TransformRigidityPenalty")

(NumberOfResolutions 3)

(HowToCombineTransforms "Compose")

(MaximumnumberOfIterations 100) (10,50 of 100)

(Metricoweight 1.0)

(Metric1Weight 10.0)

(LinearityConditionWeight 100.0)

(OrthonormalityConditionWeight 1.0)

(PropernessConditionWeight 2.0)

(UseLinearityCondition "true")

(UseorthonormalityCondition "true")

(UsePropernesscondition "true")

(CalculateLinearityCondition "true")

(CalculateorthonormalityCondition "true")

(CalculatePropernessCondition "true")

(DilateRigidityImages "false" "false" "false")

(DilationRadiusMultiplier 1.01 .01 .0 )

(UseFixedRigidityImage "false")

(UseMovingRigidityImage "true")

(MovingRigidityImageName "filepath and -name of the mask")

(FinalGridspacingInPhysicalUnits 20)

(NumberOfSpatialSamples 2500)

(NewSamplesEveryIteration "true")

( ImageSampler "Random")

(BSplineInterpolationorder 3)

(FinalBSplineInterpolationorder 1)

(DefaultPixelvalue 0)

// $\quad * * \star \star * *$ output

(WriteResultImage "true")

(ResultImagePixelType "short")

(ResultImageFormat "mhd")
Changes for the contour based deformation

(Metric "AdvancedMeanSquares" "TransformRigidityPenalty")

(MaximumnumberofIterations 300) (100 of 300) 


\section{B. Results}

Table B1: Dice coefficients (DC) of the contours of $C T_{\text {sim }}$ (Ref) and $C T_{p s}$ (parameter set A-E) compared to the ground truth. Underlined are the cases when the gamma failure increased compared to the reference situation i.e. the deformations made it worse. The DC for the contours 'Prostate US' represents the overlap of the prostate contours of US $S_{\text {sim }}$ and US tx. $_{\text {. This is, }}$ at the moment, the only measure present before deformation on which a decision can be made to perform deformations or not.

\begin{tabular}{|c|c|c|c|c|c|c|c|}
\hline \multirow[b]{2}{*}{ Patient } & \multicolumn{6}{|c|}{ Dice coefficients (Ground truth compared to ) } & \multirow[b]{2}{*}{$\mathbf{E}$} \\
\hline & Contour & Ref & A & B & C & D & \\
\hline \multirow[t]{3}{*}{1} & Prostate US & 0.43 & & & & & \\
\hline & Prostate & 0.42 & 0.71 & 0.69 & 0.68 & 0.74 & $0.75^{*}$ \\
\hline & Anus \& Rectum & 0.42 & 0.63 & 0.69 & $0.70^{*}$ & 0.62 & 0.66 \\
\hline \multirow[t]{4}{*}{2} & Prostate US & 0.70 & & & & & \\
\hline & Prostate & 0.50 & $\underline{0.47}$ & $\underline{0.49}$ & 0.50 & $0.60 *$ & - \\
\hline & Anus \& Rectum & 0.66 & $\underline{0.62}$ & 0.70 & 0.71 & $0.74 *$ & - \\
\hline & Bladder & 0.63 & $\underline{0.62}$ & $\underline{0.63}$ & $\underline{0.63}$ & $0.68 *$ & - \\
\hline \multirow[t]{4}{*}{$3 a$} & Prostate US & 0.50 & & & & & \\
\hline & Prostate & 0.82 & $\underline{0.59}$ & $\underline{0.44}$ & $\underline{0.41}$ & $\underline{0.62}$ & $\underline{0.62}$ \\
\hline & Anus \& Rectum & 0.66 & $\underline{0.43}$ & $\underline{0.24}$ & $\underline{0.20}$ & $\underline{0.45}$ & $\underline{0.45}$ \\
\hline & Bladder & 0.84 & $\underline{0.76}$ & $\underline{0.76}$ & $\underline{0.75}$ & $\underline{0.75}$ & $\underline{0.76}$ \\
\hline \multirow[t]{4}{*}{$3 b$} & Prostate US & 0.54 & & & & & \\
\hline & Prostate & 0.75 & $\underline{0.61}$ & $\underline{0.63}$ & $\underline{0.62}$ & $0.77^{*}$ & 0.75 \\
\hline & Anus \& Rectum & 0.59 & $\underline{0.57}$ & $\underline{0.59}$ & $\underline{0.59}$ & $0.69 *$ & 0.66 \\
\hline & Bladder & 0.77 & 0.79 & 0.79 & 0.80 & 0.83 & $0.83 *$ \\
\hline \multirow[t]{4}{*}{$3 c$} & Prostate US & 0.42 & & & & & \\
\hline & Prostate & 0.55 & $\underline{0.51}$ & $\underline{0.52}$ & $\underline{0.47}$ & 0.73 & $0.75^{*}$ \\
\hline & Anus \& Rectum & 0.46 & 0.49 & 0.56 & 0.62 & $0.64 *$ & 0.63 \\
\hline & Bladder & 0.73 & 0.73 & $\underline{0.64}$ & $\underline{0.63}$ & 0.82 & $0.82 *$ \\
\hline \multirow[t]{4}{*}{$3 d$} & Prostate US & 0.28 & & & & & \\
\hline & Prostate & 0.40 & 0.45 & 0.60 & 0.67 & 0.76 & $0.78 *$ \\
\hline & Anus \& Rectum & 0.29 & 0.32 & 0.40 & 0.44 & $0.55^{*}$ & 0.55 \\
\hline & Bladder & 0.68 & 0.71 & 0.76 & $0.78^{*}$ & 0.76 & 0.76 \\
\hline
\end{tabular}

* Parameter set with the best improvement.

Abbreviations: $\mathrm{GT}$, ground truth; Ref, reference situation of $\mathrm{CT}_{\text {sim }}$ compared to $\mathrm{CT}_{\mathrm{GT}}$; 
Table B2: Gamma evaluation on the CT values where the Hounsfield units (HU) on the $C T_{\text {sim }}$ (Ref) and $C T_{p s}$ images (metric $\left.A-E\right)$ were compared to the $H U$ on $C T_{G T}$. Shown is the volume percentage of a gamma failure $\left(\left|\gamma_{\mathrm{CT}(5 \mathrm{OHU}, 3 \mathrm{~mm})}\right|>1\right)$ of the different contours. Underlined are the cases when the gamma failure increased compared to the reference situation i.e. the deformations made it worse.

\begin{tabular}{|c|c|c|c|c|c|c|c|}
\hline \multirow[b]{2}{*}{ Patient } & \multirow[b]{2}{*}{ Contour } & \multicolumn{5}{|c|}{ Volume $|\gamma \mathrm{CT}(50 \mathrm{HU}, 3 \mathrm{~mm})|>1$ (\%) } & \multirow[b]{2}{*}{$\mathbf{E}$} \\
\hline & & Ref & A & B & C & D & \\
\hline \multirow[t]{3}{*}{1} & Body $(\cap)$ & 14.3 & 9.9 & 11.5 & 12.7 & $9.7^{*}$ & 11.2 \\
\hline & Prostate & 12.0 & 4.6 & $2.9^{*}$ & 3.0 & 5.3 & 7.1 \\
\hline & Anus \& Rectum & 22.0 & 14.5 & $13.4^{*}$ & 13.6 & 15.2 & 16.1 \\
\hline \multirow[t]{4}{*}{2} & Body (n) & 23.1 & 21.7 & $19.8^{*}$ & 19.9 & 19.9 & - \\
\hline & Prostate & 11.5 & $\underline{14.6}$ & $\underline{12.8}$ & $\underline{12.3}$ & $10.2^{*}$ & - \\
\hline & Anus \& Rectum & 28.9 & $25.4^{*}$ & 27.3 & 28.8 & 28.1 & - \\
\hline & Bladder & 10.4 & 7.7 & 4.7 & $3.7^{*}$ & 5.8 & - \\
\hline \multirow[t]{4}{*}{$3 a$} & Body $(\cap)$ & 8.5 & 7.4 & $6.8^{*}$ & 7.2 & 8.0 & 8.0 \\
\hline & Prostate & 6.9 & $\underline{7.4}$ & $\underline{7.8}$ & $\underline{8.5}$ & $\underline{7.5}$ & $\underline{7.3}$ \\
\hline & Anus \& Rectum & 22.7 & $20.3^{*}$ & 22.5 & $\underline{23.9}$ & 21.1 & 21.5 \\
\hline & Bladder & 4.2 & 3.0 & $2.7^{*}$ & 3.2 & $\underline{6.5}$ & $\underline{6.4}$ \\
\hline \multirow[t]{4}{*}{$3 b$} & Body $(\cap)$ & 10.6 & 9.8 & 8.2 & 7.9 & $5.7^{*}$ & 6.0 \\
\hline & Prostate & 6.6 & $\underline{6.6}$ & $\underline{7.0}$ & $\underline{6.8}$ & $5.0^{*}$ & 5.1 \\
\hline & Anus \& Rectum & 11.7 & 7.0 & $6.7^{*}$ & 7.2 & 11.2 & $\underline{12.9}$ \\
\hline & Bladder & 11.1 & 8.0 & 7.0 & $6.6^{*}$ & 7.4 & 7.7 \\
\hline \multirow[t]{4}{*}{$3 c$} & Body $(\cap)$ & 18.1 & 16.5 & 15.0 & 13.5 & 12.8 & $12.7^{*}$ \\
\hline & Prostate & 9.9 & 7.1 & 7.7 & 7.2 & 7.9 & $6.8^{*}$ \\
\hline & Anus \& Rectum & 19.0 & 14.2 & 11.5 & $9.3^{*}$ & 12.9 & 12.4 \\
\hline & Bladder & 7.6 & 5.9 & 6.6 & 6.5 & $1.2^{*}$ & 1.5 \\
\hline \multirow[t]{4}{*}{$3 d$} & Body $(\cap)$ & 23.8 & 21.9 & 19.7 & 18.5 & 18.3 & $17.8^{*}$ \\
\hline & Prostate & 11.8 & 6.7 & 4.4 & $4.0^{*}$ & 10.1 & 9.3 \\
\hline & Anus \& Rectum & 32.8 & 24.2 & 14.9 & $12.3^{*}$ & 18.3 & 18.2 \\
\hline & Bladder & 20.8 & 17.1 & 12.2 & $9.6^{*}$ & 11.5 & 11.6 \\
\hline
\end{tabular}

* Parameter set with the best improvement.

Abbreviations: $\gamma_{C T}$, gamma evaluated on the $C T$ values; $n$, intersection of the body contours of $\mathrm{CT}_{\text {sim }}$ and $\mathrm{CT}_{\mathrm{GT}} ; \mathrm{GT}$, ground truth; $\mathrm{HU}$, Hounsfield units; Ref, reference situation of $\mathrm{CT}_{\text {sim }}$ compared to $\mathrm{CT}_{\mathrm{GT}}$; 
(a) Dice coefficient

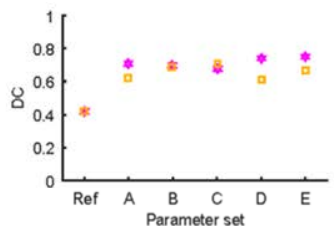

(d) Dice coefficient

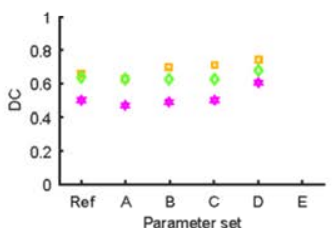

(g) Dice coefficient

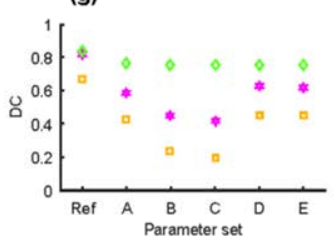

(j) Dice coefficient

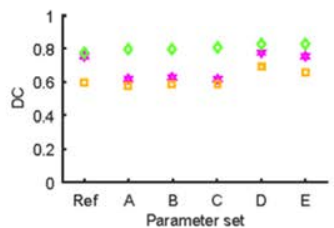

(m) Dice coefficient

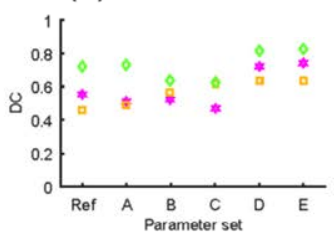

(p) Dice coefficient

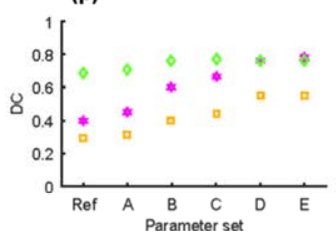

(b) Gamma on CT values

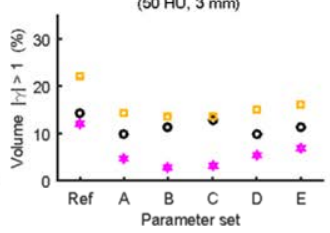

(e) Gamma on CT values

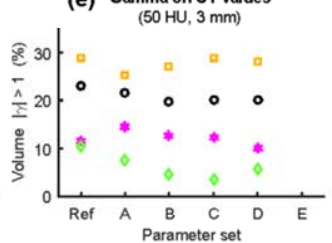

(h) Gamma on CT values

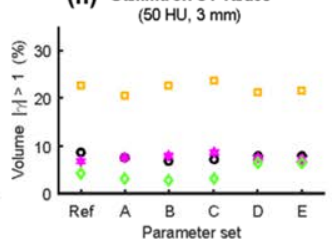

(k) Gamma on CT values

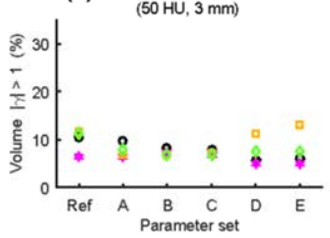

(n) Gamma on CT values

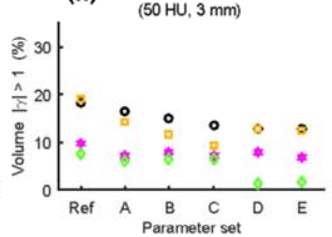

(q) Gamma on CT values

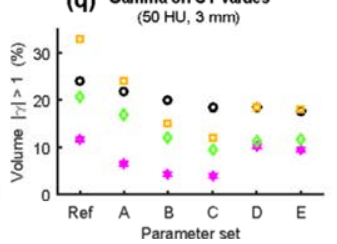

(c) Gamma on dose

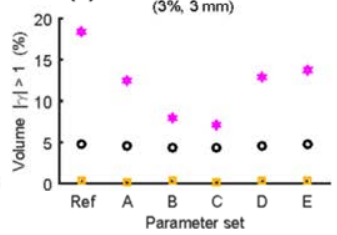

Patient 1

․ㅗㅁ Pody

Prostase

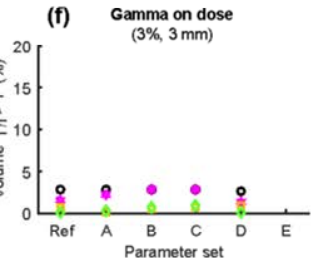

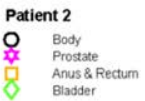

Patient 3a

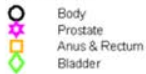

Patient 3b
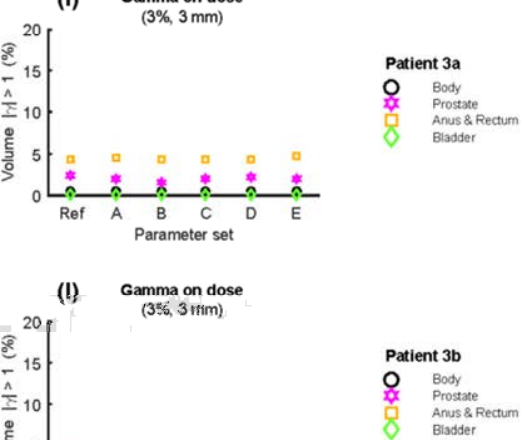

(I) Gamma on dose

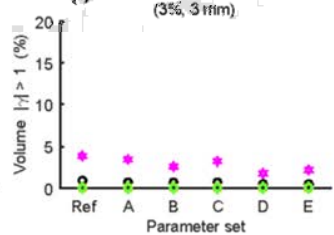

(o) Gamma on dose

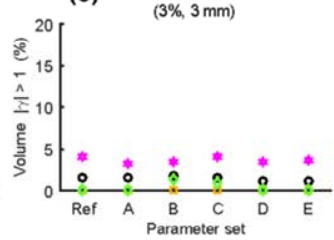

Patient 3c
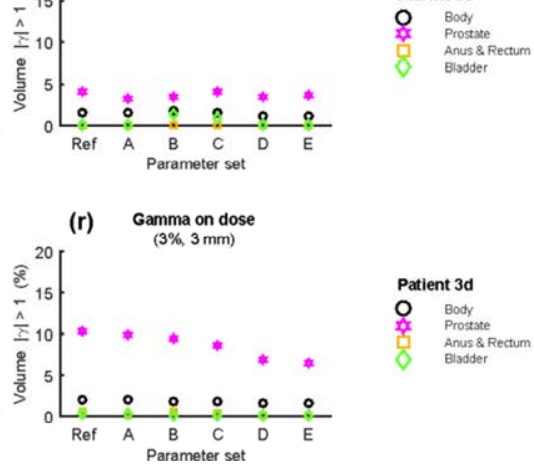

Patient 3d

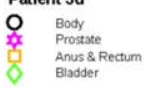

Figure B: Results for all five different parameter sets. In the first column, the results of the dice coefficients is plotted. In the second column, the gamma evaluation on the CT values is shown. The percentage of a gamma failure $\left(\left|\gamma_{\mathrm{CT}(5 \mathrm{OHU}, 3 \mathrm{~mm})}\right|>1\right)$ is plotted. The last column shows the gamma evaluation on the dose. Again the percentage of a gamma failure $\left(\left|\nu_{\text {Dose }(3 \%, 3 \mathrm{~mm})}\right|>1\right)$ is plotted. 


\section{DISCUSSION}





\section{CHAPTER}

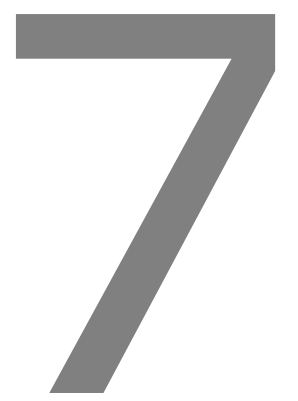

Summary, general discussion and future perspectives 



\section{SUMMARY AND GENERAL DISCUSSION}

\section{Image-guided radiotherapy}

Since the introduction of intensity-modulated radiotherapy (IMRT $)^{1,2}$, and now with the rotational techniques volumetric modulated arc therapy (VMAT) $)^{3,4}$, dose distributions can be created that have complex concave shapes and steep dose gradients resulting in a better sparing of organs at risk, which was not possible with 3D conformal radiotherapy.

In order to exploit the full potential of these techniques, it is crucial that patient positioning is more accurate and reproducible than it is for 3D conformal radiotherapy. Imageguided radiotherapy (IGRT) plays this role and has in many clinics been introduced almost together with IMRT. With all IGRT techniques, the actual anatomy is imaged and aligned with the anatomy that was used during the design of the treatment plan, i.e. usually the planning CT scan. With IGRT, high-precision patient positioning is available and necessary to make optimal use of the possible advantage of IMRT and VMAT.

Various IGRT methods have been investigated to localize the target during radiotherapy treatment. In general, one could differentiate systems by their use of ionizing radiation or not, or by their soft-tissue contrast. The non-ionizing radiation techniques, US and $\mathrm{MRI}$, have in most cases high soft tissue contrast. In $\mathrm{CT}$ and $\mathrm{CBCT}^{5,6}$ the soft tissue contrast is moderate, and in portal imaging ${ }^{7}$ only objects with high densities such as bones are easily visible. To compensate for lack of contrast, implanted fiducial markers (FMs) ${ }^{8}$ with a high density can be used as a surrogate to localize the position of the soft tissue organs.

From a practical point of view, CBCT and portal imagers have the benefit of being fully integrated in most linear accelerators. The integration of MRI and a linear accelerators is under development ${ }^{9}$, but in-room solutions for both $\mathrm{MRI}^{10,11}$ and $\mathrm{CT}^{12}$ are already clinically available. This, however, requires large treatment rooms. US imaging systems are less space consuming and less costly, but require patient contact and a user or robot-arm in the treatment room.

\section{US image-guided radiotherapy}

\section{Inter-fraction motion monitoring}

Even though US IGRT has been around for several years, only lately there is a new growing interest in its developments. Since 2004 the Clarity 3DUS guidance system, a 3D intramodality system, was introduced. The intramodality approach entails comparison of an US image made treatment stage, with a reference US image, acquired at the time of CT simulation, and therefore compares like with like. In this thesis we focused on the implementation of this 3D intramodality US IGRT technique in the clinic for prostate and liver scanning, and looked at the different aspects that had to be investigated on forehand.

Primarily, the US system itself has to be accurate ${ }^{13,14}$, and the system has to be fully understood to avoid mistakes and misusage. In Chapter 3, we investigated the workflow and looked at consequences of possible errors on the intermodality procedures of the system. We found that The Clarity 3DUS guidance system is a robust IGRT device that 
guides the patient to the correct treatment position under the following conditions: the metal markers are used for the registration of the CT; Speed of sound (SOS) aberrations are taken into account ${ }^{15,16}$ in intermodality image fusion, and preferably also during the intramodality procedures (both US images); and the CT scan and (SOS corrected) reference US scan match. In the case of a mismatch, it is advised to rescan the patient with both CT and reference US in direct succession with good patient stabilization to prevent patient motion in between the scans.

For the mentioned SOS artifacts, our group has developed a correction algorithm to account for these aberrations ${ }^{15,16}$. The algorithm was tested with phantom measurements, as well as with clinical examples in breast, prostate and liver and was found to be able to provide an improvement in the special accuracy of US imaging ${ }^{15-18}$.

Further, one of the major concerns for US IGRT is the user variability. Not only is the acquisition of the image for most US systems still performed manually, the images may also be more difficult to interpret than e.g. a CT or MR image. The complete body contour is not visible on an US image, therefore structures may be more difficult to identify on an US image. User experience and training have been shown to improve the consistency and reproducibility of US image interpretation among users ${ }^{19,20}$. In most US systems there are several time points at which the user variability can have an influence; during image acquisition, structure contouring and at both the inter- and intramodality matching stages. For prostate (Chapter 4 ) and liver (Chapter 5) some of the variabilities have been investigated and can therefore be taken into account in the uncertainty of the whole procedure.

One source of large user variability during image acquisition may be probe pressure. Several studies ${ }^{21,22}$ have shown a potential influence of probe pressure on the prostate position and we also shown our own results in Chapter 4 . The prostate displacement increases when more pressure is applied, yet no obvious trend in prostate displacement was observed in our patient group. The average displacement vector was about $3 \mathrm{~mm}$ for a $1 \mathrm{~cm}$ skin displacement which would occur in normal clinical practice. For intramodality systems, in theory the probe pressure distortion is minimized when similar scanning procedures are used to acquire the images during simulation and treatment stages; the corrective shifts are derived as differences between two images, both affected by (ideally) the same probe pressure. Probe pressure can be avoided completely by scanning the prostate transperineally, e.g. by the Clarity Autoscan system ${ }^{23}$. Another solution to achieve highly reproducible probe pressure could be by using robot-arms ${ }^{24-27}$.

To define the accuracy of the US IGRT procedure, preferably the position measured by the US system has to be compared to the 'true' position of the target. Unfortunately, the 'true' position of an organ is difficult to determine, and none of the available localization techniques are true gold standards. Most studies compare the localization from US to another IGRT technique, where usually the reference technique is already clinically implemented (Chapter 2, table 6). Some studies reported clinically significant differences. This should not immediately lead to the conclusion that the US IGRT system is incorrect. Nonetheless, with clinically significant differences between systems, changing from one system to another can result in a better or worse patient treatment.

In Chapter 4, we compared the US IGRT workflow with our clinical practice at that time: prostate localization based on implanted FMs with portal imaging. The overall accuracy (including user variability) of US $(3.0 \mathrm{~mm}$ ) is comparable to our FM workflow (2.2 
$\mathrm{mm}$ ). Since neither US nor FM can be considered a gold standard no conclusions can be drawn on the superiority of either method. It requires more effort to standardize US imaging than FM detection, but because US imaging captures the prostate itself instead of surrogates no invasive procedure is required. Besides, since US imaging does not involve a radiation burden, US prostate imaging can offer an interesting alternative for positioning with FMs in combination with portal imaging. In this thesis, we did not compare the US IGRT system with CBCT, but other studies did, and their conclusions are comparable ${ }^{28-30}$.

In Chapter $\mathbf{5}$ we investigated the use of US IGRT application in liver treatments. The feasibility of US imaging guidance for liver was already demonstrated by Boda-Heggemann et al. $^{31}$, using the 2D US BAT device (Nomos, Cranberry Township, PA). This allows for superposition of anatomic contours derived from treatment planning CT onto real-time US images, for liver image guidance. The use of two different image modalities in an intermodality system, however, introduces additional uncertainty. In our study, we showed that also intramodality 3D US imaging for IGRT of liver lesions was found to be feasible, although using anatomic surrogates in the close vicinity of the lesion may be needed. The accuracy based on the user variability could be improved by using active breathing control; in free breathing the accuracy was $4 \mathrm{~mm}$, and when it is combined with active breathing control the accuracy was $2 \mathrm{~mm}$. For liver US IGRT, a one-on-one comparison of the US shifts with bony anatomy based portal imaging IGRT (our clinical practice at that time) was given in a short report in the appendix of Chapter 5 . The results also show that US IGRT would be a good localization method for liver SBRT.

Chapter 4 and 5 are together with many other studies on inter-fraction US IGRT reviewed in Chapter 2.

The objective of this thesis was to determine the accuracy of a 3D US imaging technique for image guidance, to determine the daily position and volume of prostate and liver lesions and analyze whether the accuracy of 3D US is compatible with the accuracy of EPI for image guidance. From Chapter 3, 4 and 5 we learned that the accuracy of US imaging is comparable to EPI image guidance as long as you follow the correct procedures. The fact that US and EPI IGRT might not be directly interchangeable without a clinical effect is mainly due to the fact that EPI visualizes the bones or FM and US looks at soft tissue targets. In particular for liver we found that US IGRT is preferred over EPI image guidance. The feasibility for prostate is shown as well, in particular a better prostate volume representation, only a minimally increased treatment time, avoidance of FM implantation (removing the risk of bleeding and prostate inflammation), and avoidance of extra radiation burden shows that prostate localization with US imaging is a reasonable alternative for patients for whom FM MV EPI positioning is not desirable. Overall, The US IGRT was found to be a non-invasive and accurate technique for image guidance with the feasibility to correct for inter-fraction organ motion.

\section{Intra-fraction motion monitoring}

Not part of this thesis, but already commercially available for the prostate is the Clarity Autoscan $^{23}$, which is capable of intra-fraction motion tracking i.e. motion tracking during the course of treatment. US has a high temporal resolution and is therefore a true real- 
time imaging technique that can track tissue deformation by direct monitoring of internal tissue motion. In combination with the zero dose and non-invasiveness, it is an ideal candidate for intra-fraction motion tracking. With the Clarity Autoscan, the transducer is positioned for transperineal prostate imaging. During treatment, 3D US images are acquired at $2.5 \mathrm{~s}$ intervals and registered to a reference US volume using a correlation-based search with reference ROls centered on pixels within $2 \mathrm{~cm}$ of the prostate boundary. Phantom studies demonstrate that $<1.2 \mathrm{~mm}$ accuracy and precision of motion estimation can be achieved using Clarity Autoscan ${ }^{23,32}$.

A handful of studies have shown the feasibility of 2D US-based motion tracking of the diaphragm $^{33}$, liver ${ }^{34}$, prostate p $^{24,25}$ and lung surface ${ }^{34}$. Liver motion tracking in 3D was demonstrated by Harris et $a .^{35}$. A full review on this topic by $\mathrm{O}^{\prime}$ Shea et $a l^{36}{ }^{36}$ is currently under review for publication in the journal 'Physics in medicine and biology'.

\section{Current clinical use of US IGRT}

At the moment there are two commercially available US IGRT systems for inter-fraction motion correction: BAT (Best Nomos, Pittsburgh, PA, USA) and Clarity (Elekta, Stockholm, Sweden). The main clinical usage of US IGRT is during prostate and breast radiotherapy. But in some research institutes also other target sites are treated with US guidance. A 3DUS system was designed for extracranial stereotactic guidance (Meeks et al 2003), which later led to the commercial SonArray system (ZMed, Inc, Ashland, MA, USA; later this system was acquired by Varian Medical Systems, Palo Alto, CA, USA), but it is now no longer marketed. Already in 2004 the utility of the BAT system was investigated for 62 patients with upper abdominal tumors ${ }^{37}$ and it was successfully implemented in the clinical routine. In 2006, Boda-Heggemann et al. ${ }^{31}$ first reported on only liver but continued on the rest of the upper abdomen ${ }^{38}$ and proved the feasibility in that region as well. Other groups reported on gallbladder ${ }^{39}$, pancreas $^{40}$ and uterus ${ }^{41}$. In the review of Chapter $\mathbf{2}$, an overview is given of all target sites that were under investigation for the use of US IGRT.

For intra-fraction motion tracking only one system, Clarity Autoscan (Elekta, Stockholm, Sweden) is commercially available and is currently only used for prostate tracking ${ }^{23 \text {, }}$ 32,42

\section{FUTURE PERSPECTIVES}

Currently, it is not easy to predict the future of US imaging in RT. However, until now widespread US use in RT has been hampered due to the extensive training requirements. Therefore there is an urgent need to make US imaging user-independent and automatic. Some recent advances have been reported in this direction by using robotic operator less systems ${ }^{23-27}$. Much work is also being done to segment structures automatically in real time, and one can envisage applications such as real-time dose display based on anatomical 3D deformation fields derived from daily US imaging. These new developments, in 
combination with automated operator-less imaging may lead to a much higher clinical acceptance of the US IGRT technique in RT.

Also the US image quality has improved significantly recently and besides interfraction motion correction, intra-fraction motion tracking will become more and more important. Therefore US has the capabilities of becoming an important player in that field, not only for prostate, but for other tumor sites as well. Research like discussed in the last part of the previous section will be one of the focus points currently and in the future.

But not only intra-fraction motion is a field of interest for US guidance in radiotherapy. In principle, also with US Imaging we can go beyond geometrical treatment correction and enter the field of adaptive radiotherapy (ART) ${ }^{43,44}$ (Chapter 6).

\section{Adaptive radiotherapy}

Image information acquired with IGRT during treatment is currently used for patient positioning and alignment. The next step is to use this information as a starting point for adjustments or adaptation of the treatment. This procedure is often referred to as $A R T^{43 \text {, }}$ ${ }^{44}$. The basis of this type of image-guided ART is the frequent (on-line) imaging possibilities nowadays available in a radiotherapy department and adaptation of the treatment plan based on the changes observed in the images. Normally one would need (CB)CT for this but in Chapter 6 we showed that it was possible to generate a pseudo-CT with the use of deformable image registration (DIR) based on US imaging which was more representative of the ground truth $\mathrm{CT}$ than the plannings $\mathrm{CT}$. For the patients with the smaller prostate change over time, the procedure did not improve the dose calculations much and more research with a larger image database is necessary to find an optimal deformation metric and parameter set. With a larger database, it might be possible to find a predictive measure and criteria to decide whether DIR is worthwhile for individual patients.

\section{Functional and molecular information}

Besides during treatment, more steps in the radiotherapy workflow can gain from US imaging (Chapter 2, figure 1). In the review of O'Shea et al. ${ }^{36}$ selections of existing and proposed novel applications of US imaging are discussed which can provide functional and molecular information that can be used for target definition or treatment response assessment of the radiotherapy treatment. These applications are driven by exciting developments in structural, functional and molecular US imaging and analytical techniques such as backscatter tissue analysis, elastography, photo-acoustography, Doppler imaging, contrast-specific imaging, dynamic contrast analysis, microvascular and super-resolution imaging, and targeted microbubbles and nanoparticles. These techniques show promise for predicting and measuring the progression of radiotherapy treatment, quantifying normal tissue toxicity, improving tumor definition and defining a biological target volume that describes radiation sensitive regions of the tumor. Ultrasound contrast technology also has potential to be used actively to assist RT by manipulating the tumor cell environment and by improving the delivery of radiosensitising agents. 


\section{Final remarks}

The current applications of IGRT in particle beam therapy are still limited, but with an increasing number of particle beam indications and facilities, the need to integrate image guidance in this field will become more and more important. Integration of US imaging in the particle beam systems could result in new opportunities that also facilitate real-time organ monitoring. In particular with new developed US transducers that can stick to the skin increase the usability for US IGRT. Still, challenges such as avoidance of the US transducer with scanning particle beams will have to be addressed. Additionally, renewed investigations of already old US studies revealed new opportunities for real-time in vivo particle beam range monitoring. The acoustic signal that is generated due to the localized ion energy deposition in an absorber is detected ${ }^{45-49}$. With the use of tomographic reconstruction, not only the ion range can be measured, also the beam spot size at the Bragg peak position can be determined ${ }^{50,51}$.

Lastly, not only with US there are currently new developments ongoing. Already a few years people are working on the integration of MRI with a linear accelerator ${ }^{9}$ and the expectations are high. Also here the MRI offers high imaging contrast during therapy, but the costs are high. MRI, US and other imaging modalities are to some extent complementary. Therefore, future radiotherapy departments should have access to multiple forms of imaging for treatment guidance that could be used in multiple combinations, depending on the application.

\section{REFERENCES}

1 G. Intensity Modulated Radiation Therapy Collaborative Working, "Intensity-modulated radiotherapy: current status and issues of interest", 2001 International journal of radiation oncology, biology, physics (51:880-914)

2 G.A. Ezzell, J.M. Galvin, D. Low, et al., "Guidance document on delivery, treatment planning, and clinical implementation of IMRT: report of the IMRT Subcommittee of the AAPM Radiation Therapy Committee", 2003 Medical physics (30:2089-2115)

3 K. Otto, "Volumetric modulated arc therapy: IMRT in a single gantry arc", 2008 Medical physics (35:310317)

4 M. Teoh, C.H. Clark, K. Wood, et al., "Volumetric modulated arc therapy: a review of current literature and clinical use in practice", 2011 Br J Radiol (84:967-996)

5 M. Oldham, D. Letourneau, L. Watt, et al., "Cone-beam-CT guided radiation therapy: A model for on-line application", 2005 Radiotherapy and oncology : journal of the European Society for Therapeutic Radiology and Oncology (75:271-278)

6 G.G. Zeng, T.S. McGowan, T.M. Larsen, et al., "Calcifications are potential surrogates for prostate localization in image-guided radiotherapy", 2008 International journal of radiation oncology, biology, physics (72:963-966)

7 L.M. Girouard, J. Pouliot, X. Maldague, A. Zaccarin, "Automatic setup deviation measurements with electronic portal images for pelvic fields", 1998 Medical physics (25:1180-1185)

8 U.A. van der Heide, A.N. Kotte, H. Dehnad, et al., "Analysis of fiducial marker-based position verification in the external beam radiotherapy of patients with prostate cancer", 2007 Radiotherapy and oncology : journal of the European Society for Therapeutic Radiology and Oncology (82:38-45)

9 J.J. Lagendijk, B.W. Raaymakers, A.J. Raaijmakers, et al., "MRI/linac integration", 2008 Radiotherapy and oncology : journal of the European Society for Therapeutic Radiology and Oncology (86:25-29)

10 Y. Hu, O.L. Green, Y. Feng, et al., "Image Performance Characterization of an MRI-Guided Radiation Therapy System", 2013 International journal of radiation oncology, biology, physics (87:S13)

11 D.A. Jaffray, M. Carlone, S. Breen, et al., "Development of a Novel Platform for MR-Guided Radiation Therapy", 2013 International journal of radiation oncology, biology, physics (87:S13) 
12 K. Knight, N. Touma, L. Zhu, et al., "Implementation of daily image-guided radiation therapy using an inroom CT scanner for prostate cancer isocentre localization", 2009 Journal of Medical Imaging and Radiation Oncology (53:132-138)

$13 \mathrm{H}$. Ballhausen, S. Hieber, M. Li, et al., "Technical Note: Millimeter precision in ultrasound based patient positioning: experimental quantification of inherent technical limitations", 2014 Medical physics (41:081718)

14 H. Ballhausen, B.D. Ballhausen, M. Lachaine, et al., "Surface refraction of sound waves affects calibration of three-dimensional ultrasound", 2015 Radiat Oncol (10:119)

15 D. Fontanarosa, S. van der Meer, E. Harris, F. Verhaegen, "A CT based correction method for speed of sound aberration for ultrasound based image guided radiotherapy", 2011 Medical physics (38:2665-2673)

16 D. Fontanarosa, S. Pesente, F. Pascoli, et al., "A speed of sound aberration correction algorithm for curvilinear ultrasound transducers in ultrasound-based image-guided radiotherapy", 2013 Physics in medicine and biology (58:1341-1360)

17 D. Fontanarosa, S. van der Meer, E. Bloemen-van Gurp, et al., "Magnitude of speed of sound aberration corrections for ultrasound image guided radiotherapy for prostate and other anatomical sites", 2012 Medical physics (39:5286-5292)

18 D. Fontanarosa, S. van der Meer, F. Verhaegen, "On the significance of density-induced speed of sound variations on US-guided radiotherapy", 2012 Medical physics (39:6316-6323)

19 M. Fuss, S.X. Cavanaugh, C. Fuss, et al., "Daily stereotactic ultrasound prostate targeting: inter-user variability", 2003 Technology in cancer research \& treatment (2:161-170)

20 C. Fiandra, A. Guarneri, F. Munoz, et al., "Impact of the observers' experience on daily prostate localization accuracy in ultrasound-based IGRT with the Clarity platform", 2014 Journal of applied clinical medical physics / American College of Medical Physics (15:4795)

21 C.F. Serago, S.J. Chungbin, S.J. Buskirk, et al., "Initial experience with ultrasound localization for positioning prostate cancer patients for external beam radiotherapy", 2002 International journal of radiation oncology, biology, physics (53:1130-1138)

22 X. Artignan, M.H. Smitsmans, J.V. Lebesque, et al., "Online ultrasound image guidance for radiotherapy of prostate cancer: impact of image acquisition on prostate displacement", 2004 International journal of radiation oncology, biology, physics (59:595-601)

23 M. Lachaine, T. Falco, "Intrafractional prostate motion management with the Clarity autoscan system", 2013 Medical Physics International (1:72-80)

24 J. Schlosser, K. Salisbury, D. Hristov, "Telerobotic system concept for real-time soft-tissue imaging during radiotherapy beam delivery", 2010 Medical physics (37:6357-6367)

25 J. Schlosser, K. Salisbury, D. Hristov, "Online image-based monitoring of soft-tissue displacements for radiation therapy of the prostate", 2012 International journal of radiation oncology, biology, physics (83:1633-1640)

26 J. Schlosser, C. Kirmizibayrak, V. Shamdasani, et al., "Automatic 3D ultrasound calibration for image guided therapy using intramodality image registration", 2013 Physics in medicine and biology (58:7481-7496)

27 M.A. Lediju Bell, H.T. Sen, I. Iordachita, et al., presented at the Proc. SPIE 9036, Medical Imaging 2014: Image-Guided Procedures, Robotic Interventions, and Modeling, San Diego, California, USA, 2014 (unpublished).

28 E. Mayyas, I.J. Chetty, M. Chetvertkov, et al., "Evaluation of multiple image-based modalities for imageguided radiation therapy (IGRT) of prostate carcinoma: A prospective study", 2013 Medical physics (40:041707)

29 H. Ballhausen, S. Hieber, M. Li, et al., "Linearity of patient positioning detection : a phantom study of skin markers, cone beam computed tomography, and 3D ultrasound", 2015 Strahlentherapie und Onkologie : Organ der Deutschen Rontgengesellschaft ... [et al] (191:442-447)

30 M. Li, H. Ballhausen, N.S. Hegemann, et al., "A comparative assessment of prostate positioning guided by three-dimensional ultrasound and cone beam CT", 2015 Radiat Oncol (10:82)

31 J. Boda-Heggemann, C. Walter, S. Mai, et al., "Frameless stereotactic radiosurgery of a solitary liver metastasis using active breathing control and stereotactic ultrasound", 2006 Strahlentherapie und Onkologie : Organ der Deutschen Rontgengesellschaft ... [et al] (182:216-221)

32 M.C. Abramowitz, E. Bossart, R. Flook, et al., "Noninvasive Real-time Prostate Tracking Using a Transperineal Ultrasound Approach", 2012 International journal of radiation oncology, biology, physics (84:S133) 
33 Q. Xu, R.J. Hamilton, "A novel respiratory detection method based on automated analysis of ultrasound diaphragm video", 2006 Medical physics (33:916-921)

34 J.M. Rubin, M. Feng, S.W. Hadley, et al., "Potential use of ultrasound speckle tracking for motion management during radiotherapy: preliminary report", $2012 \mathrm{~J}$ Ultrasound Med (31:469-481)

35 E.J. Harris, N.R. Miller, J.C. Bamber, et al., "Speckle tracking in a phantom and feature-based tracking in liver in the presence of respiratory motion using 4D ultrasound", 2010 Physics in medicine and biology (55:33633380)

36 T. O'Shea, J. Bamber, D. Fontanarosa, et al., "Review of Ultrasound Image Guidance in External Beam Radiotherapy, Part 2: intra-fraction motion management and novel applications.", 2015 Phys Med Biol (submitted))

37 M. Fuss, B.J. Salter, S.X. Cavanaugh, et al., "Daily ultrasound-based image-guided targeting for radiotherapy of upper abdominal malignancies", 2004 International journal of radiation oncology, biology, physics (59:1245-1256)

38 J. Boda-Heggemann, P. Mennemeyer, H. Wertz, et al., "Accuracy of ultrasound-based image guidance for daily positioning of the upper abdomen: an online comparison with cone beam CT", 2009 International journal of radiation oncology, biology, physics (74:892-897)

39 C.D. Fuller, C.R. Thomas, Jr., A. Wong, et al., "Image-guided intensity-modulated radiation therapy for gallbladder carcinoma", 2006 Radiotherapy and oncology : journal of the European Society for Therapeutic Radiology and Oncology (81:65-72)

40 M. Fuss, A. Wong, C.D. Fuller, et al., "Image-guided intensity-modulated radiotherapy for pancreatic carcinoma", 2007 Gastrointestinal cancer research : GCR (1:2-11)

41 M. Baker, J.A. Jensen, C.F. Behrens, presented at the Proc. IEEE Ultrasonics Symposium, Prague, 2013 (unpublished).

42 M. Fargier-Voiron, L. Guillet, P. Pommier, et al., "MO-DE-210-04: Repositioning and Monitoring of Prostate Cancer Radiotherapy with a New 4D Ultrasound Intra-Modality IGRT Device", 2015 Medical physics $(42: 3560)$

43 D. Yan, F. Vicini, J. Wong, A. Martinez, "Adaptive radiation therapy", 1997 Physics in medicine and biology (42:123-132)

44 Q.J. Wu, T. Li, Q. Wu, F.F. Yin, "Adaptive radiation therapy: technical components and clinical applications", 2011 Cancer journal (17:182-189)

45 M. Levi, T. Armstrong, H. Baranger, et al., "Experimental studies of the acoustic signature of proton beams traversing fluid media", 1978 Journal Name: IEEE Trans. Nucl. Sci.; (United States); Journal Volume: NS25:1:Medium: X; Size: Pages: 325-332)

46 L. Sulak, T. Armstrong, H. Baranger, et al., "Experimental studies of the acoustic signature of proton beams traversing fluid media", 1979 Nuclear Instruments and Methods (161:203-217)

47 Y. Hayakawa, J. Tada, N. Arai, et al., "Acoustic pulse generated in a patient during treatment by pulsed proton radiation beam", 1995 Radiation Oncology Investigations (3:42-45)

48 V.I. Albul, V.B. Bychkov, S.S. Vasil'ev, et al., "Acoustic field generated by a beam of protons stopping in a water medium", 2005 Acoust. Phys. (51:33-37)

49 T. Terunuma, T. Sakae, Y. Hayakawa, et al., "Waveform simulation based on 3D dose distribution for acoustic wave generated by proton beam irradiation", 2007 Medical physics (34:3642-3648)

50 L. Xiang, B. Han, C. Carpenter, et al., "X-ray acoustic computed tomography with pulsed x-ray beam from a medical linear accelerator", 2013 Medical physics (40:010701)

51 W. Assmann, S. Kellnberger, S. Reinhardt, et al., "lonoacoustic characterization of the proton Bragg peak with submillimeter accuracy", 2015 Medical physics (42:567-574) 
SOCIETAL IMPACT AND VALORIZATION 



\section{SOCIETAL IMPACT AND VALORIZATION}

Because the topic of this thesis is already very clinically oriented, most of the valorisation is already mentioned in the discussion, but is briefly highlighted here in two and a half pages.

During the last six years we had the opportunity to use an already commercially available 3D ultrasound (US) image-guided radiotherapy (IGRT) device. We have put it through some tests and studied multiple applications among which are some new opportunities. This resulted in better understanding of the system, leading to some guidelines to prevent mistakes; a clinical workflow for the new application in the upper abdomen; and added value to the daily acquired images.

Besides the work in this thesis, another PhD thesis of Davide Fontanarosa on the speed of sound correction in US imaging was conducted in the same research group. The collaborations on that topic resulted in multiple papers and a correction method to diminish the aberrations due to differences in speed of sound in US imaging.

\section{CLINICAL AND SOCIETAL RELEVANCE}

\section{US image-guided radiotherapy: Where are we now and how did we contribute.}

Our research was useful for the radiotherapy community and for cancer patients.

We tested the robustness and made people aware of the critical issues in usage of this system. We proved the usability of the 3D US IGRT intramodality device and made it safer with some guidelines on the intermodaility registration stage.

Furthermore, we tested the system for use in the clinic with prostate and liver patients. Even though the results were positive, we did not implement it into the clinic due to practical reasons. (So our own patients did not benefit directly from this research yet.)

In current practise, prostate patient get four small metal markers inserted in their prostate. This is done with large needles transrectal or transperineal a week prior to the CT-imaging for patient planning, resulting in an increased infection risk and a week delay for the start of treatment.

We have compared the current practise with the use of the less invasive US procedure, and we have demonstrated US has comparable accuracy. The US workflow does require more practice, but it might be a desired alternative for patients who would like a less invasive procedure.

For liver patient, even more practice and experience is required, but the current practice is in need of improvements. Right now there are two main solutions to position the liver patients for their radiotherapy treatment. One of them is like with prostate, insertion of metal markers. But besides the complications already described for prostate, in liver you also have the risk of tumour spill, which means that there is a risk that you spread around tumour cells, which can result in new tumours around the track of the needle. Therefore, in our clinic, the current practise is the less invasive alternative without markers. In this case the patient will be positioned based on their bone positions, or if the cone 
beam CT image quality is good enough, based on soft tissue imaging around the liver. Unfortunately, the soft tissue contrast in the cone beam imaging devises currently in the treatment rooms is still relatively poor. This means that we cannot precisely/accurately visualize the tumour position. To compensate for this, wider safety margins are added to the target. With the US procedure, the imaging contrast is much better, leading to a more precise/accurate visualisation of the target, potentially resulting in smaller safety margins and better sparing of the healthy tissue.

For patients at our clinic this could have led to the introduction of a less invasive application of IGRT for prostate and a more precise/accurate treatment for liver cancer patients, but there is room for improvement in the US IGRT technique which might make it more attractive and hopefully will lead to clinical introduction in the future.

In the future, our clinic wants to get the patient more involved in the treatment decision, and I think that when prostate patients would be able to choose between US of metal markers, a lot will choose US. And I guess the same will be true for liver patient if they get the choice between US (even with all its peripheral equipment like active breathing control) or the current practice.

\section{Where do we want to go, and what did we do to make that happen}

For the small but growing number of US IGRT users our research provided a positive sound in the field that it deserves a (second) chance to prove its capabilities. Furthermore we managed to build a US IGRT platform/workshop to share and present ideas for current and future research. And the interest of a new major commercial partner who recently became interested in this topic was gained. With this new network we believe much more constructive partnerships and collaborations can be reached to make a move forward.

The radiotherapy field is more and more heading towards adaptive radiotherapy (ART) in which the radiation plan is adapted to the current anatomy of the day for each individual patient. With our research we showed that also US IGRT has capabilities that could be used for ART.

During the work with the system and the implementation of the technique, more and more topics and possible innovations became of interest for future research, but were not directly part of this thesis.

One example would be making the process less user dependent with more automation with for instance auto-contouring and non-handheld probes. Another application would be intrafraction motion monitoring, which helps us to image the irradiated structures, which would be a great step forward. Also improved contrast (e.g. contrast enhanced ultrasound) and functional imaging for targeting and treatment evaluation would benefit the field. So there is still a lot that can be done and our research group is continuing in helping to find the optimal solution.

\section{How will we get there}

The base and support for US IGRT is still rather small and US imaging is not seen as trendy. But innovating medical devices does not always require the development of new techniques. Sometimes the adoption and adaptation of old(er) techniques in new workflows is 
the innovation. Because US is an non-invasive technique maybe we should involve the patients more to convince the doctors. By making the patients aware of the different 'flavours' in the positioning techniques for radiotherapy treatment this might give them a preference for different treatment institutes. For instance through patient group websites this awareness could be spread.

But more importantly, with automated US probes, most of the US workflow issues that held back the usability so far will go away, and will no longer stand in the way of the benefits of a non-ionizing imaging technique. If we really are heading towards daily imaging before and possibly even during treatment, current imaging techniques increase the radiation burden on the healthy tissue. With the use of US IGRT, this damage to the healthy tissue can be avoided.

\section{ECONOMICAL RELEVANCE}

Because of the interest in tracking the target motion during treatment, there is also a need for phantoms to perform quality assurance and quality control. Our group has been working on a design for a 4D phantom that is currently under evaluation for patent submission together with a medical industry partner. 



\section{LIST OF PUBLICATIONS}





\section{PUBLICATIONS}

Published articles

A.J.E. Raaijmakers, B.W. Raaymakers, S. van der Meer, J.J.W. Lagendijk, "Integrating a MRI scanner with a $6 \mathrm{MV}$ radiotherapy accelerator: impact of the surface orientation on the entrance and exit dose due to the transverse magnetic field," Physics in Medicine and Biology 52 (4): 929-939 (2007).

A.C. Houweling, S. van der Meer, E. van der Wal, C.H.J. Terhaard, C.P.J. Raaijmakers, "Improved immobilization using an individual head support in head and neck cancer patients," Radiotherapy and Oncology 96 (1): 100-103 (2010).

D. Fontanarosa, S. van der Meer, E. Harris, F. Verhaegen, "A CT based correction method for speed of sound aberration for ultrasound based image guided radiotherapy," Medical Physics 38 (5): 2665-2673 (2011).

D. Fontanarosa, S. van der Meer, E. Bloemen-van Gurp, G. Stroian, F. Verhaegen, "Magnitude of speed of sound aberration corrections for ultrasound image guided radiotherapy for prostate and other anatomical sites," Medical physics 39 (8): 5286-5292 (2012).

D. Fontanarosa, S. van der Meer, F. Verhaegen, "On the significance of density-induced speed of sound variations on US-guided radiotherapy," Medical physics 39 (10): 6316-6323 (2012).

S. van der Meer, E. Bloemen-van Gurp, J. Hermans, R. Voncken, D. Heuvelmans, C. Gubbels, D. Fontanarosa, P. Visser, L. Lutgens, F. van Gils, F. Verhaegen, "Critical assessment of intramodality $3 D$ ultrasound imaging for prostate IGRT compared to fiducial markers," Medical Physics 40 (7): 071707 1-11 (2013).

E. Bloemen-van Gurp", S. van der Meer", J. Hendry, J. Buijsen, P. Visser, D. Fontanarosa, M. Lachaine, G. Lammering, F. Verhaegen, "Active Breathing Control in Combination With Ultrasound Imaging: A Feasibility Study of Image Guidance in Stereotactic Body Radiation Therapy of Liver Lesions," International Journal of Radiation Oncology* Biology* Physics 85 (4): 1096-1102 (2013).

D. Fontanarosa ${ }^{*}$, S. van der Meer", J. Bamber, E. Harris, T. O’Shea, F. Verhaegen, "Review of ultrasound image guidance in external beam radiotherapy: I. Treatment planning and inter-fraction motion management," Physics in Medicine and Biology 60 (3): R77-R114 (2015).

\footnotetext{
${ }^{*}$ Authors contributed equally
} 
S van der Meer, E Seravalli, D Fontanarosa, EJ Bloemen-van Gurp, F. Verhaegen, "Consequences of Intermodality Registration Errors for Intramodality 3D Ultrasound IGRT," Technology in Cancer Research \& Treatment June 5: 1-7 (2015).

Articles in submission

T. O'Shea, J. Bamber, D. Fontanarosa, S. van der Meer, F. Verhaegen, E. Harris, "Review of ultrasound image guidance in external beam radiotherapy part 2: Intra-fraction motion management and novel applications," Submitted to: Physics in Medicine and Biology

S. van der Meer ${ }^{*}$, S.M. Camps, , W. J.C. van Elmpt, M. Podesta, P. Gomes Sanches, B.G.L. Vanneste, D. Fontanarosa, F. Verhaegen, "Simulation of pseudo-CT images based on deformable image registration of ultrasound images: a proof of concept for transabdominal ultrasound imaging of the prostate during radiotherapy," Submitted to: Medical Physics

\section{Published abstracts}

A. Raaijmakers, B. Raaymakers, S. van der Meer, J. Lagendijk, "Monte Carlo simulations to quantify the dose distribution in a $1.5 \mathrm{~T}$ magnetic field for an integrated MRI-linear accelerator system: The impact of the ERE," ESTRO 25, Leipzig, Germany. Radiotherapy and oncology 81(Suppl 1): S84-S85 (2006).

S. van der Meer, A. Houweling, E. van der Wal, C. Terhaard, N. Raaijmakers, "Improved head and neck patient positioning using an individual head support," $10^{\text {th }}$ Biennial ESTRO Physics Meeting, Maastricht, The Netherlands. Radiotherapy and Oncology 92 (Suppl 1): S40 (2009).

N. Raaijmakers, S. van der Meer, A. Houweling, C. Terhaard, E. van der Wal, "Radiotherapy for CNS and H\&N cancer: the influence of the head support," $10^{\text {th }}$ Biennial ESTRO Physics Meeting, Maastricht, The Netherlands. Radiotherapy and Oncology 92 (Suppl 1): S61 (2009).

E. van der Wal, S. van der Meer, A.C. Houweling, C.H.J. Terhaard, C.P.J. Raaijmakers, "Improved fixation and position verification for salivary gland sparing head and neck IMRT," ASTRO's 51 ${ }^{\text {th }}$ Annual Meeting, Chicago, Illinois, USA. International Journal of Radiation Oncology* Biology* Physics 75 (Suppl 3): S586 (2009).

E. Bloemen- van Gurp, S. van der Meer, G. Lammering, F. Verhaegen, "3D ultrasound for IGRT in SBRT of liver lesions; why is EPI image guidance not sufficient?" ESTRO 29, Barcelona, Spain. Radiotherapy and Oncology 96 (Suppl 1): S419 (2010).

\footnotetext{
${ }^{*}$ Authors contributed equally
} 
S. van der Meer, E. Seravalli, E. Bloemen- van Gurp, F. Verhaegen, "Consequences of intermodality registration errors for an intramodality 3D ultrasound IGRT system," ESTRO 29, Barcelona, Spain. Radiotherapy and Oncology 96 (Suppl 1): S525 (2010).

D. Fontanarosa, S. van der Meer, F. Verhaegen, "Clinical evaluation of a speed of sound aberration correction algorithm in quantitative ultrasound-aided image guided radiother$a p y, " 11^{\text {th }}$ Biennial ESTRO Physics Meeting, London, UK. Radiotherapy and Oncology 99 (Suppl 1): S215 (2011).

E. Bloemen-van Gurp, S. van der Meer, P. Visser, J. Buijsen, I. Steenbakkers, G. Lammering, F. Verhaegen, "A new solution for IGRT in SBRT of liver lesions, based on 3D us imaging combined with active breathing control," $11^{\text {th }}$ Biennial ESTRO Physics Meeting, London, UK. Radiotherapy and Oncology 99 (Suppl 1): S450-S451 (2011).

S. van der Meer, E. Bloemen-van Gurp, J. Hermans, D. Heuvelmans, R. Voncken, C. Grubbels, M. Berbée, E. Seravalli, P. Visser, L. Lutgens, F. van Gils, F. Verhaegen, "Prostate image guided radiotherapy using quantitative $3 D$ ultrasound imaging: inter-and intraoperator aspects," $11^{\text {th }}$ Biennial ESTRO Physics Meeting, London, UK. Radiotherapy and Oncology 99 (Suppl 1): S468 (2011).

E. Bloemen, S. van der Meer, P. Visser, J. Buijsen, I. Steenbakkers, G. Lammering, F. Verhaegen, "3D US imaging combined with active breathing control as a new solution for IGRT in SBRT of liver lesions," 2011 Joint AAPM/COMP Meeting, Vancouver, Canada. Medical Physics 38 (6): 3390 (2011).

D. Fontanarosa, S. van der Meer, E. Harris, F. Verhaegen, "Speed of sound aberration correction in quantitative ultrasound-aided image guided radiotherapy," 2011 Joint AAPM/COMP Meeting, Vancouver, Canada. Medical Physics 38 (6): 3747 (2011).

S. van der Meer, E. Bloemen, J. Hermans, R. Voncken, D. Heuvelmans, C. Gubbels, M. Berbee, E. Seravalli, P. Visser, L. Lutgens, F. van Gils, F. Verhaegen, "Operator uncertainties in a soft tissue 3D ultrasound image guided radiotherapy system," 2011 Joint AAPM/COMP Meeting, Vancouver, Canada. Medical Physics 38 (6): 3813 (2011).

G. Lammering, E. Bloemen- van Gurp, S. van der Meer, J. Buijsen, P. Visser, F. Verhaegen, "Active breathing control during 3D ultrasound image guidance for SBRT of liver lesions," ASTRO Annual Meeting, Miami Beach, Florida, USA. International Journal of Radiation Oncology* Biology* Physics 81 (Suppl 2): S351-S352 (2011).

S. van der Meer, E. Bloemen-van Gurp, D. Fontanarosa, P. Visser, L. Lutgens, F. van Gils, F. Verhaegen, "Prostate IGRT using quantitative 3D ultrasound imaging: intramodality US vs. MV imaging of implanted markers," ESTRO 31, Barcelona, Spain. Radiotherapy and Oncology 103 (Suppl 1): S135 (2012). 
F. Verhaegen, S. van der Meer, D. Fontanarosa, "On the significance of density-induced speed of sound variations on ultrasound-guided radiotherapy," ESTRO 31, Barcelona, Spain. Radiotherapy and Oncology 103 (Suppl 1): S340 (2012).

S. van der Meer, J. Wong, E. Bloemen, D. Fontanarosa, M. Lachaine, F. Verhaegen, "Towards real-time ultrasound image-guided abdominal radiotherapy," AAPM $54^{\text {th }}$ Annual Meeting, Charlotte, NC, USA. Medical Physics 39 (6): 3934 (2012).

D. Fontanarosa, S. van der Meer, E. Bloemen, F. Verhaegen, "On the significance of density-induced speed of sound variations on ultrasound-guided radiotherapy," AAPM $54^{\text {th }}$ Annual Meeting, Charlotte, North Carolina, USA. Medical Physics 39 (6): 4018 (2012).

S. van der Meer, D. Bouvy, B. Reniers, D. Fontanarosa, F. Verhaegen, "Ultrasound IGRT: Deformable image registration of daily ultrasound images to derive daily CT images," $2^{\text {nd }}$ ESTRO Forum, Geneva, Switserland. Radiotherapy and Oncology 106 (Suppl 2): S330-S331 (2013).

S. van der Meer, D. Bouvy, B. Reniers, D. Fontanarosa, F. Verhaegen, "Ultrasound based deformable image registration: daily CT images derived from daily IGRT ultrasound," AAPM $55^{\text {th }}$ Annual Meeting, Indianapolis, Indiana, USA. Medical Physics 40 (6): 375 (2013).

S. van der Meer, N. Menten, L. Verheesen, J.J. Jager, J.D. Zindler, D.B.P. Eekers, G. Bosmans, L.H.P. Murrer, "Evaluation of cranial setup accuracy: a double shell positioning system versus an in-house 3-point mask solution," $3^{\text {rd }}$ ESTRO Forum, Barcelona, Spain. Radiotherapy and Oncology 115 (Suppl 1): S819-S820 (2015).

Workshop presentations

S. van der Meer, D. Bouvy, B. Reniers, D. Fontanarosa, F. Verhaegen, "deformable image registration in ultrasound IGRT: daily CT images derived from ultrasound," $1^{\text {st }}$ Ultrasound Guided Radiotherapy Workshop, Maastricht, The Netherlands (2013).

S. van der Meer, D. Fontanarosa, J. Bamber, E. Harris, T. O'Shea, F. Verhaegen, "The use of ultrasound image guidance in external beam radiotherapy for treatment planning and inter-fraction motion management, a review, " $2^{\text {nd }}$ Ultrasound Guided Radiotherapy Workshop, Cookham, UK (2015).

S. Camps, S. van der Meer, D. Fontanarosa, F. Verhaegen, "Pseudo-CT scan creation based on ultrasound to ultrasound deformable image registration in prostate cancer patients for radiotherapy treatment adaptation," $2^{\text {nd }}$ Ultrasound Guided Radiotherapy Workshop, Cookham, UK (2015). 
T. O'Shea, J. Bamber, S. van der Meer, D. Fontanarosa, F. Verhaegen, E. Harris, "The use of ultrasound image guidance in external beam radiotherapy for intra-fraction motion management, a review," $2^{\text {nd }}$ Ultrasound Guided Radiotherapy Workshop, Cookham, UK (2015).

P. Gomes Sanches, S. van der Meer, E. Stevens, L.M.M. Huiberts, D. Fontanarosa, A. Lataster, F. Verhaegen, "Deformable $4 D$ ultrasound phantoms in radiation therapy," $2^{\text {nd }}$ Ultrasound Guided Radiotherapy Workshop, Cookham, UK (2015). 

DANKWOORD 



\section{DANKWOORD}

Ik ben veel mensen dankbaar voor hun directe en indirecte bijdrage aan mijn werk, want dit proefschrift was nooit in deze vorm tot stand gekomen zonder de hulp van heel veel mensen.

Om te beginnen wil ik GROW (School for Oncology and Developmental Biology, Maastricht University) bedanken voor hun financiering en de algemene begeleiding.

Maar in het bijzonder wil ik natuurlijk prof. dr. Ir. Verhaegen bedanken. Frank bedankt dat je me hebt uitgenodigd voor een gesprek voor een vacature die ik volledig gemist had en waar ik pas na de reactietermijn indirect op reageerde. Ik ben blij dat ik de kans heb gekregen om op dit klinische project veel praktische en wetenschappelijke ervaring op te doen. Je bent zeer betrokken bij de projecten van al je studenten en ik heb veel van je geleerd. Maar ik ben je natuurlijk met name zeer dankbaar voor je betrokkenheid en geduld in mijn project. Het liep niet altijd even soepel, maar door jouw focus ben ik op de goede weg gebleven en hebben we nu eindelijk deze finish gehaald.

Dr. Lutgens, Ludy, bedankt voor het bijspringen als copromotor en je betrokkenheid in het project.

Furthermore I would like to thank the members of my assessment committee, prof. dr. ir. W.H. Backes, Dr. M.E. Kooi, prof. dr. ir. J.J.W. Lagendijk and prof. dr. med. F. Lohr for their time and effort in appraising this thesis on short notice.

Esther, jij hebt me op weg geholpen in de start van het project. Door jouw begeleiding kon ik snel het prostaatproject van je overnemen en was ik je back-up voor het leverproject. Ik vind het jammer dat je uiteindelijk naar een ander project bent overgestapt, maar ben je heel erg dankbaar voor de samenwerking en betrokkenheid ook later toen je niet meer deel uit maakte van de US-projectgroep. Ik ben dan ook heel blij dat je tijdens mijn verdediging naast en achter me wilt staan als paranimf.

Voor de klinische studies ben ik natuurlijk de patiënten erg dankbaar voor hun medewerking maar ook de artsen en AIOS die hen gevraagd hebben om mee te werken aan onze studies. Daarnaast waren Francis, Ludy, Jeroen, Guido en Ben ook betrokken bij de opzet van de studies, waarvoor dank.

Robert, Jolanda, Denise, Carol, Debbie en Marlies, bedankt voor jullie tijd en toewijding aan de prostaat- en leverstudie. Samen met de andere laboranten die getraind waren voor US hebben jullie het mogelijk gemaakt dat we patiëntenscans konden verzamelen voor de studies. 
Bianca en Liesbeth, bedankt voor jullie poging om ook voor borst een studie op te zetten. Helaas zagen we niet genoeg met de US waardoor het niet tot een werkelijke patiëntenstudie heeft geleid.

Bianca, bedankt dat je me later nog geholpen hebt met het verschuiven van de bundels bij de leverdataset.

Liesbeth, bedankt voor je interesse in het project en de tijd die je er in hebt gestoken, nu ook als lid van de promotiecommissie.

Voor het logistiek mogelijk maken van de patiëntenstudies ben ik zowel Rene Hermans, Mia Brouns als de 'Patiënten planners' erg dankbaar. Jullie waren niet altijd blij om mij te zien, want dat betekende namelijk dat ik weer het schema in de war wilde schoppen. Ondanks dat hebben jullie me altijd vriendelijk geholpen.

Ann, Ruud en de rest van datamanagement wil ik graag bedanken voor hun gezelschap in het begin en natuurlijk voor hun administratieve ondersteuning en hulp bij de statistiek.

Remco, John en Shane, bedankt voor jullie bereidwilligheid om als proefpersoon op te treden. Shane, is this where it all started?

Naast de patiëntenstudies was er het 'error propagation' werk met Enrica, en de 'speed of sound' met Davide.

Enrica, bedankt voor je samenwerking met de fantoomscans maar natuurlijk ook voor je gezelschap buiten het werk in het zwembad.

Davide, thanks for your collaboration and encouragements that I needed from time to time. And thank you for the effort you are taking in assessing my work as a corona member.

Hans en Chris, bedankt voor de tools en onderdelen voor de fantomen die jullie voor ons gemaakt hebben.

De KFMers, en in het bijzonder Peter Roman, bedankt voor jullie ondersteuning met de US apparatuur.

Peter Visser, jij kreeg als klinisch fysicus de klinische introductie van het US systeem onder je hoede en daarmee raakte je ook betrokken bij mijn onderzoek. We zijn een heel eind gekomen en waren er bijna toen de stekker er definitief uit ging. Bedankt voor je toewijding tijdens het proces. De kliniek heeft US uiteindelijk niet geïntroduceerd, maar ik heb veel geleerd over wat er allemaal bij komt kijken om een nieuwe techniek te introduceren. Onder andere het maken van een SAFER-analyse waarvoor ik ook Petra wil bedanken voor de begeleiding.

I would also like to thank the people from Resonant Medical, now Elekta. Janet, Kevin, Martin, Rui, David, Tony, Sergei, Sebastien, Denis and Philippe, you were there for me when I needed advise and/or help on practical issues like delineations, workflows, system settings, restoring of the database/server and much more, but you were also involved in the broader scoop of the project itself. Thank you all for your dedication. 
Ook de firma's Bracco en Nano4lmaging worden bedankt voor hun betrokkenheid in de zoektocht naar verbeteringen in de zichtbaarheid van levertumoren en borstmarkers.

ICT (Rob, Nigel, Antoine, Pim, Joost, en ik ben er vast een paar vergeten ...), bedankt voor het steeds weer openzetten van de internetconnecties voor WebEx-sessies met Canada. Maar natuurlijk ook bedankt voor het instellen van een back-up oplossing nadat alle data bijna verloren was gegaan nadat de US-server stuk ging. Ik heb regelmatig met verschillende problemen aan jullie bureau gestaan, en gelukkig was meestal toch een individuele oplossing voor mij te vinden. Bedankt voor jullie flexibiliteit in dit project.

Sonia, Ellen en Rianne, bedankt voor jullie administratieve ondersteuning.

Brigitte, thank you for your help with the deformation study. In the end we chose another program to perform the deformations, but due to your help in the beginning we did not have to start from scratch.

Saskia, Damien, Saskia en Ester, ik ben bij geen van jullie de (officiële) begeleider geweest, maar vond het wel leuk om met jullie samen te kunnen werken. Saskia Camps, onze samenwerking ging verder op het werk dat in samenwerking met Damien was gedaan en jij hebt er voor gezorgd dat de workflow van het toepassen van de deformaties een stuk makkelijker werd en je hebt een stap gezet in de optimalisatie van de parameters.

Emma, Jeff and Tuathan, it has been a pleasure to work with you on the review and the workshops. I'm looking forward to the publication of the second part of the review. US radiotherapy will gain a lot with the knowledge of your group.

Pedro, it was great to work with you on the new project. Even though it was from the sideline, I like that you and Davide kept me involved.

Wouter, het was altijd fijn om met je te kunnen brainstormen over vragen en problemen. Ook al was je niet direct betrokken bij mijn project, je deur stond en staat altijd open en je hebt me vaak weer op weg geholpen.

Mark, besides the people from downstairs you were my personal ICT guy. You not only helped me with a lot of practical issues, but were also always available for brainstorm sessions about work and life. I still owe you a lot of drinks.

My fellow researchers Hugo, Steven, Katia, Merel, Marco, Ruud, Georgi, Georgy, Daniela, Francesco, Hoda, Guillaume, Emmanuel, Patrick, Celine, Pouya, Mathieu, Scott, Raghu, Shane, Sanaz, Lucas, Daan, Adriana, Janita, Sean, Evelyn, Aniek, Ralph, Sara, Karen, Ruben, Timo, Jurgen, Gabriel, Stefan, Jonathan, Mark, Lotte, Isabel, Nicolle, Maaike and Patricia, I would like to thank you for all the social, work and non-work related activities. Sometimes your company was enough to make me come to the office. I'm really going to miss all the nice and sometimes really weird conversations over lunch and/or coffee. 
Aniek, bedankt voor je nuchtere houding en het herkenbare enthousiasme voor wandelen en het buitenleven. Ik ben blij dat je samen met Esther mijn paranimf wilt zijn.

De klinisch fysici, Lars, Aswin, Mirko, Geert, Bas, Michel, Ans, Michiel, Fiere, Chin Loon, Andre en Erik wil ik bedanken voor hun samenwerking op onderzoeksgebied maar ook voor hun samenwerking toen ik een jaar als project fysicus werkte. Bedankt dat ik een kijkje in jullie keuken mocht nemen en ervaring op heb kunnen doen.

Niet alleen de mensen van Maastricht hebben me op weg geholpen, maar voor mijn promotie was de groep in Utrecht daar al mee gestart. Jan, Bas, Niels, Annette, Edwin, Alexander, Ellen, Mette, Sjoerd, Joana, Michiel en de rest, bedankt voor jullie enthousiasme voor het vakgebied en het mij op weg helpen met netwerken tijdens de ESTRO van 2009 in Maastricht. Greetje bedankt voor het voorstellen aan Steven, en Steven bedankt voor de rondleiding bij Maastro en het in contact brengen met Frank.

Hoda, Ali, Sara, Nuno, Karen, Philip and Ruud you have become great friends. Thank you all for all the nice moments we shared outside of Maastro.

Lotte, Jefrey, Jeroen, Marja, Roel en Jaimi, het contact werd misschien wat minder frequent nadat wij en/of jullie verhuisd zijn. Maar ik ben blij dat we elkaar nog regelmatig zien of spreken.

Jeroen, ook nog bedankt voor het aanleveren van artikelen waarvoor de UM geen toegang had.

Hieke, Selma, Maarten, Jan Jitse, Jeroen, bedankt voor jullie goede voorbeeld en gezelschap.

Peter, Judith, Åsa, Marco, Allert, Miranda, Roselinde, Jasmijn, Mees en Pepijn, in de weekenden hebben jullie regelmatig voor de welkome afleiding gezorgd. Daarnaast ook bedankt voor jullie steun en interesse.

Mam, het is raar om je te bedanken voor de aanleiding van mijn enthousiasme en motivatie voor het vakgebied radiotherapie. Toch heeft het me richting in het leven gegeven, waarvoor dank.

Pap en Mam, jullie hebben altijd voor mij klaar gestaan en onvoorwaardelijk gesteund. Zelfs ons huis werd door jullie verzorgd en mankementen gemaakt als wij met vakantie waren. Bedankt voor jullie lieve en goede zorgen.

Geert, waar moet ik beginnen. Je bent met me mee verhuisd naar het zuiden en bent altijd mijn steun en toeverlaat geweest. Ik heb het je zeker niet gemakkelijk gemaakt en tegenslagen op het werk kwamen de sfeer thuis niet altijd ten goede. Maar ik ben blij dat je er altijd voor me was, ook wanneer jij zelf eigenlijk te druk was met je werk. Nu mijn promotie af is kunnen we eindelijk verder met het maken van plannen voor de toekomst die al snel dicht bij komt. Maar, zolang die toekomst met jou samen is komt het vast goed. 
Louis/Lars/IJsbrand/...., zo klein, maar groot genoeg voor dat extra beetje motivatie om het nu snel af te ronden. Bedankt voor de deadline.

If I have forgotten to mention you, please don't take it personal. Your contribution to my work and life was and is highly appreciated, just due to stress, hormones and time pressure I forgot to add you to this list. 




\section{CURRICULUM VITAE}

Skadi van der Meer werd geboren op 11 maart 1981 te Zevenaar. In 1998 behaalde zij het Havo diploma om vervolgens in 2000 het VWO te voltooien, beide op het Liemers College te Zevenaar. Na het behalen van het VWO diploma is ze aan de Universiteit Utrecht Natuurkunde gaan studeren. Al in het eerste jaar kwam ze in aanraking met klinische fysica en was daar zo door geboeid, dat ze in 2006 haar bachelor besloot af te ronden met een afstudeerproject bij de radiotherapie afdeling van het Universitair Medisch Centrum Utrecht. Om in de richting van klinische fysica te blijven is ze na het afronden van de bachelor Natuurkunde in 2006, de master Biomedical Image Science gaan volgen, wederom aan de Universiteit Utrecht. Ook nu werd het afstudeerproject bij de radiotherapie afdeling van het Universitair Medisch Centrum Utrecht gedaan, en ronde zij hier in 2009 haar masteropleiding me af. $\mathrm{Na}$ een korte tijd te hebben gezocht naar een geschikte promotieplaats, vond zij deze snel bij Prof. F. Verhaegen van de MAASTRO clinic in Maastricht en startte zij 1 december 2009 met haar promotieonderzoek binnen de vakgroep radiotherapie van de Universiteit Maastricht. Op 1 december 2013 heeft zij een jaar als project fysicus gewerkt binnen de Klinische Fysica Groep van MAASTRO clinic, en per 1 augustus 2015 is ze voor 4 maanden aangesteld als onderzoeker voor een project bij de research groep van Prof. P. Lambin. 